\title{
The Genetic and Physiological Characteristics of the \\ Symbiodinium spp. in the endemic anemone
}

\section{Anthopleura aureoradiata}

\author{
Jennifer N Howe
}

\author{
A thesis \\ submitted to Victoria University of Wellington \\ in partial fulfilment of the requirements for the degree of \\ Masters of Science in Marine Biology
}

2013

VICTORIA UNIVERSITY OF WELLINGTON

Te Whare Wānanga o te Ūpoko o te Ika a Māui

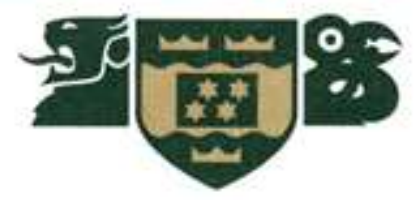




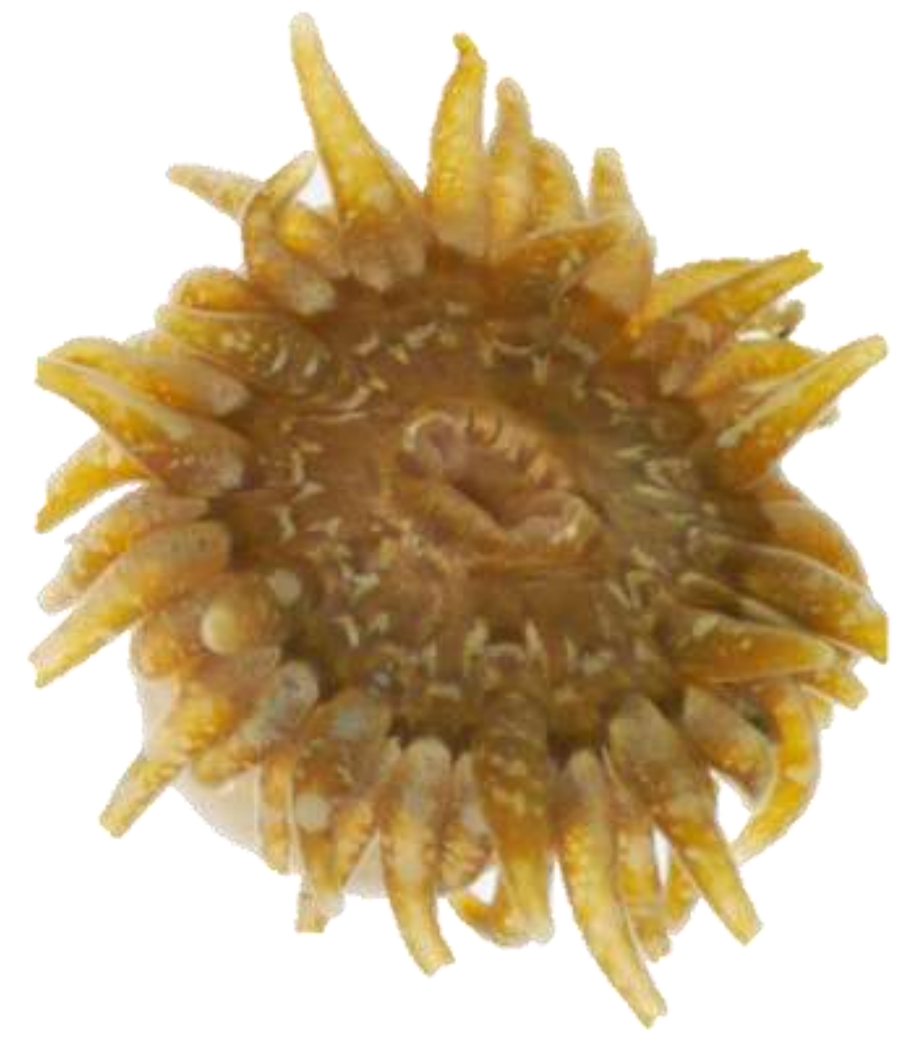

Anthopleura aureoradiata 


\begin{abstract}
Photosynthetic dinoflagellates of the genus Symbiodinium form symbiotic relationships with many marine hosts, including cnidarian corals and sea anemones. This partnership is extremely successful in tropical waters leading to a great diversity of coral species and Symbiodinium types. Environmental condition in the tropics are stable, changes to which can lead to destabilization of the symbiotic interactions between the host and symbiont, which in turn can lead to total breakdown of the partnership and expulsion of the symbiont. Temperate symbiotic cnidarian species, especially sea anemones, are less common but locally abundant. Environmental conditions are highly variable with extreme differences in light and temperature. Adaptation to these conditions has led to the success of resilient partnerships, but also to less diversity of Symbiodinium types.
\end{abstract}

This study looked at the relationship between the endemic New Zealand anemone, Anthopleura aureoradiata, and its symbiotic relationship with the Symbiodinium cells it harbours. The aim was to determine why and how this symbiotic relationship is so resilient to the temperate conditions by 1) determining the molecular identity of the Symbiodinium spp. within the anemone, throughout its latitudinal range and through the seasons, and whether any seasonal changes differed between two habitats, the rocky shore and mudflats; 2) comparing the identity of the Symbiodinium spp. in New Zealand with those from four species of anemones from Europe (Cereus pedunculatus, Anthopleura ballii and Anemonia viridis from the south-west of England and Aiptasia mutabilis from Brittany (France)) to establish any differences or similarities between the northern and southern hemispheres; 3) determining whether resilience to environmental conditions is attributed to the Symbiodinium photoprotective mechanisms.

A. aureoradiata were collected in early autumn in five sites from the top (Parengarenga Harbour) to the bottom (Stewart Island) of New Zealand for the latitudinal study. Seasonal anemones were collected from a rocky shore in Wellington Harbour (Point Halswell, Kau Bay) and a mudflat at Pauatahanui Inlet. Symbiodinium types were identified to subcladal level using ITS2 sequencing. A low diversity of types was found, with all anemones harbouring algal cells identified as being similar, or identical to, Symbiodinium sp. Mediterranean clade A (Med clade A) and Symbiodinium sp. Amed 
(Amed). 96.55\% of the anemones from the latitudinal study, all the winter anemones, $87.50 \%$ of the summer anemones and almost $78 \%$ of the autumn anemones harboured Symbiodinium cells most similar or identical to Med clade A. All Symbiodinium sequences from the European anemones also were identified as being similar or identical to Med clade A or Amed, suggesting that the Symbiodinium in A. aureoradiata are likely not endemic. It is not known whether anemones harbour both types simultaneously and whether a change in dominant symbiont type occurs with seasons within anemones by "shuffling".

The photophysiology of the Symbiodinium cells isolated from the anemones was studied using an Imaging-PAM fluorometer whilst being maintained in six light and temperature treatments. The photosynthetic rate of PSII, energy quenching by NPQ, and photosystem recovery were measured to determine whether the Symbiodinium cells had a strong capacity for photoprotection and were able to down-regulate quickly to reduce photodamage to the chloroplast. The main outcome of this study is that the Symbiodinium cells within A. aureoradiata are very effective in protecting themselves against photo-damage by activating an efficient NPQ system. Down-regulation of the quantum efficiency of PSII under high light conditions appeared to cease altogether. Whether this was a true measurement of down-regulation to stop photodamage, or whether these clade A types use an alternative electron transport that bypasses PSII, and can therefore not be measured with the I-PAM fluorometer technique used, needs to be addressed in future studies. 


\section{Declaration}

\section{STATEMENT OF ORIGINALITY}

The following work presented in this thesis is, to the best of my knowledge, original. It is a representation of my own work throughout my Masters of Science course, except as acknowledged throughout the text. All figures presented represent original work unless otherwise stated. The contained material has not been submitted for any degree at this or any other institution. 


\section{Acknowledgements}

There are many people I would like to thank who helped me with my work. First on the long list are my friends and colleagues from the Davy Lab. For taking me on and helping greatly with the production of this thesis, I thank Dr Simon Davy. For getting me started on the molecular work, I'm extremely grateful to Dr Paul Fisher. Thanks to Steffi Pontasch, for giving me so much of her time to answer so many of my questions, and always with a smile. Thanks to Dr Suzanne Becker, Thomas Krueger and Shaun Wilkinson for clarifying the mysteries of the I-PAM, 'trees' and statistics. Thanks to all my anemone collecting buddies from the lab, especially Emma Gibbin, who travelled up the North Island with me, and Steffi, who spent many hours crawling over the rocks at Point Halswell. Special thanks though to the most intrepid of the anemone hunters, Anne Wietheger, who travelled south with me to Stewart Island, and who also could be relied upon to face the dawn on my many, early morning collection trips. Fun times!

Thanks to Dr Ross Hill, The University of New South Wales, who gave me advice on how to use DCMU on my anemones and to Dr Xavier Pochon, Cawthron Institute, for looking through my sequences and advising me on the structure of the genetic trees to use. Thanks to the technicians in the School of Biological Sciences, especially Neville Higgison, who rewired a room and helped in supplying much of the electrical equipment needed. I would not have been able to make a comparison of the New Zealand Symbiodinium types with the European ones without the assistance of Dr Keith Hiscock from the Marine Biological Association in Plymouth, England, who collected all the English anemones, and Laurent Leveque and Sylvie Tanguy from the Station Biologique de Roscoff, Brittany.

Financial assistance was given to me by Victoria University with the Graduate Scholarship and the Masters (by Thesis) Scholarship, for which I am exceedingly grateful. Also thanks to the grandparents of Alison Morton, for the scholarship they have set-up in her memory, and to the Wellington Branch, New Zealand Federation of Graduate Women for the Masters by Thesis Scholarship awarded to me. 
My biggest thanks has to go to my family, Chris, Liam and Adam. My boys have put up with having an absent mother for too long. Without Chris this long slog would have been tremendously difficult. Life was made a lot easier with his support. Thank you; it's time to show the three of you my appreciation.

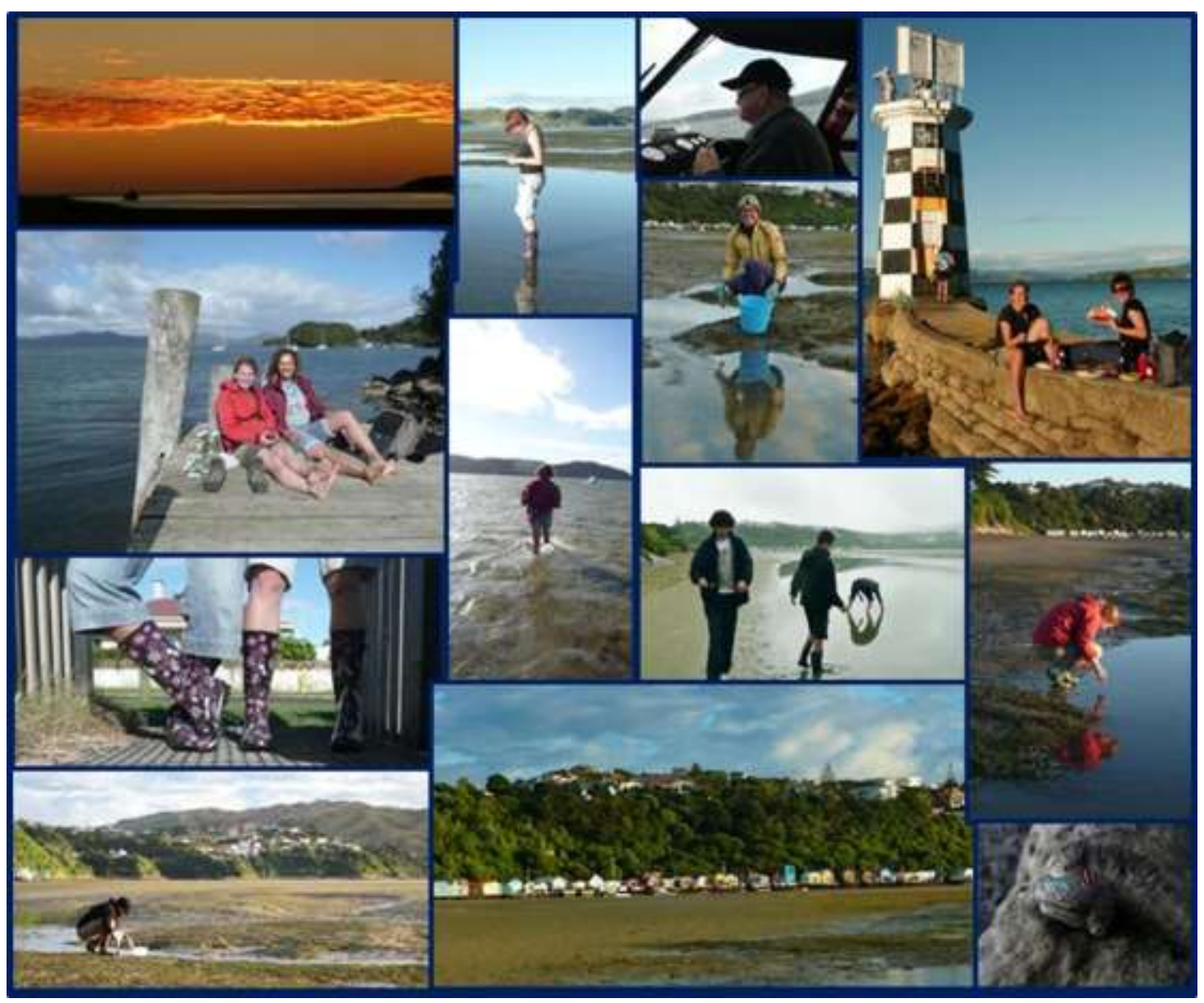




\section{$\underline{\text { Table of Contents }}$}

ABSTRACT

DECLARATION V V

ACKNOWLEDGEMENTS VI

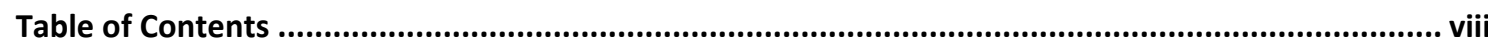

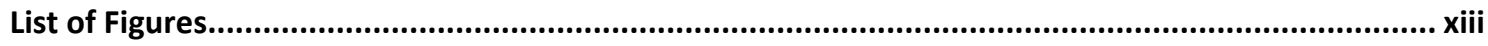

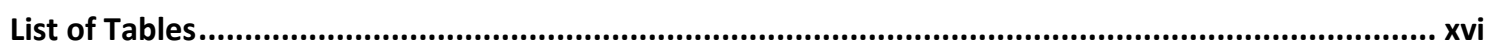

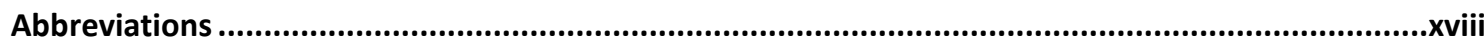

CHAPTER 1 - INTRODUCTION 1

THE CNIDARIAN-DINOFLAGELLATE SYMBIOSIS 1

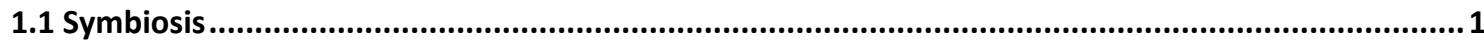

$\begin{array}{ll}\text { 1.1.1 Definition and types of symbiosis } & 1\end{array}$

1.1.2 Cnidarian-dinoflagellate symbiosis 3

1.2 Cellular basis of cnidarian-dinoflagellate symbiosis .........................................................6

1.2.1 Establishment of the symbiosis 6

$\begin{array}{ll}\text { 1.2.2 Maintenance of the symbiosis } & 7\end{array}$

1.2.3 Breakdown of the symbiotic partnership $\quad 8$

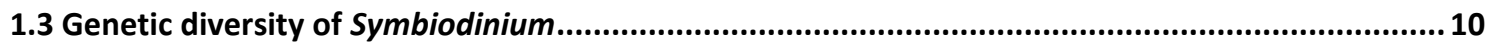

$\begin{array}{ll}\text { 1.3.1 Molecular identification within the genus Symbiodinium } & 10\end{array}$

$\begin{array}{ll}\text { 1.3.2 Biogeographic and ecological variability in diversity } & 13\end{array}$

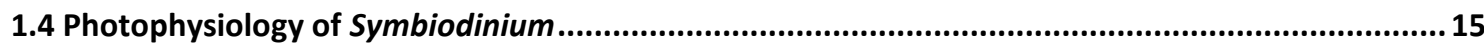

1.5 Tolerance and acclimation to environmental stress by change of symbiont ...............................21

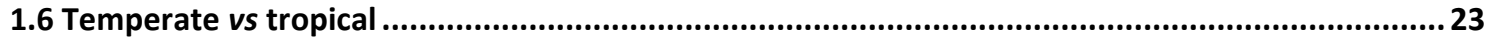

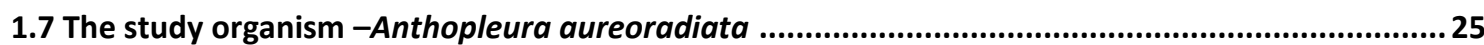


CHAPTER 2

THE BIOGEOGRAPHY OF SYMBIODINIUM SPP. WITHIN THE NEW ZEALAND

ENDEMIC ANEMONE, ANTHOPLEURA AUREORADIATA

2.1 Introduction .29

2.1.1 Diversity within the genus Symbiodinium

CHAPTER 3

PHOTOPHYSIOLOGY OF ISOLATED SYMBIODINIUM CELLS FROM THE 
$\begin{array}{ll}\text { 3.1.3 Photoprotection } & 66\end{array}$

$\begin{array}{ll}\text { 3.1.4 Temperate resilience } & 68\end{array}$

$\begin{array}{ll}\text { 3.1.5 Aim of the study } & 69\end{array}$

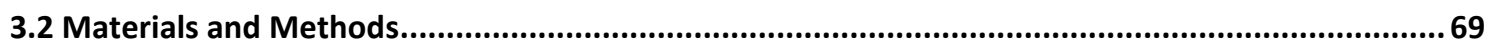

$\begin{array}{lc}3.2 .1 \text { Sampling } & 69\end{array}$

$\begin{array}{ll}\text { 3.2.2 Experimental set-up } & 70\end{array}$

$\begin{array}{ll}\text { 3.2.3 Experimental procedure } & 71\end{array}$

$\begin{array}{ll}\text { 3.2.4 Cell counts } & 73\end{array}$

3.2.5 Photophysiological measurements $\quad 73$

$\begin{array}{lr}\text { 3.2.6 Statistical analysis } & 76\end{array}$

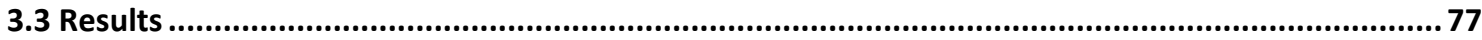

$\begin{array}{ll}\text { 3.3.1 Algal cell density } & 77\end{array}$

$\begin{array}{ll}\text { 3.3.2 Fluorescence parameter measurements } & 77\end{array}$

3.3.2 1 Maximum quantum yield $\left(F_{v} / F_{m}\right) \quad 77$

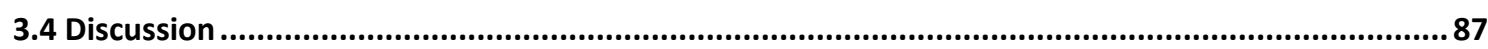

$\begin{array}{ll}\text { 3.4.1 Down-regulation } & 87\end{array}$

$\begin{array}{ll}\text { 3.4.2 Photosynthetic efficiency } & 91\end{array}$

CHAPTER 4

GENERAL DISCUSSION

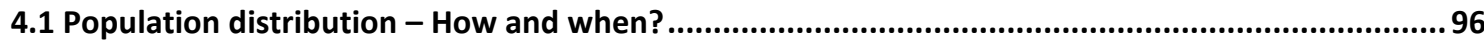

$\begin{array}{ll}4.1 .1 \text { Ancient Origins } & 96\end{array}$

$\begin{array}{ll}\text { 4.1.2 Vectors of connectivity } & 98\end{array}$

4.2 What is special about clade $A$ as a temperate symbiont? ...................................................... 101 
APPENDIX 1 - CLIMATIC CONDITIONS IN NEW ZEALAND FOR 5 YEARS PRIOR

APPENDIX 2 - MOLECULAR SEQUENCES OF SYMBIODINIUM TYPES IN THIS STUDY

A2.1 Coding used to identify samples in this study

\section{APPENDIX 4 - TIME TRIALS TO DETERMINE HOW LONG ISOLATED} SYMBIODINIUM CELLS REMAINED PHOTOSYNTHETICALLY HEALTHY 
A6.4: Mean and $S E$ of $F_{0}, F_{m}$ and $F_{t}$ 149

A6.5: Statistical results for rETR $_{\max }$ 151

A6.6: Statistical Results for $E_{k}$ .153

A6.7: Statistical Results for $\mathrm{NPQ}_{\max }$ .156

REFERENCES 


\section{List of Figures}

\section{Chapter 1}

Figure 1.1: Forms of endosymbiosis ................................................................

Figure 1.2: Cnidarians include corals, jellyfish and sea anemones..............................

Figure 1.3: The study organism Anthopleura aureoradiata, with its associated Symbiodinium spp.

Figure 1.4: The arrangement of algal cellular structures, algal plasma membrane and cell wall in the cnidarian-Symbiodinium symbiosis, all surrounded by the host-derived membrane 6

Figure 1.5: The five mechanisms of cellular breakdown

Figure 1.6: Schematic of nuclear and organelle genomes targeted for molecular differentiation of the genus Symbiodinium 10

Figure 1.7: Proposed ITS2 secondary structure for Symbiodinium type A1 11

Figure 1.8: The phylogenetic relationships between the major clades of Symbiodinium.

Figure 1.9: Three proposed impacts of elevated temperature and light on the photosystem of Symbiodinium cells 16

Figure 1.10: "Switching" and "shuffling"of Symbiodinium types 22

Figure 1.11: Anthopleura aureoradiata in its habitat. 26

Figure 2.1: Representation of a Symbiodinium secondary structure with five helices... 31

Figure 2.2: The two variations of secondary structures of Symbiodinium ITS2 and its relationship with the cladal phylogeny. 32

Figure 2.3: Anthopleura aureoradiata. 35 
Figure 2.4: Collection sites around New Zealand for the latitudinal molecular study. . 37

Figure 2.5: Collection sites and habitats around Wellington. .39

Figure 2.6: Photographs of stained SSCP gels 48

Figure 2.7: ITS2 sequence of D5 and its secondary fold 51

Figure 2.8: Secondary structures of ITS2 sequences 52

Figure 2.9: Maximum-likelihood tree of sampled Symbiodinium types. .54

Figure 2.10: Bayesian tree of sampled Symbiodinium types. 55

Figure 2.11: Diagrammatic representation of the different levels of phylogenetic resolution identified by the molecules analysed in Symbiodinium cells. 62

Figure 3.1: Monthly temperature and solar irradiance (mean \pm SE) near Pauatahanui Inlet .70

Figure 3.2: Algal pellet after centrifuging .71

Figure 3.3: Set-up of the I-PAM and the 15 vials measured for each light treatment ... 73

Figure 3.4: Imaging-PAM and computer set-up 73

Figure 3.5: A typical photosynthesis versus irradiance (P-I) curve............................ 76

Figure 3.6: Cell density at the end of the experiment .77

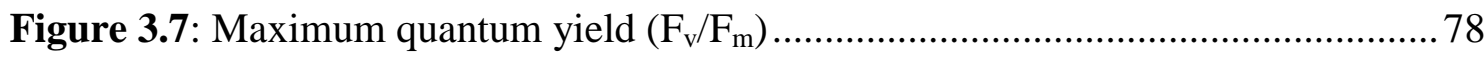

Figure 3.8: Fluorescence parameters through dark-induction measurements............... 81

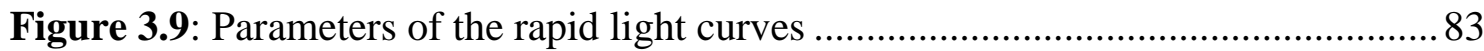

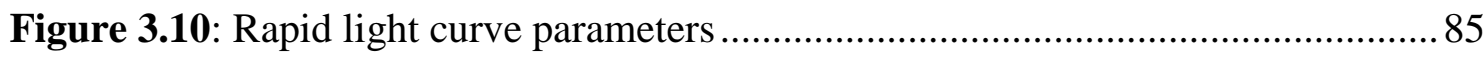

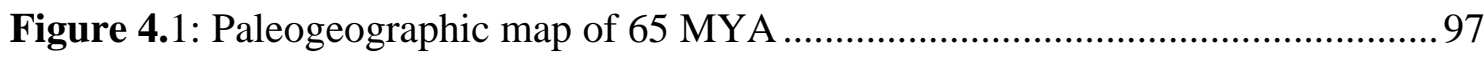

Figure 4.2: A) Cotylorhiza tuberculata, the Mediterranean fried egg jellyfish and Phyllorhiza punctate, the Australian spotted jellyfish 100 
Figure A1.1.1: Mean ( \pm SE) daily temperature and radiance through the seasons for 5 years prior to the molecular latitudinal study

Figure A1.2.1: Mean ( \pm SE) daily temperature and radiance through the seasons for 5 years prior to the molecular seasonal and habitat study 109

Figure A2.4.1: ITS2 secondary structures for Symbiodinium clade A as proposed by Hunter et al (2007).

Figure A3.1.1: Chlorophyll fluorescence induction kinetics using DCMU

Figure A3.2.1: $\mathrm{RLC}$ testing $\mathrm{MgCl}$ concentrations

Figure A3.2.3: Chlorophyll fluorescence induction kinetics of the DCMU concentration trials on isolated Symbiodinium cells

Figure A3.2.4: Acetone dilution tests. 136

Figure A3.2.5: A chlorophyll fluorescence induction kinetic curve with $10 \mu \mathrm{M}$ DCMU 


\section{List of Tables}

\section{Chapter 2}

Table 2.1 - Collection data for all anemones analysed in this study giving species, site collected, coordinates for the site and the date collected. 40

Table 2.2: Mean daily minimum and maximum temperature of the 5 years prior to the latitudinal collection of anemones throughout New Zealand. 41

Table 2.3: Identity and codes given for anemones that were processed and sequenced.

Table 2.4: Details of Symbiodinium types suggested by NCBI Database and

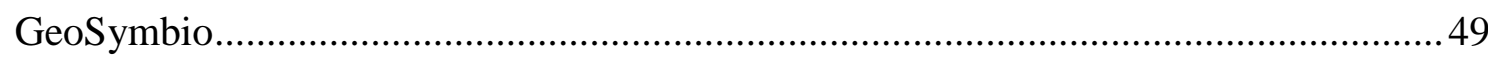

Table 2.5: Numbers of samples sequenced and used for molecular analysis................50

Table 2.6: Number of samples sequenced from each habitat 51

Table 2.7: Comparison of Symbiodinium identification from different molecular sequence analyses in host anemones that come from the NE Atlantic and the Mediterranean. 61

Table A1.3.1 Weather stations used to collect climate data. 110

Table A2.1.1 Coding to identify samples in this study

Table A2.2.1 Sequences of samples used for phylogenetic analysis 112

Table A2.3.2 Sequences of reference Symbiodinium types. 123

Table A5.1 Mean cell counts 141

Table A5.1.1-A5.1.2 Statistical data for cell density 142

Table A6.1.1-A6.1.5 Statistical data for $F_{v} / F_{m}$ 143 
Table A6.2.1-A6.2.2 Statistical data for $\Delta \mathrm{F} / \mathrm{F}_{\mathrm{m}}$,

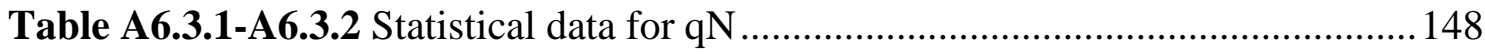

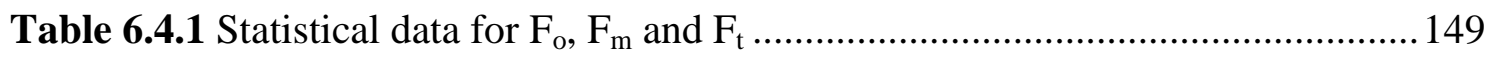

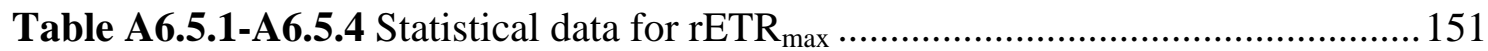

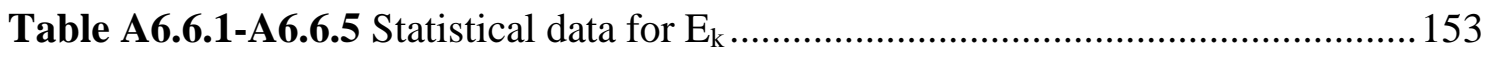

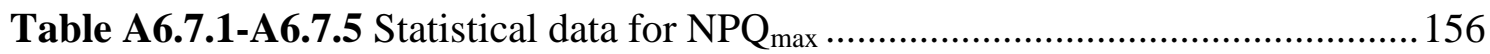




\section{$\underline{\text { Abbreviations }}$}

SSUrRNA

RFLP

ITS1 and ITS2

DNA

rDNA

PCR

qPCR

DGGE

SSCP

MYA

MAAs

BLAST

Med clade A

Amed

FSW

PSI and PSII

ATP

$\mathrm{NADPH}$

ROS

SOD

$\mathrm{ABH}$ small subunit ribosomal RNA

restriction fragment length polymorphism

non-coding internal transcribed spacer regions 1 and 2

deoxyribonucleic acid

ribosomal DNA

polymerase chain reaction

quantitative real-time PCR

denaturing gradient gel electrophoresis

single-strand conformation polymorphism

million years ago

mycosporine-like amino acids

Basic Local Alignment Search Tool

Symbiodinium Mediterranean clade A

Symbiodinium Amed

filtered seawater

photosystems 1 and 2

adenosine triphosphate

nicotinamide adenine dinucleotide phosphate

reactive oxygen species

superoxide dismutase

adaptive bleaching hypothesis 


\begin{tabular}{|c|c|}
\hline PAR & photosynthetically active radiation \\
\hline I-PAM & imaging-pulse amplitude modulated fluorometer \\
\hline $\mathrm{F}_{\mathrm{o}}$ & minimal fluorescence yield during dark adaptation \\
\hline$F_{m}$ & dark-adapted maximum fluorescence yield \\
\hline$F_{t}$ & instantaneous fluorescence yield \\
\hline $\mathrm{F}_{\mathrm{m}}$ & light-adapted maximum fluorescence yield \\
\hline $\mathrm{F}_{\mathrm{v}} \mathrm{F}_{\mathrm{m}}$ & $\begin{array}{l}\text { photosynthetic efficiency or dark-adapted maximum quantum } \\
\text { yield }\end{array}$ \\
\hline$\Delta \mathrm{F} / \mathrm{F}_{\mathrm{m}}$ & effective quantum yield \\
\hline$\phi_{\mathrm{PS}}$ & also effective quantum yield \\
\hline $\mathrm{qN}$ & the coefficient on non-photochemical quenching \\
\hline RLC & rapid light curve \\
\hline ETR & electron transport rate \\
\hline rETR & relative electron transport rate \\
\hline $\mathrm{rETR}_{\max }$ & maximum observed relative electron transport rate \\
\hline NPQ & nonphotochemical quenching \\
\hline $\mathrm{NPQ}_{\max }$ & highest nonphotochemical quenching value on the RLC \\
\hline $\mathrm{qE}$ & energy-dependent quenching \\
\hline $\mathrm{qT}$ & quenching due to state-transition \\
\hline qI & photoinhibitory quenching \\
\hline$\Delta \mathrm{pH}$ & $\mathrm{pH}$ gradient \\
\hline $\mathrm{E}_{\mathrm{k}}$ & saturation radiance \\
\hline
\end{tabular}




\section{Chapter 1 - Introduction}

\section{The Cnidarian-Dinoflagellate Symbiosis}

\subsection{Symbiosis}

\subsubsection{Definition and types of symbiosis}

The term "symbiotism" was originally used by Albert Bernhard Frank in 1877 to describe the relationship between fungi and algae in lichens (Frank 1877, Trappe 2005). He used the term to describe a mutually beneficial relationship between participating partners. The concept of symbiosis was expanded in 1879 by de Bary to include close relationships that can be detrimental to a partner; this therefore included parasitism (de Bary 1879, Trappe 2005). Symbiosis now generally describes an association formed between individuals (symbionts) of different species that "persists throughout a life time or for the major part of it" (Douglas 2010). Partnerships can be between closely related, confamiliar species (Baeza 2007) or distantly related taxa, such as dinoflagellates and cnidarians (Baeza 2007). These include the scleractinian (stony) corals and sea anemones.

Biological interactions between organisms are often described as being of an antagonistic nature; the most common being competition, predation or parasitism. Over time many organisms respond to these hostile biotic stresses and stressful abiotic situations, such as nutrient deficiency, by forming alliances (Douglas 2010). These alliances often result in enhanced fitness for the individuals and increased population size leading to ecological success for the species involved (Connell and Gillanders 2007). Often symbiosis also leads to the symbionts increasing their key biochemical functions, such as recycling nutrients in nutrient poor habitats or using energy from breaking down $\mathrm{H}_{2} \mathrm{~S}$ to convert $\mathrm{CO}_{2}$ into organic carbon around deep-sea vents, thereby allowing occupation of inhospitable environments and increasing the diversity and distribution of the species involved (Gast et al. 2003). 
There are three categories to describe symbiotic relationships. 'Mutualism' describes a relationship where both partners benefit from the association equally and where the alliance is reciprocal. The value of the benefits received from the partner are greater than the value of what has been given away (Douglas 2010). 'Commensalism' is where the relationship is beneficial to one partner but neither advantageous nor detrimental to the other. 'Parasitism' occurs where one partner benefits with a loss of function or health to the other. The association's impact on an organism varies with age, species, environmental conditions and the physical well-being of the partners (Douglas 2008). Therefore, in reality, the partnership can move along a gradient from mutualism through commensalism and onto parasitism as the situation changes, in which case the bond may be broken completely as the partnership breaks down.

Symbiosis can further be described as being 'obligate', in which at least one partner is dependent on the other for survival, a situation which has developed through coevolution. It can also be described as being 'facultative', where the individuals can survive and reproduce without their partners but are better off together (Baeza 2007). Usually the relative sizes of the partners are unequal where the host is larger than the smaller symbiont. The symbiosis can also be described by the symbiont's position; ectosymbiotic where the symbiont lives outside the host's body, such as the clownfish and a sea anemone, or endosymbiotic, where it is found within the host's body.

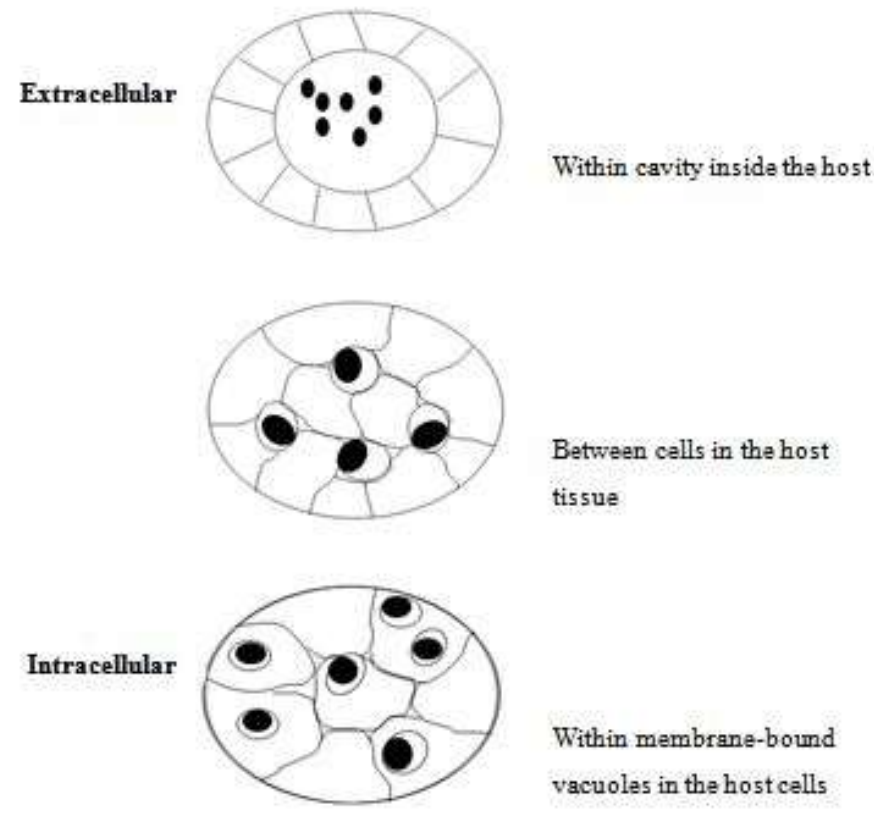

Figure 1.1: Forms of endosymbiosis as described by the position of the symbionts within the host 
The symbiont within the host can be extracellular, where it can be found within a body cavity or between cells in the host's tissue, or intracellular within membrane-bound vacuoles in the host's cells (Smith and Douglas 1987) (Figure 1.1). Intracellular symbiosis is therefore the sharing of the same cytoplasmic environment by two different genomes from separate evolutionary origins (Trench 1979).

\subsubsection{Cnidarian-dinoflagellate symbiosis}

The exact origins of the cnidarian-dinoflagellate symbiosis are unknown but the partnership has been hugely successful. Cnidarians include the corals, sea anemones and jellyfish (Figure 1.2).

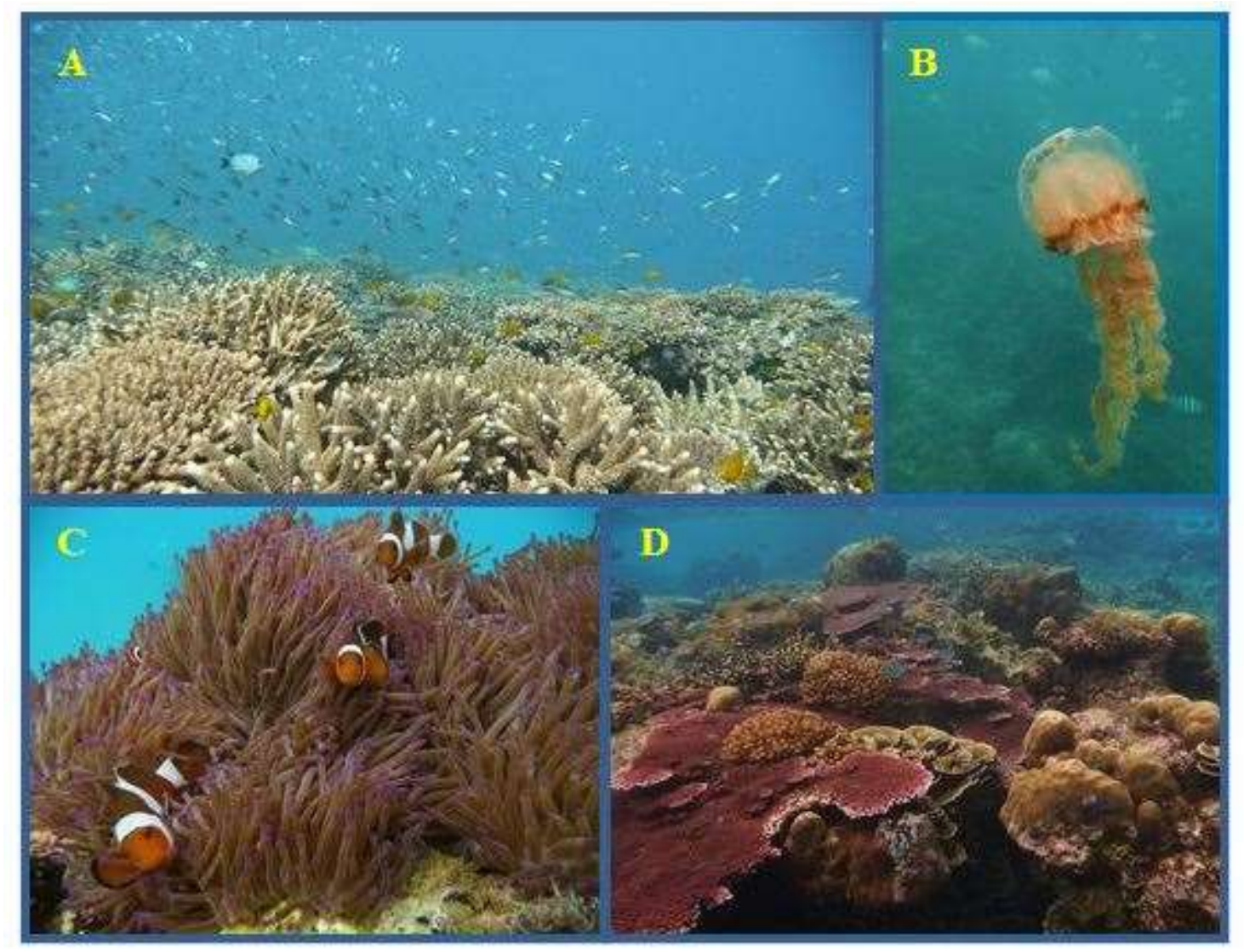

Figure 1.2: Cnidarians include corals, jellyfish and sea anemones. A) Coral reef at Heron Island, Australia; B) Jellyfish, C) clownfish and anemone and D) coral reef, all taken from reefs around Pulau Perhentians, Malaysia. 
There is a hypothesis that the modern coral-dinoflagellate symbiosis began in the Late Triassic period, about 235 MYA, from information collected by skeletal stable isotopes and organic matrix analyses of fossilized corals (Stanley and Swart 1995, Muscatine et al. 2005, Stanley 2006). These corals probably originated from soft, non-reef-building anemone-like ancestors (Stanley and Fautin 2001). This photo-endosymbiotic relationship was important in shaping the co-evolution of the host and symbiont, leading to ecological success, especially for scleractinian corals in nutrient-poor waters (Stanley 2006). The sharing of nutrients between the partners provided a competitive metabolic advantage (Smith et al. 2005) leading to enhanced growth and laying down of calcified skeleton. This has led to the domination of tropical reefs by stony corals (Stanley 2006).

\subsubsection{The symbiont and host}

The photosynthetic unicellular dinoflagellate algae that form symbiotic relationships with cnidarians are mostly of the genus Symbiodinium (Baker 2001), though at least one species belongs to the genus Gymnodinium (Karako-Lambert et al. 2004). These microscopic algae are commonly known as 'zooxanthellae' due to the golden-brown colour that they give to their coral hosts (Brown et al. 2002, Perez 2007) (Figure 1.3 E). They can have two forms, a coccoid vegetative cyst of 5-15 $\mu \mathrm{m}$ in diameter and a motile zoospore (Stat et al. 2006). The coccoid Symbiodinium cells contain a large nucleus, in which the chromosomes are permanently condensed, a pyrenoid body and disk-shaped chloroplasts containing the photopigments chlorophylls $a$ and $c 2$, and xanthophylls unique to dinoflagellates, peridinin, dinoxanthin and diadinoxanthin (Jeffrey and Haxo 1968, Hackett et al. 2004). This haploid vegetative cyst (Santos and Coffroth 2003) is the form that the Symbiodinium cell takes when in an endosymbiotic relationship.

The cnidarian host has a very simple body plan composed of two tissue layers, an outer epidermis and an inner endodermis or gastrodermis. These are separated by an acellular mesogloea (Perez 2007, Weis et al. 2008). The body form is a tubular shape or polyp with a single digestive tract continuous with the hollow feeding tentacles. 


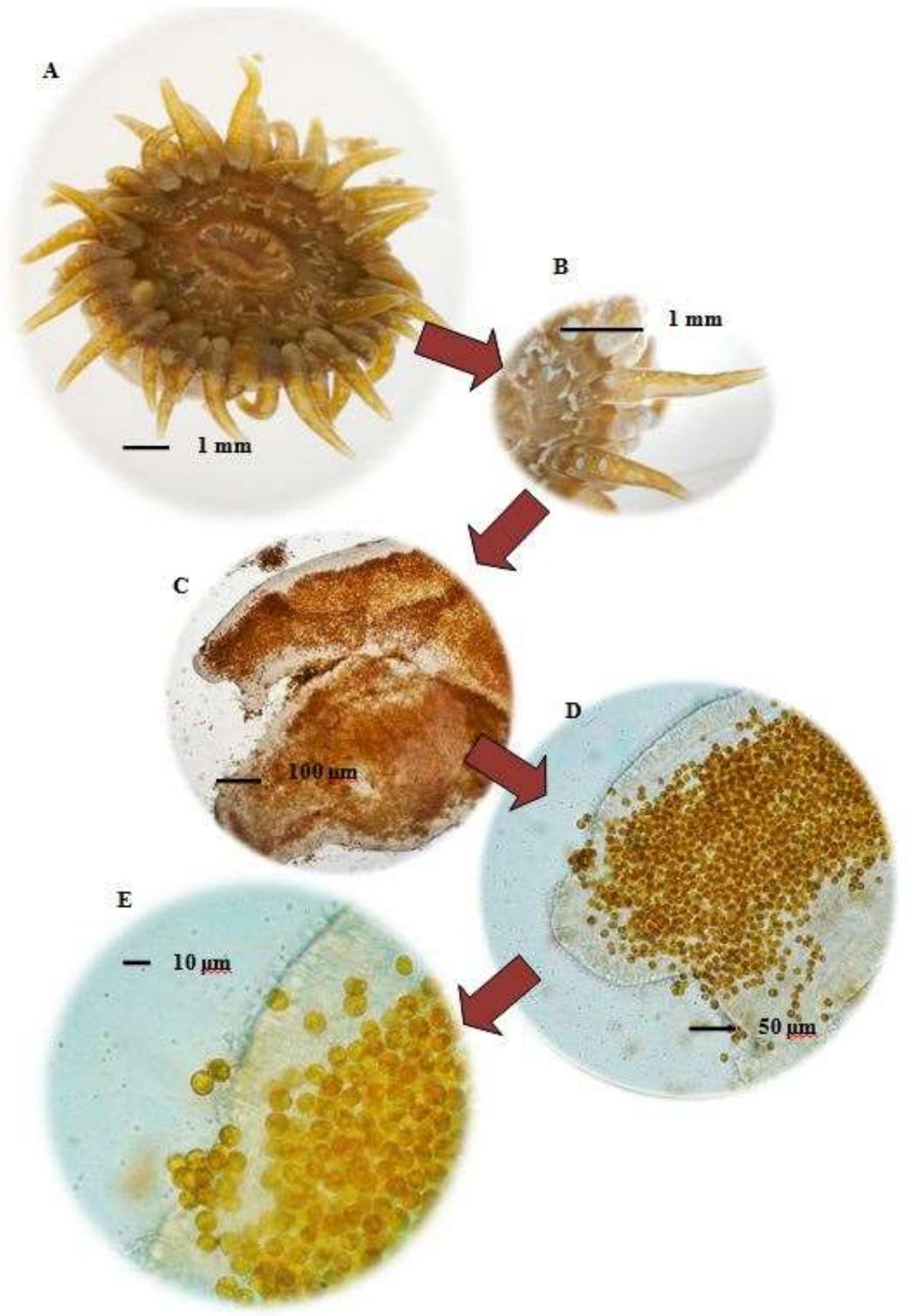

Figure 1.3: The study organism Anthopleura aureoradiata, with its associated Symbiodinium spp. A) A. aureoradiata under a microscope; B) close-up of tentacles illustrating the brown colouration of the host but originating from the Symbiodinium cells; C) and D) a tentacle squash illustrating the dense assembly of Symbiodinium cells located within the endodermal tissue; E) Symbiodinium cells escaping the squashed tentacle, showing their coccoid shape and golden-brown colouration. 
The Symbiodinium cells are localized within the endodermal cells (Grant et al. 2003, Yellowlees et al. 2008) in vacuoles surrounded by a host-derived membrane (Rands et al. 1993). Between this membrane and the algal symbiont there may be other membranes that are symbiont-derived (Wakefield and Kempf 2001). These membranous layers are composed of sloughed plasma membranes, thecal vesicles and plates. Altogether they are known as the symbiosome membrane complex (Kazandjian et al. 2008) and the entire vacuolar structure is called the symbiosome (Rands et al. 1993, Wakefield and Kempf 2001) (Figure 1.4). All communication between host and symbiont has to cross these membranes.

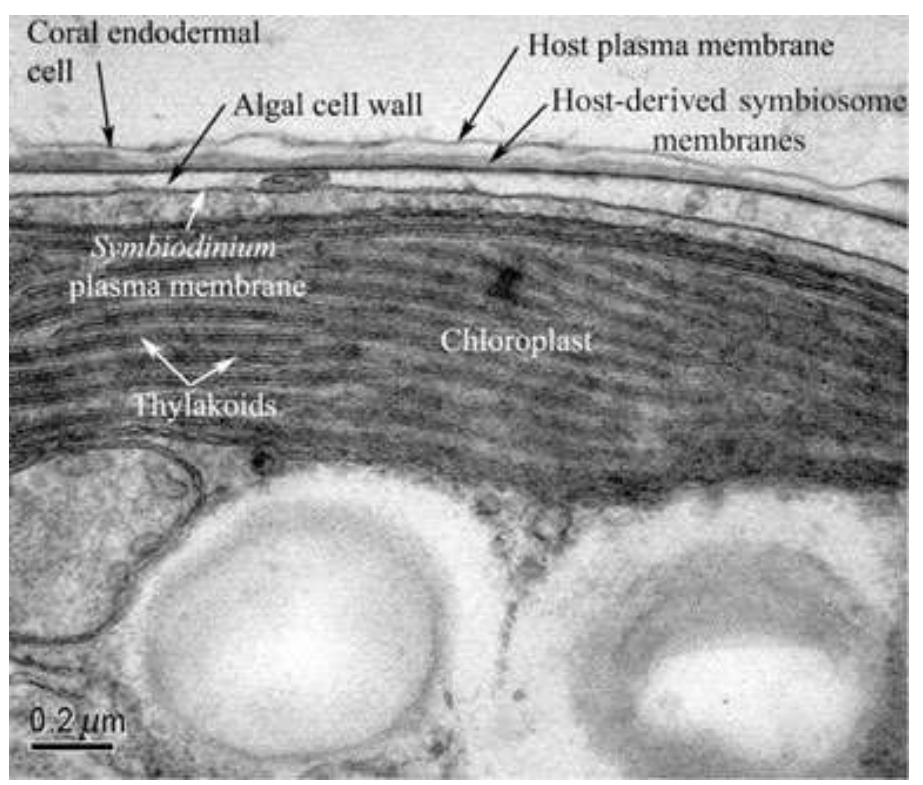

Figure 1.4: The arrangement of algal cellular structures, algal plasma membrane and cell wall in the cnidarian-Symbiodinium symbiosis, all surrounded by the host-derived membrane (Yellowlees et al. 2008).

\section{$\underline{\text { 1.2 Cellular basis of cnidarian-dinoflagellate symbiosis }}$}

\subsubsection{Establishment of the symbiosis}

Cnidarian hosts acquire their endosymbiont cells either vertically (i.e. passed on from their parents) or horizontally from the surrounding environment (Davy and Turner 2003). An estimated $85 \%$ of tropical corals acquire their symbionts horizontally during the host planula larva or juvenile polyp stages (Schwarz et al. 2002, Dunn and Weis 2009) whereas in temperate waters, maternal transmission is thought more likely to 
occur due to the scarceness of Symbiodinium cells in the water column (Muller-Parker and Davy 2001). Horizontal transmission is dependent on there being a plentiful source of free-living Symbiodinium cells (Baker 2003) which have been released by other symbiotic invertebrates or from faeces of corallivorous predators (Muller-Parker 1984). Although there is the potential for the host to associate with many different species of Symbiodinium, there appears to be a high degree of specificity between host species and symbiont, and often with only one species of Symbiodinium per host (Dunn and Weis 2009). This suggests that ecological processes may have involvement in determining the extent of specificity or that there has to be a process of recognition between the cells of the symbiont and the host. This progressive process of identification and selection has been termed 'winnowing' (Dunn and Weis 2009, Voolstra et al. 2009) and probably involves the recognition of different glycoproteins, lectins and receptors on the host and symbiont membranes (Lin et al. 2000, Wood-Charleson et al. 2006, Kvennefors et al. 2008, Kerrigan and Brown 2009, Logan et al. 2010).

Once uptake of the genetically foreign symbiotic organism has occurred, there has to follow some form of resistance or avoidance of the host cell's defence (Fitt and Trench 1983). Unwanted Symbiodinium cells within host cellular vacuoles are either recognised and broken down or the whole host cell is destroyed by apoptosis (Dunn and Weis 2009). Vacuoles containing required symbionts appear to avoid lysosomal fusion and may do so by membrane enzymes (Rab proteins) of the host and symbiont interacting (Chen et al. 2003) or by control in the host cell of vesicular trafficking (Dunn and Weis 2009). This prevents phagosomal maturation and stops the processes leading to vacuole degradation (Vieira et al. 2002). Host cells with appropriate Symbiodinium cells stop caspase activity, which controls the innate immune cellular pathways, thereby evading apoptosis (Dunn and Weis 2009).

\subsubsection{Maintenance of the symbiosis}

The success of this symbiotic relationship, particularly in the tropics, is based upon its enhanced nutritional potential. Here the host potentially receives more than $100 \%$ of its energy requirements from organic carbon fixed by symbiont photosynthesis (Steen and Muscatine 1984, Falkowski et al. 1993, McCloskey and Muscatine 1994, Wang and Douglas 1998). It is thought that the symbionts recycle respiratory $\mathrm{CO}_{2}$ and other host 
metabolic waste (e.g. inorganic nitrogen and phosphorus) and translocate up to $99 \%$ of their photosynthate (e.g. glycerol glucose, amino acids and fatty acids) to the host (Muscatine 1967, Muscatine et al. 1989, Davy et al. 1996, Hoegh-Guldberg and Jones 1999, Muscatine et al. 2005, Perez 2007). The host uses this for respiration, growth, calcification, mucus secretion, reproduction and regeneration (Smith and Bernays 1991, Hoegh-Guldberg 2004, Furla et al. 2005, Obura 2009). One or both partners may express regulatory mechanisms that control the amount of photosynthate translocated, used and shed by the host, and that manage biomass regulations between the two (Obura 2009), but these mechanisms are poorly understood (Davy et al. 2012).

Most Cnidaria live in nutrient-poor tropical waters and have developed adaptations for assimilating and conserving nitrogen. Ammonium resulting from digestion and metabolism of ingested food by the host is not excreted but re-assimilated by the symbiont and recycled as amino acids back to the host (Furla et al. 2005). This is the basis of the nitrogen recycling hypothesis where the sharing of nitrogen benefits both partners in symbiosis (Wang and Douglas 1998). In addition, a nitrogen conservation hypothesis proposes that photosynthetically-fixed carbon will be used preferentially in animal respiration instead of amino acids. The conserved nitrogenous compounds in the animal tissue can then be used instead for animal growth (Smith and Bernays 1991). The conservation of nitrogenous compound in the animal tissue would promote the persistence of the symbiosis in low-nitrogen environments (Wang and Douglas 1998).

\subsubsection{Breakdown of the symbiotic partnership}

The symbionts are present in extremely high densities within the host animal, and may fluctuate over time in response to external environmental factors such as temperature change, salinity, increased sedimentation, pollution, nutrient levels, infection by pathogens and changes in light quality (increased visible light or UV radiation) (Brown 1997, Gates and Edmunds 1999, Hoegh-Guldberg and Jones 1999, Douglas 2003, Mayfield and Gates 2007). Many of these factors have a synergistic effect on the interactive relationship between the symbiotic partners. Changes to these environmental factors can stress the symbiotic balance to the point where the partnership breaks down, leading to loss of the symbionts from the host cells. In corals this is called coral bleaching as they lose their dinoflagellate pigmentation and colour. The bleaching 
process affects conspecific coral colonies and various species of corals differently, which may be due to the difference of Symbiodinium types within (Baker 2001, Ulstrup and Van Oppen 2003). If conditions are extreme enough or continue long enough to prohibit new partnerships to be formed, bleaching can lead to death of the coral polyps as their endosymbionts are essential for their wellbeing (Hoegh-Guldberg 1999, Weis 2008). There are five mechanisms suggested that explain the loss the symbiont (Edmunds and Gates 2003, Weis 2008) (Figure 1.5).

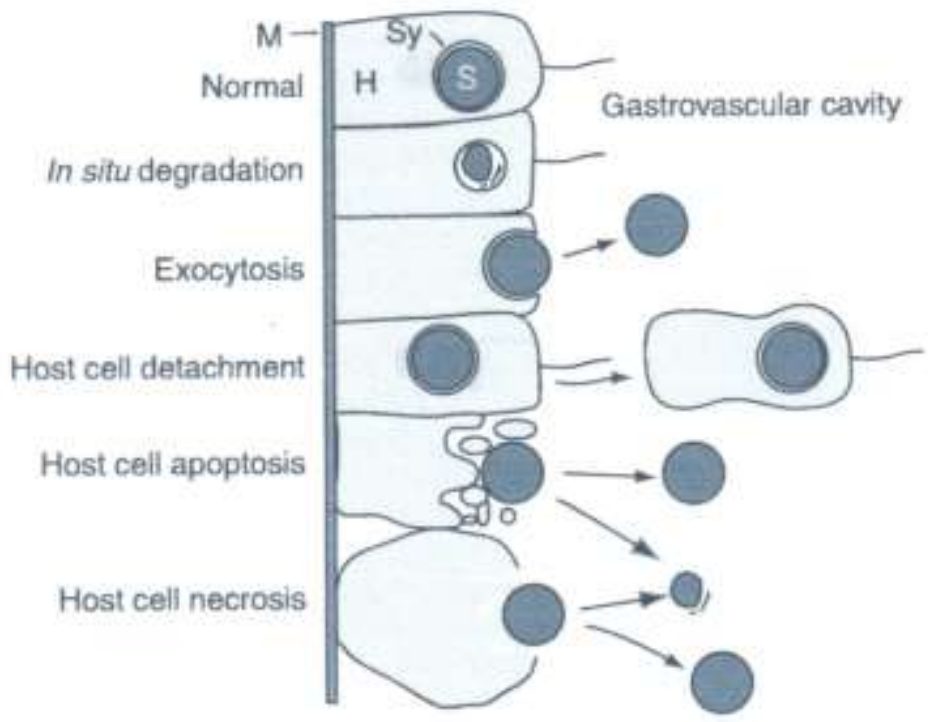

Figure 1.5: Normal host cells $(\mathrm{H})$ are anchored to the acellular mesogloea (M). Symbionts (S) are contained within host vacuoles or symbiosomes. The five mechanisms of cellular breakdown are; 1) Insitu degradation via programmed cell death and autophagy of the symbiont by the host cell (Weis 2008, Dunn and Weis 2009); 2) Exocytosis or expulsion of the symbiont (Fang et al. 1997); 3) Host cell detachment from the mesogloea (Brown et al. 1995, Baghdasarian and Muscatine 2000, Sawyer and Muscatine 2001); 4) Host cell apoptosis, a form of programmed cell death (Lesser and Farrell 2004, Strychar et al. 2004, Dunn et al. 2007); and 5) Necrosis which is an uncontrolled host cell death from external stress factors, which can be reversed, if the stress is decreased (Dunn et al. 2004). Figure taken from Weis (2008). 


\section{$\underline{1.3 \text { Genetic diversity of Symbiodinium }}$}

\subsubsection{Molecular identification within the genus Symbiodinium}

Early classification of the members of the genus Symbiodinium was based on morphology. Study of the coccoid and non-motile symbiotic cells found them to be so similar that they were described as one, pandemic species, Symbiodinium microadriacticum Freudenthal (Freudenthal 1962, Taylor 1974). However, since then, molecular, physiological and ultra-microscopical analyses have determined that there is a diverse range of Symbiodinium types. Genetic studies in particular have shown that there is a tremendous diversity in this genus. Using the small subunit ribosomal RNA (SSUrRNA) (18S), Rowan and Powers (1991) were the first to divide the genus into 3 large groups or clades, each containing about half a dozen types based on restriction fragment length polymorphism (RFLP) and sequencing. Further work using the large subunit region (28S) (Rowan 1998), chloroplast large subunit (23S) (Santos et al. 2002) and mitochondrial protein-coding gene (cytochrome oxidase subunit 1 (cox1)) (Takabayashi et al. 2004) led to more cladal divisions. Studies using the non-coding internal transcribed spacer regions (ITS), consisting of ITS1, 5.8S and ITS2, led to a vast expansion of recognised types within the genus (Hunter et al. 1997, Baillie et al. 2000, LaJeunesse 2001) (Figure 1.6).

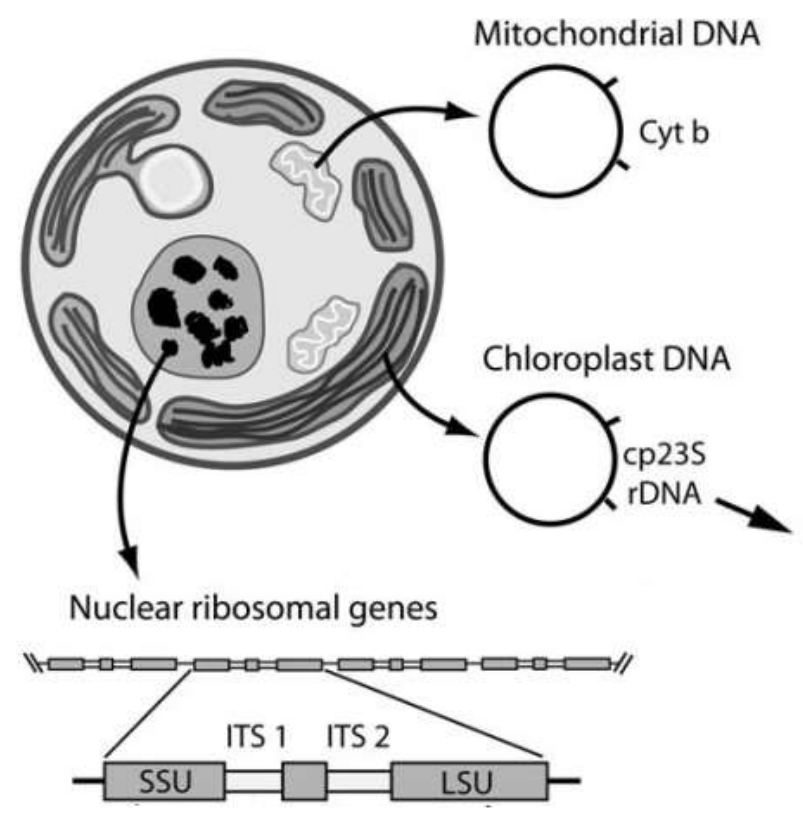

Figure 1.6: Schematic of nuclear and organelle genomes targeted for molecular differentiation of the genus Symbiodinium (Sampayo 2009). 
The ITS regions are part of the eukaryotic nuclear rDNA gene and lie between the $18 \mathrm{~S}$ small ribosomal subunit and the 28S large ribosomal subunit. A feature of this sequence is that there is a high level of divergence between species, but it is well conserved within a species, and therefore makes a suitable marker for low level phylogenetic analyses (Schultz et al. 2006, Stat et al. 2006).

After PCR amplification of the particular region of interest, rapid resolution of distinct Symbiodinium types can be made on denaturing gradient gel electrophoresis (DGGE) or single-strand conformation polymorphism (SSCP) non-denaturing gels. ITS1 and ITS2 amplification have been widely used to differentiate between types within a clade. The term "type" is used to name genetically similar members of the genus Symbiodinium. These have unique genetic sequences at any particular locus of a gene or DNA sequence (Coffroth and Santos 2005). SSCPs distinguish between different types by relying on the folding of single-stranded DNA into specifically shaped "secondary structures" where nucleotide bases will match with only one of the other base types (adenine with thymine and cytosine with guanine), leaving some of the DNA strand free from a partner (Figure 1.7). The secondary structures migrate through the gel according to their shape and size. DNA strands of different sequences will generally not assume the same shape and so have distinct gel mobilities and final position (Sunnucks et al. 2000).

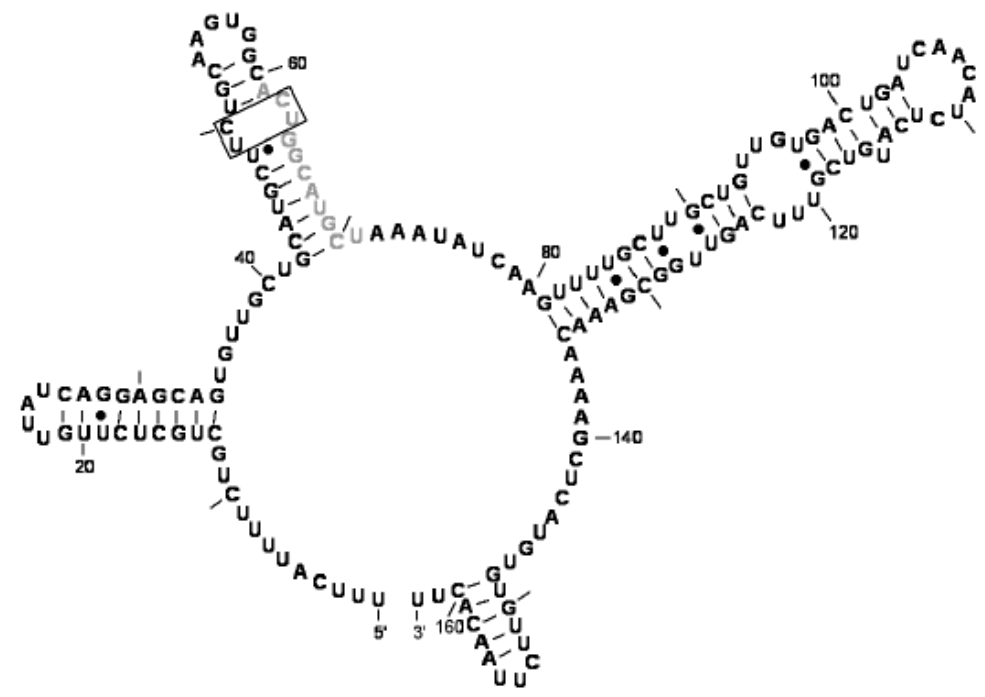

Figure 1.7: Proposed ITS2 secondary structure for Symbiodinium type A1 (Hunter et al. 2007) 
Nine clades have now been recognised (Clades A-I) (Pochon and Gates 2010) with over 400 ITS2 types identified from sequencing and gel profiles (Silverstein et al. 2012). Clades A-H are found in scleractinian corals, A-D more commonly so (Baker 2003, LaJeunesse et al. 2010), clade $\mathrm{E}$ in anemones and some corals (LaJeunesse and Trench 2000), and clades C, D and F to I in Foraminifera (Pochon and Gates 2010). Radiation within the genus Symbiodinium, from the first clade, clade A, occurred about 50 MYA at the beginning of the Eocene period, probably relating to the divergence and radiation of the scleractinian corals at this time (Pochon et al. 2006) (Figure 1.8). Ancient ancestors of clade A may have been present 65 MYA (Tchernov et al. 2004) as symbionts within Palaeocene corals which managed to pass through the time-period of the K-T boundary (Pochon et al. 2006).

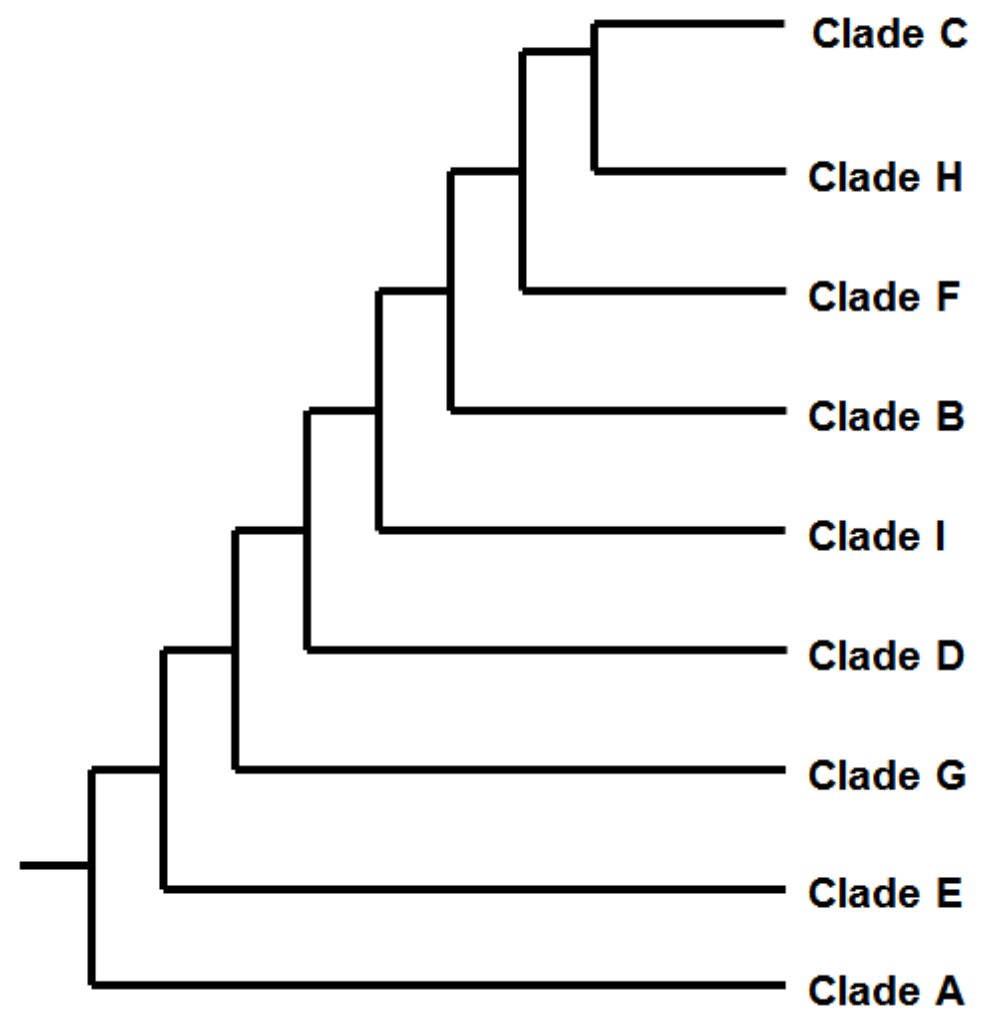

Figure 1.8: The phylogenetic relationships between the major clades of Symbiodinium. The cladal tree is a consensus from a maximum likelihood phylogram based on molecular analysis by Pochon et al (2006 and 2010). 
Several studies have suggested that there are functional differences between the different clades and that prediction of habitat and geographical distribution, and tolerance to different irradiance and temperature regimes can be made from the genetic type (Rowan 1998, Toller et al. 2001). Clade A have been described as "invasive/opportunistic/parasitic", B as "sunspecialists, C as "shade specialists" and E as "stress tolerant" (Rowan 1998, Toller et al. 2001, Savage et al. 2002b). Differences in benefits for the host have been found where one clade has been reported to supply different proportions of metabolites and MAAs in photosynthate than another (Loram et al. 2007, Reynolds et al. 2008). However, cladal differences cannot always be used as a prediction of functionality, as molecular types within a clade have been found to present varying levels of thermal (LaJeunesse et al. 2003) and irradiance tolerance (Savage et al. 2002b).

\subsubsection{Biogeographic and ecological variability in diversity}

The increasing knowledge of molecular diversity within the genus Symbiodinium has revealed not only the extent of specificity between hosts but also the wide-scale distribution and patterns made by both global and small-scale ecological differences, such as depth, light and temperature (Sampayo et al. 2007, McDonald et al. 2008, Silverstein et al. 2012). Members of clade $\mathrm{C}$ dominates in the tropics with a transition to clades A, B and D with increasing latitude (Toller et al. 2001, Van Oppen et al. 2001, Savage et al. 2002a). Also, clade C types are present in most coral hosts in the Pacific whereas clade B dominates in the Caribbean with some clade A (LaJeunesse et al. 2003).

A single host species may have a change of clade with increasing latitude. This change could be attributed to changes in temperature, irradiance or turbidity (McDonald et al. 2008). For example the coral Plesiastrea versipora hosts clade $\mathrm{C}$ at low latitudes and $\mathrm{B}$ at the high-latitude margins of its distribution (Rodriguez-Lanetty et al. 2001). Similarly, from $6^{\circ} \mathrm{N}$ in Thailand to $35^{\circ} \mathrm{N}$ in Japan the symbionts in Oulastrea crispate change from being both clade C and D to just being clade C (Lien et al. 2007). Differences also occur at a sub-cladal level. In southeast Africa a shift with latitude was observed between six Symbiodinium types within clade C in the coral Stylophora pistillata (McDonald et al. 2008). 
In the temperate anemones Anthopleura elegantissima and Anthopleura xanthogrammica, not only is there a change in Symbiodinium type with latitude but also a change in the ratio between Symbiodinium symbionts and a green symbiotic alga ('zoochlorella') (LaJeunesse and Trench 2000, Secord and Augustine 2000). Zoochlorellae are restricted to northern latitudes and coexist with Symbiodinium muscatinei (clade B), whereas further south the anemones host just Symbiodinium californium (clade E). Midway there are mixed populations of both Symbiodinium species (LaJeunesse and Trench 2000). Laboratory experimental evidence, field observations and transplant experiments have shown that the changes are influenced by light and temperature. Zoochlorellae grow and photosynthesise more at lower temperatures and irradiances, and zooxanthellae at higher temperatures and irradiances (Secord and Augustine 2000, Verde and McCloskey 2007). Therefore a change in composition was also found with shore height, with zoochlorellae more abundant on the lower shore and zooxanthellae on the higher shore (Bates 2000).

Shore height and depth differences in clades also occur on tropical reefs. Clades A and B were found in Montastraea annularis in the Caribbean in shallow, well-illuminated sites, but in deeper more dimly-illuminated sites the coral hosted clade C (Baker 2003). Depth, light and temperature influenced a change of clades in the anemone Condylatis gigantea in Bermuda, with clade A inshore in variable temperatures and high light, and clade B offshore, with mixed populations in between (Venn et al. 2008b). These authors found that population composition also changed due to season with clade A more prevalent in the summer and B in the winter.

There are also examples of Symbiodinium types that demonstrate a widespread global distribution, with some Symbiodinium isolates from the Caribbean being identical to some from the Red Sea or Western Pacific (LaJeunesse 2001). A single 'Temperate A' ribosomal haplotype is widely distributed in anemones, gorgonians, scleractinian, hydrozoans and hexacorals in the Mediterranean and north-eastern Atlantic (Bythell et al. 1997, Savage et al. 2002a, Visram et al. 2006, Focioli et al. 2011). Widely distributed Symbiodinium types tend to be host-generalists, with the ability to be in symbiosis not only with different cnidarian species but also with species from other 
phyla such as molluscs. These are also, phylogenetically, more ancestral than the Symbiodinium types that have specialised hosts (LaJeunesse et al. 2004).

\subsection{Photophysiology of Symbiodinium}

Photosynthesis by the phototrophic endosymbionts brings in nutritional benefit for the host but can also be a potential source of stress. The most significant is when a warming of sea surface temperatures acts synergistically with high solar radiation to cause photoinhibition (long-term reduction in photosynthetic capacity) and damage to the chloroplast and photosystem apparatus (Weis 2008, Vidal-Dupiol et al. 2009). In optimum conditions Symbiodinium cells can undergo high rates of photosynthesis, but there has to be a balance between the rates of collecting energy from light and the use of the energy made (Venn et al. 2008a). Maximum photosynthesis and production of organic carbon molecules rely upon an efficient photosynthetic apparatus.

Photosynthesis in the Symbiodinium cell takes place in two stages: The energy transduction reaction (light stage) and the carbon fixation reaction (dark stage or CalvinBenson Cycle). Two photosystems (PS) are involved in the light reaction, photosystem I (PSI) and photosystem II (PSII). Each PS constitutes an assembly of about 250-400 pigments and consists of closely-linked components, an antenna complex that gathers light and passes it onto a reaction centre. The reaction centre is made up of proteins and pigment molecules, chlorophylls $a$ and $c_{2}$ and peridinin, that convert light energy into chemical energy (Iglesias-Prieto 2005).

PSII, within all plants and algae, is primarily located in the grana thylakoid membranes and PSI is almost entirely in the stroma thylakoids and outer portion of the grana. Therefore the two centres are spatially separated, and electrons passing from PSII to PSI require carriers such as plastoquinone and plastocyanin to pass them on via the electron transport chain. Energized electrons from PSII synthesise adenosine triphosphate (ATP) as they pass down the chain and are replaced by electrons from water molecules being split. A product of this split is $\mathrm{O}_{2}$ gas. Light also excites PSI and the electrons from there are stored in nicotinamide adenine dinucleotide phosphate (NADPH). The stored electrons are replaced from PSII. ATP and NADPH are passed into the stroma to provide energy for the fixation of $\mathrm{CO}_{2}$ by the enzyme ribulose biphosphate carboxylase 
oxygenase (Rubisco) (Raven et al. 2005). Symbiodinium chloroplasts also have a Form II Rubisco, which is not efficient at discriminating between $\mathrm{O}_{2}$ and $\mathrm{CO}_{2}$, and therefore needs a high concentration of $\mathrm{CO}_{2}$ to be maintained to ensure continuous carbon fixation in the Calvin-Benson cycle (Wooldridge 2009).

Environmental stress affects the photophysiology of the Symbiodinium cell and leads to photoinhibition by dysfunction of the photosynthetic apparatus in three inter-related mechanisms in the chloroplast (Venn et al. 2008a, Nesa and Hidaka 2009) (Figures 1.9). These are damage to the D1 protein in PSII, disruption to the electron transport chain (energetic uncoupling) in the thylakoid membrane, and impairment of the carbon fixation cycle. All three sites of disruption potentially lead to synthesis of reactive oxygen species (ROS).

\section{AMBIENT}

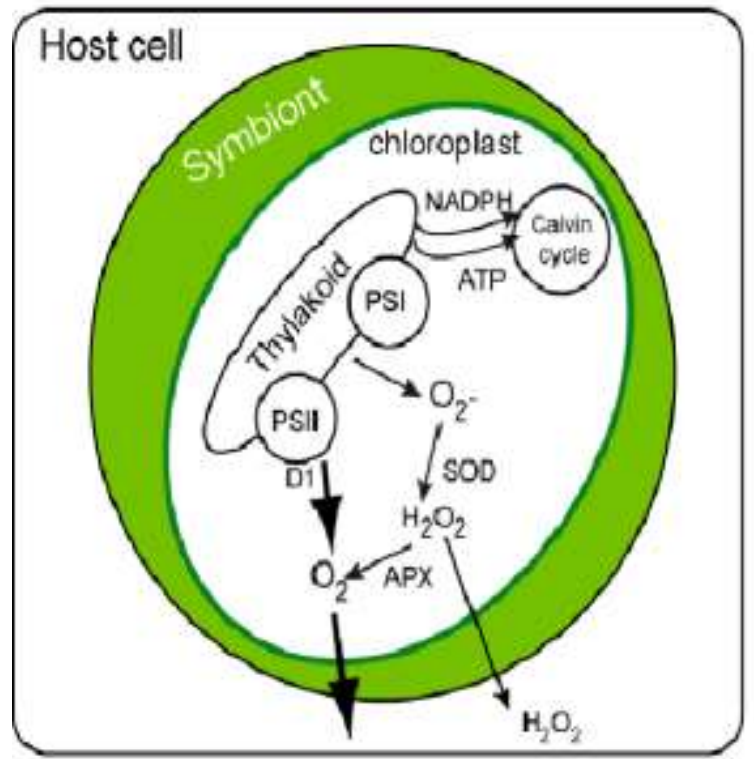

Excess heat

\& light
STRESSED

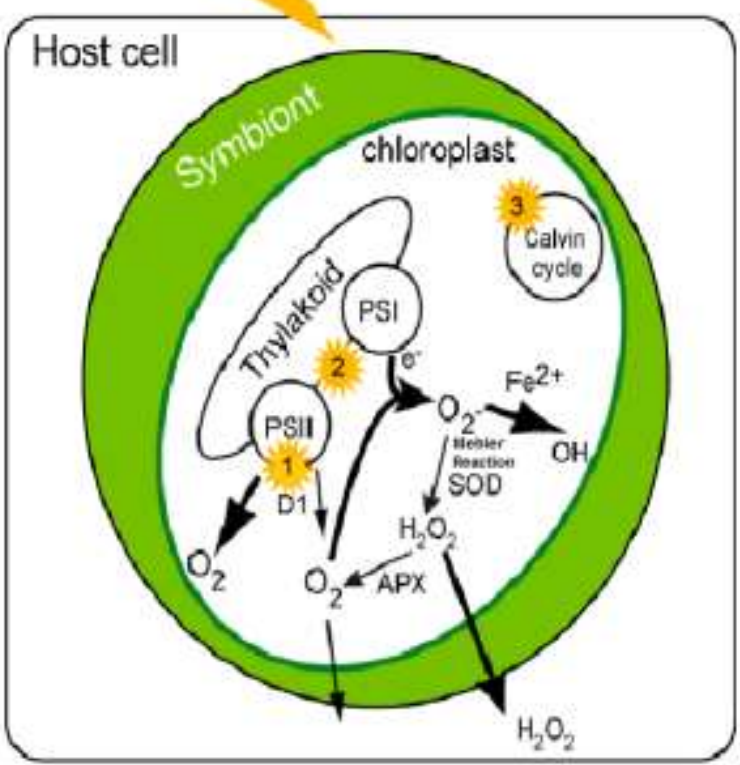

Figure 1.9: Representation of photosynthesis under ambient conditions and excessive temperature and light. When functioning optimally, PSI and PSII on the thylakoid membrane produce oxygen which diffuses to the host. Most reactive oxygen species (ROS) produced are converted back to oxygen. There are three proposed impacts of elevated temperature and light on the photosystem of Symbiodinium cells (shown with yellow flashes): 1) Dysfunction of PSII and degradation of the D1 protein; 2) energy uncoupling in the thylakoid membranes; 3) impairment of the Calvin-Benson Cycle leading to overproduction of ROS. Figure adapted from (Wicks 2009), which was adapted from Weis (2008) and Venn et al. (2008). 
D1 are rapid-turnover proteins that bind the components of the reaction centres and electron transport chain together, and are part of the water-splitting complex in PSII (Miyao et al. 1995). They are a vulnerable component of PSII and have the highest rate of turnover of all the chloroplast proteins. The D1 proteins are degraded by high light but are constantly being repaired and replaced. Under normal conditions the capacity for repair equals the rate of damage but, under stress, the rate of damage exceeds that of repair (Smith et al. 2005, Takahashi et al. 2009). High temperatures may damage the repair mechanism itself (Takahashi et al. 2004), though recent studies have found that this is probably not the case (Hill et al. 2011). These authors found that the repair mechanism did increase the repair rate, but not enough to keep up with damage. Degradation occurs by there being more energy in the photosystems than can be used in the Calvin-Benson Cycle. The excess energy comes from a reduction in electron transport combined with continued absorption of light (Lesser 2006). The electrons contribute to the production of ROS and these powerful oxidants increase damage to the photosystem components (Franklin et al. 2005, Venn et al. 2008a). If these are produced in the plastiquinone binding sites as suggested, they would specifically degrade the D1 protein (Miyao et al. 1995).

It has also been suggested that heat and light initially damage the Calvin-Benson Cycle so that carbon fixation decreases. This causes a downstream impact damaging PSII (Jones et al. 1998). These authors suggested that the site of damage may be the enzyme Rubisco, which would lead to reduced carboxylation resulting in reduced consumption of ATP and NADPH from the light reaction. This would then lead to increased free electrons, which reduce oxygen, producing more ROS. However, Lesser and Farrell (2004) suggested that both photochemistry and carbon fixation are affected simultaneously in a feedback loop increasing the damage to PSII. The ROS formed also go on to inhibit the function of Rubisco, adding to the loop (Lesser and Farrell 2004).

Heat and high light may also damage the thylakoid membranes directly (Weis 2008). This leads to uncoupling of the electron transport chain and allows leakage of protons (Tchernov et al. 2004). As a result there is a cessation of ATP and NADPH synthesis but generation of electrons continues by light exciting PSI and PSII, with water molecules still being split. The $\mathrm{O}_{2}$ generated by PSII can then react with the 
photochemically-generated electrons in PSI to form more ROS in the Mehler reaction (Arora et al. 2002, Venn et al. 2008a). The ROS then oxidize the thylakoid membrane lipids (Tchernov et al. 2004). Differences in genetically predetermined lipid biochemistry between Symbiodinium types may be one of the reasons for variations in stress susceptibility (Franklin et al. 2005, Diaz-Almeyda et al. 2011). Sensitive types have lower concentrations of saturated polyunsaturated fatty acids which are more predisposed to attack from ROS (Berkelmans and van Oppen 2006). A higher concentration of the fatty acids allows the thylakoid membrane to remain more fluid, allowing for migration of energy-dissipating pigments.

The Mehler reaction may act as a protective mechanism providing an electron sink to help prevent the PSII electron acceptors from becoming fully reduced at high light intensities (Smith et al. 2005, Venn et al. 2008a). The Mehler reaction reduces $\mathrm{O}_{2}$ to produce superoxide radicals. These are converted rapidly within the chloroplast into hydrogen peroxide $\left(\mathrm{H}_{2} \mathrm{O}_{2}\right)$ by superoxide dismutase (SOD): $\mathrm{H}_{2} \mathrm{O}_{2}$ is then detoxified into water by ascorbate peroxidase (Downs et al. 2002, Smith et al. 2005). Under most conditions the rate of detoxification should exceed the rate of ROS generation but when under stress, the enzymes become overworked and cannot detoxify the ROS, which accumulate (Franklin et al. 2005). $\mathrm{H}_{2} \mathrm{O}_{2}$ then can readily diffuse across membranes disrupting other cell reactions (Tchernov et al. 2004, Lesser 2006) and can react with ferrous iron $\left(\mathrm{Fe}_{2}{ }^{+}\right)$to form the most reactive ROS, a hydroxyl radical ( $\mathrm{OH}$ ) (Weis et al. 2008). $\mathrm{OH}$ attacks all biological molecules as it diffuses across the cell, oxidising membrane lipids and denaturing proteins and nucleic acids (Lesser 2006, Csaszar Jr et al. 2009).

As well as the ROS leaking from the damaged symbiont, there are also ROS produced from the host's mitochondria, damaged too from excessive heat and light. The host also has an antioxidant response which can become overwhelmed (Richier et al. 2006). The combined ROS cause photoinhibition and cellular damage to both host and symbiont, and lead to cell apoptosis and necrosis (Lesser and Farrell 2004). $\mathrm{H}_{2} \mathrm{O}_{2}$ is also known to act as a signal for digestion, apoptosis and exocytosis of the Symbiodinuim cell by activating a mitogen-activated protein kinase (MAPK) or caspase-signalling cascade (Smith et al. 2005, Perez 2007, Tchernov et al. 2011). Tchernov et al. (2011) found that 
the addition of caspase inhibitors prevented bleaching and death even in sensitive corals. ROS could also act as a signal to stimulate the host to produce nitric oxide (NO) during heat stress (Perez and Weis 2006). These authors proposed that the symbionts usually modulate the host's immune system and so act as a controlled infection, 'hiding' themselves from the host's immune system. By signalling their presence with the production of ROS when damaged, which triggers NO production, the host initiates an immune response to eliminate them, as it would a foreign pathogen (Perez and Weis 2006, Weis et al. 2008).

As radiance from the sun can be highly variable, the Symbiodinium cell must have efficient protective mechanisms to be able to photoacclimate, so optimizing productivity from the light available. The symbiont is able to dissipate excess light energy in PSII via the xanthophyll cycle in nonphotochemical quenching (NPQ) (Brown et al. 1999, Obura 2009). Protection by the NPQ mechanism occurs within seconds but can last for hours. NPQ can be divided into three component mechanisms: energy-dependent quenching (qE); quenching due to state-transition (qT); and photoinhibitory quenching $(\mathrm{qI})$.

$\mathrm{qE}$ is a feedback control mechanism induced when the absorbed light intensity is greater than what can be used by electron transport in the thylakoid membrane. Too much light absorption leads to accumulation of protons in the thylakoid lumen. This results in thermal dissipation of absorbed energy via the pigment antenna of PSII. This is in turn dependent on the energisation of the thylakoid membrane or transthylakoid $\mathrm{pH}$ gradient $(\Delta \mathrm{pH})$ (Horton and Hague 1988). The gradient can relax very quickly (seconds to minutes) (Muller et al. 2001). During induction of $\mathrm{qE}$, the build-up of $\Delta \mathrm{pH}$ activates the synthesis of diatoxanthin (Goss et al. 2006), a xanthophyll pigment specific to dinoflagellates (Jeffrey and Haxo 1968). The synthesis of diatoxanthin decreases the rate that photons are passed onto the PSII RCs, passing on the energy as heat (Smith et al. 2005)

qT quenching, or state transition, is the ability to vary the distribution of excitation energy between the two photosystems, so maximizing the overall efficiency of photosynthesis (Hill et al. 2005). Irradiance is thought to induce phosphorylation of the chlorophyll-protein complexes so that part of the complex moves across from PSII to 
PSI (Gorbunov et al. 2001). This component of NPQ has been shown to relax over a period of minutes upon darkening (Horton and Hague 1988, Hill et al. 2005). When ROS oxidises the thylakoid lipids, the membrane becomes less fluid affecting the ability for the chloropyll-protein complexes to rearrange and dissipate energy as heat. This is why different compositions of saturated and unsaturated lipids in the different Symbiodinium types affect their sensitivity to thermal and light stresses (Tchernov et al. 2004).

$\mathrm{qI}$, or photoinhibitory quenching, remains after dissipation of the $\Delta \mathrm{pH}(\mathrm{qE})$ and $\mathrm{qT}$, and normally becomes most active when light levels are in excess of that needed for photosynthesis (Baker et al. 2008). qI relaxes very slowly, over tens of minutes to hours (Hill et al. 2005). It is thought that this process is involved with inactivation of PSII, damage to PSII and quenching in the antenna pigments, as the carotenoids prevent activation of singlet oxygen in the chloroplast (Hill et al. 2005, Szabo et al. 2005)

Both partners in the symbiosis also use other protective mechanisms to tolerate stress. Both have pigments that may help in protection; algae have carotenoids and xanthins (Brown 1997), and the host has phytochromes, which lower the internal light environment, and chromoproteins that absorb photons before they reach the symbionts chloroplasts (Dove et al. 2008). Both partners can produce antioxidants to minimize the effects of oxidative damage on cellular components such as SOD and catalases (Lesser and Farrell 2004, Lesser 2006). There are several classes of SOD, some occurring only in the host and others only in the symbiont (Furla et al. 2005). Studies have found higher SOD activity in symbiotic anemones compared to aposymbiotic ones due to $\mathrm{O}_{2}$ produced from photosynthesis by the symbiont (Richier et al. 2005).

The host has mycosporine-like amino acids (MAAs) which act as a sunscreen against ultraviolet radiation, some of which may originate from the symbiont, translocated with the photosynthate (Shick and Dunlap 2002, Furla et al. 2005). Shick and Dunlap (2002) found that free-living species of dinoflagellates produce a wide variety of MAAs compared to those in symbiosis and hypothesised that this is due to the host taking on some of the protective role. The host can also rapidly synthesise heat-shock proteins, a type of molecular chaperone, in response to increased temperature (Fitt et al. 2001a, Csaszar Jr et al. 2009). Studies have found that freshly isolated Symbiodinium cells have 
a greater stress response than cultures of the same type, presumably because the host's protection has reduced the need for the algae to develop long-term photoacclimatory mechanisms (Abrego et al. 2008, Dove et al. 2008, Hennige et al. 2008).

\subsection{Tolerance and acclimation to environmental stress by change of}

\section{symbiont}

Bleaching events are increasing in frequency on a global scale (Hoegh-Guldberg et al. 2007, Hoegh-Guldberg and Bruno 2010), but during a bleaching event there is never total elimination of all corals from an entire reef suggesting that there is resistance to environmental stress in some partnerships (Mayfield and Gates 2007). One reason for tolerance may depend on the type of Symbiodinium being harboured at the time. Changes between specific host and symbiont combinations, and loss or uptake of symbionts have been proposed as mechanisms leading to tolerance and acclimation. For an adult coral to survive bleaching, it must either retain some of the population it already hosts or acquire symbionts from the environment (Coffroth et al. 2010). The "Adaptive Bleaching Hypothesis" ( $\mathrm{ABH}$ ) postulates that a change in environmental conditions will cause the loss of symbionts sensitive to present conditions and repopulation within the host of symbionts more tolerant to the conditions (Buddemeier and Fautin 1993, Berkelmans and van Oppen 2006). Tolerant symbionts can be changed or 'switched' with those from the outside environment or can be "shuffled' by increasing the relative abundance of 'cryptic' ones already present in the host that are better suited to the new environmental conditions (Baker 2001) (Figure1.10 ).

Studies have found that there has been possible switching from the environment in anemones (Kinzie et al. 2001) and soft corals (Lewis and Coffroth 2004) after bleaching. It was thought that switching was the most likely mechanism for a change in the symbiont population as, using conventional molecular methods, only $25 \%$ of corals had been found to associate with multiple types (Silverstein et al. 2012). 


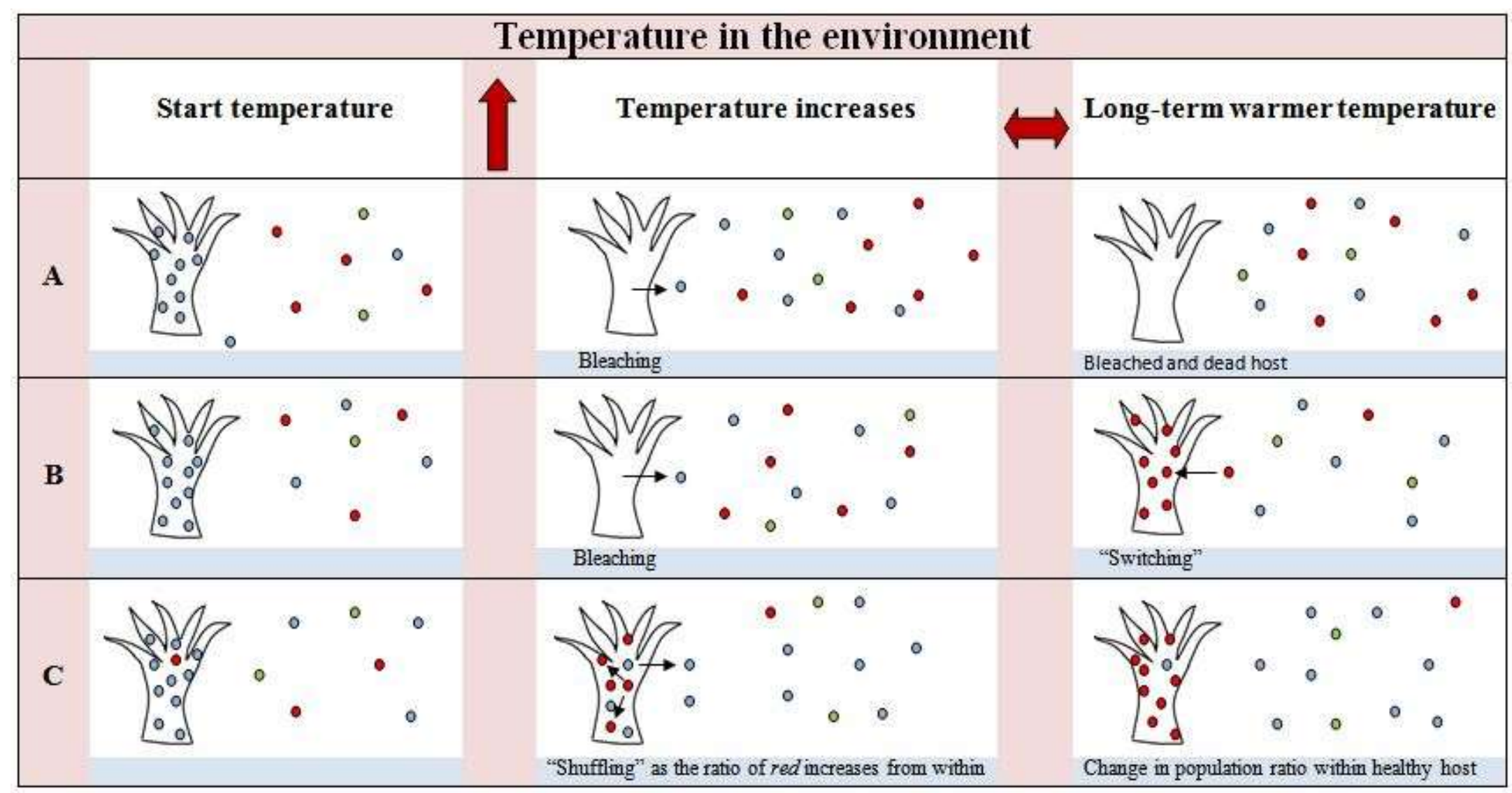

Figure 1.10: Diagrammatic representation of how host polyps react to changes in the environment, in this case an increase in temperature, by using 'switching' and 'shuffling' of symbionts from either the pool of Symbiodinium types from the surrounding water, or from within the host itself, to acclimate to the new conditions. In column 1, before temperature increases, polyps A and B host Symbiodinium blue, and polyp C hosts a larger population of Symbiodinium blue and a 'cryptic' population of Symbiodinium red. As the temperature increases (column 2), polyps A and B lose their symbionts in the process of bleaching, whereas polyp C remains healthy but the ratio between Symbiodiniums blue and red changes as "shuffling" occurs. As the environment remains at the new temperature, column 3, polyp A dies due to loss of its obligate symbiont, polyp B recovers but has now 'switched' symbionts from the environment to Symbiodinium red, and polyp C remains healthy but has 'shuffled' the population within it to having a larger population of Symbiodinium red and a 'cryptic' population of Symbiodinium blue. 
However, the advent of more sensitive, high-resolution, real-time PCR techniques, which can pick up very low abundances of symbionts in the host, has changed this point of view. A recent study found that at least one sample of all species tested (39 corals) hosted at least two clades of Symbiodinium and that $68 \%$ of all sampled colonies hosted at least two clades (Silverstein et al. 2012). This suggests that shuffling occurs more often than had been presumed, and also suggests that total bleaching, or loss of symbionts, does not have to occur for a differently structured population of symbionts in the host. However, when conditions return to normal for the area, many of the newly dominant types become competitively displaced by the original dominant symbionts (Lawler 2009, LaJeunesse et al. 2010).

The concept of an "ecospecies", or a single host that can function differently due to the Symbiodinium type it associates with, has evident advantages for living in a changing environment (Buddemeier and Fautin 1993, Berkelmans and van Oppen 2006). In temperate regions, where conditions do change cyclically, flexibility in the Symbiodinium partnership would explain how cnidarian hosts might be able to have populations with such wide latitudinal distribution, and live in habitats where temperature and irradiance can be so varied.

\subsection{Temperate $v s$ tropical}

Symbiotic relations between marine invertebrates and endosymbiotic dinoflagellates are abundant in oligotrophic tropical seas, where phototrophy, and conservation and recycling of essential nutrients are crucial for the survival of the host. The success of cnidarian-dinoflagellate symbiosis is such that the reefs dominate the shallow waters of the tropics with over 700 species of symbiotic corals found (Yellowlees et al. 2008). Coral reefs are found at lower latitudes, generally between $25^{\circ} \mathrm{N}$ and $25^{\circ} \mathrm{S}$ where mean winter sea surface temperatures (SST) do not fall below $18{ }^{\circ} \mathrm{C}$ (Savage et al. 2002a), and light penetration and aragonite saturation levels are sufficient (McDonald et al. 2008). In contrast, symbiotic relationships between invertebrates and symbiotic dinoflagellates are relatively uncommon in temperate seas (Muller-Parker and Davy 2001). Environmental conditions become more variable at higher latitudes, with seasonal changes in light, temperature and nutrient levels. However, even though fewer 
cnidarian species form symbioses in temperate regions, symbiotic anemones are locally abundant and ecologically important (Muller-Parker and Davy 2001).

Inheritance of endosymbionts in temperate regions is commonly through vertical or closed systems passed on by the mother, possibly due to the rarity of potential donors of Symbiodinium cells and of viable cells in the water column (Davy and Turner 2003). In the tropics, Symbiodinium cells are more readily available either because symbiotic invertebrates regularly release them into the water or because viable cells are present in the faeces of corallivores (Muller-Parker 1984). Horizontal or open systems are therefore more common in the tropics. The density of endosymbiont cells is far less in temperate associations than in the tropics, where there may be several million per square centimetre of host tissue (LaJeunesse 2002). However, as mentioned previously, the partnership in tropical cnidarians is unstabilised by small fluctuations in environmental parameters whereas temperate symbioses are typified by stable populations, even when subjected to extreme conditions.

Temperatures at higher latitudes are generally lower than in the tropics but the differences in minimum and maximum temperature are pronounced between different seasons and even on a daily scale. Intertidal anemones in rockpools can be exposed to a change in temperature of $10{ }^{\circ} \mathrm{C}$ during a summer's day (Dingman 1998). The stability of temperate symbioses in such fluctuating thermal conditions suggests that temperate partnerships are more tolerant of temperature change than are tropical ones, which have been shown to bleach with only minor changes in temperature and live close to their thermal limits (Brown 1997, Fitt et al. 2001a, Dunn et al. 2004).

For symbiotic cnidarians, light is an important environmental parameter as it determines the amount of photosynthetic carbon fixed by the symbionts. Levels of photosynthetically active radiation (PAR) are similar between the tropics and temperate regions during the summer, but during winter levels, at high latitudes, drop significantly in temperate regions (Muller-Parker and Davy 2001). Due to lower densities of Symbiodinium cells in temperate hosts and reduced light, the host receives lower quantities of organic carbon from its symbionts in the winter (Verde and McCloskey 2007) and may rely more on heterotrophic sources of organic carbon from nutrients in the water (Davy et al. 1996). Tropical cnidarians, on the other hand, rely heavily on 
their dinoflagellate symbionts, and would perish without the organic carbon and recycled nitrogen that they obtain from them (Smith and Douglas 1987, Wang and Douglas 1998, Furla et al. 2005).

Tropical waters are clear due to the lack of phytoplankton, and therefore zooplankton. However, tropical cnidarians may receive more than $100 \%$ of their energy requirements from their symbionts under well-lit conditions (Davies 1991, Falkowski et al. 1993). Temperate waters in contrast, are often turbid and nutrient rich. In temperate regions there are seasonal fluctuations in zooplankton, a food source for anemones, and consequently temperate anemones have a peak in both nutrient supplies, photosynthate from symbionts and zooplankton during summer (Muller-Parker and Davy 2001). Temperate anemones are able to rely solely on heterotrophic feeding (Davy et al. 1997b).

There have been few studies on temperate Symbiodinium diversity compared to those in the tropics, but they indicate it to be relatively low within hosts or among host species (LaJeunesse and Trench 2000, Savage et al. 2002a, Chen and Yang 2005, Focioli et al. 2011). For example there are only five anthozoans out of eighty around the United Kingdom that contain Symbiodinium algae (Davy et al. 1996). Symbiotic anemones can be found throughout temperate regions from $60^{\circ} \mathrm{N}$ in Alaska to $46^{\circ} \mathrm{S}$ in New Zealand (Buddemeier and Fautin 1996, Phillips 2006). This study will be on a small intertidal anemone, Anthopleura aureoradiata and the molecular identity and photophysiology of the Symbiodinium within.

\subsection{The study organism -Anthopleura aureoradiata}

Anthopleura aureoradiata is a small, brown coloured, symbiotic anemone, endemic to New Zealand. It was first described in 1909 from specimens found in Oriental Bay, Wellington and originally named Bunodes aureoradiata (Stuckey 1909). Stuckey gave a very full description of the anemone and noted the abundance of symbiotic dinoflagellates that could be seen in the endoderm. It is approximately $5-12 \mathrm{~mm}$ in diameter and is taller than it is wide, with 48 tentacles arranged in 4 circular rows. $A$. aureoradiata reproduces asexually and broods the young within its body cavity before they are expelled. The young are already in symbiosis with Symbiodinium cells when 
released (J. Howe, per.obs), suggesting that they have inherited the algae from their mother.

The anemone can be found abundantly on rocky shores or on mudflats from the far north at Cape Reinga to the far south on Stewart Island (Phillips 2006). Amongst tidal pools it is more commonly found in cracks and crevices above the water rather than within pools (J. Howe, pers. obs). On the mudflats it is commonly found attached to the shell of the cockle Austrovenus stutchbury, which are often buried in the sediment at low tide. When the cockles migrate up through the substrate, the anemones open up their tentacles along the surface. This enables the anemone to feed heterotrophically but also allows its symbionts to photosynthesise (Figure 1.11).

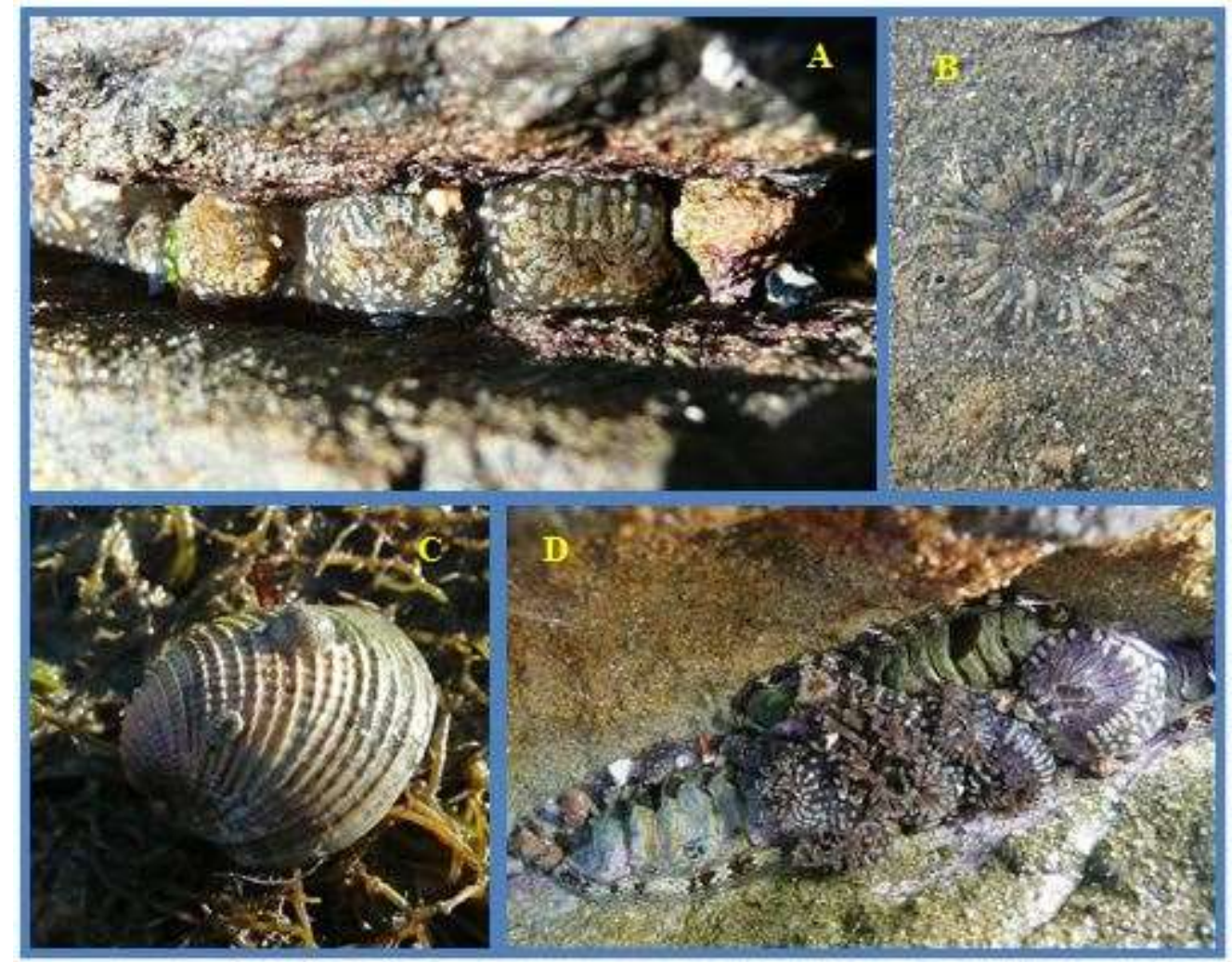

Figure 1.11: Anthopleura aureoradiata, which are approximately 5-12 $\mathrm{mm}$ in diameter. A) inside a crack on the rocks at Point Halswell, Wellington; B) extending its tentacles on the surface of the mudflat at Pauatahanui; C) attached to the cockle Austrovenus stutchburyi; and D) in a pool at Point Halswell. 


\section{$\underline{1.8 \text { Aims and objectives }}$}

A. aureoradiata was chosen as a model species as it has a symbiotic partnership with Symbiodinium cells. Unlike corals which bleach, or lose their symbionts with small changes in their environmental conditions, this anemone appears to be resilient to the extreme environmental conditions encountered in NZ. Of particular note, it survives the very cold winters in the far south and hot summer conditions in the far north without any evidence of bleaching. My research aim was to determine why and how this symbiotic relationship is so resilient.

The specific objectives were:

Objective 1 - To determine the molecular identity of the Symbiodinium spp. within the anemone, throughout its latitudinal range, and through the seasons, and whether seasonal changes differed in two habitats, the rocky shore and mudflats.

Hypothesis - That Symbiodinium diversity would be low but that there would be variance with latitude and season.

Objective 2 - To compare the molecular identity of the Symbiodinium spp. in New Zealand with those from four species of anemones from Europe (Cereus pedunculatus, Anthopleura ballii and Anemonia viridis from the south-west of England, and Aiptasia mutabilis from Brittany)

Hypothesis - The Symbiodinium spp. in New Zealand would be endemic, due to the isolation of these islands from other landmasses

Objective 3 - To determine whether resilience to the extreme differences in temperature and light in New Zealand is attributed to the Symbiodinium photoprotective mechanisms.

Hypothesis - The Symbiodinium photoprotective mechanisms would effectively and efficiently protect the photosystem and photosynthetic yield would therefore be low. 
The two data chapters are structured as independent manuscripts. Consequently there is some overlap between these chapters and a degree of repetition, especially in their introductions. 


\section{Chapter 2}

\section{The biogeography of Symbiodinium spp. within the New Zealand endemic anemone, Anthopleura aureoradiata}

\section{$\underline{2.1 \text { Introduction }}$}

Photosynthetic dinoflagellates of the genus Symbiodinium form symbiotic relationships with many marine hosts, including species of the phyla Porifera, Mollusca, Foraminifera, Radiolaria and Cnidaria (Smith and Bernays 1991, Hackett et al. 2004). Symbiotic cnidarians include corals, anemones and jellyfish. The ecological success of the reef-building corals within the oligotrophic waters of the tropics is attributed to the nutritional interactions between host and symbiont, that support the coral's respiration, growth and reproduction (Trench 1971, Muscatine and Kaplan 1994). Cnidarian/dinoflagellate symbiosis becomes more uncommon with increasing latitude. At temperate latitudes, where days are seasonally shortened, irradiance is reduced from cloudy skies, sea temperatures fluctuate and are generally cooler, and waters are more turbid and nutrient rich, nutritional dependence on the symbiont is not essential for host survival, (Davy et al. 1996, Davy et al. 1997a, Muller-Parker and Davy 2001). Nevertheless, temperate symbiotic cnidarians (especially sea anemones) are locally abundant and ecologically important (Savage et al. 2002a) and are known to be distributed from $60{ }^{\circ} \mathrm{N}$ in Alaska to $47^{\circ} \mathrm{S}$ in New Zealand (Buddemeier and Fautin 1996, Phillips 2006; J. Howe. pers. obs)

\subsubsection{Diversity within the genus Symbiodinium}

Members of the genus Symbiodinium are morphologically similar but are genetically diverse. To date there have been 9 molecular clades identified by analysing nuclear and chloroplast ribosomal DNA; these are clade A through to I (Pochon and Gates 2010). Within these clades, over 400 distinct internal-transcribed spacer-2 (ITS2) types have been detected (LaJeunesse 2002, LaJeunesse et al. 2004, Silverstein et al. 2012), though whether types are separate species is not clear (Stat et al. 2011). Restriction fragment 
length polymorphism (RFLP) of PCR-amplified small subunit ribosomal RNA (SSU rRNA) was the first technique used to identify the differences between Symbiodinium spp. and place them in a cladal tree (Rowan and Powers 1991). These authors named and placed clade $\mathrm{A}$ at the base of the tree and found members of this clade to be as closely related to free-living dinoflagellates as to symbiotic Symbiodinium. Confusingly, the order of divergence of the clades over time cannot be assumed by the nomenclature given, as clade E probably originated directly after A, followed by $\mathrm{G}$, then $\mathrm{D}$, then $\mathrm{I}, \mathrm{B}$, F, and $\mathrm{H}$ and finally $\mathrm{C}$ (Pochon and Gates 2010).

\subsubsection{Molecular techniques used to identify diversity in the genus Symbiodinium}

The discovery of the magnitude of Symbiodinium diversity, distribution and behaviour has come about with the use of ever more subtle molecular techniques. These include restriction fragment length polymorphisms (RFLP) (eg Rowan and Powers 1991, Rowan and Knowlton 1995), direct sequencing (eg Loh et al. 2001), sequencing of bacterially cloned ribosomal DNA (rDNA) (eg Apprill and Gates 2007), quantitative real-time PCR (qPCR) (eg Mieog et al. 2007) and size-screening with denaturing gradient gel electrophoresis (DGGE) (eg LaJeunesse et al. 2004) and single-stranded conformation polymorphisms (SSCP) (eg Ulstrup and Van Oppen 2003). Sequence comparison of rapidly evolving genes, such as the large subunit of rDNA, the hypervariable regions of the chloroplast $23 \mathrm{~S}$ rDNA, the microsatellite flanking regions and internal transcribed spacer regions of the rDNA (ITS1 and ITS2), make the distinction of subcladal types possible (LaJeunesse 2001, Loh et al. 2001, RodriguezLanetty et al. 2001, Van Oppen et al. 2001, Santos and Coffroth 2003, Chen et al. 2005, Hunter et al. 2007, Sampayo et al. 2007).

The ITS2 region is part of the eukaryotic nuclear rDNA operon and lies between the 5.8S and the 28S rRNAs (Schultz et al. 2006, Hunter et al. 2007). The organization of the operon is similar among all eukaryotes but the ITS2 region is highly diverse between species. This makes it a very useful marker for low level phylogenetic analysis (Hunter et al. 1997). However, while the sequences of this region are variable amongst species, the secondary structures formed, when single strands fold back on themselves, are well conserved across eukaryotes and can also be used to identify species. 


\subsubsection{The secondary structure as an identification tool}

The secondary structure has a similar four-fingered or four-helix shape across such divergent species as vertebrates and yeast, and green algae and higher plants (reviewed by Schultz et al. 2006) with the third helix being the longest. As the structural shape is a general feature across eukaryotes, it can be used as a way to differentiate the slower evolutionary time over which some clades diverged compared to the quicker divergence of one species from another (Schultz et al. 2006).

The secondary structure of Symbiodinium ITS2 takes one of two configurations. The more ancestral Symbiodinium clades and free-living dinoflagellates have the common 'four-fingered' configuration whereas those clades that diverged in more recent times have a sub-helix (III a), making it a 'five-fingered' structure (Gottschling and Plotner 2004) (Figure 2.1).

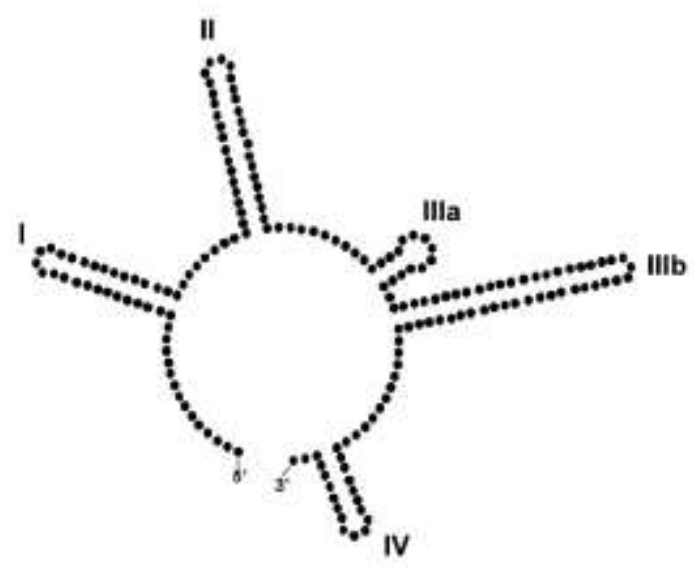

Figure 2.1: Representation of a Symbiodinium secondary structure with five helices. Figure taken from Hunter et al. (2007).

Analyses by Hunter et al. (2007) have found that clades A, E, G and D have four helices and clades B, C, F and H have five (Figure 2.2). Pochon et al. (2010) later identified another clade and named it clade I, placing it one branch above clade D and as a sister to the group including clades $\mathrm{F}, \mathrm{B}, \mathrm{H}$ and $\mathrm{C}$. They also found that the secondary structure of the ITS2 from clade I folded into a five-helix configuration. 


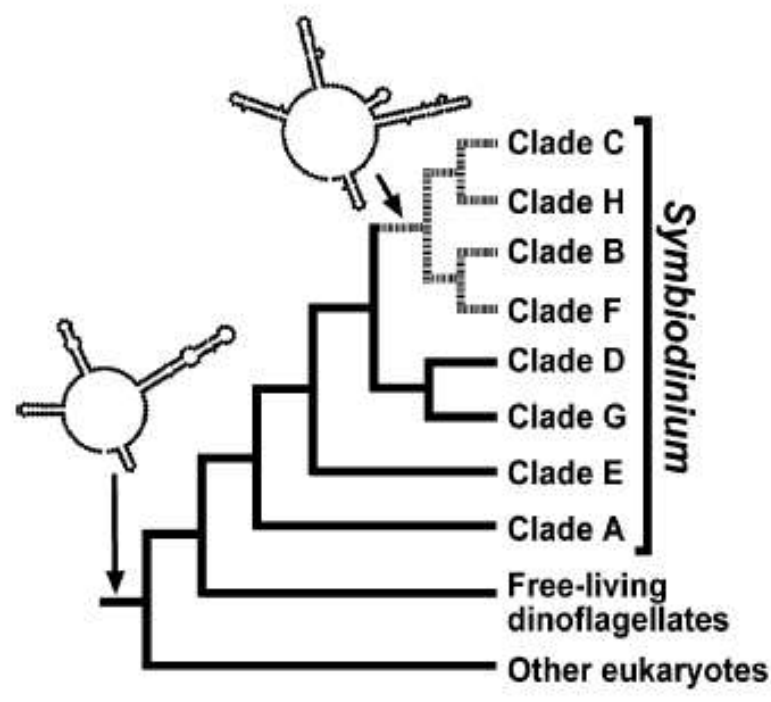

Figure 2.2: The two variations of secondary structures of Symbiodinium ITS2 and its relationship with the cladal phylogeny. The phylogenetic consensus cladogram comes from various studies (see Coffroth and Santos 2005). Figure comes from Hunter et al. (2007).

The study by Hunter et al. (2007) also found that sequences of base pairs (bp) at the bases of helix I and helix II show variability between Symbiodinium types but are conserved within a type, giving further support of the usage of secondary structures as an identification tool.

\subsubsection{Distribution of the genus Symbiodinium}

The diversity and distribution of Symbiodinium clades and types varies with geographical and latitudinal location, habitat type and depth. There is evidence of scleractinian corals from the Eastern Pacific hosting mainly clade $\mathrm{C}$ whereas in the Caribbean they host mainly clade B with some A and C (LaJeunesse et al. 2003). To the west of the Pacific, $\mathrm{C}$ is still the dominant clade with A, B and D in less than $15 \%$ of corals (LaJeunesse et al. 2003). On the reefs off Eilat (Red Sea), only A and C have been found (Karako-Lambert et al. 2004) and in the NE Atlantic and Mediterranean Sea, most Symbiodinium are of clade A (Savage et al. 2002a, Visram et al. 2006). Different phylotypes are thought to have distinctive physiological traits in response to different light and temperature conditions (Baker 2001). Consequently, as temperature and irradiance are the environmental factors that vary most with latitude, they are believed to have an influence on Symbiodinium distribution. For instance, clades that are present at high latitudes are probably better adapted physiologically to function with 
the seasonally changing conditions (Rodriguez-Lanetty et al. 2001, McDonald et al. 2008).

Due to the apparent partitioning of cladal distribution within a habitat, studies have been carried out that suggest that genetically distinct Symbiodinium possess unique environmentally adaptive capabilities (Rowan 2004). Clade A algae are thought to be shallow-water specialists (LaJeunesse 2002) as they may have a competitive edge over other clades by producing mycosporine-like amino acids, thereby giving protection to their hosts and their own cells from UV light (Banaszak et al. 2000). They have also been characterized as being 'invasive' and 'opportunistic' (Rowan 1998, Toller et al. 2001, Stat et al. 2008b). Clade B algae have also been described as 'sun specialists', while clade $\mathrm{C}$ algae have been proposed as 'shade specialists', and E and D as 'stress tolerant' (Rowan 1998, Toller et al. 2001, Savage et al. 2002b). However, other studies have found that associating one clade with certain traits is too restrictive as there is variation among types within a clade (Iglesias-Prieto and Trench 1994, Tchernov et al. 2004, Hennige et al. 2008). Moreover, particular host-symbiont combinations also influence the position of the holobiont in the environment (Sampayo et al. 2007, Wicks et al. 2010b).

The 'ecospecies' concept proposes that a host species will function differently depending on the symbionts it associates with, and thereby can adapt to changing conditions (Buddemeier et al. 2004). Some cnidarian species are thought to react to changes in the environment by changing the type of symbionts within. The changes would function as an adaptive mechanism, as suggested by the 'Adaptive Bleaching Hypothesis' (Buddemeier and Fautin 1993), where changing environmental conditions trigger the loss of intolerant types from the host which are then 'switched' for more tolerant symbionts from the water column (Baker 2001, Berkelmans and van Oppen 2006). Full bleaching, or loss of symbionts, might not have to occur for a change of the Symbiodinium type present (Chen et al. 2005, Venn et al. 2008b) and new symbionts need not come from the external environment. Instead, a 'shuffling' of the Symbiodinium community within the host can occur as the relative abundance of one type over another ensues as competitive dominance changes (Baker 2001). Shuffling is thought to occur more often than once thought. The majority of coral colonies on the 
Great Barrier Reef have been found to harbour multiple symbiont types, one dominant with a background level of other types (Berkelmans and van Oppen 2006, Mieog et al. 2007). Shuffling has been shown to occur with season (Chen et al. 2005), with latitude (LaJeunesse and Trench 2000) and habitat, and after natural bleaching events (Thornhill et al. 2006).

Some host species with long latitudinal distributions have been found to change symbionts from one type to another, often with both types present along a midway point. Anthopleura elegantissima, from the Washington and Oregon coastlines, hosts clade B (Symbiodinium muscatinei) which then becomes replaced by clade E (Symbiodinium californium) in Southern California (LaJeunesse and Trench 2000). Latitudinal patterns also occur in the corals Plesiastrea versipora and Acropora millepora along the eastern Australian coast (Rodriguez-Lanetty et al. 2001, Berkelmans and van Oppen 2006) and in Oulastrea crispate in its distribution from Thailand to Japan (Lien et al. 2007). Differences in habitat as the depth changes on the same reef have also been correlated to changes in symbionts. The Caribbean coral Monastraea annularis (sensu lato) has a change in Symbiodinium clade which is depth related (Rowan and Knowlton 1995) as does the Bermudan anemone Condylactis gigantea (Venn et al. 2008b). This anemone was also found to undergo a change in symbiont clade with the seasons (Venn et al. 2008b). More extraordinary are the anemones Anthopleura elegantissima and Anthopleura xanthogrammica, as these anemones may not only contain the two Symbiodinium endosymbionts mentioned above, but also another algal taxon, a symbiotic chlorophyte or 'zoochlorella'. Light and, to a greater extent, temperature, affect the ratio of the three symbionts within $A$. elegantissima. Higher latitudes, lower shore positions and cooler seasons favour $S$. muscatinei and zoochlorellae whereas lower latitude, higher shore positions and warmer seasons favour S. californium (Bates 2000, LaJeunesse and Trench 2000, Secord and Augustine 2000, Muller-Parker et al. 2007, Verde and McCloskey 2007).

There have been comparatively fewer studies of temperate than tropical Symbiodinium diversity. Most of these temperate studies have found a relatively low symbiont diversity, whether within a host species or among host species (LaJeunesse and Trench 2000, Savage et al. 2002a, Chen et al. 2005, Visram et al. 2006). Some suggest that 
there is a trend for endemicity in high latitude Symbiodinium types (Savage et al. 2002a, Wicks et al. 2010b) and host specificity due to the strong selection pressure imposed on the symbionts by seasonally variable environmental conditions (Wicks et al. 2010b, Focioli et al. 2011).

\subsubsection{The temperate anemone, Anthopleura aureoradiata}

Anthopleura aureoradiata is a small, brown-coloured endemic anemone from New Zealand (Figure 2.3). It largely reproduces asexually and broods its young within its body cavity. The young are released harbouring Symbiodinium cells (J. Howe. pers. obs), suggesting that they inherit their dinoflagellates vertically from their mother. The anemones are found throughout NZ ranging from $34^{\circ} \mathrm{S}$ to $47^{\circ} \mathrm{S}$ (Phillips 2006) and can be found on the rocky shore and on mudflats.

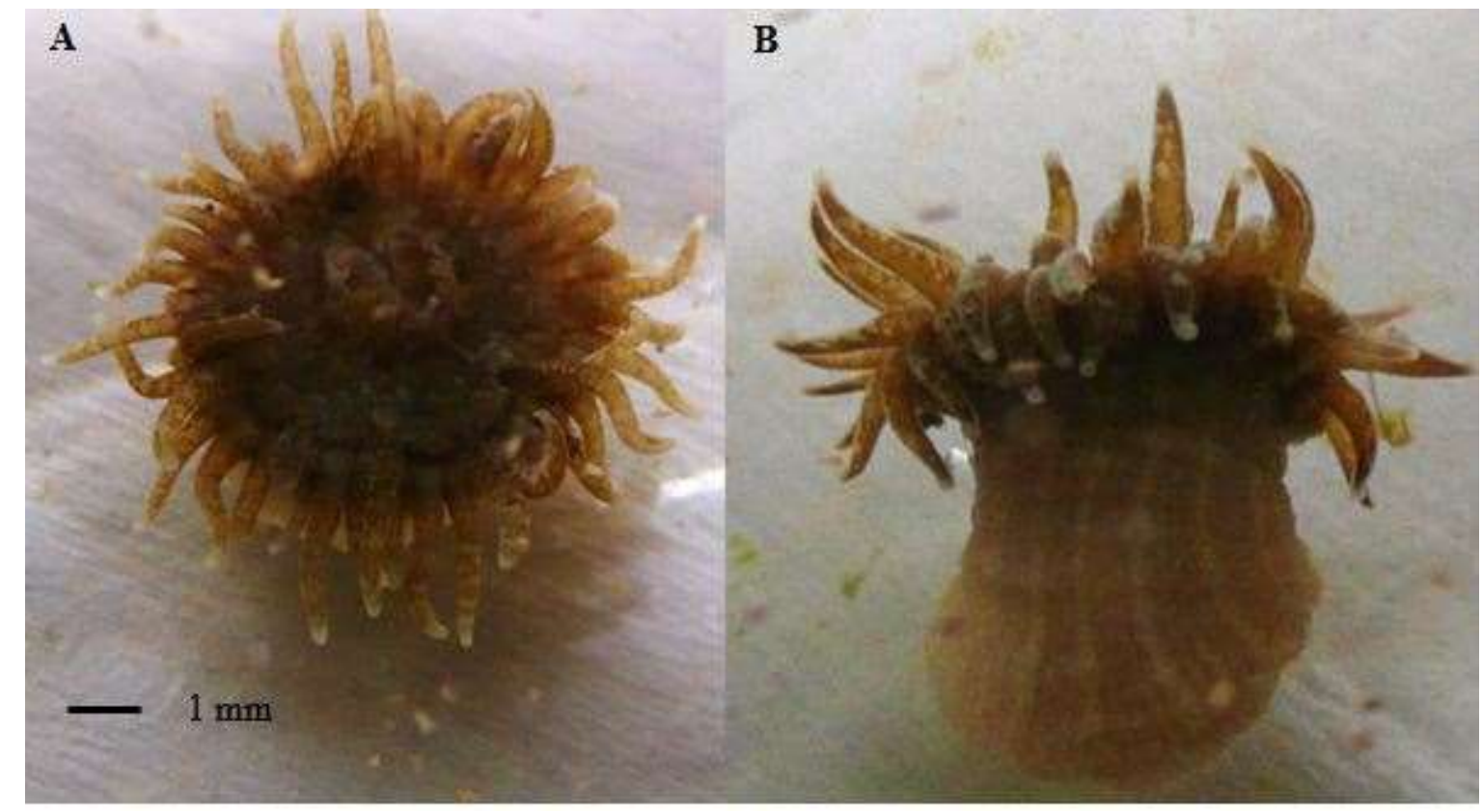

Figure 2.3: Anthopleura aureoradiata as seen from (A) above and from (B) the side displaying its characteristic brown tentacles and thick column.

\subsubsection{Aims and Objectives}

The purpose of this study was to determine the molecular identity of the Symbiodinium cells within A. aureoradiata by sequencing and identifying the ITS2 region of the algae, using PCR and SSCP techniques. The objectives were: 
1. To determine if there is a change in symbiont identity and/or population composition with latitudinal gradient within anemones throughout the length of NZ.

2. To determine if there is a change in symbiont identity and/or population composition through the seasons within anemones. A preliminary study was made to determine whether the variability is also correlated to habitat type (mudflat versus rocky shore).

3. To compare the genetic identities of the Symbiodinium cells in NZ anemones to four species of anemones from Europe (Cereus pedunculatus, Anthopleura ballii and Anemonia viridis from the south-west of England and Aiptasia mutabilis from Brittany, (France) to identify differences or similarities between Symbiodinium originating from a similar latitudinal distance away from the Equator This comparison was based on the hypothesis that temperate Symbiodinium diversity is low and possibly closely related, due to the difficulties of living in extreme conditions.

The predictions for the New Zealand study were that the Symbiodinium diversity would be low, but that there may be genetic variation with latitude and season due to adaptation to the differences in climate with time and distance. The prediction was also that there would be endemicity in the Symbiodinium type as a result of NZ being isolated from other landmasses.

\section{$\underline{\text { 2.2 Materials and Methods }}$}

\subsubsection{Study Area and Sampling}

The anemones collected for the latitudinal study came from five sites throughout New Zealand during the early southern autumn in March 2011 (Table 2.1). These were, from south to north, Stewart Island, Dunedin, Pauatahanui Inlet near Wellington, Raglan and Parengarenga Harbour (Figure 2.4). All sites were mudflats at the mouth of estuaries and the anemones collected were attached to Austrovenus stutchburyi cockles, which were shallow-buried in the sandy sediment. 
Figure 2.4: Collection sites around New Zealand for the latitudinal molecular study. (Photograph of Raglan by Emma Gibbin).
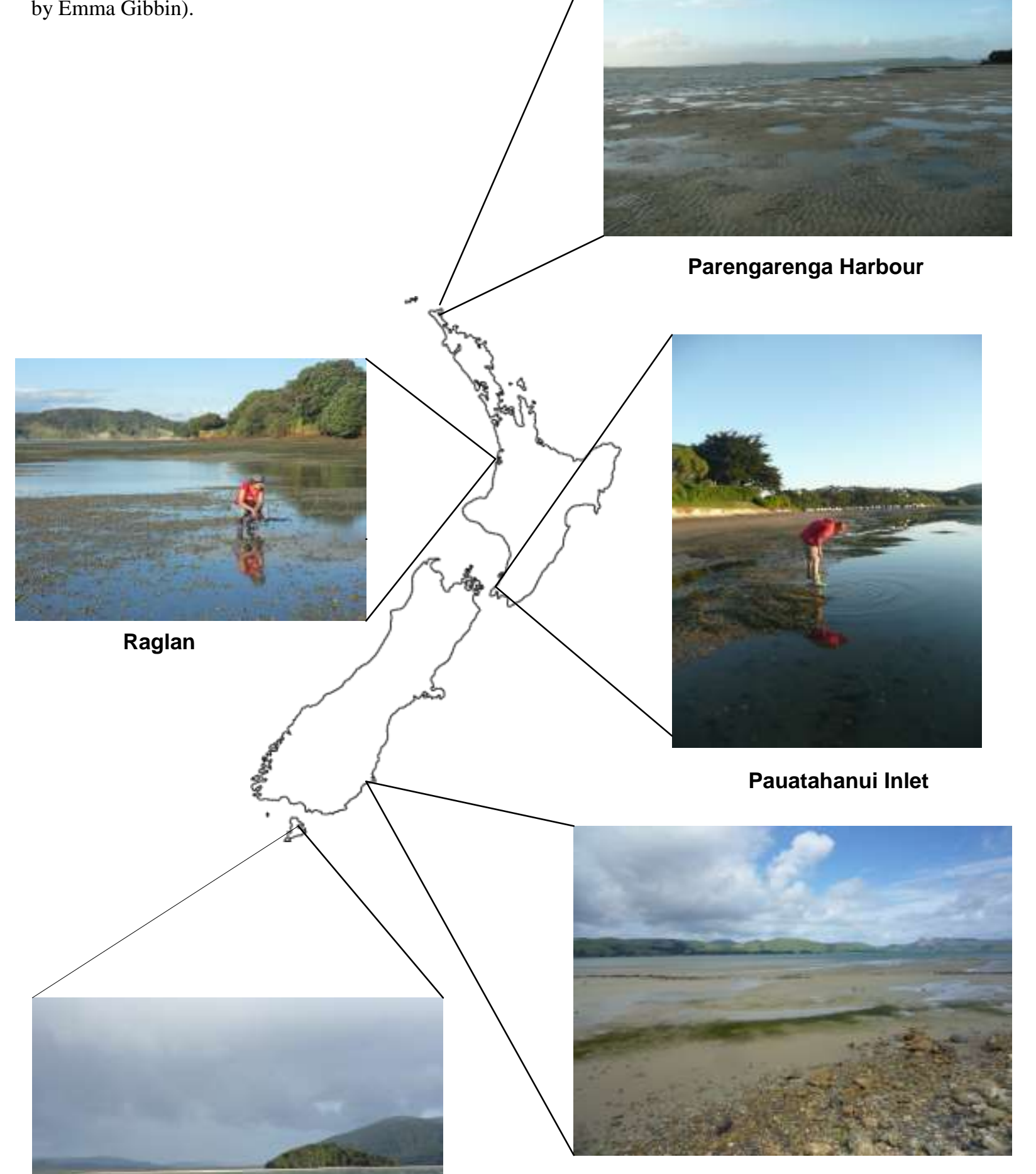

Aramoana, Dunedin

Mudflat Island, Stewart Island 
The anemones for the temporal and habitat study were collected either from the mudflats at Pauatahanui or from the rocky shore at Point Halswell, Kau Bay, within Wellington Harbour (Figure 2.5). The anemones from Kau Bay were collected from the midlittoral zone, between 1-5 $\mathrm{m}$ above the low tide mark. The tidal range in Wellington is small, being $2 \mathrm{~m}$ or less, with 2 tides per day (Land Information New Zealand (LINZ)). Four anemones were collected in a $10 \mathrm{~m}^{2}$ area before moving along a transect parallel to the shore for $10 \mathrm{~m}$, to reduce the chances of collecting clonal anemones, before another four anemones were collected in the same sized area. These sites were visited once in the middle of each season (mid-April, midJuly and mid-January) for the year beginning in the autumn 2011 and finishing in the summer 2012 (Table 2.1). 


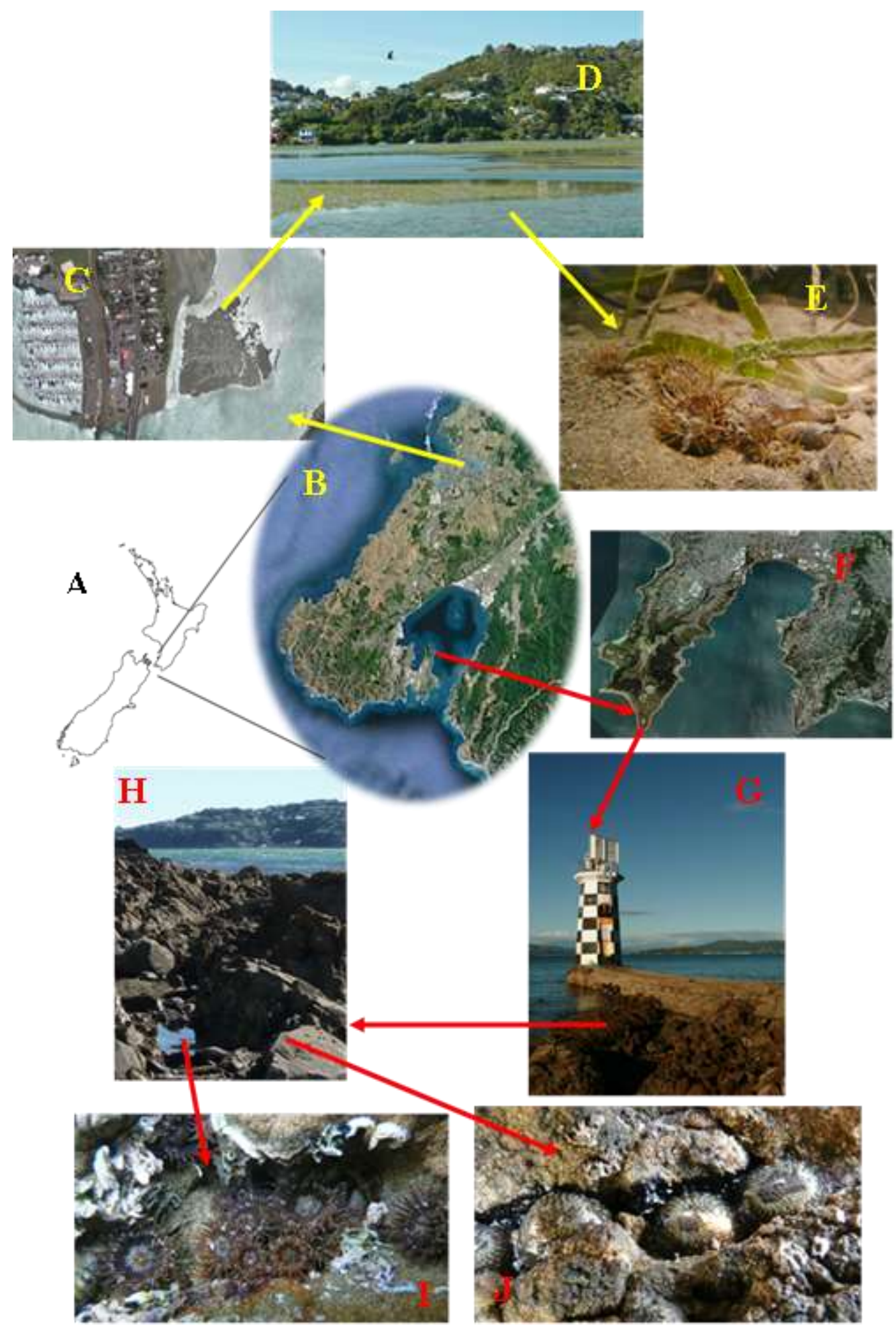

Figure 2.5: Collection sites and habitats around Wellington. A) map of NZ; B) Close-up of the southern end of the North Island; C) and D) Collection site at Pauatahanui Inlet; E) Anthopleura aureradiata on the cockle Austrovenus stutchburyi, within a seagrass bed; F) Kau Bay in Wellington Harbour; G) The lighthouse on Point Halswell in Kau Bay; $\mathbf{H})$ The rocky shore to the west of the lighthouse where the rocky shore collection was made. Anthopleura aureoradiata within I) shallow tidal pool or $\mathbf{J}$ ) a rocky crevice. B), C) and $\mathbf{H}$ ) are from Google Earth. 
Table 2.1 - Collection data for all anemones analysed in this study giving species, site collected, coordinates for the site and the date collected.

\begin{tabular}{|c|c|c|c|}
\hline Anemone Collected & Collection Site & Coordinates & Date Collected \\
\hline \multicolumn{4}{|l|}{ For Latitudinal Study } \\
\hline \multirow[t]{5}{*}{ Anthopleura aureoradiata } & Stewart Island & $46^{\circ} 54^{\prime} 346^{\prime \prime} \mathrm{S}, 168^{\circ} 00^{\prime} 589^{\prime \prime} \mathrm{E}$ & $4^{\text {th }}$ March 2011 \\
\hline & Dunedin & $45^{\circ} 46^{\prime} 60^{\prime \prime} \mathrm{S}, 170^{\circ} 41^{\prime} 60^{\prime \prime} \mathrm{E}$ & $6^{\text {th }}$ March 2011 \\
\hline & Pauatahanui Inlet, Wellington & $41^{\circ} 6^{\prime} 4.85^{\prime \prime} \mathrm{S}, 174^{\circ} 52^{\prime} 17^{\prime} \mathrm{E}$ & $10^{\text {th }}$ March 2011 \\
\hline & Raglan & $37^{\circ} 47^{\prime} 52^{\prime \prime} \mathrm{S}, 174^{\circ} 42^{\prime} 36^{\prime \prime} \mathrm{E}$ & $22^{\text {nd }}$ March 2011 \\
\hline & Parengarenga Harbour & $34^{\circ} 32^{\prime} 5^{\prime \prime} \mathrm{S}, 172^{\circ} 56^{\prime} 43^{\prime \prime} \mathrm{E}$ & $24^{\text {th }}$ March 2011 \\
\hline For Temporal Study & & & $\underline{\text { Autumn }}$ \\
\hline \multirow[t]{8}{*}{ Anthopleura aureoradiata } & Pauatahanui Inlet, Wellington & $41^{\circ} 6^{\prime} 4.85^{\prime}, \mathrm{S}, 174^{\circ} 52^{\prime} 17^{\prime \prime} \mathrm{E}$ & $14^{\text {th }}$ April 2011 \\
\hline & Kau Bay, Wellington & $41^{\circ} 17^{\prime} 2.42^{\prime \prime} \mathrm{S}, 174^{\circ} 49^{\prime} 33.89^{\prime} \mathrm{E}$ & $15^{\text {th }}$ April 2011 \\
\hline & & & Winter \\
\hline & Pauatahanui Inlet, Wellington & & $13^{\text {th }}$ July 2011 \\
\hline & Kau Bay, Wellington & & $14^{\text {th }}$ July 2011 \\
\hline & & & $\underline{\text { Summer }}$ \\
\hline & Pauatahanui Inlet, Wellington & & $18^{\text {th }}$ January 2012 \\
\hline & Kau Bay, Wellington & & $17^{\text {th }}$ January 2012 \\
\hline \multicolumn{4}{|l|}{$\underline{\text { European Collection }}$} \\
\hline Anthopleura ballii & Private aquarium (originated, Scilly Isles, England) & $49^{\circ} 93^{\prime} 63^{\prime \prime} \mathrm{N}, 6^{\circ} 3^{\prime} 23.0^{\prime \prime} \mathrm{W}$ & December 2010 \\
\hline \multirow[t]{2}{*}{ Anemonia viridis } & West Hoe, Plymouth, England & $50^{\circ} 21^{\prime} 49^{\prime \prime} \mathrm{N}, 4^{\circ} 8^{\prime} 42.15^{\prime \prime} \mathrm{W}$ & $14^{\text {th }}$ December 2010 \\
\hline & Firestone Bay, Plymouth & $50^{\circ} 21^{\prime} 33.59^{\prime \prime} \mathrm{N}, 4^{\circ} 9.57^{\prime} 64^{\prime \prime} \mathrm{W}$ & $2^{\text {nd }}$ December 2010 \\
\hline Cereus pedunculatus & West Hoe, Plymouth & $50^{\circ} 21^{\prime} 49^{\prime} \mathrm{N}, 4^{\circ} 8^{\prime} 42.15^{\prime} \mathrm{W}$ & $17^{\text {th }}$ December 2010 \\
\hline Aiptasia mutabilis & Roscoff, Brittany, France & $48^{\circ} 43^{\prime} 38.46^{\prime \prime} \mathrm{N}, 3^{\circ} 59^{\prime} 17.68^{\prime \prime} \mathrm{W}$ & $5^{\text {th }}$ May 2011 \\
\hline
\end{tabular}




\subsubsection{Climatic conditions in New Zealand}

The mean seasonal temperatures in $\mathrm{NZ}$ range from $19{ }^{\circ} \mathrm{C}$ in the summer to $6.5^{\circ} \mathrm{C}$ in the winter, though the lowest and the highest temperatures range from $-4{ }^{\circ} \mathrm{C}$ in the south to $34{ }^{\circ} \mathrm{C}$ in the north. The mean daily minimum and maximum temperatures also has a wide range with latitude (Table 2.2). The UV radiation from the sun is intense with more sunlight reaching the surface around New Zealand in the spring and summer than autumn and winter. (More detailed climate data for New Zealand, compiled from daily local measurements from NIWA's National Climate Database (Cliflo Retrieved 6 June 2012), are provided in Appendix 1).

Table 2.2: Mean daily minimum and maximum temperature of the 5 years prior to the latitudinal collection of anemones throughout New Zealand. Three weather stations nearest to collection sites were chosen. Tiwai Point is the furthest south in New Zealand, Paraparaumu Aerodrome is in the middle and Cape Reinga is the furthest north. Data collected from the National Climate Database from NIWA (Cliflo Retrieved 6 June 2012)

\begin{tabular}{|c|c|c|c|}
\hline Weather station site & Season & $\begin{array}{c}\text { Mean daily } \\
\text { minimum temperature }\end{array}$ & $\begin{array}{c}\text { Mean daily } \\
\text { maximum temperature }\end{array}$ \\
\hline \multirow[t]{2}{*}{ Tiwai Point, Southland } & Winter & $3.31{ }^{\circ} \mathrm{C}$ & $9.82{ }^{\circ} \mathrm{C}$ \\
\hline & Summer & $10.54{ }^{\circ} \mathrm{C}$ & $17.12^{\circ} \mathrm{C}$ \\
\hline \multirow[t]{2}{*}{ Paraparaumu Aerodrome } & Winter & $5.45^{\circ} \mathrm{C}$ & $12.84{ }^{\circ} \mathrm{C}$ \\
\hline & Summer & $13.14^{\circ} \mathrm{C}$ & $20.70^{\circ} \mathrm{C}$ \\
\hline \multirow[t]{2}{*}{ Cape Reinga, Northland } & Winter & $10.83^{\circ} \mathrm{C}$ & $15.06^{\circ} \mathrm{C}$ \\
\hline & Summer & $16.06^{\circ} \mathrm{C}$ & $21.83^{\circ} \mathrm{C}$ \\
\hline
\end{tabular}




\subsubsection{European anemone collection}

The anemones Cereus pedunculatus and Anemonia viridis from England were collected by SCUBA from the south-western coastline around Plymouth. The English anemone, Anthopleura ballii, had been held in an aquarium and was provided by Dr Keith Hiscock (Marine Biological Associaton of the UK). Aiptasia mutabilis was collected by SCUBA from the northern shore off Roscoff, Brittany and was provided by Station Biologique de Roscoff. (see Table 2.1 for collection details)

Each A. aureoradiata collected in New Zealand was placed immediately into a $15-\mathrm{ml}$ tube containing approximately $14 \mathrm{ml}$ of $99 \%$ DNA grade ethanol. This ethanol was changed the following day and the tubes then stored at $-20{ }^{\circ} \mathrm{C}$. Anemones from Europe were stored in 99\% DNA grade ethanol for shipment to New Zealand. On arrival, the ethanol was changed and the tubes stored at $-20{ }^{\circ} \mathrm{C}$.

\subsubsection{DNA extraction and Symbiodinium spp. identification}

Symbiodinium cells were isolated by homogenizing a small piece of tissue from each anemone. The tissue was ground in individual 1.5-ml Eppendorf collection tubes using a tissue grinder attached to a Proxxon Micropower Driver in $200 \mu 1$ extraction buffer (1 $\mu \mathrm{M}$ filtered seawater (FSW), $5 \mathrm{mM}$ EDTA and $0.025 \%$ Triton X-100). The slurry was centrifuged at $3000 \mathrm{x}$ g for 5 minutes and the supernatant discarded. The remaining pellet was re-suspended in $500 \mu \mathrm{l}$ extraction buffer (FSW and $5 \mathrm{mM}$ EDTA), centrifuged (3000 x g for 5 minutes) and the supernatant discarded again. This final stage was repeated another 3 times. An aliquot $(200 \mu \mathrm{l})$ of guanidinium buffer $(25 \mathrm{ml}$ guanidinium isothtiocyante $4 \mathrm{M}, 0.1514 \mathrm{~g}$ Tris $50 \mathrm{mM}, 0.09306 \mathrm{~g}$ EDTA $10 \mathrm{mM}$; heated to $65{ }^{\circ} \mathrm{C} ; 0.5152 \mathrm{~g}$ Sarkosyl $70 \mathrm{mM}, 0.25 \mu \mathrm{l} \beta$-mercaptoethanol) was then pipetted into the tube and the contents vortexed and stored at $-4^{\circ} \mathrm{C}$ for a week for DNA extraction (Stat et al. 2009). The tubes were then centrifuged, "short spin", to collect all the liquid at the bottom of the tube and heated to $72{ }^{\circ} \mathrm{C}$ for 10 minutes, during which time they were vortexed every 2-3 minutes, before being centrifuged again (16000 x g for 5 minutes). Supernatant $(100 \mu \mathrm{l})$ was transferred to new tubes and $100 \mu \mathrm{l}$ isopropanol added to each. These tubes were stored overnight at $-20{ }^{\circ} \mathrm{C}$. The next day the tubes were centrifuged ( $16000 \mathrm{x}$ g for 15 minutes), the supernatant removed, and the 
tubes and DNA extract then "washed" in $100 \mu 170 \%$ ethanol and centrifuged once more (16000 x g for 10 minutes). Supernatant was again removed and the tubes and extracted DNA dried under a laminar-flow hood for an hour to remove all traces of ethanol. Tris $0.1 \mathrm{M} \mathrm{pH} 8(50 \mu \mathrm{l})$ was added to each tube. These tubes were placed onto ice and vortexed every 15 minutes for an hour. The DNA samples were stored at $-20{ }^{\circ} \mathrm{C}$, ready for genotyping.

Table 2.3: Identity and codes given for anemones that were processed and sequenced. The last column has the number of Symbiodinium sequences that were good enough to be used to assemble phylogenetic trees.

\begin{tabular}{|c|c|c|c|}
\hline Anemone name/place or habitat collected & $\begin{array}{l}\text { Coding used to } \\
\text { identify samples } \\
\text { analysed }\end{array}$ & $\begin{array}{l}\text { Number } \\
\text { processed }\end{array}$ & $\begin{array}{l}\text { Number used for } \\
\text { tree construction }\end{array}$ \\
\hline \multicolumn{4}{|c|}{ European Study: } \\
\hline Anthopleura ballii & $\mathbf{A b}$ & 3 & 2 \\
\hline Anemonia viridis & Av & 4 & 4 \\
\hline Cereus pedunculatus & $\mathbf{C}$ & 5 & 5 \\
\hline Aiptasia mutabilis & $\mathbf{A M}$ & 10 & 10 \\
\hline \multicolumn{4}{|c|}{ Latitudinal Study: Anthopleura aureoradiata } \\
\hline Stewart Island & $\mathbf{S}$ & 6 & 6 \\
\hline Dunedin & $\mathbf{D}$ & 5 & 5 \\
\hline Pauatahanui, Wellington & $\mathbf{W}$ & 5 & 3 \\
\hline Raglan & $\mathbf{R}$ & 5 & 2 \\
\hline Parengarenga Harbour & PH & 6 & 4 \\
\hline \multicolumn{4}{|c|}{ Temporal Study: Anthopleura aureoradiata } \\
\hline Kau Bay, Autumn & $\mathbf{K A}$ & 22 & 13 \\
\hline Pauatahanui, Autumn & PA & 5 & 1 \\
\hline Kau Bay, Winter & $\mathbf{K W}$ & 11 & 9 \\
\hline Pauatahauni, Winter & $\mathbf{P W}$ & 3 & 0 \\
\hline Kau Bay, Summer & $\mathbf{K U}$ & 6 & 4 \\
\hline Pauatahanui, Summer & PU & 2 & 1 \\
\hline
\end{tabular}


Not all anemones collected were used for molecular identification; rather, enough were used to generate as rigorous a dataset as possible within the timeframe available. The numbers processed and those that provided good enough sequences to use for phylogenetic tree constructions are given in Table 2.3.

Symbiodinium types were characterised using the ITS2 region of the nuclear ribosomal DNA with the following Symbiodinium-specific ITS2 primers: forward primer (ITsD), 5'-GTG AAT TGC AGA ACT CCG TG-3'; reverse primer (ITS2rev2), 5'-CCT CCG CTT ACT TAT ATG CTT-3'(Pochon et al. 2007). PCR reactions were performed using MyTaq $^{\mathrm{TM}}$ Mix (Bioline, Australia) in a total volume of $30 \mu \mathrm{l}(1 \mu \mathrm{l}$ DNA template, $13 \mu \mathrm{l}$ dd $\mathrm{H}_{2} \mathrm{O}, 15 \mu \mathrm{MyTaq}{ }^{\mathrm{TM}} \mathrm{Mix}$ and $0.5 \mu \mathrm{l}$ of each primer $10 \mathrm{mM}$.) The amplification profile was adapted from the MyTaq TM Mix protocol and consisted of 3 minutes $95{ }^{\circ} \mathrm{C}$ (initial denaturation), 29 cycles of 15 seconds $95{ }^{\circ} \mathrm{C}$ (denaturation), 15 seconds $60{ }^{\circ} \mathrm{C}$ (annealing) and 15 seconds $72{ }^{\circ} \mathrm{C}$ (extension) with a final holding temperature of $4{ }^{\circ} \mathrm{C}$. The PCR was run on a Veriti ${ }^{\mathrm{TM}}$ Thermal Cycler (PN4375786) machine.

The ITS2 amplified PCR products were run for 2 hours 20 minutes at 100 volts on a SSCP system with a vertically run polyacrylamide gel $(1.5 \mathrm{ml}$ polyacrylamide $(40 \%$

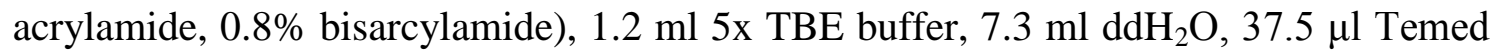

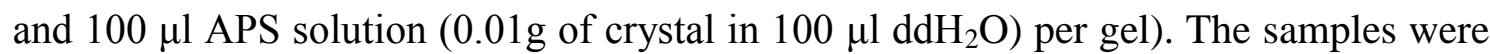
first denatured by heating $8 \mu \mathrm{l}$ of PCR-amplified DNA with $8 \mu \mathrm{l}$ of formamide loading dye per tube $(25 \mathrm{mM}$ EDTA pH 8 with $50 \mathrm{mg} / \mathrm{ml}$ of blue dye, diluted with $100 \%$ formamide 4:1 ratio (Sambrock et al. 1989)) for 5 minutes at $95{ }^{\circ} \mathrm{C}$, followed by snapcooling for 10 minutes on ice. This allowed the two separated DNA strands to fold back onto themselves into their secondary structure. Along with the denatured PCR product, a double-stranded DNA sample was also run as a reference standard to help with band interpretation as not all the DNA in the denatured product would have split and folded.

To identify distinct Symbiodinium types, prominent bands were excised with a pipette tip under UV light. The tips were placed in 1.5-ml tubes with $20 \mu \mathrm{lof} \mathrm{ddH}_{2} \mathrm{O}$ and swirled overnight on ice using a table-top rotary-shaker to dissolve the DNA. These samples were reamplified using the same amplification profile as before using a total volume of $30 \mu \mathrm{l}$ ( $5 \mu \mathrm{l}$ of dissolved DNA template, $9.5 \mu \mathrm{ddH}_{2} 0,15 \mu \mathrm{l} \mathrm{\textrm {MTaq } ^ { \mathrm { TM } }}{ }^{\mathrm{Mix}}$ 
and $0.25 \mu \mathrm{l}$ of each primer $10 \mathrm{mM}$ ). The PCR product was re-run on another SSCP gel to check band positions in relation to the position of the excised band from the previous gel.

Results of a SSCP gel rely on the size and shape of the secondary-structure fold of a denatured single-stranded DNA (Sunnucks et al. 2000, Sampayo et al. 2009). If there is only one type of Symbiodinium in the sample, then there should be two bands in the gel from the two halves of the DNA strands. Different size and shape folded structures will terminate at different distances along the gel. Differences of bases along a sequence, even just one base, can change the configuration of the fold and therefore affect the migration dynamics of the DNA strand. If there was number of types within a sample, even of the smallest proportion, it could result in the multiple bands. Therefore, four banded lanes would mark a sample that contained two Symbiodinium types or type variations and eight banded lanes, four types or type variations. The standard procedure for identifying types using a SSCP gel is that once a two band patterned sample has been sequenced and identified, all future samples that have the same band pattern, and have the same terminal position, can be inferred to be the same type. Early on in this study it was noted that samples sent for sequencing that had the same two-banded SSCP gel pattern were being identified as being similar or identical to two different Symbiodinium types. Therefore all samples were sent for sequencing and identity was not inferred by comparing SSCP gel band patterns.

Successful PCR amplifications were prepared for sequencing by cleaning 16-25 $\mu 1$ of the product with $1 \mu \mathrm{l}$ of ExoSAP-IT ${ }^{\mathrm{TM}}$ (Exonuclease I and Shrimp Alkaline Phosphatase in buffer - USB Corporation, Australia). This was incubated for 30 minutes at $37{ }^{\circ} \mathrm{C}$ in the PCR machine and then at $80{ }^{\circ} \mathrm{C}$ for a further 15 minutes. The samples were sequenced in both directions at Macrogen Inc., Korea.

Chromatograms were checked and aligned and overhangs removed using the software Geneious Pro 5.5.7 (Drummond et al. 2011). Retrieved sequences were identified using the Basic Local Alignment Search Tool (BLAST) for nucleotide sequences (blastn) of the National Center for Biotechnology Information (NCBI) BLAST database. A search was also carried out in the ITS2 sequence database (GeoSymbio) from the Gates Laboratory at the Hawaii Institute of Marine Biology (HIMB) (Franklin et al. 2012). 
Additional ITS2 sequences for the analyses of phylogenetic relatedness of the Symbiodinium types in this study were retrieved from GeoSymbio and NCBI. These included the free-living dinoflagellate Gymnodinium simplex (GenBank accession no: JN558104.1) and Symbiodinium clade A members (A1, A1.1, A1.2, A2, A3, A4, A5, A6, A7, A8, A9, A10, A11, A12, A13, A15, A16; GenBank accession no: AF333505, AF333504, AB207210, AF333506, DQ838546, AF333509, AF333508, AY686646, AY239360, AY258468, FJ224014, EU792882, FJ224013, EU792883, AF333504, EU792884 and EU792888).

\subsubsection{Data analysis}

\subsubsection{Phylogenetic tree construction}

Phylogenetic analyses were performed in Geneious Pro 5.6.2 (Drummond et al. 2011) using the plugins, PhyML v 2.0.12 (Vincent Lefort and Biomatters Ltd) (Guindon and Gascuel 2003) and MrBayes v 2.0.3 (Marc Suchard and Biomatters Ltd) (Huelsenbeck and Ronquist 2001). The Maximum Likelihood (ML) method tree topology was determined using a bootstrap analysis (1000 replicates) to infer tree topology robustness with PhyML. A GTR substitution model (discrete gamma), with four substitution rate categories and program values for all other parameters were chosen. The initial tree was obtained via BioNJ (Gascuel 1997). The GTR model specifications were: Likelihood: Loglk $=-1275.61322$, proportion of invariable sites: 0.000 , relative rate parameters: $\mathrm{A}$ $\leftrightarrow \mathrm{C}: 1.47498, \mathrm{~A} \leftrightarrow \mathrm{G}: 2.82482, \mathrm{~A} \leftrightarrow \mathrm{T}: 1.54658, \mathrm{C} \leftrightarrow \mathrm{G}: 2.16469, \mathrm{C} \leftrightarrow \mathrm{T}: 3.27153, \mathrm{G}$ $\leftrightarrow \mathrm{T}: 1.00000$.

A bayesian tree reconstruction with posterior probabilities was inferred using MrBayes v 2.0.3 (Marc Suchard and Biomatters Ltd) (Huelsenbeck and Ronquist 2001) using the same datasets. This tree was rooted using Gymnodinium simplex as the outgroup. Four Markov chains were run for 2,000,000 generations with trees sampled every 400 generations, with 100,000 initial trees discarded as "burn-in".

\subsubsection{Secondary structure}

ITS2 secondary structures were analysed after being folded by default on Geneious. This application did not use the templates set by Hunter et al. (2007). This meant that 
neither the four-helix nor the five-helix configurations were made. However, the Geneious fold made the equivalent of Helix I and Helix II so that the sequences of base pairs at the root of the helices could be compared to the secondary structures from the 2007 study.

The 3' end of the base on Helix I of all ITS2 sequences within members of Symbiodinium clade A from Hunter et al's (2007) study have the base sequence variability of GAGCAG (Types A1, A2 A3 and A4), GGGCAG (Symbiodinium sp. Amed), and GGGTGG ('Cryptic A) (see Appendix A2.4 for diagrammatic representations of secondary structures of clade A Symbiodinium).

The 3' end of the base on Helix II of all ITS2 sequences within members of Symbiodinium clade A from Hunter et al.'s (2007) study have the base sequence variability of ACTGGCATGCTA (Types A1, A2, A3 and A4), ACTGGCAGCGT (Symbiodinium sp. Amed), ATTGGCATGCTC ('Cryptic A').

The next six bases then read AATATC (Type A1), AGATTT (Symbiodinium sp. Amed), AGTACC (A3), AGTATC (Type A4), AGTATT ('Cryptic A') and AATATC (Type A2).

\subsection{Results}

\subsubsection{PCR and SSCP Gels}

Positive PCR bands were approximately in-line with the position of 350 base pairs as shown by the Hyperladder II. This is what was expected for Symbiodinium ITS2 amplifications. When the amplified products were run on SSCP gels, a range of band patterns were found (Figure 2.6). Winter and summer samples from the temporal/habitat study returned more than two bands. When more than two bands were present, and were excised and reamplified successfully, the resulting SSCP band patterns were the same (Figure 2.6 F) 


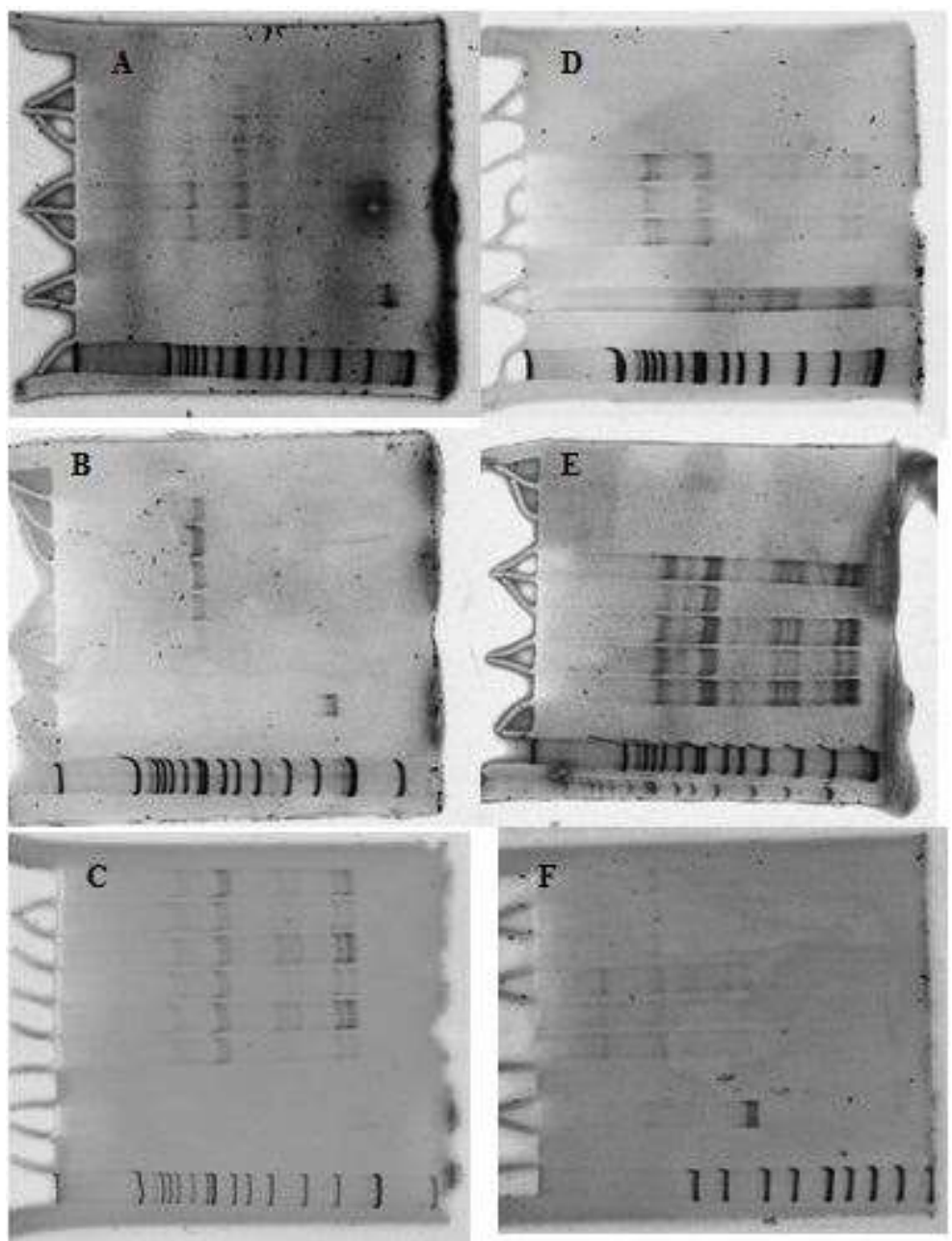

Figure 2.6: Photographs of stained SSCP gels showing the patterns returned from the amplified PCR products. Gels A) and B) have 2 bands; D) has 4 bands and C) and E) have 8 bands. Gel F) shows the band pattern on reamplifying the excised bands from earlier gels.

Migration of the single strand DNA relies on the temperature of the buffer surrounding the gel, and this relies on the temperature of the room the SSCP equipment is situated (Nataraj et al. 1999, Sunnucks et al. 2000). Each SSCP gel-run in this study usually had the same band patterns in all lanes run i.e. all the lanes had 2, 4 or 8 bands. If band patterns were due to samples' sequence variation, due to some anemones hosting more than one type, there should have been a variation of band patterns in the lanes for each gel-run. Therefore, thermal instability in the lab could have also led to some inconsistencies in the SSCP gel results. 


\subsubsection{Symbiodinium identity for all anemones in this study}

\subsubsection{Sequencing of all samples}

A total of 98 samples were sequenced (Table 2.3). The sequence BLAST searches identified all the study samples as being nearest to, or identical to, the ITS2 region in 'Symbiodinium sp. Mediterranean clade A' (Meron et al. 2012) (hereafter termed 'Med clade A') or 'Symbiodinium sp. Amed' (Hunter et al. 2007) (hereafter termed 'Amed') (Table 2.4 ) (see Appendix A2.2 and A2.3 for sequences). In other words, $A$. aureoradiata hosts two Symbiodinium types that are identical to or very closely related to those in anemones collected in England and in Brittany (France), and to Med clade A or Amed. Sixty-nine sequences were more than $95 \%$ pairwise identical to either of these types (Table 2.5), and were "tidy" enough to be used to construct the phylogenetic trees. The 'messiness' of the other sequences may have been the result of the host anemone containing both Symbiodinium types simultaneously.

Table 2.4: Details of Symbiodinium types suggested by NCBI Database and GeoSymbio BLAST searches as being most similar or identical to all samples identified in this study

\begin{tabular}{|lllll|}
\hline \multicolumn{1}{|c}{$\begin{array}{c}\text { Symbiodinium } \\
\text { species }\end{array}$} & \multicolumn{1}{c}{$\begin{array}{c}\text { Genbank } \\
\text { accession no. }\end{array}$} & Journal citation & \multicolumn{1}{c|}{$\begin{array}{c}\text { Location of } \\
\text { species source }\end{array}$} & \multicolumn{1}{c|}{ Host species } \\
Mediterranean & JN242191.1 & (Meron et al. & Ischia Island, Italy & Balanophyllia \\
clade A & & 2012) & & europaea \\
\hline Amed & DQ865210.1 & (Hunter et al. & Eastern & Anemonia sp. \\
& & 2007) & Mediterranean & \\
\hline
\end{tabular}

All specimens of $C$. pedunculatus and $75 \%$ of $A$. viridis from south-west England hosted Symbiodinium cells identical to Amed whereas all A. ballii and $90 \%$ of $A$. mutabilis from Brittany contained symbionts that were similar or identical to Med clade A (Table 2.5) $96.55 \%$ of the A. aureoradiata in the latitudinal study contained symbionts that were most similar to Med clade A; just one of these anemones from the far north of NZ contained symbionts that were more similar to Amed (Table 2.5). In the temporal study (Table 2.5), all winter-collected anemones from all the habitats hosted Symbiodinium cells that most closely matched to Med clade A. However, in summer 
and autumn there were some anemones that hosted symbionts that were more closely matched to Amed, (12.5\% and $22.22 \%$ of anemones respectively). The preliminary comparison of the two habitat types suggests that the presence of Amed with the seasonal change occurred at both sites (Table 2.6).

Table 2.5: Numbers of samples sequenced from the study identified by Geneious BLAST search as being most similar to, or identical to either Med clade A or Amed. The number of samples with more than $95 \%$ pairwise identical bases to these two types are also given. Only these sequences were used in the final molecular comparison of Symbiodinium types to make the phylogenetic trees. The sequences less than 95\% identical may have been 'messier' due to the sample anemone hosting both Symbiodinium types simultaneously.

\begin{tabular}{|c|c|c|c|c|c|c|}
\hline \multirow{2}{*}{ 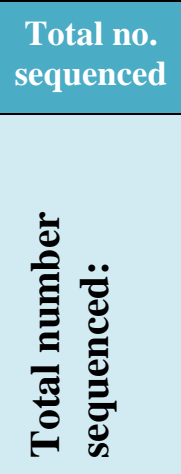 } & \multicolumn{3}{|c|}{$\begin{array}{c}\text { Number identified as Symbiodinium sp. } \\
\text { Mediterranean clade A }\end{array}$} & \multicolumn{3}{|c|}{$\begin{array}{l}\text { Number identiffed as } \\
\text { Symbiodinium sp. Amed }\end{array}$} \\
\hline & 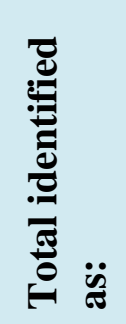 & 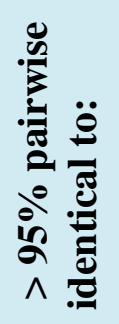 & 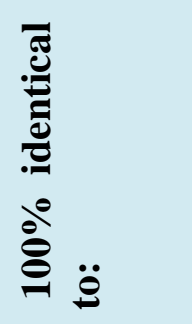 & 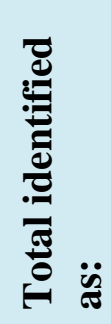 & 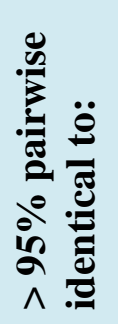 & 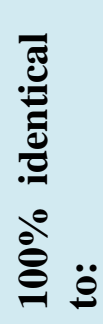 \\
\hline \multicolumn{7}{|c|}{$\begin{array}{l}\text { European study } \\
\text { Anthopleura ballii }\end{array}$} \\
\hline 3 & 3 & 2 & 0 & 0 & 0 & 0 \\
\hline \multicolumn{7}{|c|}{ Cereus pedunculatus } \\
\hline 5 & 0 & 0 & 0 & 5 & 5 & 5 \\
\hline \multicolumn{7}{|c|}{ Anemonia viridis } \\
\hline 4 & 0 & 0 & 0 & 4 & 4 & 3 \\
\hline \multicolumn{7}{|c|}{ Aiptasia mutabilis } \\
\hline 10 & 9 & 9 & 3 & 1 & 1 & 1 \\
\hline \multicolumn{7}{|c|}{ Latitudinal study } \\
\hline 27 & 26 & 20 & 4 & 1 & 0 & 0 \\
\hline \multicolumn{7}{|c|}{$\begin{array}{l}\text { Habitat study in } \\
\text { Autumn }\end{array}$} \\
\hline 27 & 21 & 11 & 0 & 6 & 3 & 0 \\
\hline \multicolumn{7}{|c|}{ Habitat study in Winter } \\
\hline 14 & 14 & 9 & 0 & 0 & 0 & 0 \\
\hline \multicolumn{7}{|c|}{$\begin{array}{l}\text { Habitat study in } \\
\text { Summer }\end{array}$} \\
\hline 8 & 7 & 5 & 1 & 1 & 0 & 0 \\
\hline
\end{tabular}


Table 2.6: Number of samples sequenced from each habitat in each season and the number of sequences identified as being clearly more similar, or identical, to either Symbiodinium sp. Med clade A or Symbiodinium sp. Amed.

\begin{tabular}{|r|c|c|}
\hline $\begin{array}{r}\text { Season, Symbiodinium } \\
\text { type and no. collected }\end{array}$ & Rocky shore & Mudflat \\
\hline Autumn $\mathbf{2 7}$ total & $\mathbf{2 2}$ & $\mathbf{5}$ \\
\hline Med clade A & 17 & 4 \\
\hline Amed & 5 & 1 \\
\hline Winter $\mathbf{- 1 4}$ total & $\mathbf{1 1}$ & $\mathbf{3}$ \\
\hline Med clade A & 11 & 3 \\
\hline Amed & 0 & 0 \\
\hline Summer $\mathbf{8}$ total & $\mathbf{6}$ & $\mathbf{2}$ \\
\hline Med clade A & 5 & 2 \\
\hline Amed & 1 & 0 \\
\hline
\end{tabular}

\subsubsection{Secondary structure}

The Geneious default secondary structure fold had the equivalent of Helix I and Helix II from the template configuration used by Hunter et al. (2007) and confirmed that the study samples were either identical to or very closely related to Med clade A or Amed (Figure 2.7)

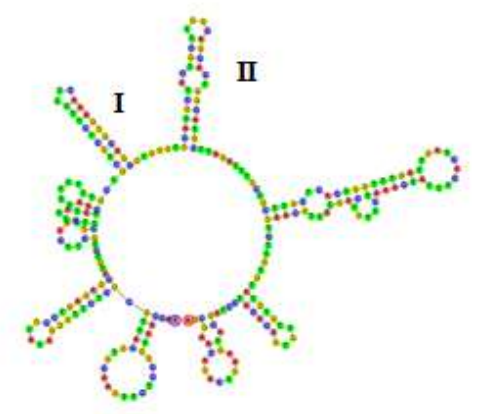

Figure 2.7: ITS2 sequence of D5 (sample from Dunedin) folded with the Geneious default folding function. Helices I and II are in the top and top left of the configuration. 
The 3' end of the base on Helix I of all the samples sequenced from the European and the New Zealand anemones in this study had the GGGCAG sequence which is the same as in the original fold of Symbiodinium sp. AMed by Hunter et al. (2007) (See Figure $2.8 \mathrm{~A})$.
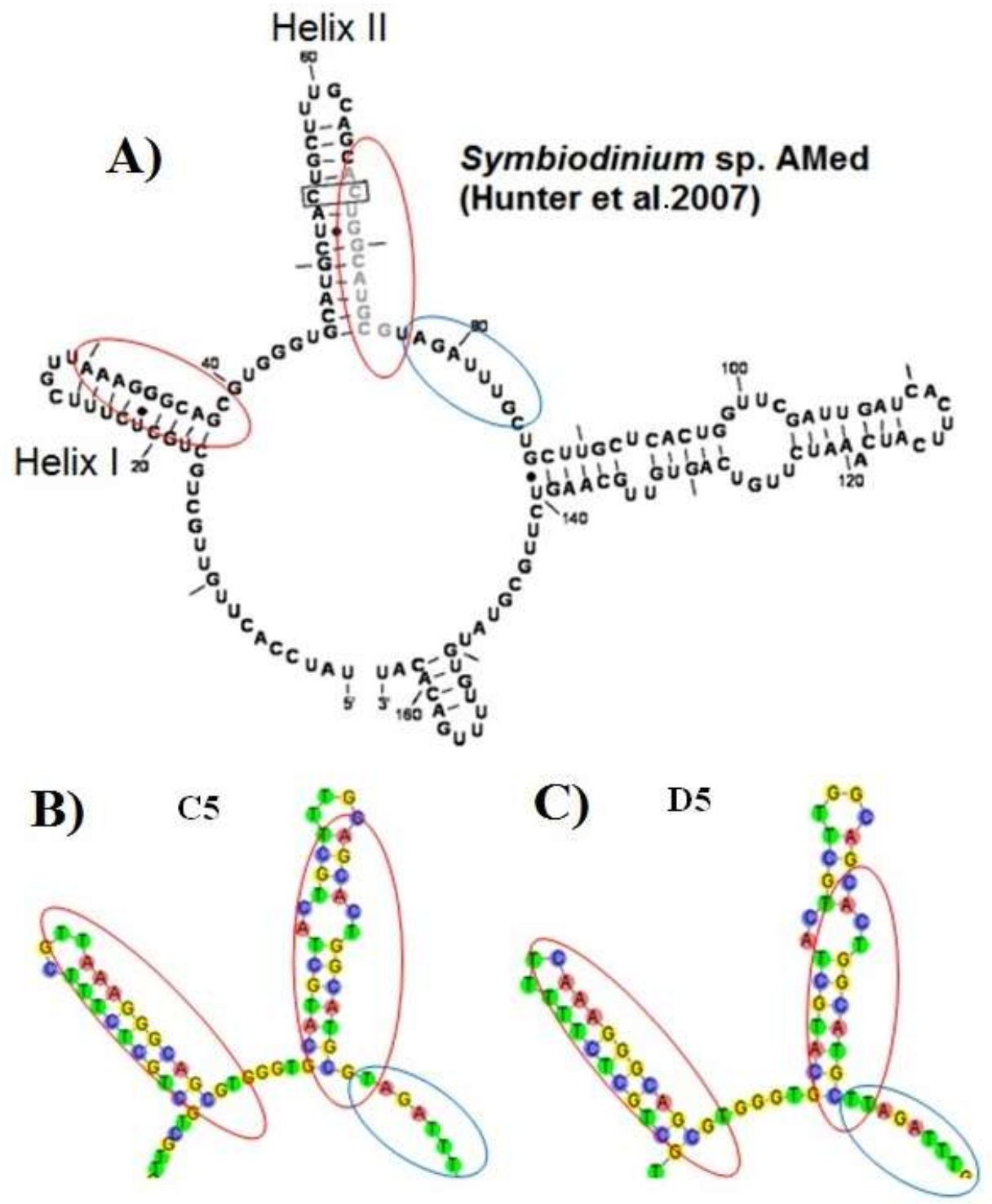

Figure 2.8: Secondary structures of ITS2 sequences for A) rRNA of Symbiodinium sp. Amed, which is why bases are labelled as "U" for uracil and not "T" for thymine; B) rDNA of C5 (C. pedunculatus); and C) rDNA of D5 (A. aureoradiata from Dunedin). Helix I in all three images has the same base sequences (as seen in the red ovals on the left of the three folds). Helix II, in Amed and C5, has the sequence ACTGGCATGCGT whereas Helix II in D5, which was more closely related to Med clade A, has the sequence ACTGGCATGCTT (as seen in the red ovals on the right of all three folds). All three then continue from the base of Helix II with the sequence AGATTT (as seen in the blue oval). 
The 3' end of the base on Helix II of all the samples in this study that were identified as being most similar to Symbiodinium sp. AMed had the sequence ACTGGCATGCGT (Figure $2.8 \mathrm{~A}$ ), the same as the original fold of Symbiodinium sp. AMed by Hunter et al. (2007). This is illustrated by the secondary structure fold of C5 (Figure 2.8 B). However, all the samples sequenced in this study that were identified as being most similar to Med clade A had the sequence ACTGGCATGC $\underline{T}$ T. This is illustrated by the secondary structure fold of D5 (Figure 2.8C). Then, in the original Symbiodinium sp. AMed fold (Hunter et al. 2007), and in the folds from all the samples in this study, the bases sequence read towards the 3' end with the sequence AGATTT (Figure 2.8), which is different to all other clade A secondary structure folds (see Appendix A2.3).

\subsubsection{Phylogenetic analyses}

The genetic relatedness of the Symbiodinium types identified in this study to other members of clade A, Gymnodinium simplex, Symbiodinium sp. Amed and Symbiodinium Mediterranean clade A was carried out using the Geneious programme. Before a tree was constructed, all the sequences being analysed were aligned and ends trimmed so that the sequences were mostly the length of the ITS2 sequence of Med clade A (260 bases). The resulting phylogenetic trees constructed under Maximum Likelihood (ML) (Figure 2.9) and Bayesian criteria (Figure 2.10) produced similar topologies. 


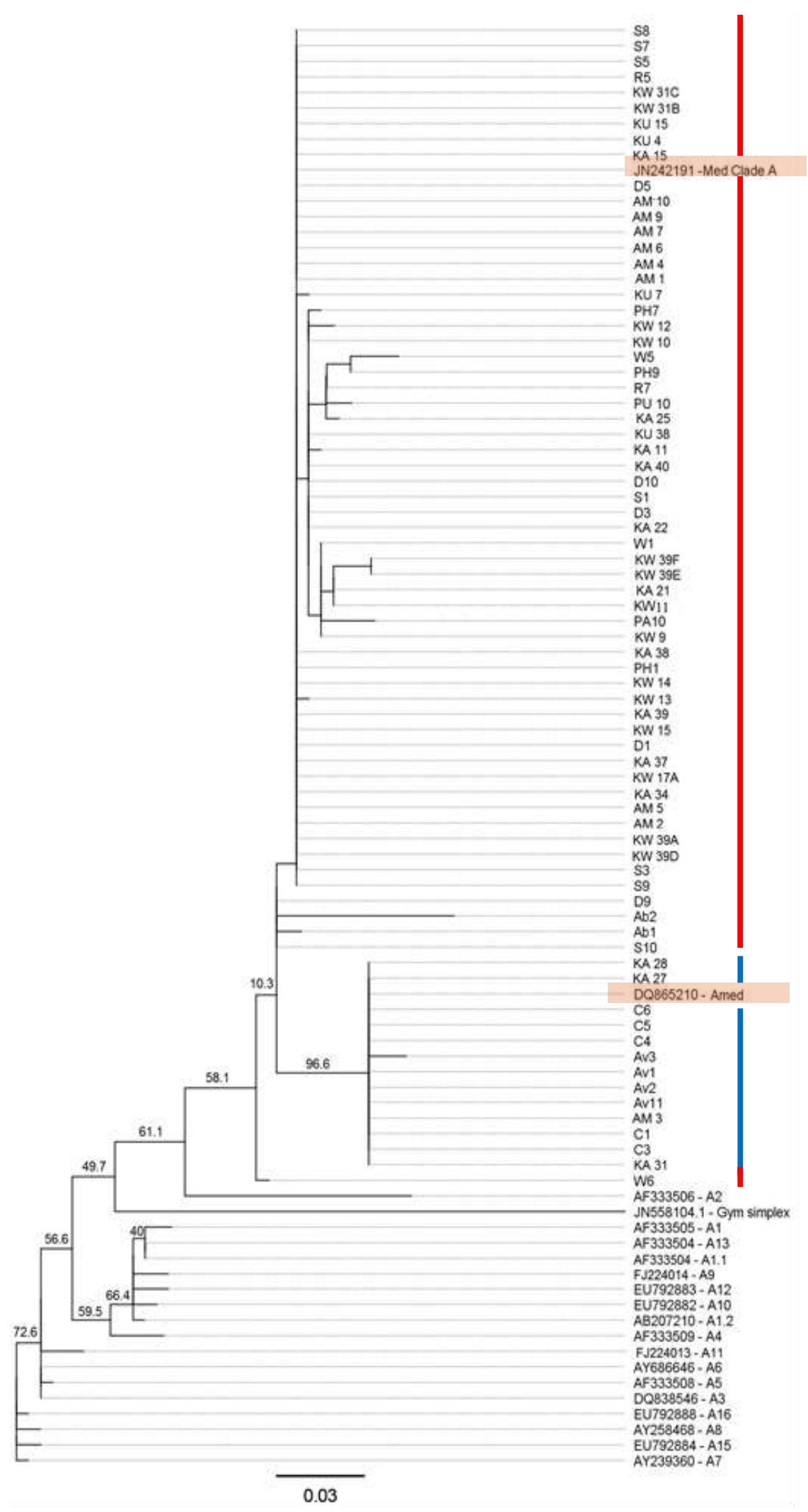

Figure 2.9: Maximum-likelihood tree of sampled Symbiodinium types and reference types inferred from ITS2 amplification, using Gymnodinium simplex as the outgroup. Taxa codes for the reference types were obtained from GenBank. Other labels indicate sample codes (see Table 2.3). Numbers correspond to bootstrap values (> 40.0) for 1000 replicates obtained with ML analysis (except at the node showing division of the Amed group from the Med clade A group (bootstrap value 10.3). (_ samples identified with Med Clade A; __ samples identified with Amed and; highlights Med Clade A and Amed sequences from Genbank). 


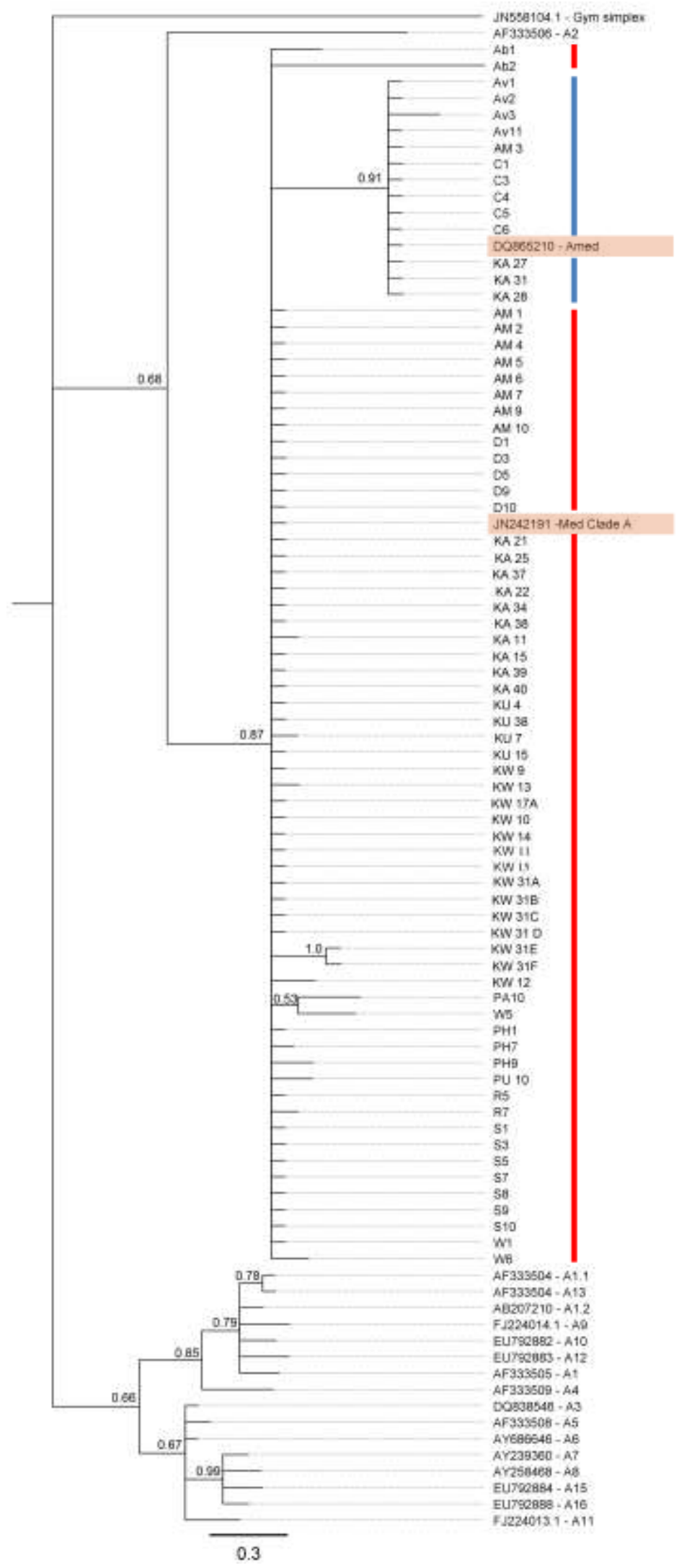

Figure 2.10: Bayesian tree of sampled Symbiodinium types and reference types inferred from ITS2 amplification.. This tree was rooted using Gymnodinium simplex as the outgroup. Taxa codes for the reference types were obtained from GenBank. Other labels indicate sample codes (see Table 2.3). Numbers correspond to posterior probabilities (> 0.50) inferred using MrBayes. samples identified with Med Clade A; samples identified with Amed and; highlights Med Clade A and Amed sequences from Genbank). 
Both trees placed Symbiodinium type A2 as a sister group to the Med clade A and Amed groups (bootstrap value: 59.0, posterior probability 0.68). The ML tree placed the Symbiodinium Amed group as a distinct group within the Symbiodinium Med clade A group (bootstrap value: 96.6) (Figure 2.9). The bootstrap value in this case means that in $96.6 \%$ of the bootstrap replications (1000 replicates in this analysis), the Amed group was placed as a group within the Med clade A group. The Bayesian tree places the Amed group as a distinct group within the Med clade A group too, with a posterior probability of 0.91 (Figure 2.10). The posterior probability value in this case means that the probability of a divergence between the Amed group and the Med clade A group is $91 \%$ certain, assuming that the model is correct, in all trees sampled. The two trees have very similar confidence that the two groups have diverged from each other, and that both groups have diverged from the other members of clade A (bootstrap value 58.1 and posterior probability 0.68 )

\section{$\underline{\text { 2.4 Discussion }}$}

All the anemones in this study, both from Europe and New Zealand, hosted Symbiodinium with ITS2 sequences very similar to, or identical to, either Symbiodinium sp. Amed or Symbiodinium sp. Mediterranean clade A. This also means that in this study all the Symbiodinium types within anemones from New Zealand and Europe were similar or identical to each other, suggesting that the Symbiodinium in A. aureoradiata are likely not endemic. Nearly all the A. aureoradiata from the latitudinal collection and all the anemones from the winter collection hosted Symbiodinium similar to, or identical, to Med clade A. However, an eighth of the summer collection, and nearly a quarter of the autumn collection, hosted Symbiodinium more similar to Amed. The preliminary comparison of the two habitats where seasonal collections were made, suggests that the presence of Amed, with seasonal change, occurs at both sites.

\subsubsection{Reliability of SSCP gels as a method of molecular identification}

Multiple SSCP bands in a sample could have been due to the conditions the gels were run in as mentioned in the results section, but also have been due to intragenomic variation (van Oppen et al. 2005). The number of base differences does not always correlate to distances between bands on a SSCP gel. These authors found that a small 
difference of bases in a sequence could finish up with a greater distance between bands on a gel compared to samples with a greater variation in bases, such as there would be between two clades. The eukaryotic nuclear rDNA region is repeated many times in a genome (Coleman 2003), and the ITS regions can be more variable than the $18 \mathrm{~S}, 28 \mathrm{~S}$ and 5.8S regions either side of them (Thornhill et al. 2007). The rapid rate of evolution in the ITS regions make them suitable for distinguishing between closely related taxa, but cannot always be relied upon (Coleman 2003) as the regions evolve relatively rapidly compared to the rest of the genome and this, combined with high levels of rDNA repetition, can lead to intragenomic and intraspecific variation (Stat et al. 2009). Remarkably, this variation can also be found within one Symbiodinium cell and can cause such 'messy' sequences after PCR amplification that they are unalignable, and can be so different that they could be identified as originating from different clades (van Oppen et al. 2005). Thornhill et al. (2007) found differing levels of intragenomic sequence variation when they cloned PCR products of amplified ITS2 regions from Symbiodinium types in clades A and E, the most ancestral of clades,. Some of these variants had base substitutions that resulted in different formations of the secondary structure, which would then travel and terminate at a different position on the SSCP gel. Thornhill et al. postulated that these were pseudogenes as secondary structure folding is an important part of the rDNA molecule and aides in the transcription into messenger RNA.

Multiple bands caused by intraspecific variation may come about by the sexual recombination of individual Symbiodinium cells within the same type. This theory was formulated by the discovery of variability in the genetic data of cultured isolates (Baillie et al. 2000, LaJeunesse 2001, Santos and Coffroth 2003). It was assumed that there would not be any variability in these cultures as they were made up of asexually reproduced cloned cells. However, Baillie et al. (2000) found more diversity within cultures of the northern hemisphere Symbiodinium A1 than has been seen in the sexually reproducing Gymnodinium catenatum. Sexual reproduction is probably more likely to occur in the free-living phase of Symbiodinium in the wild as meiosis has never been observed in hospite (LaJeunesse 2001, van Oppen and Gates 2006). Therefore, if sexual reproduction does occur in the Symbiodinium from A. aureoradiata, gametes should exist in the water column, as should free-living cells. Evidence for this has not 
been verified. A. aureoradiata is known to pass on symbionts to the next generation vertically from mother to larvae, but the larvae or adult could perhaps take up fresh symbionts from the environment. Sexual recombination would give rise to variation within a population, either within an individual host or within populations of cnidarian hosts in the environment (LaJeunesse 2001). This variation could most likely generate populations with the capacity to adapt to fluctuating temperature and irradiance, as is experienced in NZ. Evidence of this has been found by Howells et al. (2012), who discovered differences in sensitivity and tolerance to heat between populations belonging to the same Symbiodinium type, within the same host, the coral Acropora tenuis, when measured along a latitudinal gradient on the Great Barrier Reef.

More samples from NZ would need to be analysed to gauge whether there is a pattern of base substitution in the sequences from A. aureoradiata with different latitude, season and habitat, or whether the differences are just random substitutions. The possible intraspecific variation of the Symbiodinium from the sampled anemones, as seen by the differences in the sequences, may be driven by adaptation in populations to local conditions, genetic isolation from other Symbiodinium types and short asexual generation times of the Symbiodinium within the host cells (Howells et al. 2012). This ecological specialization to variable environmental conditions would not just include the external habitat with its various temperature and irradiance levels, but also could depend on the location of the symbiont within the host (Finney et al. 2010). The host tissue could provide different levels of light attenuation to the symbiont but would probably not provide differences in temperature within A. aureoradiata, as the anemone is so small.

\subsubsection{Change of symbiont with latitude or season}

Analysis of the sequences was more difficult in the NZ anemones than the ones from Europe due to the numerous band patterns on the SSCP gels and the chromatograms on Geneious being "messy", i.e. the peaks often had more than one base signature per position. The difficulty in reading the chromatogram could originate from an anemone hosting both Amed and Med clade A. Though both types are more similar each other than to other types of clade A, there are still 14 base pairs along the ITS2 sequence that do not match up. There were too few samples in this study to make a statistical 
statement or predict a trend as to the distribution and change in ratio of Symbiodinium type within the anemones. However, there could be a possibility that there is a change from Med clade A to Amed with the warmer, sunnier seasons and to the warmer areas of NZ. In the autumn, Symbiodinium similar to Amed was present in approximately $25 \%$ or the sampled anemones, both from the rocky shore and the mudflat. In summer Symbiodinium ITS2 regions were only sequenced in eight anemones in total but one of these was identified as being similar to Amed. Could this be a case of symbiont "shuffling" in the anemone or "switching" from the environment for the holobiont to acclimate to warmer or colder conditions?

Shuffling is the most likely mechanism for a change in symbiont type. Only two studies have found that cnidarian hosts (anemone and an octocoral) recovered from bleaching by switching to new types from the environment (Kinzie et al. 2001, Lewis and Coffroth 2004) and in both papers the authors acknowledge that the presence and recovery of residual symbionts was a possibility. Switching is also unlikely in temperate waters due to the scarcity of Symbiodinium cells in the water column, and is particularly unlikely in A. aureoradiata as it maternally transmits Symbiodinium to the next generation (Muller-Parker and Davy 2001). A host harbouring multiple Symbiodinium types is not as rare as once thought (Silverstein et al. 2012). A. aureoradiata could harbour a small population of Amed during the winter, which becomes the more dominant type in warmer conditions that are perhaps more favourable to its well-being.

\subsubsection{Similarities in Symbiodinium types between Europe and New Zealand}

In their study of Anemonia viridis, Bythell et al. (1997) investigated the genetic variation in symbionts between intertidal and subtidal anemones in southwest England. Using PCR-RFLP of the small subunit rRNA (SSU rRNA) gene they found that the Symbiodinium cells at both depths/shore heights were not genetically different enough to be classified as different types and identified them as being a type within clade A. Savage et al. (2002a) likewise investigated the diversity of high latitude Symbiodinium types in Bermuda, the Mediterranean and NE Atlantic. They used the same PCR-RFLP techniques as Bythell et al. (1997) on the SSU rDNA, LSU rDNA and ITS1 sequences, and found that every sample of sea anemone they analysed from the NE Atlantic and the Mediterranean contained an undescribed group within clade A (Savage et al. 2002a). 
They named the group "Temperate A" and postulated that it could be endemic to the region. Visram et al. (2006) subsequently characterised the molecular diversity of the Symbiodinium in ten species of scleractinian coral, sea anemone and scyphomedusan from the Mediterranean. They also used PCR-RFLP methods on SSU and LSU rRNA genes and found that only one of the species analysed, the sea anemone Bunodeopsis strumosa, harboured clade B whereas all the other species harboured Temperate A. They agreed with the previous study that the phylotype is endemic to the area. Forcioli et al. (2011) studied whether there was molecular diversity of Temperate A in a gorgonian, Eunicella singularis, at different depths and found that there was not.

It is probable that the group Temperate A includes ITS2 types Med clade A and Amed. This assumption is made because molecular identification of Symbiodinium cells from the same host species using either PCR-RFLP or ITS2 amplification from the studies just mentioned, as well as from Hunter et al. (2007), Meron et al. (2012) and now my study identified them all as either being Temperate A, when using PCR-RFLP, or ITS2 types Med clade A and Amed (Table 2.7). This is because analysing the ITS2 region provides better phylogenetic resolution at or below a species level, whereas the SSU and LSU rDNA sequences do not give such subtle phylogenetic differentiation (LaJeunesse 2001) (Figure 2.11). If the Symbiodinium types in A. aureoradiata in this study are the same as the ones in NE Atlantic and the Mediterranean, it would contradict Savage et al.'s (2002a) and Visram et al.'s (2006) hypothesis that Temperate A is endemic to the NE Atlantic and the Mediterranean but, rather, it has a distribution within both the southern and northern hemispheres. To confirm this, another molecular assessment of the same samples collected in my study could be carried out using the PCR-RFLP method on the SSU rDNA, LSU rDNA and ITS1 sequences. 
Table 2.7: Comparison of Symbiodinium identification from different molecular sequence analyses in host anemones that come from the NE Atlantic and the Mediterranean. Data are from both the current and published studies.

\begin{tabular}{|c|c|c|c|c|c|}
\hline \multirow[b]{2}{*}{ Authors } & \multirow[b]{2}{*}{ Host species } & \multirow[b]{2}{*}{ Molecule sequence analysed } & \multicolumn{3}{|c|}{ Molecular identification of Symbiodinium cells } \\
\hline & & & Temperate A & Med clade A & Amed \\
\hline Bythell et al. (1997) & \multirow{4}{*}{ Anemonia viridis } & SSU rRNA & $\checkmark$ & & \\
\hline Savage et al. (2002) & & SSU, LSU and ITS1 rDNA & $\checkmark$ & & \\
\hline Visram et al. (2006) & & LSU rRNA & $\checkmark$ & & \\
\hline This study & & ITS2 & & & $\checkmark$ \\
\hline Savage et al. (2002) & \multirow{3}{*}{ Cereus pedunculatus } & SSU, LSU and ITS1 rDNA & $\checkmark$ & & \\
\hline Visram et al. (2006) & & LSU rRNA & $\checkmark$ & & \\
\hline This study & & ITS2 & & & $\checkmark$ \\
\hline Visram et al. (2006) & \multirow[b]{2}{*}{ Balanophyllia europaea } & LSU rRNA & $\checkmark$ & & \\
\hline Meron et al. (2012) & & ITS2 & & $\checkmark$ & \\
\hline
\end{tabular}




\section{Level of diversity}

identified

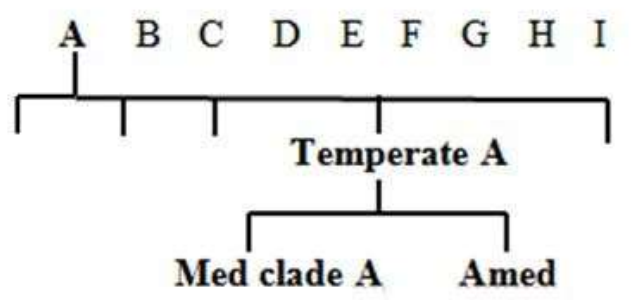

18S-rDNA

Clade

SSU rDNA, LSU rDNA and ITS1

Within clade

ITS2

Types

Figure 2.11: Diagrammatic representation of the different levels of phylogenetic resolution identified by the molecules analysed in Symbiodinium cells.

If Temperate A does indeed include both Med clade A and Amed, it demonstrates that these two similar types have a wide range of tolerance of similar climatic and light conditions found in northern and southern temperate regions. In the northern studies there was no significant genetic variation in the symbionts of $A$. viridis between 1-10 $\mathrm{m}$ depth (Bythell et al. 1997) or in the Mediterranean sea whip between 10-50 m (Focioli et al. 2011) or over a latitudinal range of approximately $15^{\circ}$. The Symbiodinium cells in the anemones in the current study were collected over a $12^{\circ}$ range and from quite varied habitats. Temperate Symbiodinium have been found in past studies to be less sensitive to variations in light and temperature than tropical types (Bythell et al. 1997). This may be because they are able to physiologically tolerate large variations in environmental parameters or possibly because the host is able to sustain them more in non-favourable conditions than can tropical hosts (Muller-Parker and Davy 2001).

\subsubsection{Symbiodinium diversity within temperate anemones}

There appears to be a low level of Symbiodinium diversity and high host specificity within temperate anemones. Those that are present appear to withstand the wide range of environmental conditions faced at different latitudes (Sanders and Palumbi 2011) and in different habitats (Bythell et al. 1997, Focioli et al. 2011). Being able to maintain a stable symbiotic partnership in highly-variable conditions may have been selected for, thereby providing evolutionary success to just a few Symbiodinium types (RodriguezLanetty et al. 2003) in high latitude anemones. This successful partnership is then maintained by the maternal transmission of symbionts to the next generation by the 
anemone (Muller-Parker and Davy 2001). This appears to be unlike the relationship between high-latitude corals and their symbionts. For example, a high diversity of Symbiodinium types and reduced host/symbiont specificity was found around the subtropical Lord Howe Island. On these reefs, changing symbiont types is thought to occur as an acclimatory mechanism to cope with changeable and stressful conditions (Wicks 2009).

Other temperate Symbiodinium have also been found to have a large biogeographical range. Two sea-anemone species, Anthopleura japonica and Anthopleura kurogane from Korea, harboured Symbiodinium in clade A for a latitudinal range of $4^{\circ}$ along the east coast of Korea to Cheju Island, and did so regardless of season (Rodriguez-Lanetty et al. 2000). This was also true for Symbiodinium muscatinei in A. elegantissima (Sanders and Palumbi 2011). Several authors postulate that some Symbiodinium types have a global distribution; for example with the same type being found in the Caribbean and the Red Sea (LaJeunesse 2001). Some hypothesise that global distribution may be related to temperature climes present at certain latitudes (LaJeunesse and Trench 2000, Gou et al. 2003). For example, Gou et al. (2003) identified a free-living dinoflagellate from China as a Symbiodinium sp. that was so similar in molecular identity to $S$. californium from California (USA) and a free-living strain (Gymnodinium varians) from Wellington (NZ) that they could possibly be all one species. If this is the case, and if the Symbiodinium in A. aureoradiata are the same as from the NE Atlantic and Mediterranean, the intriguing questions would be how these dinoflagellates are spread from the northern hemisphere to the southern (or vice versa), crossing tropical waters which would probably be too warm for them to tolerate. Do they have host species that act as vectors across the Equator? Or are the clades so ancient that they spread across their global distribution millions of years ago before mass divergence of the genus Symbiodinium into all the other clades? (This is discussed further in Chapter 4). To answer some of these questions more analysis needs to be carried out on much larger sample sizes, both in NZ and in Europe, between the different habitats, during the different seasons and across a range of latitudes. To complement the molecular study, a comparison of symbiont morphology and physiology could be made. And to differentiate if there is 'shuffling' of Amed or Med clade A within individual anemones, more sensitive molecular methods, such as cloning single Symbiodinium cells from a 
host sample, sequence analysis of microsatellite flanking regions or running real-time quantitative PCR, need to be undertaken. 


\section{Chapter 3}

\section{Photophysiology of isolated Symbiodinium cells from the anemone, Anthopleura aureoradiata}

\subsection{Introduction}

The success of the cnidarian-Symbiodinium symbiosis relies on the flux of nutrients between the host and symbiont. Organic matter produced by photosynthesis within the Symbiodinium cell supports the host's metabolism, growth and reproduction, and, in return, inorganic waste excreted by the host sustains the symbiont and the photosynthetic process (Muscatine and Kaplan 1994, Dubinsky and Berman-Frank 2001, Rodriguez-Lanetty et al. 2006). This partnership is especially successful in the tropics and has led to the dominance of reef-building corals, but it is also found across temperate and subtropical regions (Muller-Parker and Davy 2001, Savage et al. 2002a).

The genus Symbiodinium is highly diverse, consisting of nine clades (A-I) (Pochon and Gates 2010) with hundreds of ITS types identified (LaJeunesse 2001, Silverstein et al. 2012). The different ITS types are not equally susceptible to environmental stress (Iglesias-Prieto and Trench 1994, Tchernov et al. 2004, Hennige et al. 2008). Early hypotheses were that each clade may have a particular ecological characteristic, such as being tolerant or sensitive to certain thermal or photic conditions (Toller et al. 2001, LaJeunesse 2002, Savage et al. 2002c, Rowan 2004, Coffroth and Santos 2005). Instead, however, there seems to be vast photophysiological diversity within clades (Tchernov et al. 2004, Robinson and Warner 2006, Hennige et al. 2008, Suggett 2008, Fitt et al. 2009, Diaz-Almeyda et al. 2011, Buxton et al. 2012) and even within types (Howells et al. 2012). 


\subsubsection{Light and the photosynthetic apparatus}

Light provides the essential source of energy required for photosynthesis. However, the complex photosynthetic apparatus that converts light energy into chemical energy is vulnerable to damage when the quantity of light photons supplied is more than can be utilized for $\mathrm{CO}_{2}$ assimilation (Kraus and Weis 1991, Muller et al. 2001, Hill et al. 2005, Szabo et al. 2005, Warner and Berry-Lowe 2006). Photoinhibition and inactivation of photosystem II (PSII) comes about by several processes that may include degradation of the D1 protein at a rate that exceeds its rate of repair (Smith et al. 2005, Takahashi et al. 2009, Hill et al. 2011), damage to the repair system itself (Takahashi et al. 2004), inhibition of the synthesis of new intrinsic light-harvesting antennae (chlorophyll $a$ chlorophyll $c_{2}$-peridinin protein complexes (acpPC) and carotenoids) (Burton 1990, Takahashi et al. 2004, Venn et al. 2006), damage to the thylakoid membranes causing uncoupling of electron transport due to membrane-lipid composition changes (Tchernov et al. 2004), and impairment to the enzymes of the Calvin-Benson Cycle (Jones et al. 1998, Hoegh-Guldberg and Jones 1999, Jones 2004). The D1 protein is most susceptible to damage as it is the site of the electron transfer chain in PSII (Kern and Guskov 2011). Regardless of the processes involved, the excess energy in the photosystem leads to the production of reactive oxygen species (ROS) which may lead to further disruption and impairment of photophysiological processes if not detoxified (Venn et al. 2008a, Wicks et al. 2010b). High light alone can cause up to $40 \%$ reduction in the photosynthetic process (photoinhibition) in shallow reef corals (Gorbunov et al. 2001) but often light reacts synergistically with temperature, either high or low, to exacerbate photosystem damage (Jones et al. 1998, Saxby et al. 2003, Smith et al. 2005).

\subsubsection{Photoprotection}

All photosynthetic organisms need to balance their rate of $\mathrm{CO}_{2}$ assimilation in changing light conditions with the rate of damage and repair to the photosystems (Hill et al. 2011). Prevention from long term damage (chronic photoinhibition) to the photosystems can be accomplished through photoacclimation, a process of phenotypic modification of the light-harvesting apparatus in response to light (Hennige et al. 2008). Modifications can be through changes to the components of the light-harvesting antennae and to the size and number of the reaction-centre pools (Iglesias-Prieto and Trench 1994, Reynolds 
et al. 2008, Hennige et al. 2009). Recovery from chronic photoinhibition is slow and may take many days or longer (Gorbunov et al. 2001). Conversely, relatively rapid recovery from damage to, and then renewal of the D1 protein is termed "dynamic photoinhibition" and can take just minutes to a few hours (Gorbunov et al. 2001). Furthermore, short-term decline in photosynthetic efficiency during inclement conditions may occur not from damage, but by the photosystem "down-regulating" or protecting itself, by diverting photon energy away from the photosynthetic apparatus (Fitt et al. 2001b). Instead of the energy being used for photosynthetic reactions (photochemical quenching, qP), it is dissipated away from the photosystems as heat by non-photochemical quenching (NPQ) (Horton and Hague 1988). There are three major processes of NPQ: energy-dependent quenching (qE), quenching from state transitions by phosphorylation of light harvesting complexes (LHC) in PSII (qT), and photoinhibitory quenching (qI).

Quenching by qE is a feedback control mechanism generated when more light photons are absorbed than can be used by photosynthesis (Hill et al. 2005). Excessive photon absorption leads to increased protons in the thylakoid lumen which in turn build up the transthylakoid $\mathrm{pH}$ gradient $(\Delta \mathrm{pH})$. This acidification of the thylakoid lumen activates the conversion of diatoxanthin (Dtx) from the xanthophyll pigment diadinoxanthin (Ddx) (Goss et al. 2006, Schubert et al. 2006), which dissipates excess energy as heat (Brown et al. 1999, Hill et al. 2011). The $\Delta \mathrm{pH}$ can relax within seconds to minutes (Horton and Hague 1988) and can be so effective that it thermally dissipates energy 4-5 times faster than can be used for photosynthesis (Gorbunov et al. 2001).

State transitioning (qT) is where irradiance induces phosphorylation of the LHC in PSII, so that there is a rearrangement of the acpPC, as some components migrate to PSI. This redirects some of the excitation energy away from PSII, thereby protecting it (Gorbunov et al. 2001, Hill et al. 2005, Smith et al. 2005). This component of NPQ is able to relax over minutes once the irradiance has been removed (Horton and Hague 1988, Hill et al. 2005).

The third component of NPQ, photoinhibitory quenching (qI), relaxes very slowly once irradiance is removed, taking tens of minutes to hours, and remaining after $\Delta \mathrm{pH}$ has dissipated and qT has finished (Hill et al. 2005). This quenching leads to reversible 
photo-damage of the photosynthetic apparatus (Kraus and Weis 1991, Muller et al. 2001, Hill et al. 2005, Warner and Berry-Lowe 2006). Decreased photosynthetic activity at this point is mainly due to long-term down-regulation of PSII by damage to the D1 protein (Krause 1988, Muller et al. 2001).

Once there is damage to the photosystems from deleterious conditions, dysfunction to the symbiotic relationship sets in, which can lead to expulsion of the Symbiodinium cells from the host (Fitt et al. 2001a, Smith et al. 2005, Hill et al. 2009). This 'bleaching' phenomenon has become a major source of coral degradation and mortality in tropical seas, where changes of as little as 1-2 ${ }^{\circ} \mathrm{C}$ above or below average temperatures for an extended period, combined with high irradiance, can trigger the chain of cellular events that leads to symbiosis dysfunction (Hoegh-Guldberg 1999, Saxby et al. 2003).

\subsubsection{Temperate resilience}

In temperate regions where temperature, light intensity and duration of daylight are extremely variable in comparison to the tropics, the cnidarian-Symbiodinium relationship appears to be more resilient to environmental fluctuations. Some temperate anemones that host the same Symbiodinium type, function effectively across a wide latitudinal range and throughout the seasons (Bythell et al. 1997, Rodriguez-Lanetty et al. 2000, Sanders and Palumbi 2011). For example, the New Zealand anemone Anthopleura aureoradiata has not been seen to bleach in the wild (S. Davy. personal communication). This anemone is found throughout NZ, ranging from $34^{\circ} \mathrm{S}$ to $47^{\circ} \mathrm{S}$, on rocky shores and mudflats, where it is usually found attached to cockles. The mean seasonal air temperatures in $\mathrm{NZ}$ range from $19{ }^{\circ} \mathrm{C}$ in the summer to $6.5^{\circ} \mathrm{C}$ in the winter, though the lowest and the highest temperatures range from $-4{ }^{\circ} \mathrm{C}$ in the south to $34{ }^{\circ} \mathrm{C}$ in the north (see Appendix 1). Solar irradiance and UV radiation levels can also be extremely high, especially for intertidal organisms. Of particular note, A. aureoradiata harbours just two Symbiodinium types across its broad latitudinal range, with just one type being apparent in nearly $97 \%$ of anemones (Chapter 2). This interesting observation raises the question of how these symbionts withstand such a broad environmental range without compromising their photosynthetic function and hence the success of the symbiosis. Furthermore, this apparent resistance to bleaching provides a 
valuable parallel to more susceptible tropical corals and may provide important insight into which processes are significant in conferring environmental tolerance. This chapter addresses this question.

\subsubsection{Aim of the study}

This study aimed to establish whether the dinoflagellate symbiont, under high light at both low and high temperatures, has a strong capacity for photoprotection, and to test the hypothesis that the photosystem can down-regulate quickly and effectively to decrease the possibility of photodamage, resulting in a decrease in photosynthetic yield. This was tested by measuring chlorophyll $a$ fluorescence with a Maxi Imaging-Pulse Amplitude Modulated (I-PAM) (Walz, Effiltrich, Germany) fluorometer; this equipment allowed for non-destructive and rapid measurement of the same samples over time (Hill et al. 2005, Ralph et al. 2010). The photosynthetic rate of PSII, energy quenching by NPQ, and photosystem recovery were measured.

\subsection{Materials and Methods}

\subsubsection{Sampling}

Anemones (Anthopleura aureoradiata, $\mathrm{n}=120$ ) were collected on five occasions through the Austral summer of 2012 (end of January, and through February) from Pauatahanui Inlet, Wellington $\left(41^{\circ} 06^{\prime} 005^{\prime} ' \mathrm{~S}, 174^{\circ} 52^{\prime} 017^{\prime}\right.$ 'E). The temperature and solar radiation profiles for Pauatahanui were collated for the five years before collection to determine the temperature range in the anemone's habitat and thereby determine the conditions to use in the experiment (Figure 3.1).

The anemones were scraped off their host cockles and kept in plastic containers $(40 \mathrm{~cm}$ x $15 \mathrm{~cm} \times 15 \mathrm{~cm}$ ) in seawater. In the laboratory, the anemones were maintained in $1-\mu \mathrm{m}$ filtered seawater (FSW) in a temperature controlled room at $16^{\circ} \pm 1{ }^{\circ} \mathrm{C}$ on a 12 hour light/ 12 hour dark cycle (irradiance of $100 \pm 10 \mu \mathrm{mol}$ photons $\mathrm{m}^{-2} \mathrm{~s}^{-1}$ ) for $2-3$ weeks to acclimatize to the control experimental conditions. The anemones were fed five times per week with Artemia sp. nauplii and the water changed soon after feeding. 

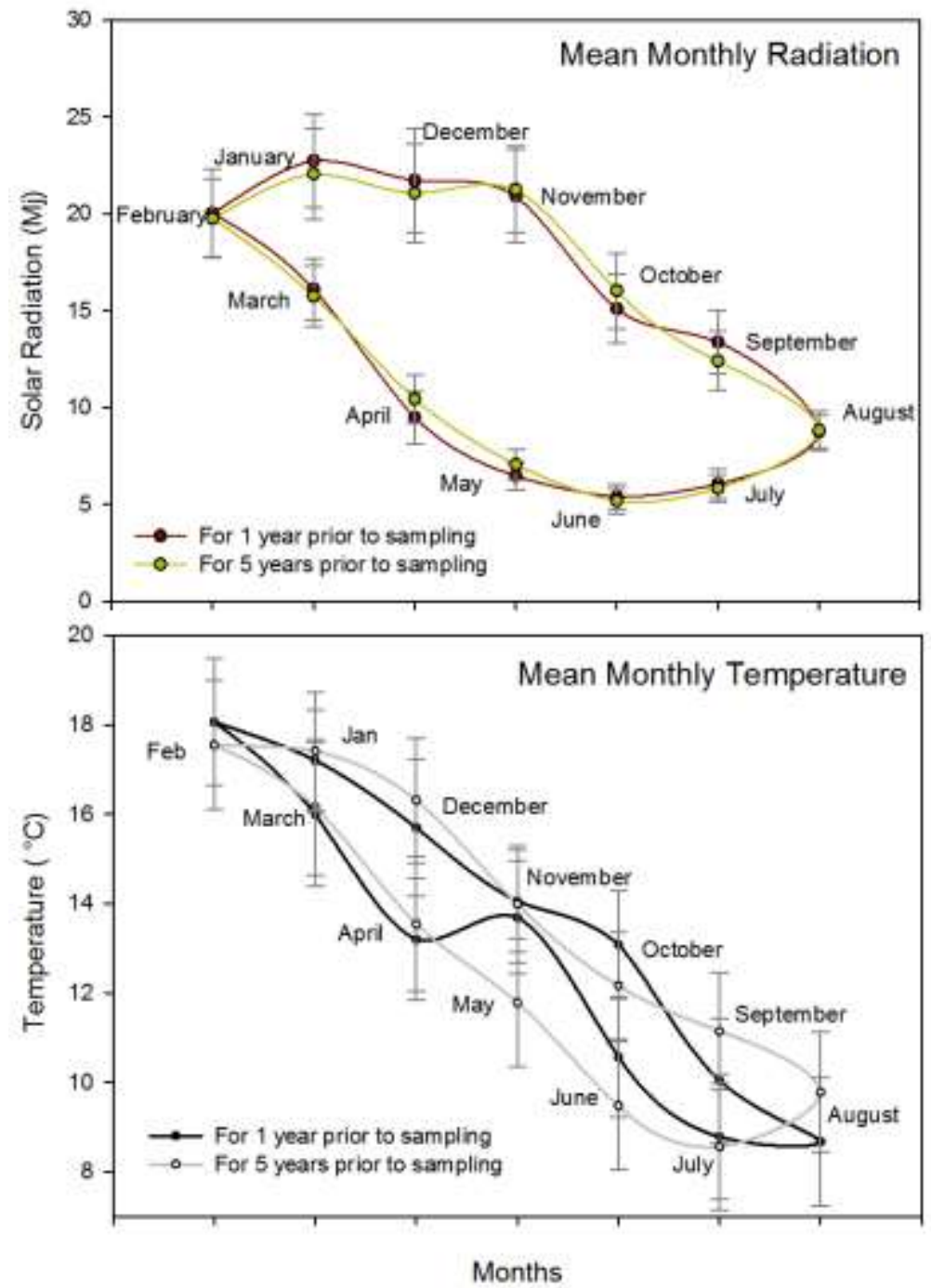

Figure 3.1: Monthly temperature and solar irradiance (mean \pm SE) near Pauatahanui Inlet. Data were collected from the National Climate Database run by the National Institute of Water and Atmospheric Research Ltd (NIWA). Data came from a weather station in Paraparaumu (25 km from Pauatahanui). The daily highest and lowest temperatures, and the global diffuse and direct solar radiation data for one year prior and five years prior to sampling were used.

\subsubsection{Experimental set-up}

Three tanks were maintained at either $30 \pm 0.1{ }^{\circ} \mathrm{C}$ (warm), $16 \pm 1{ }^{\circ} \mathrm{C}$ (control) or $8 \pm$ $0.2{ }^{\circ} \mathrm{C}$ (cold). Water was heated and maintained in the warm tank by a HAAKE C10 water heater, and the control and cold tanks were cooled and maintained by a HAAKE EK20 immersion cooler. There were two light levels per tank: high light (400 $\pm 20 \mu \mathrm{mol}$ photons $\left.\mathrm{m}^{-2} \mathrm{~s}^{-1}\right)$ and low light $\left(100 \pm 20 \mu \mathrm{mol}\right.$ photons $\left.\mathrm{m}^{-2} \mathrm{~s}^{-1}\right)$. These irradiances were 
those incident on the surface of the experimental tubes, as measured using a LI-COR LI-250A light meter. The experiment therefore had six treatments with two factors, light (high and low) and temperature (8 (cold), 16 (moderate) and $30{ }^{\circ} \mathrm{C}$ (warm)). The treatments will be referred to in figures as: Low light, $8^{\circ} \mathrm{C}(\mathrm{L} 8)$; low light, $16^{\circ} \mathrm{C}(\mathrm{L} 16)$; low light, $30{ }^{\circ} \mathrm{C}(\mathrm{L} 30)$; high light, $8{ }^{\circ} \mathrm{C}(\mathrm{H} 8)$; high light, $16^{\circ} \mathrm{C}(\mathrm{H} 16)$; and high light, $30{ }^{\circ} \mathrm{C}(\mathrm{H} 30)$.

\subsubsection{Experimental procedure}

The original intention of the study was to measure the photophysiology of the symbionts within the host as a true measure of how the holobiont responds to stress. This proved too difficult due to the frequent and unpredictable movement of the anemones (see Appendix 3). Therefore, to study the photophysiology of the symbionts, the Symbiodinium cells were isolated from the host.

A total of 105 anemones were blended simultaneously with FSW in a Breville BFP50 Whizz $^{\mathrm{TM}}$ Chopper and Shake blender for 30 seconds. This tissue slurry was then homogenised further with a hand-held glass tissue grinder. The homogenate was made up to $200 \mathrm{ml}$ with FSW and centrifuged (500 x g, 5 minutes), before the supernatant was decanted, and the algal pellet re-suspended in FSW (Figure 3.2); this procedure was repeated a further five times to wash the algal cells of host cell debris.
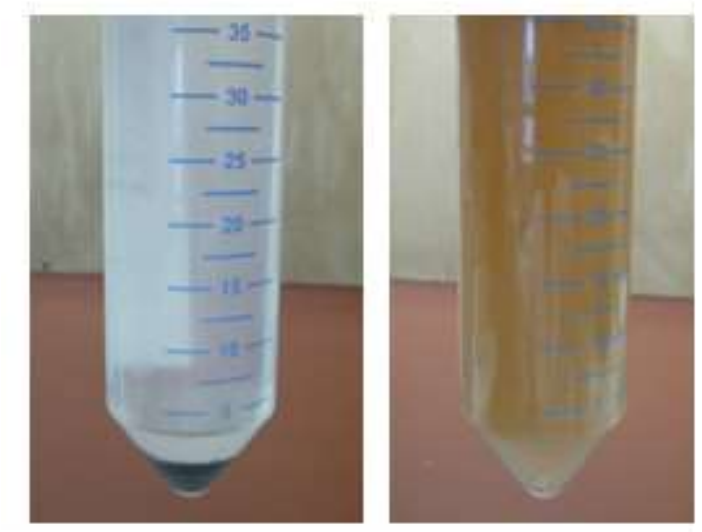

Figure 3.2: Algal pellet after centrifuging and algal suspension once re-suspended in FSW 
The algae were then re-suspended in $128 \mathrm{ml}$ of FSW and decanted into a $150 \mathrm{ml}$ flask. The flask was returned to the temperature-controlled room under the low light regime for 3 hours. Preliminary studies indicated that the algal photosynthetic efficiency of PSII dropped for the first two hours after isolation before recovering, and that the cells remained viable for at least 12 hours after isolation (Appendix 4).

After this time, $0.5 \mathrm{ml}$ of algal suspension was removed for cell counts, to measure the cell density at the onset of the experiment (see later). A sub-sample $(20 \mathrm{ml})$ of the suspension was also pipetted into six 50-ml Falcon tubes. Three Falcon tubes containing the algae destined for the low-light treatments (i.e. L8, L16, L30) were attached to wire brackets by elastic bands, with one Falcon tube placed into each of the three tanks. The three Falcon tubes containing algae destined for the high-light treatments (i.e. H8, H16, H30) were placed into position one hour later. This difference in timing was necessary as I-PAM fluorometer measurements for each light treatment, including preparation time, took $50 \mathrm{~min}$. The flask with the remaining suspension was dark adapted for 20 min. A 1-ml aliquot of algal suspension was then pipetted from the flask into each of 5 x 3-ml darkened cylindrical vials. These vials were then placed into a black, wooden rack and placed into position on the I-PAM stage for measurement.

An hour and 40 min after being placed in the tanks, the Falcon tubes were wrapped in aluminium-foil and returned to their respective tanks to dark adapt for 20 min, thereby maintaining their treatment temperatures. Aliquots of algal suspension were then pipetted into vials as described above. Once all 15 vials per light treatment (i.e. L8, L16 and L30, or H8, H16 and H30) were in place, the rack was positioned on the I-PAM stage (Figure 3.3). This protocol was carried out two more times for each treatment, so that I-PAM measurements were made 2, 4 and $6 \mathrm{~h}$ after the Symbiodinium cell suspension was placed in its respective treatment tank. At the end or the replicate run, $0.5 \mathrm{ml}$ from each Falcon tube was collected and immediately frozen at $-80^{\circ} \mathrm{C}$ for future cell counts. The mean of the five I-PAM data measurements for each replicate cell suspension/treatment were averaged for each time point. Each treatment was repeated four times over a period of four weeks. 


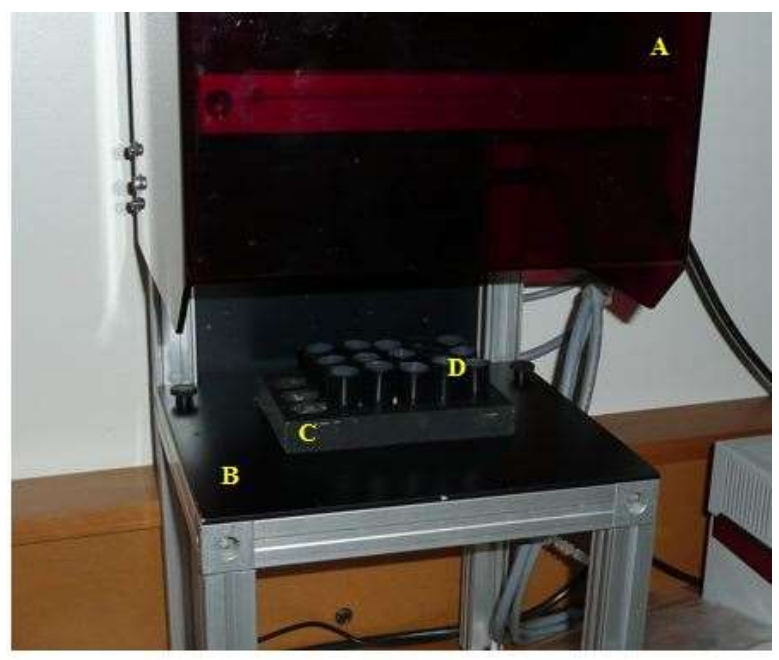

Figure 3.3: Set-up of the I-PAM and the 15 vials measured for each light treatment at each time point. A) Raised red-perspex hood of illumination unit; B) stage on the mounting stand; C) Black rack; D) Darkened 3-ml vials.

\subsubsection{Cell counts}

Symbiont densities at the beginning and end of the experiment were estimated by using a haemocytometer (Improved Neubauer). Each sample was counted 24 times and averaged.

\subsubsection{Photophysiological measurements}

Measurements of chlorophyll fluorescence were carried out with a Maxi I-PAM (Figure 3.4) (settings: measuring intensity $=3$, frequency $=1$, gain $=4$, damping $=3 \mathrm{NIR}=8$, saturation pulse intensity $=10$ ).

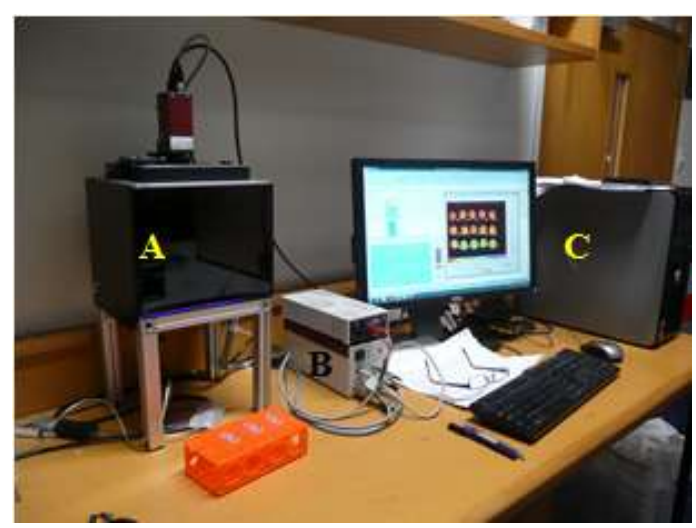

Figure 3.4: Imaging-PAM set-up: A) LED-array illumination unit; B) control unit; C) computer with ImagingWin-software 
The first I-PAM measurement after dark adaptation was a fast dark-light induction curve (settings: delay before actinic light activated $=10$ seconds, clock-time between saturation pulses $=60$ seconds, duration of curve $=615$ seconds, actinic light setting $=6$ for low light $\left(111 \mu \mathrm{mol}\right.$ photons $\left.\mathrm{m}^{-2} \mathrm{~s}^{-1}\right)$ and 12 for high light $\left(396 \mu \mathrm{mol}\right.$ photons $\mathrm{m}^{-2} \mathrm{~s}^{-}$ $\left.{ }^{1}\right)$ ). The irradiance of actinic light depended on whether the samples came from the lowlight treatments or the high-light treatments. Information collected gave the minimal fluorescence yield before the first saturation pulse $\left(\mathrm{F}_{\mathrm{o}}\right)$, the dark-adapted maximum fluorescence yield at the peak of the first pulse $\left(\mathrm{F}_{\mathrm{m}}\right)$, the instantaneous fluorescence yield throughout the induction curve $\left(\mathrm{F}_{\mathrm{t}}\right)$, and the light-adapted maximum fluorescence yield at the peak of all subsequent pulses $\left(\mathrm{F}_{\mathrm{m}}{ }^{\prime}\right)$.

Using the data collected, the following parameters were calculated according to the equations of Genty et al. (1989) and Schreiber et al. (1994):

Photosynthetic efficiency $=$ maximum quantum yield $=\left(\mathrm{F}_{\mathrm{m}}-\mathrm{F}_{\mathrm{o}}\right) / \mathrm{F}_{\mathrm{m}}=\mathrm{F}_{\mathrm{v}} / \mathrm{F}_{\mathrm{m}}[$ Eq. 1]

Effective PSII quantum yield $\left(\phi_{\mathrm{PS}}\right)=\left(\mathrm{F}_{\mathrm{m}}{ }^{\prime}-\mathrm{F}\right) / \mathrm{F}_{\mathrm{m}}{ }^{\prime}=\Delta \mathrm{F} / \mathrm{F}_{\mathrm{m}}{ }^{\prime}[$ [Eq. 2]

Coefficient of NPQ (qN) $=\left(\mathrm{F}_{\mathrm{m}}-\mathrm{F}_{\mathrm{m}}{ }^{\prime}\right) /\left(\mathrm{F}_{\mathrm{m}}-\mathrm{F}_{\mathrm{o}}\right)[\mathrm{Eq} .3]$

Immediately after the dark-light induction curve, a rapid-light curve (RLC) was recorded. A RLC is best run without samples being dark-adapted as it should not be dominated by induction effects (Heinz Walz GmbH 2006); the best time to run a RLC is after a dark-light induction curve, so retaining the original $\mathrm{F}_{\mathrm{v}} / \mathrm{F}_{\mathrm{m}}$. Measurements of $\mathrm{F}$ and $F_{m}$ ' were obtained through the application of a series of saturation pulses under increasing and then decreasing actinic irradiance $(0,1,21,56,111,186,281,336,396$, 461, 531, 701 $\mu \mathrm{mol}$ photons $\mathrm{m}^{-2} \mathrm{~s}^{-1}$ and back down again). Each step of actinic irradiance was maintained for 60 seconds. Measurements during the decreasing irradiance steps indicated levels of recovery from exposure. From the data recorded, the following parameters were calculated:

(1) The electron transport rate (ETR). In terrestrial plants, this has been determined as $\phi_{\text {PSII }} \times 0.84 \times 0.5 \times$ PAR, where $\phi_{\text {PSII }}=\left(\mathrm{F}_{\mathrm{m}}{ }^{\prime}-\mathrm{F}\right) / \mathrm{F}_{\mathrm{m}}{ }^{\prime}$ and 0.84 and 0.5 are 
absorption coefficients. As these absorption coefficients are unknown for the isolated Symbiodinium cells, a relative ETR (rETR) was calculated from each saturation pulse, where $\mathrm{rETR}=\left(\mathrm{F}_{\mathrm{m}}{ }^{\prime}-\mathrm{F}\right) / \mathrm{F}_{\mathrm{m}}{ }^{\prime}[\mathrm{Eq} 4]($ Ralph et al. 2002) .

(2) Non-photochemical quenching (NPQ), where NPQ $=\left(F_{m}-F_{m}{ }^{\prime}\right) / F_{m}{ }^{\prime}[$ [Eq. 5]. $\mathrm{NPQ}_{\max }$ was determined as the highest NPQ value on the RLC (Hill et al. 2004).

To compare the descriptive parameters $\alpha$ (a measure of the efficiency of light utilization, i.e. light limited photosynthetic rate), $E_{k}$ (saturation radiance, i.e. the irradiance at the onset of saturation) and $\mathrm{rETR}_{\max }$ (the maximum observed relative electron transport rate), the RLCs were fitted by the function of Platt et al. (1980) using Sigmaplot 11.0 (Platt et al. 1980, Ralph et al. 2002, Hill et al. 2004) such that:

$P=P_{s}\left[1-\exp \left(-\alpha E_{d} / P_{s}\right)\right] \exp \left(-\beta E_{d} / P_{s}\right)[$ Eq. 6]

where $\mathrm{E}_{\mathrm{d}}$ is the downwelling irradiance, $P_{s}$ is a scaling parameter defined as the maximum potential ETR if there is no photoinhibition, $\alpha$ is the initial slope of the light curve before the onset of saturation, and $\beta$ is the slope of the light curve after the onset of photoinhibition or down-regulation (Figure 3.5). If there is no photoinhibition (i.e. $\beta=0$ ), then Equation 6 is simplified to:

$P=P_{m}\left[1-\exp \left(-\alpha E_{d} / P_{m}\right)\right][$ Eq. 7]

The parameters $\mathrm{rETR}_{\max }$ and $\mathrm{E}_{\mathrm{k}}$ were estimated using the equations:

$\mathrm{rETR}_{\max }=\operatorname{Ps}[\alpha /(\alpha+\beta)] /[\beta /(\alpha+\beta)]^{\beta / \alpha}[\mathrm{Eq} .8]$

$\mathrm{E}_{\mathrm{k}}=\mathrm{rETR}_{\max } / \alpha$ [Eq. 9] 


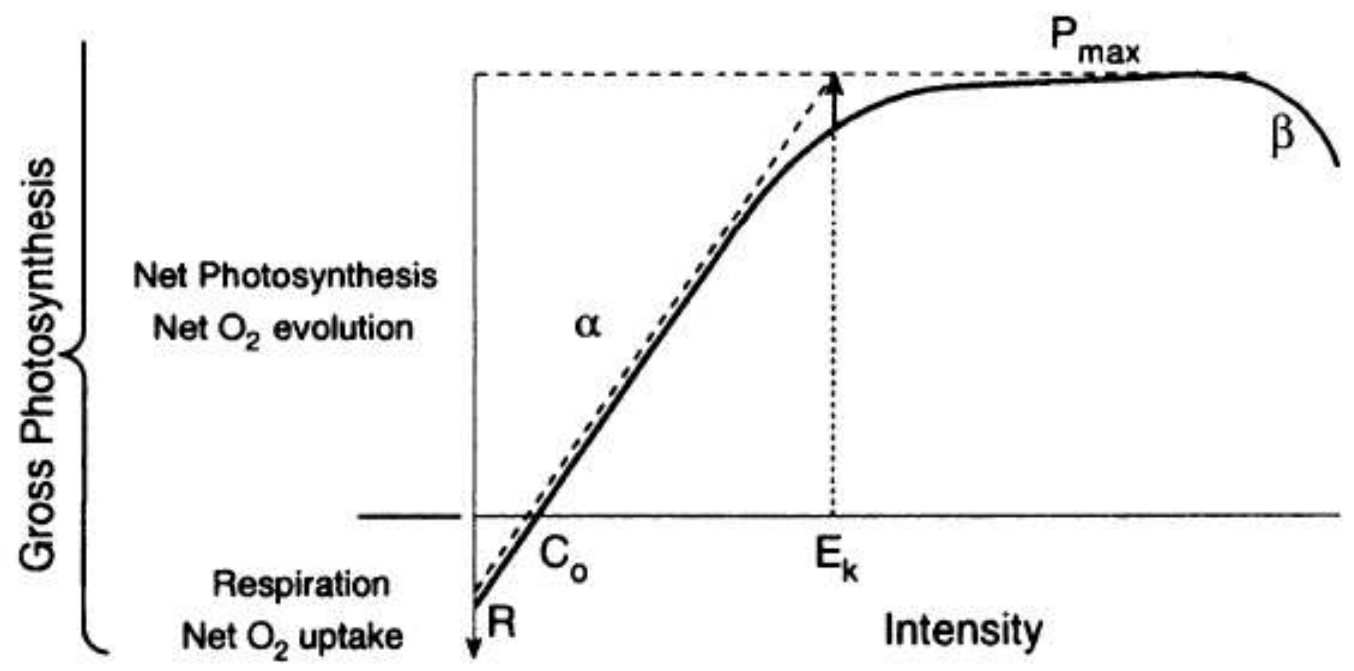

Figure 3.5: A typical photosynthesis versus irradiance (P-I) curve. In the dark, there is net consumption of oxygen $\left(\mathrm{C}_{\mathrm{o}}\right)$ as a result of respiration $(\mathrm{R})$. Photosynthetic rate increases linearly $(\alpha)$ with irradiance until the saturation intensity $\left(\mathrm{E}_{\mathrm{k}}\right)$ is reached and the rate plateaus at its maximum $\left(\mathrm{P}_{\max }\right)$. Past this optimum irradiance, a decline in photosynthetic rate often occurs $(\beta)$. Figure taken from Falkowski and Raven (2007).

\subsubsection{Statistical analysis}

For the final analysis, there were four replicates per treatment. However, if an outlier was found which could not be removed by transformation, this value was replaced with the mean of the other three replicates, as recommended by Quinn and Keough (2002). This occurred with about $5.5 \%$ of the data. Though not ideal, outliers could not just be removed because a repeated measure analysis cannot be performed if any values are missing.

Statistical analysis was carried out using PASW (version 18.00). Variation of fluorescence measurements was tested using a repeated measures one-way analysis of variance model (rmANOVA, $\alpha=0.05$ ) with time of measurement as the within-subject factor, and light and temperature as the between-subject factors. Where assumptions of normality and equal variance were not satisfied, transformation of data was carried out using an arcsin or a reflect and square root method. The Mauchley Sphericity Test was used in all analyses to test for sphericity of data. As all data met assumptions of sphericity, post hoc tests were carried out using Tukey HSD $(\alpha=0.05)$. 


\section{$\underline{\text { 3.3 Results }}$}

\subsubsection{Algal cell density}

There was a decrease of $22-37 \%$ in cell density from the start to the end of the experiment in all treatments, except in the high-light, warm treatment (Tukey post hoc, two-way ANOVA, $0.003 \leq \mathrm{p} \leq 0.044)$. At the end of the experiment there were no differences between the cell densities in any of the treatments (Tukey post hoc, two-way ANOVA, $0.625 \leq \mathrm{p} \leq 1.000$ : Figure 3.6) (see Appendix 5 for statistical data).

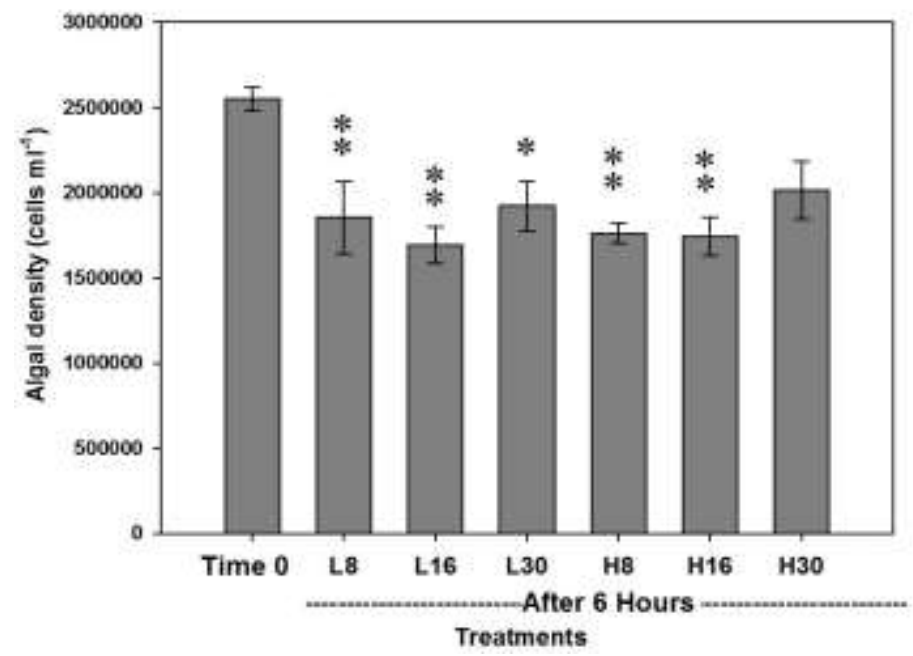

Figure 3.6: Density of cells at the beginning and the end of the experiment $(0-6 \mathrm{~h})($ mean $\pm \mathrm{SE}, \mathrm{n}=4)$. Notations mark treatments that are significantly different from the start measurements, where $* \mathrm{p}<0.05$ and $* \mathrm{p}<0.01$

\subsubsection{Fluorescence parameter measurements}

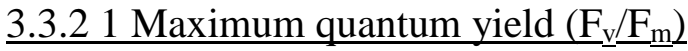

Changes in maximum quantum yield $\left(\mathrm{F}_{\mathrm{v}} / \mathrm{F}_{\mathrm{m}}\right)$ were evident after $2 \mathrm{~h}$, with cold temperature at both light levels having the greatest affect (rmANOVA, $\mathrm{p}<0.001$ ); $\mathrm{F}_{\mathrm{v}} / \mathrm{F}_{\mathrm{m}}$ decreased by $62 \%$ and $73 \%$ in the cold under low and high light (rmANOVA, $p<0.001$ in both treatments: Figure 3.7) (see Appendix 6 for all statistical data for this section). $\mathrm{F}_{\mathrm{v}} / \mathrm{F}_{\mathrm{m}}$ continued to decline in response to cold treatment between $2-4 \mathrm{~h}$, before stabilising in the low-light treatment and declining further in the high-light treatment between 4-6 h (Tukey post hoc, rmANOVA, $\mathrm{p}=0.001$ ). High light also had a negative impact in the first $2 \mathrm{~h}$, causing a decrease in $\mathrm{F}_{\mathrm{v}} / \mathrm{F}_{\mathrm{m}}$ in the other two temperature treatments (Tukey post hoc, rmANOVA, $\mathrm{p}<0.001$ for H16 and $\mathrm{p}<0.05$ for H30). $\mathrm{F}_{\mathrm{v}} / \mathrm{F}_{\mathrm{m}}$ in the control, low-light, moderate treatment (L16), and the low-light, warm 
treatment did not change over the $6 \mathrm{~h}$. At the end of the 6-h experiment, there was a difference between the control and the two cold treatments (Tukey post hoc, two-way ANOVA, $\mathrm{p}<0.001$ ), and the high-light, moderate treatment (Tukey post hoc, two-way ANOVA, $\mathrm{p}=0.009$ ).

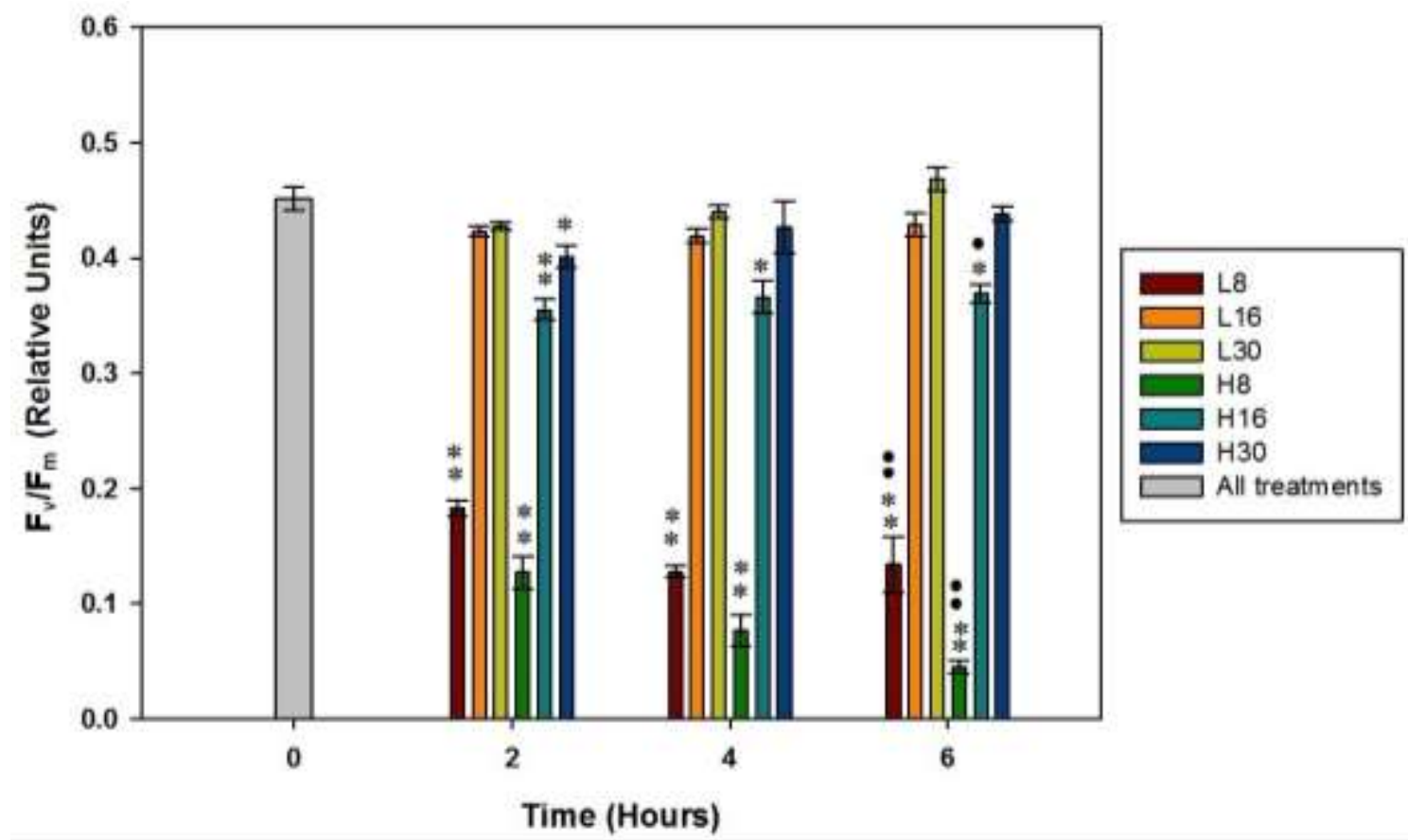

Figure 3.7: Maximum quantum yield $\left(\mathrm{F}_{\mathrm{v}} / \mathrm{F}_{\mathrm{m}}\right)$ from the start of a dark-light induction curve following 20 min of dark adaption (mean $\pm \mathrm{SE}, \mathrm{n}=4$ ). Measurements were taken at the start of the experiment and 2, 4 and $6 \mathrm{~h}$ after. Initial measurements came from the same algal suspension before the suspension was divided between the six treatments with two factors (temperature and light). Notations indicate $F_{v} / F_{m}$ that were different to the initial measurement (Tukey post hoc, rmANOVA, * p $<0.05$ and $*$ p $<0.001$ ), or difference in $\mathrm{F}_{\mathrm{v}} / \mathrm{F}_{\mathrm{m}}$ between treatments and the control (L16) at 6 hours (Tukey post hoc, two-way ANOVA, $\bullet \mathrm{p}<0.05$ and $\bullet \mathrm{p}<0.001)$.

\section{$\underline{\left.\text { 3.3.2.1 Effective PSII quantum yield ( } \Delta \mathrm{F} / \mathrm{Fm}{ }^{\prime}\right)}$}

The series of dark-light induction curves (Figure 3.8) clearly demonstrates more reduction, by the end of the experiment, of effective PSII quantum yield $\left(\Delta \mathrm{F} / \mathrm{F}_{\mathrm{m}}{ }^{\prime}\right)$ in response to high light at all temperatures compared with low light at the same temperature (Tukey post hoc, two-way ANOVA, $\mathrm{p} \leq 0.005$ ). $\Delta \mathrm{F} / \mathrm{F}_{\mathrm{m}}$ ' was present at all time-points in all of the low-light treatments. It decreased in the first $2 \mathrm{~h}$ in the control treatment (L16) (rmANOVA, $\mathrm{p}=0.009)$ but recovered and remained the same as the start measurement by 4 and $6 \mathrm{~h}$ (Tukey post hoc, two-way ANOVA, $0.059 \leq \mathrm{p} \leq 0.562$ ). 
In comparison, $\Delta \mathrm{F} / \mathrm{F}_{\mathrm{m}}$ ' was consistently higher in the low-light, warm treatment than in the control (Tukey post hoc, two-way ANOVA, p < 0.001) and increased over time (rmANOVA, $\mathrm{p}<0.001) . \Delta \mathrm{F} / \mathrm{F}_{\mathrm{m}}$ ' in the low-light, cold treatment declined below its initial value by $2 \mathrm{~h}$, but then remained constant (Tukey post hoc, two-way ANOVA, $\mathrm{p} \geq 0.952) \Delta \mathrm{F} / \mathrm{F}_{\mathrm{m}}$ ' was adversely affected by high light at all three temperatures (rmANOVA, $\mathrm{p}<0.05$ ). After $2 \mathrm{~h}$ of experimental treatment, and only after one minute of actinic light in the dark-induction curve, $\Delta \mathrm{F} / \mathrm{F}_{\mathrm{m}}$ ' had dropped to zero under high light at 16 and $30{ }^{\circ} \mathrm{C}$, though in the cold, high-light treatment this parameter remained just above zero. After $4 \mathrm{~h}$ under high light exposure, $\Delta \mathrm{F} / \mathrm{F}_{\mathrm{m}}$ ' was zero at all temperatures. A small recovery was measured at $6 \mathrm{~h}$, with $\Delta \mathrm{F} / \mathrm{F}_{\mathrm{m}}$ ' rising just above zero in the high-light. warm treatment.

\subsubsection{Coefficient of non-photochemical quenching ( $q N)$}

$\mathrm{qN}$ in all treatments, except the low-light, warm treatment, increased rapidly and then stabilized one minute after exposure to actinic light, at all time-points (Figure 3.8). qN decreased markedly by $2 \mathrm{~h}$ in the low-light, warm treatment (Tukey post hoc, two-way ANOVA, $\mathrm{p}=0.016$ ), and was less than $\mathrm{qN}$ in the control treatment (L16) (Tukey post hoc, two-way ANOVA, p < 0.001). Over the next $4 \mathrm{~h}$, in the low-light, warm treatment, $\mathrm{qN}$ continued to drop, mirroring the increase in $\Delta \mathrm{F} / \mathrm{F}_{\mathrm{m}}{ }^{\prime}(\mathrm{rmANOVA}, \mathrm{p}<0.001)$, and was less than in all other treatments by the end of the experiment (Tukey post hoc, twoway ANOVA, $\mathrm{p}<0.001$,). $\mathrm{qN}$ in the last measurement of the dark-induction curve, under low light in the cold and moderate treatments, was less than under high light at the corresponding temperatures at all time-points (Tukey post hoc, two-way ANOVA, $\mathrm{p}<0.001)$.

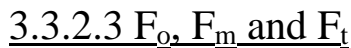

The series of graphs for the dark induction curves (Figure 3.8) show that the difference between $F_{o}$ and $F_{m}$ fluorescence remained relatively constant throughout the experiment for all of the moderate and warm treatments (mean difference between $F_{o}$ and $F_{m}$, 0.1951). In the two cold treatments, particularly under high light, both $F_{o}$ and $F_{m}$ dropped over time, as did the difference between the two (mean difference between $\mathrm{F}_{\mathrm{o}}$ and $\left.\mathrm{F}_{\mathrm{m}}, 0.0147\right)$. The values in the $\mathrm{F}_{\mathrm{t}}$ curves, which monitor the response of chlorophyll. 


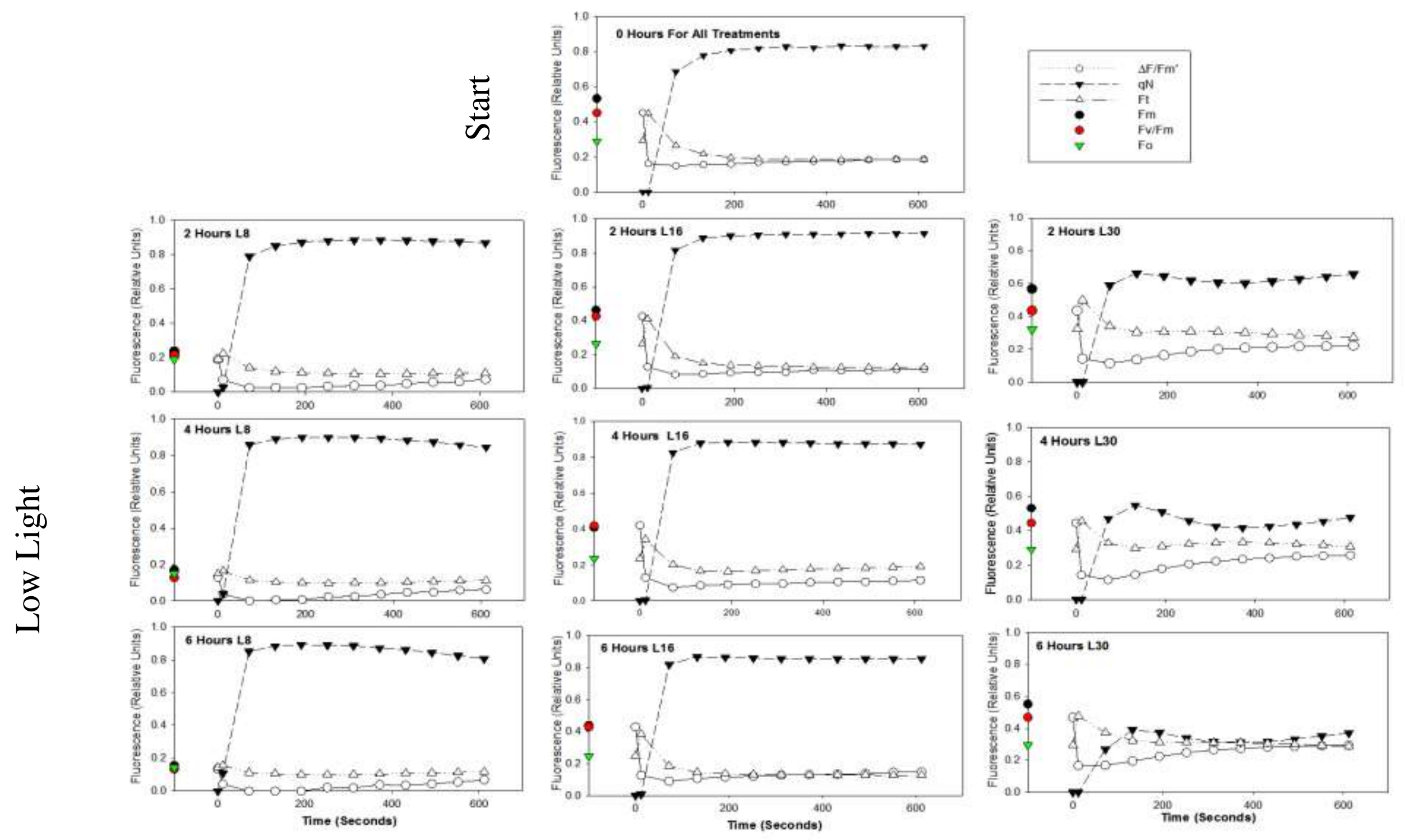



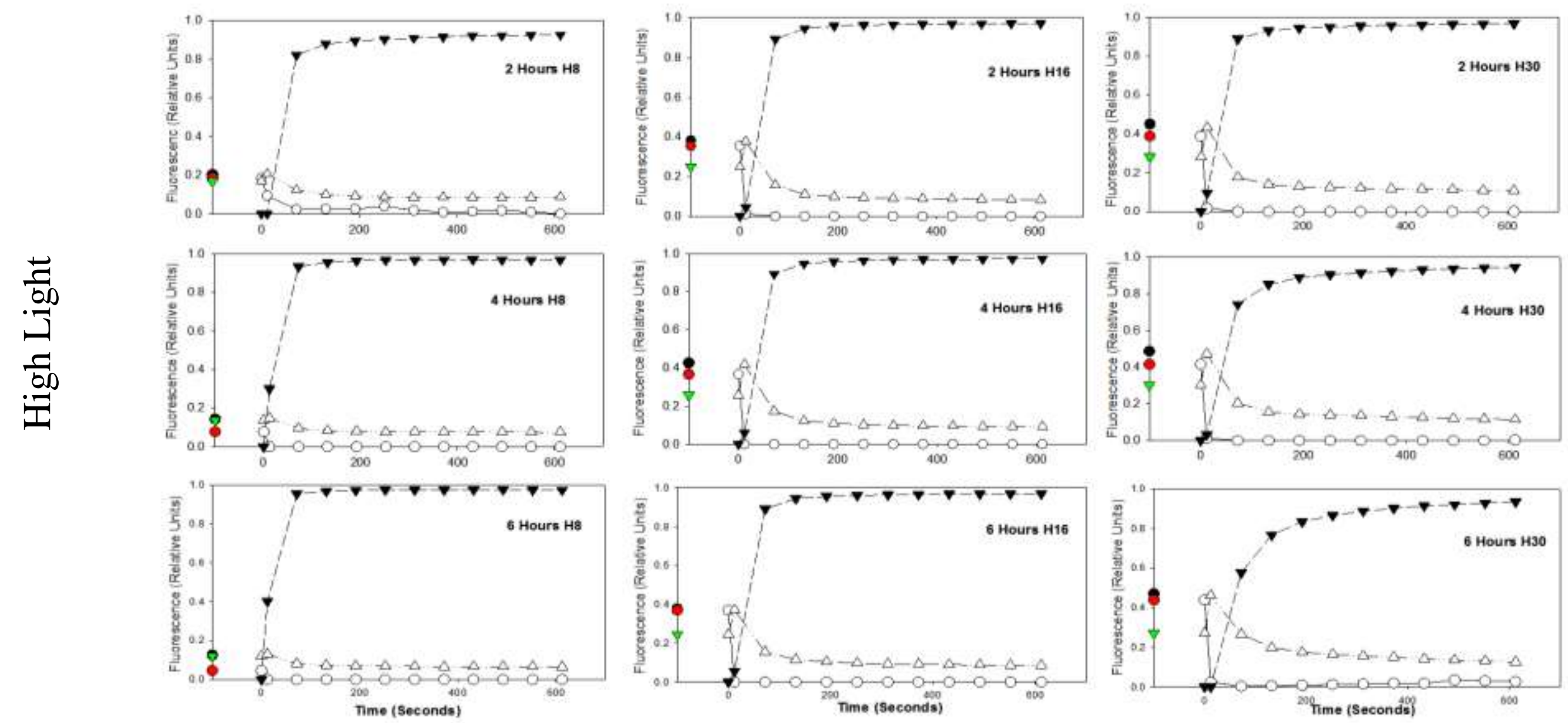

Figure 3.8: Fluorescence parameters through dark-induction measurements with the I-PAM taken at the start and after 2,4 and $6 \mathrm{~h}$ (mean, $\mathrm{n}=4$. For clarity, $\mathrm{SE}$ lines have been omitted but SE values can be found in Table A6.4.1 in Appendix 6).Treatments in the low light (first three rows) were measured separately from the treatments in high light (last three rows). Parameters measured were $\mathrm{F}_{\mathrm{m}}$ (dark-adapted maximum fluorescence yield), $\mathrm{F}_{\mathrm{o}}$ (minimum fluorescence yield) and $\mathrm{F}_{\mathrm{v}} / \mathrm{F}_{\mathrm{m}}$ (maximum quantum yield) at the start of the induction curve, followed by $\Delta \mathrm{F} / \mathrm{F}_{\mathrm{m}}{ }^{\prime}$ (effective PSII quantum yield), $\mathrm{qN}$ (non-photochemical quenching) and $\mathrm{F}_{\mathrm{t}}$ (instantaneous yield) throughout the induction curve with saturation pulses every minute for 10 minutes. Saturation pulses for low light were set at $111 \mu \mathrm{mol}$ photons $\mathrm{m}^{-2} \mathrm{~s}^{-1}$, and for high light, $396 \mu \mathrm{mol}$ photons $\mathrm{m}^{-2} \mathrm{~s}^{-1}$. 
fluorescence, were generally higher in the warmer treatments, and higher under low light.

\subsubsection{Maximum observed relative electron transport rate $\left(\mathrm{rETR}_{\max }\right)$}

$\mathrm{rETR}_{\max }$ was negatively affected by temperature and irradiance over the 6-h treatment (rmANOVA, $\mathrm{p}=0.01$ ). By $2 \mathrm{~h}, \mathrm{rETR}_{\max }$ in all treatments had decreased, except in the low-light, warm treatment, and more so under low temperature and high light (Tukey post hoc, rmANOVA, $0.001 \geq \mathrm{p} \leq 0.032$ ) (Figure 3.9). $\mathrm{rETR}_{\max }$ then increased between 2-6 $\mathrm{h}$ in both warm treatments, but more so in low light than high light (Tukey post hoc, rmANOVA, $\mathrm{p}<0.001$ in L30 and $\mathrm{p}=0.001$ in H30). This parameter remained relatively unchanged between $2-6 \mathrm{~h}$ in the other four treatments. At the end of the experiment, $\mathrm{rETR}_{\max }$ was greater than the control (L16) in both warm treatments (Tukey post hoc, two-way ANOVA, p $<0.001$ ), and less than the control in both cold treatments and the high-light, moderate treatment (Tukey post hoc, two-way ANOVA, $\mathrm{p}<0.001)$.

\subsubsection{Saturation irradiance $\left(\mathrm{E}_{\mathrm{k}}\right)$}

Changes to the saturation irradiance $\left(E_{k}\right)$ were affected by low light in the first $2 \mathrm{~h}$ (rmANOVA, $\mathrm{p}=0.002$ ) (Figure 3.9) and by temperature in the first $4 \mathrm{~h}$ of the experiment (rmANOVA, $\mathrm{p}<0.05$ ), with a decrease in $\mathrm{E}_{\mathrm{k}}$ in the cold treatment and an increase in the warm treatments. In the first $2 \mathrm{~h}$, only $\mathrm{E}_{\mathrm{k}}$ in algae from the cold, lowlight treatment decreased (Tukey post hoc, rmANOVA, $\mathrm{p}=0.005$ ); it then remained unchanged over the next $4 \mathrm{~h}$ (Tukey post hoc, rmANOVA, $\mathrm{p}=0.99$ ). Between $2-4 \mathrm{~h}$, high light affected $\mathrm{E}_{\mathrm{k}}$ with a decrease in the cold treatment (Tukey post hoc, rmANOVA, $\mathrm{p}=0.024$ ) and an increase in the warm treatment (Tukey post hoc, rmANOVA, $\mathrm{p}=0.033$ ). $\mathrm{E}_{\mathrm{k}}$ in the control treatment (L16) did not change over time $(0.078 \geq \mathrm{p} \leq 1.00)$. At the end of the experiment, only the $E_{k}$ values in the warm treatments were different (greater) than in the control (L16) (Tukey post hoc, two-way ANOVA, $\mathrm{p}<0.001)$. 

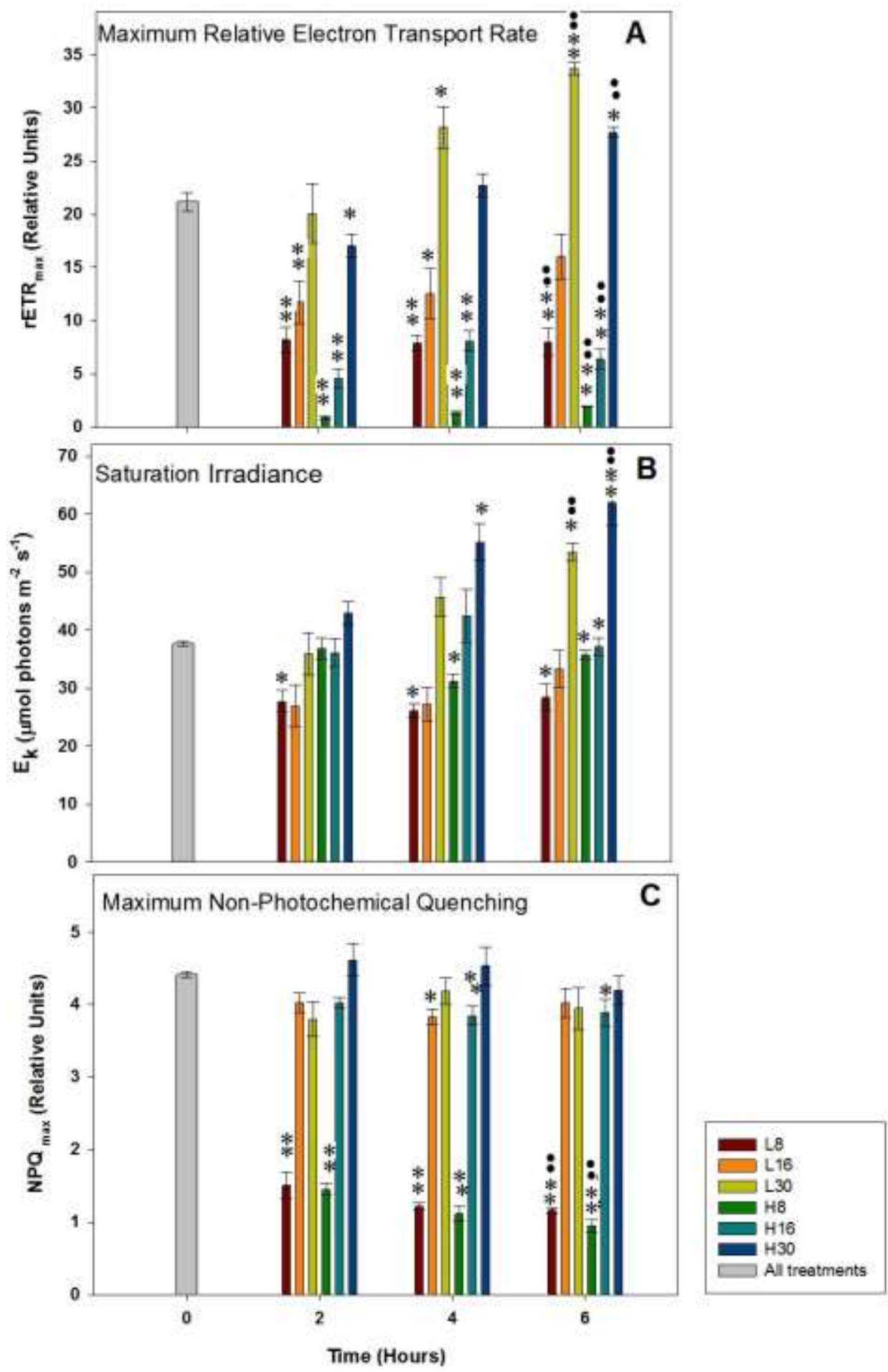

Figure 3.9: Parameters of the rapid light curves (RLC). A) $\mathrm{rETR}_{\max }$; $B$ ) $E_{k}$ and; C) $\mathrm{NPQ}_{\max }$ in all six treatments after $0,2,4$ and $6 \mathrm{~h}$ (means $\pm \mathrm{SE}, \mathrm{n}=4$ ). All parameters were analysed with rmANOVA. Notations indicate differences from the initial value (Tukey post hoc, rmANOVA, $* \mathrm{p}<0.05$ and $* \mathrm{p}<$ 0.001) or where there was a difference in measurements between treatments and the control (L16) after 6 $\mathrm{h}$ (Tukey post hoc, two-way ANOVA $\bullet \mathrm{p}<0.05$ and $\mathbf{:}_{\mathrm{p}}<0.001$ ). Note the difference in scales on the y-axis. 

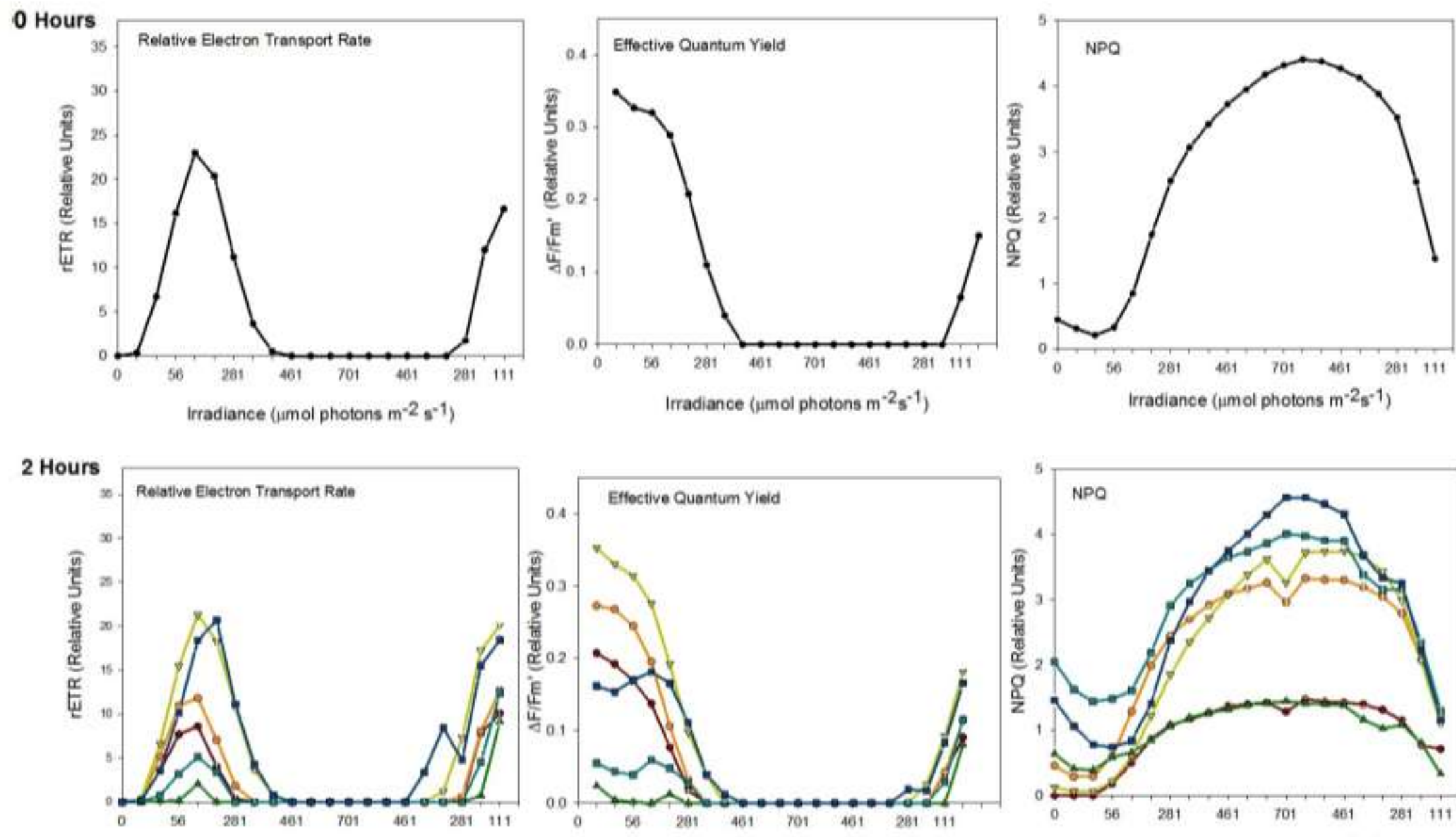

Irradiance (umol photons $\mathrm{m}^{-2} \mathrm{~s}^{-1}$ )
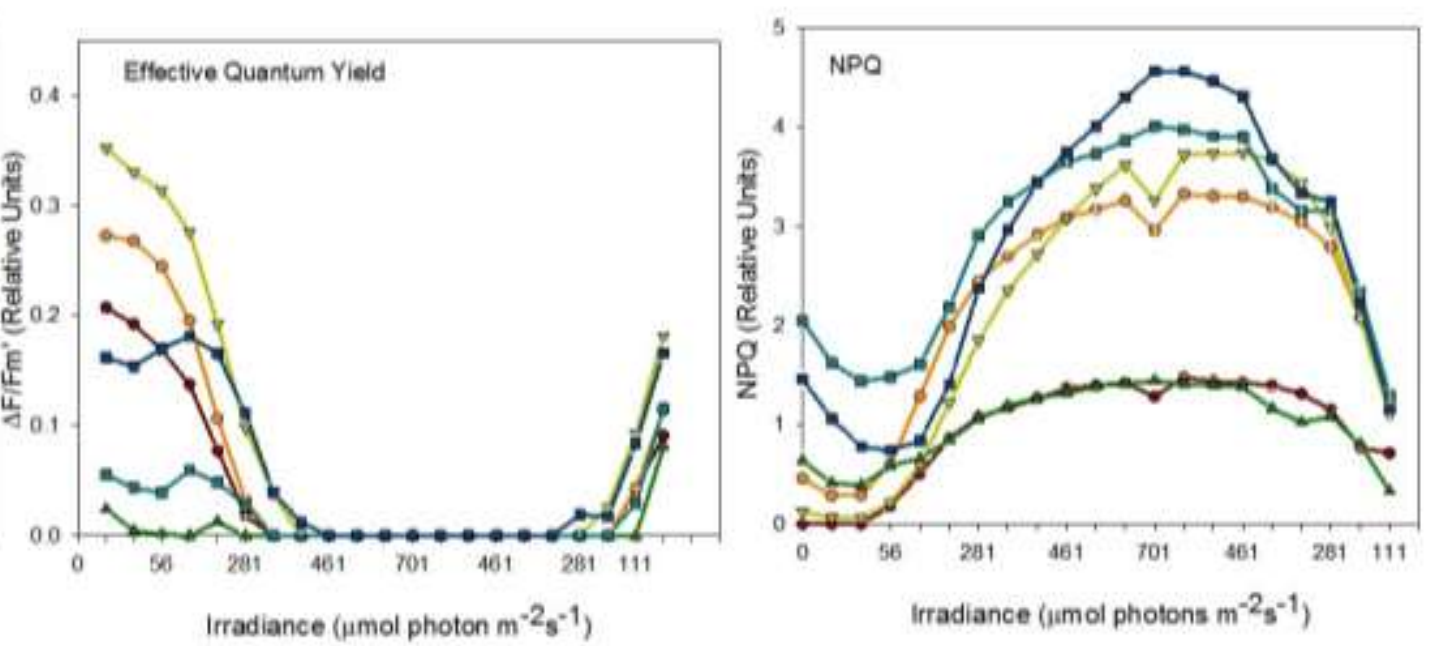

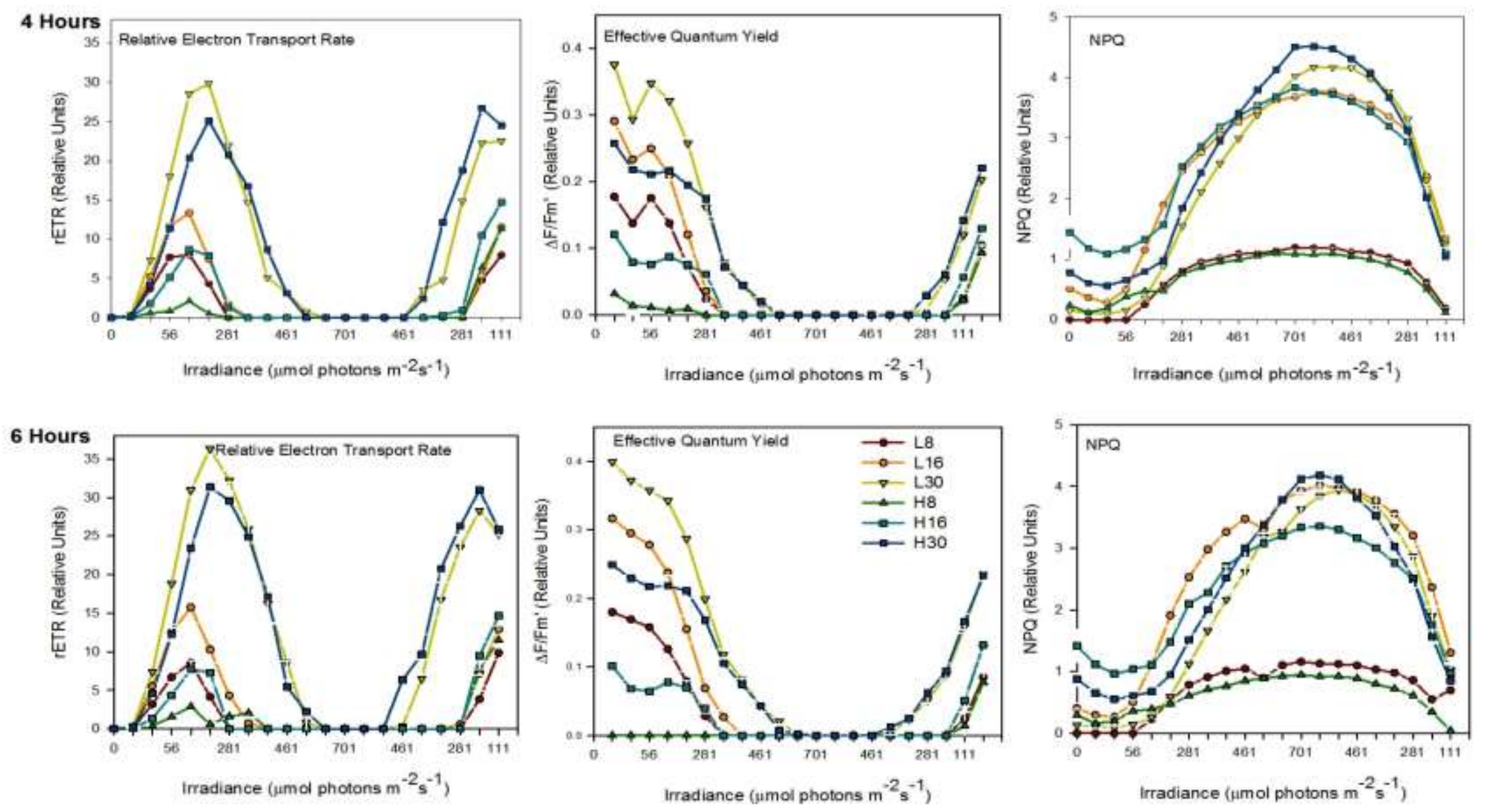

Figure 3.10: Rapid light curve parameters measured were relative electron transport rates ( rETR), effective quantum yield ( $\triangle \mathrm{F} / \mathrm{Fm}$ ') and non-photochemical quenching (NPQ) for all treatments at $0,2,4$ and $6 \mathrm{~h}$ in the experiment (Mean, $\mathrm{n}=4)$. The six treatments were low-light $8^{\circ} \mathrm{C}(\mathrm{L} 8)$; low-light, $16^{\circ} \mathrm{C}(\mathrm{L} 16)$; low-light $30{ }^{\circ} \mathrm{C}(\mathrm{L} 30)$; high-light, $8{ }^{\circ} \mathrm{C}$; high-light, $16{ }^{\circ} \mathrm{C}(\mathrm{H} 16)$ and; highlight, $30^{\circ} \mathrm{C}(\mathrm{H} 30)$. Each irradiance level was maintained for sixty seconds. 


\subsubsection{Maximum non-photochemical quenching $\left(\mathrm{NPQ}_{\max }\right)$}

Changes in $\mathrm{NPQ}_{\max }$ were affected in the first $2 \mathrm{~h}$ by temperature (rmANOVA, $\mathrm{p}<0.001$ ), with low temperature having the greatest effect under both light irradiances, where $\mathrm{NPQ}_{\max }$ decreased from the initial measurement by approximately $67 \%$ in both cold treatments (Tukey post hoc, rmANOVA, $\mathrm{p}<0.001$ ) (Figure 3.9). $\mathrm{NPQ}_{\max }$ in the cold, high-light treatment continued to decrease over the next $4 \mathrm{~h}$ (Tukey post hoc, rmANOVA, $\mathrm{p}=0.005$ ) whereas it stabilised in the cold, low-light treatment (Tukey post hoc, rmANOVA, $\mathrm{p}=0.115$ ). There was a decrease in $\mathrm{NPQ}_{\max }$ in both $16{ }^{\circ} \mathrm{C}$ treatments by $4 \mathrm{~h}$, but by $6 \mathrm{~h}$ only $\mathrm{NPQ}_{\max }$ in the high-light, moderate treatment was less than the initial measurement (Tukey post hoc, rmANOVA, $\mathrm{p}=0.04$ ). At the end of the experiment, $\mathrm{NPQ}_{\max }$ was less than the control (L16) in both cold treatments (Tukey post hoc, two-way ANOVA, $\mathrm{p}<0.001)$ whereas all the other treatments were the same.

\subsubsection{Rapid light curve parameters: $\mathrm{rETR}, \Delta \mathrm{F} / \mathrm{F}_{\mathrm{m}}$ ' and $\mathrm{NPQ}$}

Measurements were also made to compare the trends between relative electron transport (rETR), effective quantum yield $\left(\Delta \mathrm{F} / \mathrm{F}_{\mathrm{m}}{ }^{\prime}\right)$ and non-photochemical quenching (NPQ) parameter profiles throughout the RLC as actinic light increased and then decreased (Figure 3.10). The NPQ measurements at 2, 4 and $6 \mathrm{~h}$ showed that four treatments (low and high light at 16 and $30{ }^{\circ} \mathrm{C}$ ) had similar profiles to the profile at time 0 . The NPQ profiles in the cold treatments were considerably lower at $2 \mathrm{~h}$ than at $0 \mathrm{~h}$, with a decrease of the maximum measurement by $68 \%$. This profile then remained the same at the next two time-points. In the 16 and $30^{\circ} \mathrm{C}$ treatments, at all four time-points, NPQ increased with irradiance, but then relaxed rapidly as the irradiance decreased. $\Delta \mathrm{F} / \mathrm{F}_{\mathrm{m}}$, on the other hand, decreased to zero as irradiance increased and recovered once irradiance had dropped below $200 \mu \mathrm{mol}$ photons $\mathrm{m}^{-2} \mathrm{~s}^{-1}$ in the 8 and $16{ }^{\circ} \mathrm{C}$ treatments. In the warm treatments, recovery occurred at increasingly higher irradiances at 4 and $6 \mathrm{~h}$. rETR increased over time in both of the warm treatments, but more so under low light. Over the four time-points in the experiment, there was also a decrease in the length of time when rETR ceased at higher irradiances in the 
warm treatments. In the other four treatments there was an initial drop in the first peak of the rETR profiles, after which they remained constant.

\section{$\underline{\text { 3.4 Discussion }}$}

The main finding of this study is that Symbiodinium cells within A. aureoradiata are very effective in protecting themselves against photo-damage by activating an efficient NPQ system and down-regulating the quantum efficiency of PSII under different irradiances and at different temperatures. Temperate anemones are known to depend less on the photosynthetic abilities of their symbionts than those in tropical hosts (Muller-Parker and Davy 2001). As these symbionts had negligible photosynthetic activity measured, this would appear to be the case in A. aureoradiata too, though there may have been a different process occurring that was not measurable in the methods used.

\subsubsection{Down-regulation}

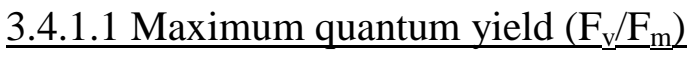

Down-regulation of photosystem II to protect against any damage by light photons can be determined by comparing measurements of maximum quantum yield, or photosynthetic efficiency $\left(\mathrm{F}_{\mathrm{v}} / \mathrm{F}_{\mathrm{m}}\right)$ (Krause 1988, Brown et al. 1999, Muller et al. 2001, Fisher et al. 2011). $\mathrm{F}_{\mathrm{v}} / \mathrm{F}_{\mathrm{m}}$ was affected more by cold temperature than by warm conditions in this study and was considerably lower, especially under high light, than $F_{v} / F_{m}$ in the other two temperatures at the 2-hour time-point. Lower quantum yield could indicate that the Symbiodinium cells were either under stress or that the photosystems had down-regulated. Calculations for the decreased $F_{v} / F_{m}$ were made using the minimal fluorescence yield $\left(F_{o}\right)$ measurements, which were much lower than those from the start. This drop in $F_{o}$ is an indication of energy quenching by $\mathrm{qE}$ (Muller et al. 2001) or an uncoupling of energy transfer by a phase shift occurring in the thylakoid membrane (Diaz-Almeyda et al. 2011), both photoprotective mechanisms. If there had been a rise in $\mathrm{F}_{\mathrm{o}}$ measurements instead, as maximum fluorescence yield $\left(\mathrm{F}_{\mathrm{m}}\right)$ and quantum yield dropped, then this would have indicated damage occurring to the PSII reaction centres (Krause 1988, Muller et al. 2001, 
Schreiber and Klughammer 2008). Therefore, down-regulation seems likely to have occurred in the photosystems in the algae from the cold treatments. Down-regulation was also noted by decreased $F_{v} / F_{m}$ and $F_{o}$ in the high-light, warm treatment after 2 hours, though this was not apparent by the end of the experiment as $F_{v} / F_{m}$ had returned to initial levels.

Though maximum quantum yield dropped considerably between $0-2$ hours in the $8{ }^{\circ} \mathrm{C}$ treatments and gradually continued to drop over time, there was only a small drop in yield in the other two temperature treatments by 2 hours. The yield in the $16^{\circ} \mathrm{C}$ treatments then remained steady over the next 4 hours, while this same parameter increased steadily in the $30{ }^{\circ} \mathrm{C}$ treatments to such an extent that $\mathrm{F}_{\mathrm{v}} / \mathrm{F}_{\mathrm{m}}$ in the low-light, $30{ }^{\circ} \mathrm{C}$ treatment was higher than the value measured at the start (0.4685 compared to 0.4516). This suggests that, though the cell counts had dropped by the end of the experiment, the remaining Symbiodinium cells were not physiologically compromised (Jones et al. 2000).

\subsubsection{Non-photochemical quenching (NPQ) and the thylakoid membrane}

Most protection by non-photochemical quenching (NPQ) occurred under high-light conditions at all temperatures tested. qN was rapidly induced and then stabilized as, simultaneously, effective PSII quantum yield decreased. The quick response by NPQ could prevent photoinhibition, while the drop in effective PSII quantum yield suggests the downregulation of the photosystems. Down-regulation, and not damage, is also suggested by the rapid light curves recovering for both the cold treatments, as the measurements at the end of the RLCs indicated rapid increase in electron transport rate (ETR) and effective PSII quantum yield $\left(\Delta \mathrm{F} / \mathrm{F}_{\mathrm{m}}{ }^{\prime}\right)$, as the irradiance was lowered, suggesting that these Symbiodinium cells have adapted to rapidly changing conditions in their habitat. In most of the treatments, $\mathrm{qN}$ and $\Delta \mathrm{F} / \mathrm{F}_{\mathrm{m}}$, mirrored each other and almost added up to 1 , indicating that the use of light photons in photochemical quenching and non-photochemical quenching was well balanced, and protected the photosystem. The only treatment in which photoinhibitory damage was suspected, rather than down-regulation, was the high-light, $30{ }^{\circ} \mathrm{C}$ treatment from 4 hours onwards. At 4 and 6 hours, maximum quantum yield remained as high as at 
the start but $\Delta \mathrm{F} / \mathrm{F}_{\mathrm{m}}$ ' dropped to zero once the actinic light was on. At the same time, $\mathrm{qN}$ was neither induced nor stabilized as rapidly as the other parameters. This suggests that there may have been free electrons in the system which could have led to the formation of destructive reactive oxygen species.

Resilience to temperature and light-stress by increased photoprotection in these Symbiodinium cells could be due to the composition of the thylakoid membranes in the chloroplasts. Thermally-damaged membranes cause uncoupling of energy transfer, but do not affect the water-splitting capabilities of the chloroplast, leading to the presence of excessive oxygen (Tchernov et al. 2004). These authors analysed the lipid composition of various Symbiodinium types and found that sensitivity to thermal damage was determined by the saturation of the lipids within the membrane, with thermally-tolerant types having significantly lower concentrations of polyunsaturated fatty acids. Similarly, in two Symbiodinium clade A types tested, rigidity of the membranes due to cold could be reduced by increasing the levels of polyunsaturated fatty acids (Diaz-Almeyda et al. 2011). These authors found that the melting curve of thylakoid membranes is type-specific, even within Symbiodinium clades. Fluidity of the membrane allows dissociation of the pigments within the antenna complex from PSII to PSI facilitating dissipation of energy as heat, which is more evident in Symbiodinium in clade A (Reynolds et al. 2008). Another reason that thylakoid lipid content could affect resilience is that lipids within the membrane help to remove oxygen from PSII (Kern and Guskov 2011). Clumping together of the lipids allows rapid diffusion of oxygen across the membrane and away from the reaction centre region, so reducing oxidative stress.

Measurement of instantaneous fluorescence yield $\left(\mathrm{F}_{\mathrm{t}}\right)$ allows an assessment of the integrity and thermostability of the thylakoid membrane by monitoring the reaction of chlorophyll fluorescence to actinic light (Hill et al. 2009). Thylakoid integrity is inferred as chlorophyll pigments are embedded within the membrane. This parameter was highest in the warmer temperatures at both light levels. Temperature may have affected the fluidity of the bi-lipid layers, which may then have affected the plasticity and resilience to the higher temperature by facilitating the repositioning of chlorophyll and carotenoid pigments (Tchernov et al. 
2004). Indeed, this mechanism could have contributed to the increase in effective PSII quantum yield in the low-light, $30^{\circ} \mathrm{C}$ treatment, and the eventual recovery in the high-light, $30{ }^{\circ} \mathrm{C}$ treatment. On the other hand, the decrease in $\mathrm{qN}$ in both $30^{\circ} \mathrm{C}$ treatments could have been caused by the change in the thylakoid membrane affecting the generation of the transthylakoid $\Delta \mathrm{pH}$, and thereby diminishing the activation of the xanthophyll cycle in the $\mathrm{qE}$ component of NPQ. This would have decreased the loss of photon energy by heat dissipation. The cold, however, could have affected the thylakoid membranes by reducing the fluidity of the bi-lipid layers, thereby leading to the uncoupling of the electron transport chain, as can be verified by the rETR profiles. Unlike Hill et al. (2009), who found that membrane sensitivity was temperature dependent but light independent, in this experiment $F_{t}$ was continuously higher in low light than in high light at all temperatures, and effective PSII quantum yield increased gradually over time for all low-light treatments. This may be due to adaptation by the Symbiodinium cells to low-light environments as A. aureoradiata are found in cracks on the rocky shore and buried on the mudflat, and also adaptation to the environment within the thick tissue of the anemone.

The induction of NPQ in the high temperature and high light treatments in this study was expected, but the measurement of $\mathrm{qN}$ indicated that there was almost as much NPQ being induced in both light irradiances in the cold temperature treatments too. Only a few studies have been carried out on the stressful effects of cold temperature on Symbiodinium photosystems (e.g. Thornhill et al. 2008, Kemp et al. 2011). Colder than normal conditions have been shown to cause bleaching in corals (Saxby et al. 2003) and photoinhibition in Symbiodinium cells (Saxby et al. 2003, Wicks et al. 2010a). Cnidarian-Symbiodinium partnerships at temperate latitudes must have adapted over time, and are able to acclimate to seasonal and daily changes, to survive in colder conditions. Higher plants in the cold are thought to keep $\mathrm{Q}_{\mathrm{A}}$, the primary quinone electron acceptor, more oxidized so that excessive light photons are unable to move into the photosystems (Huner et al. 1998, Bravo et al. 2007), and plants from the Antarctic and Andes have developed fast action NPQ to dissipate light energy away from the photosystems (Bravo et al. 2007). Likewise, dinoflagellates in polar regions protect their photosystems by having a higher ratio of 
polyunsaturated fatty acids in their thylakoid membranes to maintain membrane fluidity in the cold (reviewed in Morgan-Kiss et al. 2006). This would aid energy dissipation by state transitioning of the chloroplast pigments. Thornhill et al. (2008) hypothesised that loss of thylakoid membrane fluidity decreased photosynthetic efficiency in cold-stressed Symbiodinium in their study. Conversely, it is also thought that Symbiodinium cells become more heat tolerant with higher lipid-saturation levels to maintain thylakoid membrane integrity at higher temperatures (Tchernov et al. 2004). For temperate Symbiodinium cells to survive, in the cold of winter and the heat of summer, changes in the ratio of lipid types in the membrane throughout the year may be necessary. However, this study with low and high temperature treatments was only carried out over 6 hours. Therefore, synthesis of new thylakoid lipids would have had to be completed in a very short time span and the possibility for this happening in Symbiodinium is unlikely (Tchernov et al. 2004). If synthesis of lipids is unlikely, then the Symbiodinium cells in my study would have had an increase of the $\mathrm{qE}$ component of the NPQ mechanism in both the colder and warmer treatments and not the $\mathrm{qT}$, state transitioning component. Additional study on the ratio of the different NPQ components is needed to verify this.

\subsubsection{Photosynthetic efficiency}

High light decreased photochemical efficiency compared to low light at every temperature tested, with photosynthesis eventually ceasing in this study. Similarly, in previous studies, high irradiance also caused a decline in effective PSII quantum yield when corals were exposed to both high and low temperatures (Saxby et al. 2003, Wicks et al. 2010a). Photosynthesis may be still be occurring in the Symbiodinium cells tested here, but via a different method within the chloroplast. The Symbiodinium cells in this study were identified as types from clade A, and clade A Symbiodinium types have been found to be able to upregulate PSII-independent cyclic electron transport (CET) that transfers energy around PSII to PSI, maintaining ATP synthesis and therefore conversion of carbon from its inorganic to organic form, even when PSII is inactivated (Reynolds et al. 2008). These authors found that most members of clade A demonstrated alternative methods of photosynthetic electron transport whereas the other Symbiodinium clades did not. This 
alternative CET is an important pathway for amplifying NPQ in plants and may have a similar function in Symbiodinium dinoflagellates (Munekage et al. 2004, Warner and BerryLowe 2006). Clade A is the most ancestral of the Symbiodinium genera (LaJeunesse 2001) and has retained the alternative CET and the ability to dissociate pigments in the antennae complex, whereas the other clades have diverged from these physiological traits. Retaining these traits may explain why clade $\mathrm{A}$ is found in hosts that primarily inhabit shallow, highlight habitats (Finney et al. 2010). Further discussion on alternative CET is carried out in Chapter 4.

It was also interesting to note that the irradiance where the photosystems reached saturation point $\left(E_{k}\right)$ was so low in the rapid light curves. $E_{k}$ measurements indicate the irradiance at which the energy from absorbed light photons is transferred to NPQ for dissipation (Hill et al. 2004). The highest $E_{k}$ in the rapid light curve of the present study of $61.05 \mu$ mol photons $\mathrm{m}^{-2} \mathrm{~s}^{-1}$ was well below the irradiance in the high-light treatments of $400 \mu$ mols photons $\mathrm{m}^{-2}$ $\mathrm{s}^{-1}$, and less than the low-light treatment $\left(100 \mu\right.$ mols photons $\left.\mathrm{m}^{-2} \mathrm{~s}^{-1}\right) . \mathrm{E}_{\mathrm{k}}$ was also much lower than the radiance experienced on the mud flats from where the anemones were collected (summer maximum, $1614 \mu \mathrm{mol}$ photons $\mathrm{m}^{-2} \mathrm{~s}^{-1}$; winter maximum, $795 \mu \mathrm{mol}$ photons $\mathrm{m}^{-2} \mathrm{~s}^{-1}$ (Gibbons 2008)). This could be due to the Symbiodinium cells within the anemones already being physiologically acclimatised to the low light conditions whilst being in the laboratory $\left(100 \mu\right.$ mols photons $\left.\mathrm{m}^{-2} \mathrm{~s}^{-1}\right)$ or when covered by mud in the field, or it could be due to the Symbiodinium cells being acclimatised to the shading caused by their endosymbiotic habitat (Bhagooli and Hidaka 2003)

It could also be that the Symbiodinium cells functioned very differently than they would have done, had they been in their normal environment within the host. The host tissue would have protected them by reflecting and reducing light (Banaszak et al. 2000). Also the host cell cytoplasm would have provided the Symbiodinium cells with different nutrient and ionic concentrations than seawater (Seibt and Schlichter 2001), which could have induced a degree of stress. For example the photochemical efficiency of Symbiodinium cells isolated from four different hosts species decreased significantly and recovered more slowly than when the same Symbiodinium types were being tested within their hosts (Bhagooli and 
Hidaka 2003). Similar to the results in my study, the 2003 study found that even exposure to low light $\left(70 \mu \mathrm{mol}\right.$ photons $\left.\mathrm{m}^{-2} \mathrm{~s}^{-1}\right)$ caused a significant drop in photosynthetic efficiency at both control and higher temperatures. As the irradiance was increased, yield almost dropped to zero, which was similar to the zero quantum yield measured in the current study under higher irradiance $\left(400 \mu \mathrm{mol}\right.$ photons $\left.\mathrm{m}^{-2} \mathrm{~s}^{-1}\right)$. The present study tested photoprotection under a sudden change of light intensity and temperature, leading to shortterm stress effects. On a daily basis, irradiance levels could change as rapidly as they did in the experiment but, in reality, temperature would change gradually through the day and through the seasons. Future experiments following the same PAM procedures from this study, but working with the holobiont would be valuable to determine if residing in the anemone affects the photosynthetic outcomes significantly.

There have not been many studies of light and temperature stress in high-latitude types of Symbiodinium. Wicks et al. (2010) carried out studies on the high latitude coral Pocillopora damicornis at Lord Howe Island. They placed nubbins of corals into six different temperature and light combinations and conducted measurements every three hours for three days using a Diving-PAM. They measured effective quantum yields $\left(\Delta F / F_{m}{ }^{\prime}\right)$ and fast induction curves (FIC), and found that the Symbiodinium cells in the corals were more tolerant to a drop in temperature $\left(-3^{\circ} \mathrm{C}\right)$ from the control compared to an equal rise in temperature above the control, though there was a drop in $\Delta \mathrm{F} / \mathrm{F}_{\mathrm{m}}$ ' at both temperatures, and more so in the high-light treatments. NPQ was also greater in high-light than low-light treatments. Wicks and co-workers concluded from this that the Symbiodinium cells were specialized to function in the cold as they originate from relatively high-latitude waters. The induction curves presented in this study also showed more deleterious effects under high light compared to low light at the same temperature. The main difference between the two studies was that, at the higher temperature, but in low light, the Symbiodinium cells in my study appeared to increase in photosynthetic health; NPQ decreased and $\Delta \mathrm{F} / \mathrm{F}_{\mathrm{m}}$ ' increased. Could this be because the anemones in New Zealand have had to also specialize to being in warm habitats such as rockpools or shallow pools on mudflats, whereas the shallowest the corals in Wicks et al.'s study were found was in 2-3 m depth in the sea? 
This study has resolved some of the objectives set out at the start. The Symbiodinium cells do have strong photoprotective abilities, and can down-regulate the quantum efficiency of PSII quickly and effectively but do this at high and low temperatures. However, to learn how they have photoacclimated will need further studies. These could include molecular analysis to establish if genes are present and upregulated for chloroplast pigment and thylakoid lipid synthesis. The thylakoid membrane can be examined by high-performance liquid chromatography (HPLC) to measure the ratio of different pigment and lipid types present. Also measurement of the three components of NPQ will enable an assessment of which provided the most photoprotection. 


\section{Chapter 4}

\section{General Discussion}

This study examined the molecular identity of the Symbiodinium types within the local symbiotic anemone, Anthopleura aureoradiata, and how biogeographical isolation, latitudinal distribution and local habitat could affect symbiont diversity. A comparison was also made with the Symbiodinium types found within four species of anemones from Europe to identify differences, or similarities, between types from similar latitudes. As predicted, diversity was low but, contrary to expectations, the isolation of New Zealand does not seem to have led to endemicity of these symbionts, as there was a marked similarity between the Symbiodinum types from New Zealand, southern England, Brittany (France) and the Mediterranean. The Symbiodinium types from all the anemones in the study were identified as being similar or identical to ITS2 types Symbiodinium Amed, ("Amed") and Mediterranean clade A ("Med clade A"). These two types were both originally sourced and identified from the Mediterranean Sea (Hunter et al. 2007, Meron et al. 2012)

The effects of different temperature and light regimes on the photophysiology of the $A$. aureoradiata-Symbiodinium symbiosis were also examined, to better understand how this association remains functional under a wide range of environmental conditions. As predicted, the photoprotective capacity of the non-photochemical quenching mechanism was quickly and effectively utilized by the algal cells but, surprisingly, there was little or no photosynthesis measured, except under low light at 16 and $30{ }^{\circ} \mathrm{C}$. These Symbiodinium cells effectively protect their photosystems by converting photon energy into heat and not into chemical energy. 
Two main questions arose from the outcomes of the study and will be discussed further in this section.

1. How did Med clade A and Amed Symbiodinium get to both hemispheres, and how long ago did the populations separate?

2. What is it about clade A photophysiology that makes it so suitable for temperate regions?

\subsection{Population distribution - How and when?}

\subsubsection{Ancient Origins}

There is evidence to suggest that the mutualistic relationship between anemone-like coral ancestors and Symbiodinium-like dinoflagellates began in the late Triassic period, about 235 million years ago (MYA) (Stanley and Fautin 2001, Muscatine et al. 2005, Stanley 2006). Estimations from molecular clock deductions suggest that Symbiodinium clade A originated from the Paleocene time, 65-50 MYA, and went through diversification within the clade and divergence into the other clades throughout the next 35-50 million years (Tchernov et al. 2004, Pochon et al. 2006). Throughout this time the position of the world's landmasses was very different to today (Figure 4.1).

There was a continuous band of sea around the northern hemisphere and open water between what was to become North and South America. Ocean currents flowed differently than they do today, and the temperature of the oceans was less differentiated between the furthest latitudes, as the establishment of cold water domains at the poles did not occur until 16 MYA ( \pm 8 MYA) (Darling et al. 2000). Closure of the Mediterranean Sea to the east occurred about 20 MYA (Hrbek and Meyer 2003) and the final closure of the Isthmus of Panama occurred approximately 3.5 MYA (Coates et al. 1992), stopping direct links between the Atlantic Ocean and the Pacific and Indian Oceans. 


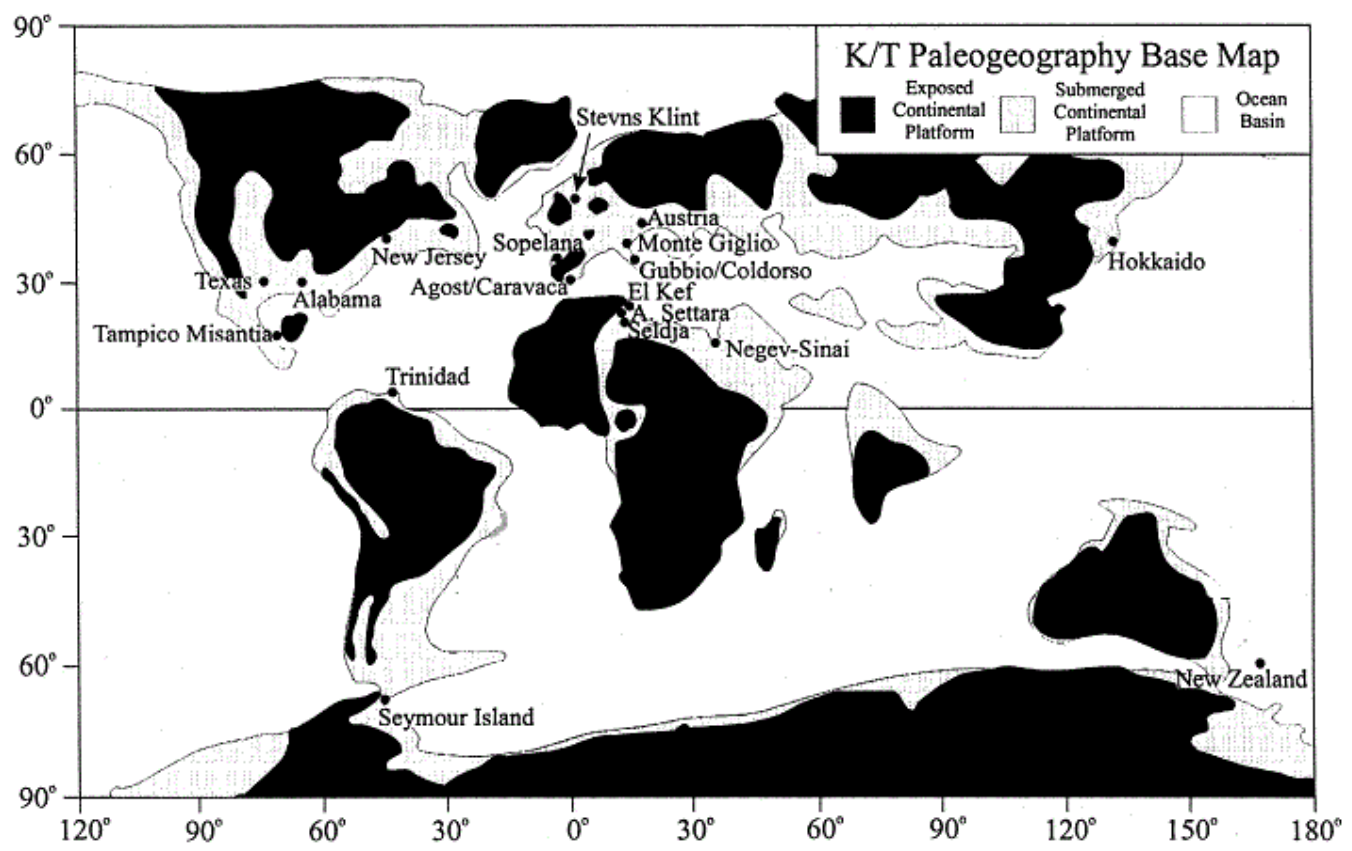

Figure 4.1: Paleogeographic map of 65 MYA showing the open water linkage across the northern hemisphere, and a link between what will become the Atlantic and Pacific Oceans where the Isthmus of Panama will form. (Culver 2003).

There was a continuous band of sea around the northern hemisphere and open water between what was to become North and South America. Ocean currents flowed differently than they do today and the temperature of the oceans was less differentiated between the furthest latitudes, as the establishment of cold water domains at the poles did not occur until 16 MYA ( \pm 8 MYA) (Darling et al. 2000). Closure of the Mediterranean Sea to the east occurred about 20 MYA (Hrbek and Meyer 2003) and the final closure of the Isthmus of Panama occurred approximately 3.5 MYA (Coates et al. 1992), stopping direct links between the Atlantic Ocean and the Pacific and Indian Oceans.

Establishment of the distribution for Med clade A and Amed Symbiodinium in New Zealand, England and what was to be the Mediterranean Sea, could have occurred before the waterways closed 3.5 MYA. The length of time from then to now and the apparent isolation of these populations from each other would suggest that genetic divergence would have occurred, as mutations over time would be expected in each population. However, studies of molecular data indicate that divergence can be delayed for millions of years 
(Gould and Eldridge 1977, Soltis et al. 2002, LaJeunesse 2005) and separated populations can remain undifferentiated due to limited sexual reproduction (LaJeunesse 2005). For instance, ribosomal sequences in the ancestral "types" C1 and C3 in the Atlantic-Caribbean and Indo-Pacific regions have remained unchanged since at least the time of the Isthmus of Panama closure (LaJeunesse 2005), which may also be the case with northern and southern hemisphere populations of Med clade A and Amed Symbiodinium.

\subsubsection{Vectors of connectivity}

Bipolar distributions of the same species can be found in many organisms and many planktonic groups (Darling et al. 2000), and whole microplankton communities in the north and south are essentially alike, even though they have been separated since the Miocene (20MYA) by the tropical zone (Taylor et al. 2008). Northern and southern hemisphere dinoflagellate communities are virtually identical within similar latitudinal and climatic zones (Taylor et al. 2008). Connectivity between the hemispheres may have occurred in relatively recent times and may still be occurring today. However, for genetic exchange to occur between the high southern and northern latitudes, warm equatorial seas have to be crossed. Darling et al. (2000) suggested that connection between subpolar populations of planktonic foraminiferans is made possible by individuals moving with the cold boundary currents up the continents and crossing the tropics by submergence below the deep, cold thermocline. Antarctic and Arctic population of the ciliate Euplotes nobilii are also thought to migrate this way (Giuseppe et al. 2011). However, this does appear to be an unlikely route for photosynthesising dinoflagellates. Nonetheless, a study in 2003 found two strains of a species of a photosynthesising dinoflagellate, Polarella glacialis, in the Antarctic Ross Sea and Canadian Arctic waters (Montresor et al. 2003). These authors hypothesised that the dinoflagellate is able to cross tropical waters in the encysted resting stage of its lifecycle. Also of note, a new paper presents evidence of isolated symbiotic Symbiodinium cells feeding heterotropically on bacteria and micro-algae (Jeong et al. 2012), and so not relying on the sun or a host as a food source. This ability would facilitate the migration of these dinoflagellates. 
To cross great distances, Symbiodinium cells would have to be able to live independently in seawater and travel either in ocean currents or the ballast water of ships and boats. Many Symbiodinium types must have free-living stages as, in the majority of cases, host cnidarians acquire their symbionts via horizontal transmission. The presence of free-living Symbiodinium cells is more common than once thought (Stat et al. 2008a, Pochon et al. 2010), especially in sediment (Gou et al. 2003, Hirose et al. 2008, Takabayashi et al. 2012). Gou et al. (2003) found Gymnodinium dinoflagellates in water samples from Jiaozhou Bay, China that they identified as being possibly the same species as G. varians from Wellington Harbour and S. californium from Santa Barbara, USA. Whether these dinoflagellates naturally have such a wide distribution or were spread via ballast water is unknown. Nevertheless, the extent to which a Symbiodinium cell can remain healthy and survive in the sea would differ from one species or type to another (LaJeunesse et al. 2009). In the current case, it is unlikely that Med clade A and Amed would be able to last long enough in the water to be able to travel such distances as they have evolved to function as endosymbionts (LaJeunesse et al. 2009). Identification of free-living and symbiotic Symbiodinium types in the same habitat has shown little overlap between the two communities which leads to the proposal that Symbiodinium types may be restricted to having either long-term free-living or endosymbiotic modes of living (Hirose et al. 2008, Pochon et al. 2010, Takabayashi et al. 2012). Moreover the endosymbionts identified in this study are known to be maternally transmitted from an adult anemone to the young, in all but one of the anemone species sampled (A. mutabilis has not been studied yet) (Bythell et al. 1997, Davy et al. 1997a, Davy and Turner 2003), and so a long free-living stage would not be envisaged. However, the lack of genetic differences encountered from the samples analysed from the north to the south of NZ suggests sharing of genes, in which case, some free-living Med clade A and Amed Symbiodinium would be required with some horizontal transmission occurring in the A.aureoradiata population, (Focioli et al. 2011).

Conversely connectivity between the northern and southern latitudes may occur by these Symbiodinium types migrating as endosymbionts within pelagic hosts, whether naturally with currents or within the ballast waters or on hulls of boats, and being passed on from 
these hosts into native animals. One of the organisms that hosts Symbiodinium clade Temperate-A is Cotylorhiza tuberculata, the Mediterranean fried egg jellyfish (Visram et al. 2006; Figure 4.2 A). Closely related species of jellyfish can be found in the Atlantic and Pacific Oceans with, for example the symbiotic jellyfish Phyllorhiza punctate (Figure 4.2 B) inhabiting New Zealand waters (Gordon 2009). It is conceivable that Med clade A and Amed may have colonized two distant regions by passing through a network of jellyfish hosts, though there is no evidence for this hypothesis.

Another consideration is that these clade A Symbiodinium types appear to be hostgeneralists in the Mediterranean and around the English coast. No other cnidarianSymbiodinium partnerships have been identified in New Zealand and it would be interesting to discover if the jellyfish Phyllorhiza punctata does host Med clade A and Amed, or if there are any other host animals along the New Zealand coastline which also harbour Symbiodinium cells of these types.

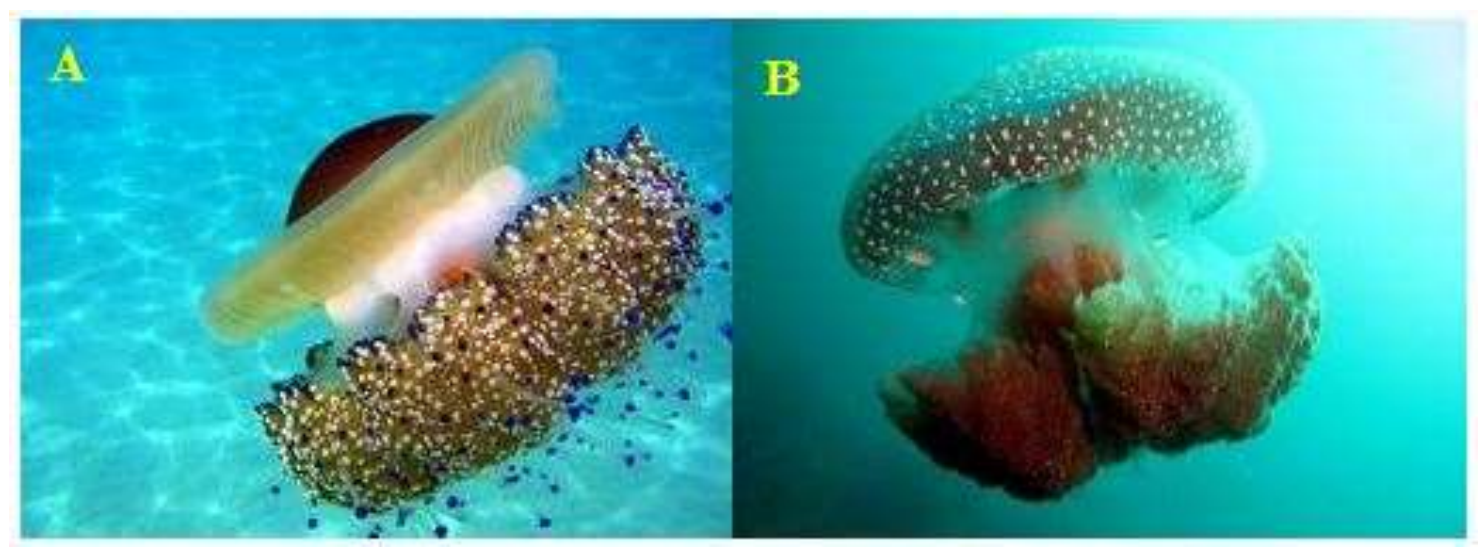

Figure 4.2: A) Cotylorhiza tuberculata, the Mediterranean fried egg jellyfish (image from http://fc09.deviantart.net/fs71/f/2010/009/r/0Cotylorhiza-tuberculata-6-149764996) and; B) Phyllorhiza punctate, the Australian spotted jellyfish (image by $\mathrm{K}$ Kalani Patterson from www.gambassa.com/public/project/2066/PoojaPatelNotableSpecies.html)

An alternative idea is that the widespread distribution of Med clade A and Amed occurred together with the anemone host. Anthopleura ballii from England sampled in this study 
hosted Med clade A, and Symbiodinium cells identified in Anthopleura japonica and Anthopleura kurogane from Korea hosted members of Symbiodinium clade A too (Rodriguez-Lanetty et al. 2000). This partnership between Anthopleura anemones and clade A Symbiodinium may be an ancient combination that took place before the landmasses parted and shifted into the geographical positions of today, whilst maternal transmission in these anemones has maintained the genetic similarities between the northern and southern distribution of these Symbiodinium types.

\subsection{What is special about clade A as a temperate symbiont?}

The species Anthopleura aureoradiata was found to host Symbiodinium Med clade A in the majority of cases with very few individuals hosting Symbiodinium Amed throughout the whole of its biogeographical range (97\% Med clade A versus 3\% Amed). Other studies have found that Symbiodinium type changes within host species with latitude (e.g. Lien et al. 2007), season (e.g. Venn et al. 2008b) and shore height (e.g. Baker 2003). However, the relationship that $A$. aureoradiata has with its symbionts appears to be stable even though the climatic conditions in New Zealand are so varied and changeable, and the habitats where this symbiosis is found are relatively diverse. Tropical cnidarian-Symbiodinium partnerships can break down with only a small change in temperature, sometimes as little as 1-2 ${ }^{\circ} \mathrm{C}$ above the norm (Hoegh-Guldberg 1999, Hughes et al. 2003), whereas temperate partnerships are more hardy as they have had to acclimate to the wide range of seasonal conditions (Verde and McCloskey 2007).

\subsubsection{Partnership persistence with minimal nutritional benefits}

Though A. aureoradiata hosts these two Symbiodinium types throughout the year, in this study it appeared that the algae were not photosynthetically productive in most of the conditions tested in the laboratory. Photosynthesis increased in warmer conditions but only when the light levels were low. High light conditions appeared to inhibit photosynthesis altogether. With such low rates of photosynthesis, the Symbiodinium cells in $A$. aureoradiata may only be contributing adequate carbon for the anemone's respiratory 
requirements during certain times of the day or year. Other studies looked at translocation of carbon by temperate algae to their animal hosts (Davy et al. 1996, Verde and McCloskey 2007, Bergschneider and Muller-Parker 2008, Gibbons 2008) and found that, in most conditions, translocation was such that heterotrophic feeding would be necessary to meet animal respiratory requirements. This was particularly true in "winter" conditions, when for example Gibbons (2008) found that there was potentially no contribution from the algae to the animal host in A. aureoradiata. This current study did not look at the contribution of carbon to the host, but concluded that photosynthesis in all treatments was so minimal that there would be very little energy gain for the host under high irradiance. However, Gibbons' (2008) work on A. aureoradiata measured oxygen production from Symbiodinium cells' photosynthetic responses in increasing quantities up to $200 \mu \mathrm{mol}$ photons $\mathrm{m}^{-2} \mathrm{~s}^{-1}$, which then remained steady as irradiance was increased to $1000 \mu \mathrm{mol}$ photons $\mathrm{m}^{-2} \mathrm{~s}^{-1}$. If oxygen electrodes can detect oxygen production by Symbiodinium cells in A. aureoradiata, suggesting effective photosynthesis, why did the PAM fluorometry method in the current study not do so?

\subsubsection{Alternative methods of making chemical energy}

Reynolds et al. (2008) used a novel method of measuring photosynthesis with a PAM fluorometer, by measuring chlorophyll $a$ fluorescence in response to serial irradiation pulses (SIP). They found that members of clade A Symbiodinium were capable of alternative photosynthetic electron-transported pathways, such as PSII-independent cyclic electron transport (CET) and chlororespiration, where oxygen serves as an alternative photosynthetic electron transport acceptor. What this means is that both CET and chlororespiration can continue ATP synthesis when PSII becomes inactivated, and therefore conversion of inorganic carbon to organic carbon continues too. They also found that PSII in clade A become inactivated quickly in high light (exposure for 100 minutes at $250 \mu \mathrm{mol}$ photons $\mathrm{m}^{-2} \mathrm{~s}^{-1}$ ) when up to half of the peridinin-chlorophyll $a$-protein (PCP) antenna complexes disassociated from PSII in a form of NPQ state transitioning. Another benefit is that chlororespiration may also decrease the probability of reactive oxygen species (ROS) formation, and the damage that can ensue, by using oxygen as an electron 
carrier. They found that all of these qualities have been lost in the other Symbiodinium clades.

What this could mean in relation to Symbiodinium types Med clade A and Amed is that they may still be supplying carbon to the animal in high light, but in a process that is not detectable by the PAM fluorometry methods used here. The points at which I measured zero for $\Delta \mathrm{F} / \mathrm{F}_{\mathrm{m}}$ ' under high light $\left(400 \mu \mathrm{mol}\right.$ photons $\left.\mathrm{m}^{-2} \mathrm{~s}^{-1}\right)$ are about when Reynolds and co-workers suggested that PSII shuts down, due to efficient NPQ, and CET and chlororespiration pathways are used instead to bypass PSII and produce ATP. On the other hand, type A2 cells were found by the authors to not have CET and chlororespiration responses. In my study, the molecular analysis of ITS2 sequences from all samples tested placed Med clade A and Amed in a sister group to clade A2 in both Maximum Likelihood and Bayesian constructed phylogenetic trees. Type A2 and these sequences together are placed as a sister group to all the other clade A types. LaJeunesse (2001) also placed A2 as a sister group to the other clade A types. Therefore, the Symbiodinium types in the current study may share the same photophysiological traits as clade A2. The serial irradiation pulses method with the PAM fluorometer would need to be used on Symbiodinium from A. aureoradiata to confirm whether alternative photosynthetic electron-transport pathways are being used.

Clade A types have also been considered to be less beneficial to their hosts than other types (Stat et al. 2008b). Stat et al. (2008) worked with freshly-isolated clades A and C Symbiodinium and found that both fixed similar amounts of carbon in filtered seawater but once incubated in a synthetic 'host factor', clade $\mathrm{C}$ fixed and released four times as much carbon as clade A, which did not have any change in the amount of carbon fixed. This suggested that a host has no influence on photosynthate release from clade A Symbiodinium, and the fact that clade A was found in more diseased corals than was clade $\mathrm{C}$, persuaded the authors to conclude that clade A algae are nutritionally less beneficial to their host than are other Symbiodinium clades. These authors theorized that clade A Symbiodinium cells have not fully evolved into mutualistic endosymbionts from their freeliving ancestors, whereas more derived clades may have attained a more mutualistic state 
over time. However, A. aureoradiata's symbionts are passed onto the next generation vertically from the mother which, according to some theories, suggests that the symbionts are more likely to be mutualistic, with a substantial degree of host-symbiont integration (Sachs and Wilcox 2006). Moreover, carbon budgets constructed by Gibbons (2008) for this sea anemone suggest that, under well-lit conditions at least, the host may derive a substantial portion (sometimes $>100 \%$ ) of its metabolic needs from its symbionts. For much of the year though, this same author demonstrated that A. aureoradiata receives much less photosynthetic carbon than it needs due to light limitation.

\subsubsection{Persistence through vertical transmission}

If there is only a moderate nutritional benefit to hosting these temperate Symbiodinium types, why has the partnership persisted across generations? It was thought that clade A Symbiodinium cells were found in cnidarian hosts predominantly located in shallow water habitats as they produced UV-absorbing mycosporine-like amino acids (MAAs), thereby protecting their host, whereas other clades did not offer this protection. (Banaszak et al. 2000, Reynolds et al. 2008, Finney et al. 2010). However, the study by Banaszak et al (2000) was done with Symbiodinium cultures; in a more recent study, it was found that most Symbiodinium clades in hospite produce MAAs and translocate these to their host (Banaszak et al. 2006). So hosting clade A Symbiodinium in shallow NZ waters would not necessarily give more protection from solar radiation than a Symbiodinium type from another clade. It may simply be the case that A. aureoradiata hosts Med clade A and Amed because there are no other types in New Zealand waters to associate with and associating with them for the few times that they are nutritionally beneficial is more cost effective than not associating with them at all. The times of year that the holobiont receives energy surplus to its requirements may give it a competitive edge over other organisms within its habitat and may go towards the production of anemone gametes (Muller-Parker and Davy 2001). As the symbionts are vertically transmitted, this would also mean increasing the

population and distribution of the Symbiodinium algae. Symbiodinium cells may be maternally transferred due to the scarcity of Symbiodinium cells in temperate seas and/or a shortage of potential hosts (e.g. A. aureoradiata is the only known host of Symbiodinium in 
New Zealand). Moreover, the stability of the partnership combined with its mode of symbiont acquisition may be the underlying reason why the holobiont has persisted and been an ecological success around New Zealand.

\section{$\underline{\text { 4.3 What can be done to further understand Symbiodinium types in clade A }}$}

Measuring the amount and ratio of different lipid types and pigments in the thylakoid membrane of the Symbiodinium chloroplast would help clarify the mechanisms of NPQ that aid the apparent resilience of the algae to thermal stress. Gas chromatography and highperformance liquid chromatography (HPLC) can be used to identify the lipid types within the membrane (Plante et al. 2011) and whether the polyunsaturated lipid content increases as latitude increases. Using the same samples, HPLC can be used to study the pigment profile of the membranes (Venn et al. 2006, Wakahama et al. 2012). The study of two Symbiodinium types in clade A by Kraemer et al. (2012) found that there was a reduction in light-harvesting pigments and an increase in the xanthophyll pool size when under thermal stress. Reducing the light-harvesting pigments would decrease the amount of photons in the photosystems, which in turn would decrease photochemical efficiency of PSII. This could have been the reason for the low to zero measurements in this study for the effective quantum yield $(\Delta \mathrm{F} / \mathrm{Fm}$ ') of samples during dark-light induction, especially those in the highlight treatments, and also for the zero $\Delta \mathrm{F} / \mathrm{Fm}$ ' for all treatments once the irradiance reached $400 \pm 50 \mu \mathrm{mol}$ photons $\mathrm{m}^{-2} \mathrm{~s}^{-1}$ in the rapid light curves. Hennige et al. (2009) also found that Symbiodinium cells exposed to high light had a decrease in their photosynthetically active pigments, peridinin and chlorophylls $c$ and $a$. Other papers also found an increase in the xanthophyll pool (diadinoxanthin and daitoxanthin) within the thylakoid membrane, which would allow for faster heat dissipation and dispersal of too much light energy in the photosystem (e.g. Venn et al. 2006, Warner and Berry-Lowe 2006, Ulstrup et al. 2008, Hennige et al. 2009, Kraemer et al. 2012).

Resilience studies would also have to include the anemone, whose behaviour and physiology could protect the symbionts. From personal observations of the anemone, it was difficult to measure fluorescence using the PAM fluorometer once the anemone had 
withdrawn its tentacles, presumably due to the animal tissue being thick enough to reduce the amount of light reaching the algae. On mudflats, the anemones positioned on the surface were seen to be open in the early morning light but would close nearer noon. This was also seen in rockpools, though anemones on the rocky shore were more plentiful within cracks in the rocks. The anemone could also protect its symbionts by storing UV-absorbing mycosporine-like amino acids (MAAs) (Shick and Dunlap 2002, Lesser and Farrell 2004) and heat-shock proteins (Fitt et al. 2009) as well as animal tissue attenuating and reflecting light away from the algal cells (Banaszak et al. 2000).

\subsection{Concluding Remarks}

Anthopleura aureoradiata from New Zealand hosts two ITS2 types of Symbiodinium from clade A, which are similar to or identical to those in the anemones sampled from the South of England and from Brittany (France). All these types most closely match ITS2 sequences of Symbiodinium Amed and Symbiodinium Mediterranean clade A (Hunter et al. 2007, Meron et al. 2012), with most harbouring Med clade A. There was no clear latitudinal, seasonal or habitat-related pattern of symbiont distribution, or any pattern with respect to whether an anemone hosted just one symbiont type or both simultaneously. Further molecular analyses using more sensitive methods, such as quantitative PCR would be able to clarify this, especially now that the Symbiodinium types have been identified and unique primers can be designed. Likewise, running PCR-RFLP on some samples would conclusively show whether the ITS2 sequences from the Symbiodinium types here could also be classified as 'phylotype Temperate A', and so be included in the same group as the northern hemisphere Symbiodinium in cnidarians studied by Bythell et al. (1997), Savage et al. (1997), Visram et al. (2006) and Forcioli et al. (2011).

Like other temperate symbiotic anemones, A. aureoradiata relies less on its symbionts' photosynthetic resources than tropical symbiotic cnidarians do (Muller-Parker and Davy 2001), though the benefits gained may give the host a competitive advantage in its habitat (Muller-Parker and Davy 2001). Resilience to temperate conditions by the partnership between A. aureoradiata and its symbionts, Med clade A and Amed, is probably due to the 
physiological benefits the symbionts, and other clade A Symbiodinium, have to photoprotect themselves against harsh and changeable conditions. Episodes of bleaching in tropical regions are occurring more frequently, with climate changes affecting temperature and weather patterns (Hoegh-Guldberg and Bruno 2010). However, climate change is happening on a global scale and affects temperate regions too. Understanding the photophysiology of cnidarian-Symbiodinium symbiosis in temperate regions, and how it responds to environmental change, will help understand whether these organisms have as gloomy an outlook as their tropical counterparts, or whether the resilience built by adaptation to variable temperate conditions has made them more likely to survive and continue into the future. Temperate regions may become a refuge for the 235 million yearold cnidarian-Symbiodinium symbiosis if warming continues at a rate too fast for tropical partnerships to acclimate, adapt and survive.

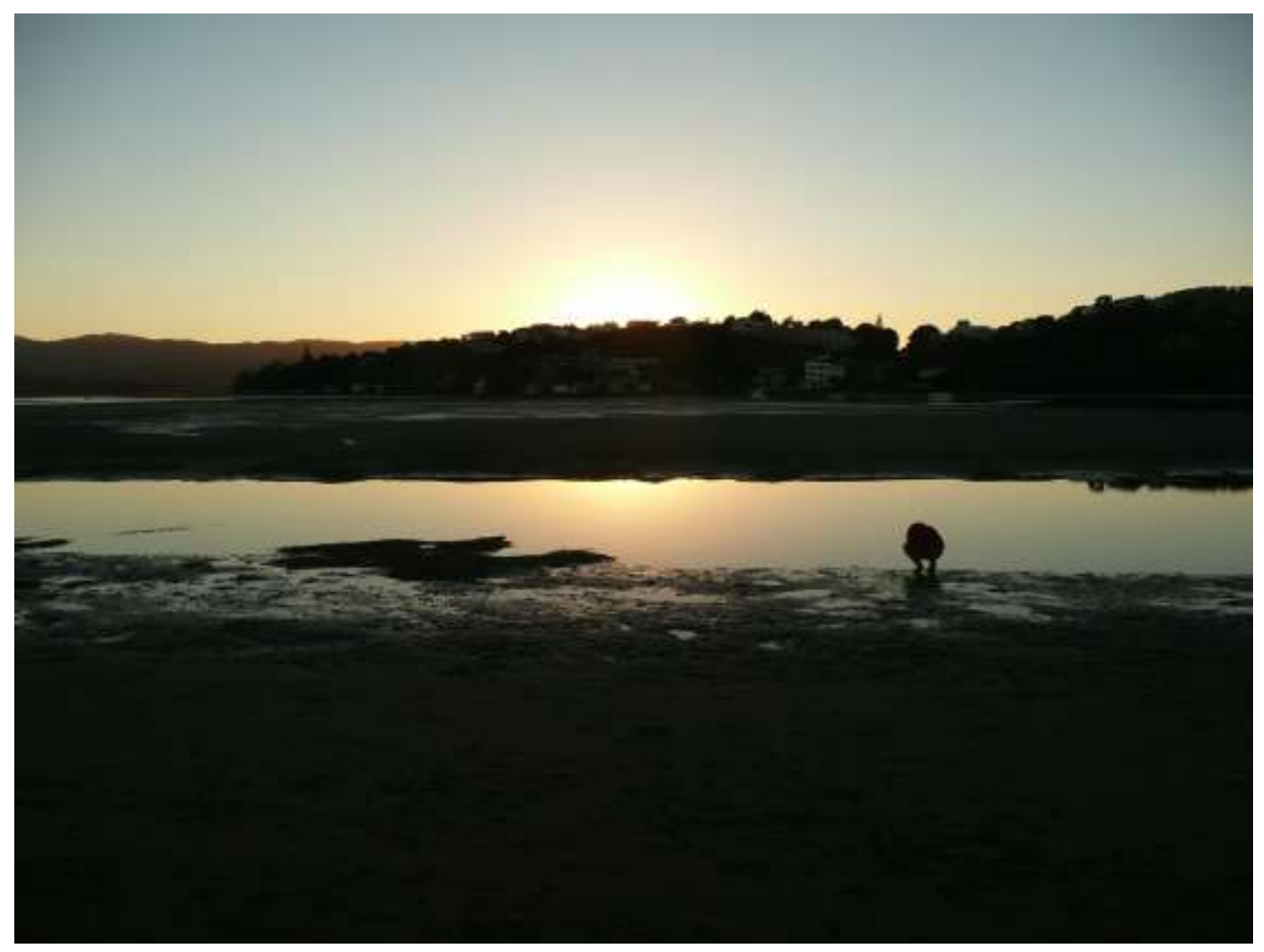

Figure 4.3: Early morning collection of anemones on the mudflats of Pauatahanui Inlet. 


\section{Appendices}

\section{APPENDIX 1 - Climatic conditions in New Zealand for 5 years prior to molecular study}

\section{A1.1 Temperature and radiance for New Zealand}
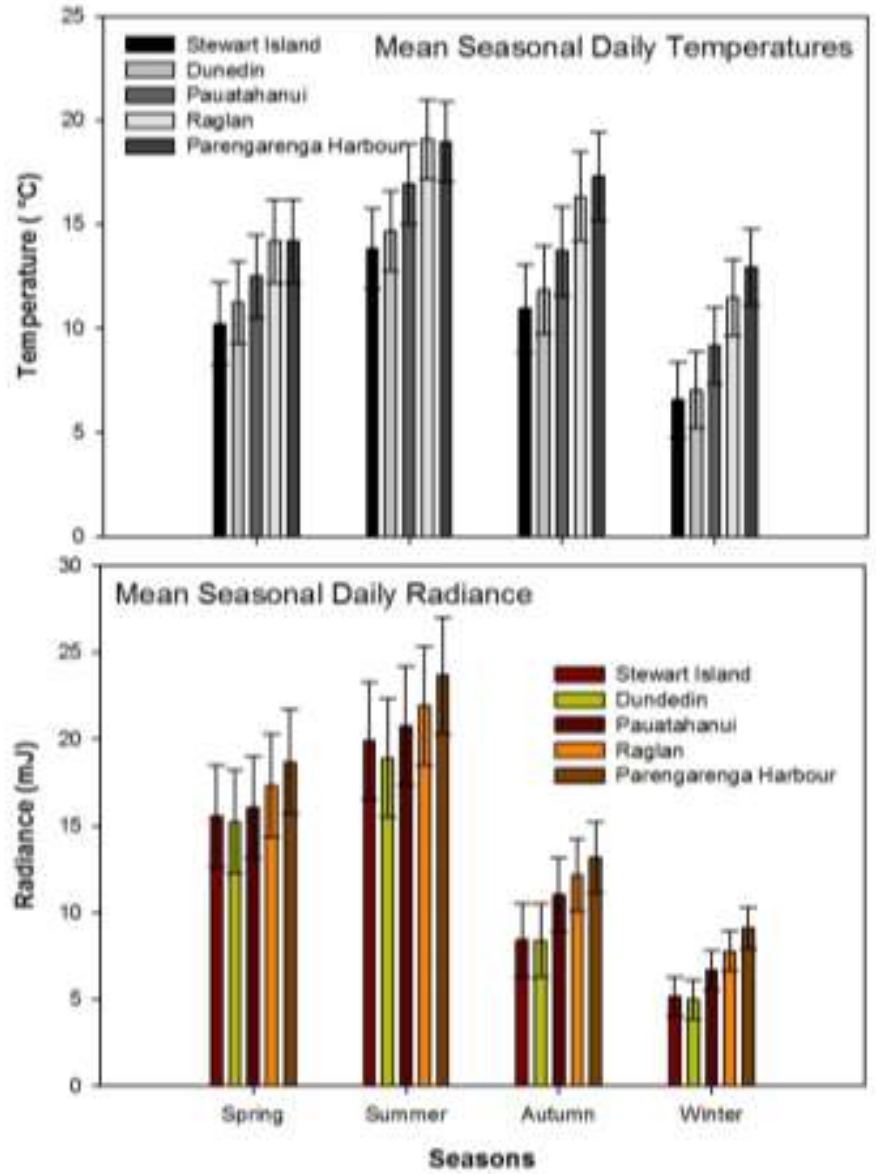

Figure A1.1.1: Mean ( \pm SE) daily temperature and radiance through the seasons for 5 years prior to the molecular latitudinal study (autumn 2007-summer 2012) for the 5 collection sites. Data for the 5 collection sites came from NIWA's National Climate Database (Cliflo Retrieved 6 June 2012). Tiwai Point station, Bluff for Stewart Island; Mussleburch Ews for Dunedin; Paraparaumu Aero Aws for Pauatahanui; Port Taharoa (temperature) and Hamiton Ruakura 2 Ews (radiation) for Raglan and; Cape Reinga Aws for Parengarenga Harbour. 


\section{$\underline{\text { A1.2Temperature and radiance at the seasonal and habitat sites }}$}
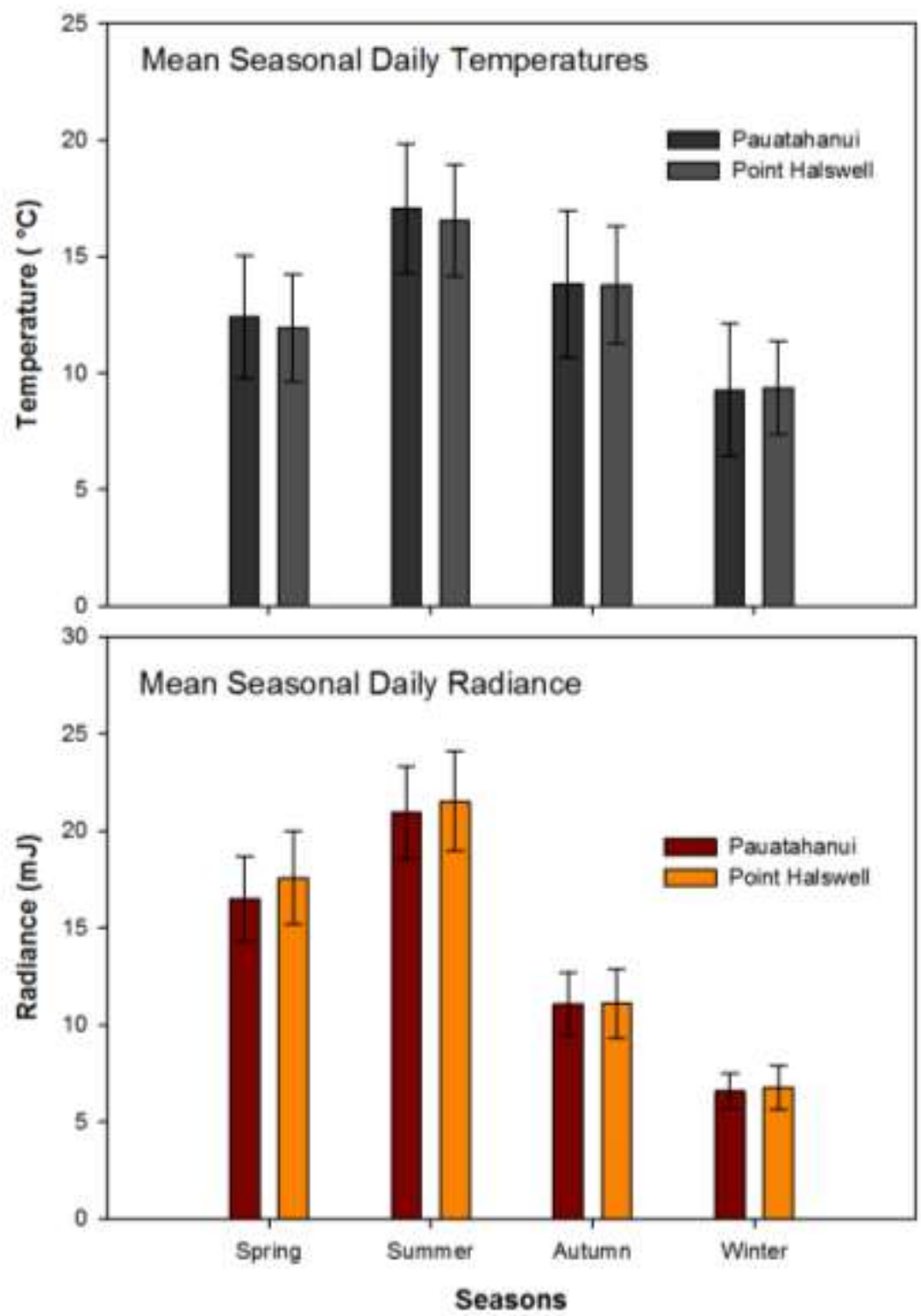

Figure A1.2.1: Mean $( \pm \mathrm{SE})$ daily temperature and radiance through the seasons for 5 years prior to the molecular seasonal and habitat study (autumn 2007-summer 2012) for the 2 collection sites. Data for the collection sites came from NIWA's National Climate Database (Cliflo Retrieved 6 June 2012). Paraparaumu Aero Aws for Pauatahanui and; Wellington Kelburn for Point Halswell. 


\section{$\underline{\text { A1.3 Weather stations used to collect climate data }}$}

Table A1.3.1: Collection sites and their geographical coordinates for the molecular and photophysiology studies with the weather stations used to collect climatic data from NIWA's National Climate Database (Cliflo Retrieved 6 June 2012)

\begin{tabular}{|c|c|c|c|}
\hline Collection Site & Coordinates & Weather Station & $\begin{array}{c}\text { Database } \\
\text { Station Agent } \\
\text { Number }\end{array}$ \\
\hline Stewart Island & $46^{\circ} 54^{\prime} 346^{\prime \prime} \mathrm{S}, 168^{\circ} 00^{\prime} 589^{\prime \prime} \mathrm{E}$ & Tiwai Point Ews & 5823 \\
\hline Dunedin & $45^{\circ} 46^{\prime} 60^{\prime \prime} \mathrm{S}, 170^{\circ} 41^{\prime} 60^{\prime \prime} \mathrm{E}$ & Mussleburch Ews & 15752 \\
\hline Pauatahanui & $41^{\circ} 6^{\prime} 4.85^{\prime \prime} \mathrm{S}, 174^{\circ} 52^{\prime} 17^{\prime \prime} \mathrm{E}$ & Paraparaumu Aero Aws & 8567 \\
\hline $\begin{array}{l}\text { Point Halswell, } \\
\text { Kau Bay }\end{array}$ & $41^{\circ} 17^{\prime} 2.42^{\prime \prime} \mathrm{S}, 174^{\circ} 49^{\prime} 33.89^{\prime \prime} \mathrm{E}$ & Wellington Kelburn & 25354 \\
\hline Raglan & $37^{\circ} 47^{\prime} 52^{\prime} ' \mathrm{~S}, 174^{\circ} 42^{\prime} 36^{\prime \prime} \mathrm{E}$ & $\begin{array}{l}\text { Port Taharoa (Temp) } \\
\text { Hamilton Ruakura (Rad) }\end{array}$ & $\begin{array}{c}2136 \\
12616\end{array}$ \\
\hline $\begin{array}{l}\text { Parengarenga } \\
\text { Harbour }\end{array}$ & $34^{\circ} 32^{\prime} 5^{\prime} \mathrm{S}, 172^{\circ} 56^{\prime} 43^{\prime \prime} \mathrm{E}$ & $\begin{array}{l}\text { Cape Reinga Aws (Temp) } \\
\text { Kaitaia Ews (Rad) }\end{array}$ & $\begin{array}{c}1002 \\
17067\end{array}$ \\
\hline
\end{tabular}




\section{APPENDIX 2 - Molecular sequences of Symbiodinium types in this study}

\section{A2.1 Coding used to identify samples in this study}

Table A2.1.1: Coding used in this study to identify the species of anemone the Symbiodinium cells came from, the place and habitat they originated from and the season they were collected.

\begin{tabular}{|l|l|l|}
\hline $\begin{array}{l}\text { Sample } \\
\text { code }\end{array}$ & Anemone species & Origin or place, habitat and season collected \\
\hline $\mathrm{Ab}$ & Anthopleura ballii & Collector's aquarium in Plymouth, England \\
\hline $\mathrm{Av}$ & Anemonia viridis & Plymouth, England \\
\hline AM & Aiptasia mutabilis & Roscoff in Brittany, France \\
\hline C & Cereus pedunculatus & Plymouth, England \\
\hline D & Anthopleura aureoradiata & Dunedin, New Zealand \\
\hline KA & Anthopleura aureoradiata & Kau Bay, autumn, midlittoral zone \\
\hline KU & Anthopleura aureoradiata & Kau Bay, summer, midlittoral zone \\
\hline KW & Anthopleura aureoradiata & Kau Bay, winter, midlittoral zone \\
\hline PA & Anthopleura aureoradiata & Pauatahanui Inlet, autumn \\
\hline PH & Anthopleura aureoradiata & Parengarenga Harbour, New Zealand \\
\hline PU & Anthopleura aureoradiata & Pauatahanui Inlet, summer \\
\hline PW & Anthopleura aureoradiata & Pauatahanui Inlet, winter \\
\hline W & Anthopleura aureoradiata & Raglan, New Zealand \\
\hline
\end{tabular}




\section{$\underline{\text { A2.2 Sequences of sample used for phylogenetic analysis }}$}

Table A2.2.1: DNA sequence alignment used for phylogenetic analysis obtained from Geneious v 5.6.2 (Drummond et al. 2011). First column is the identification code for each sample analysed.

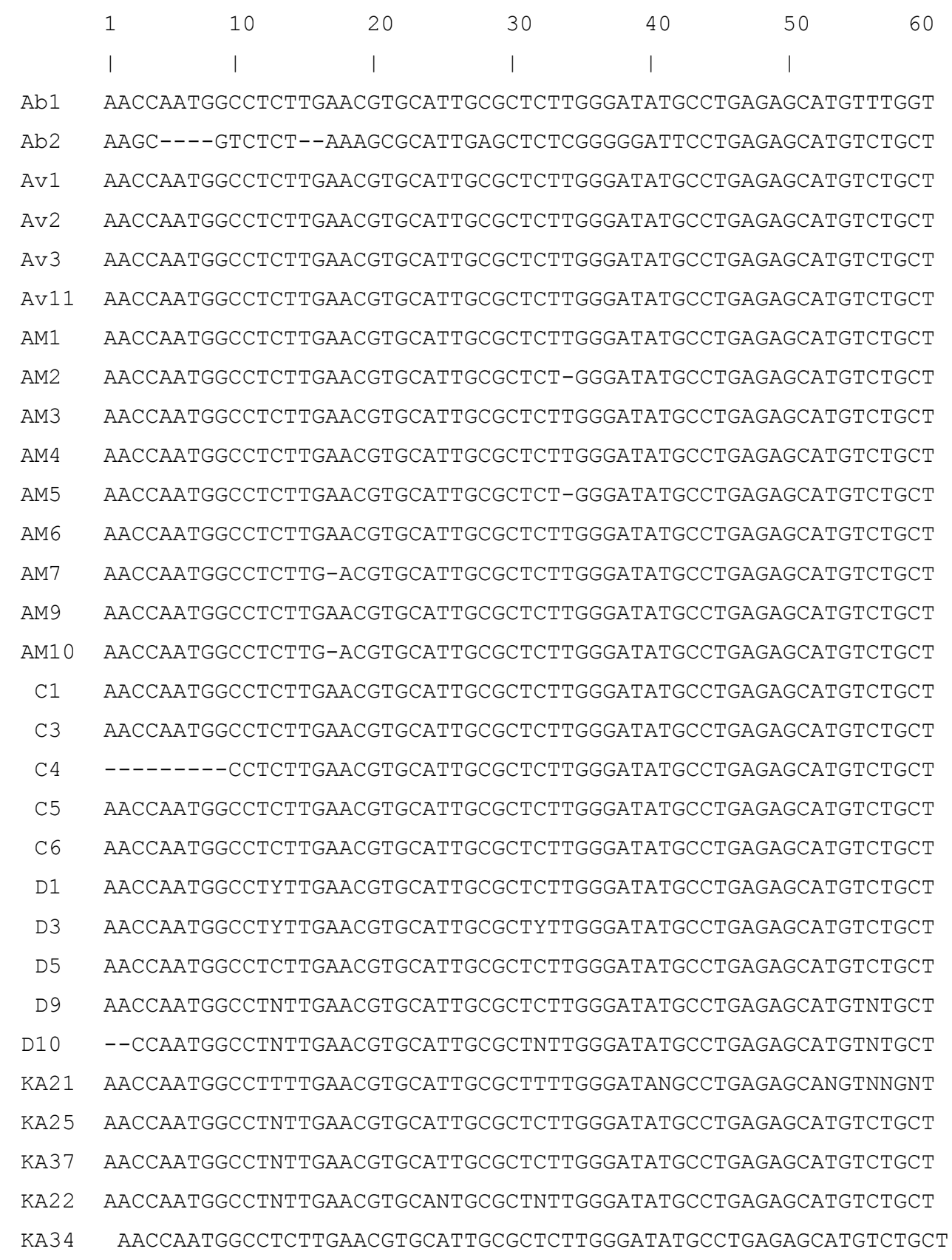




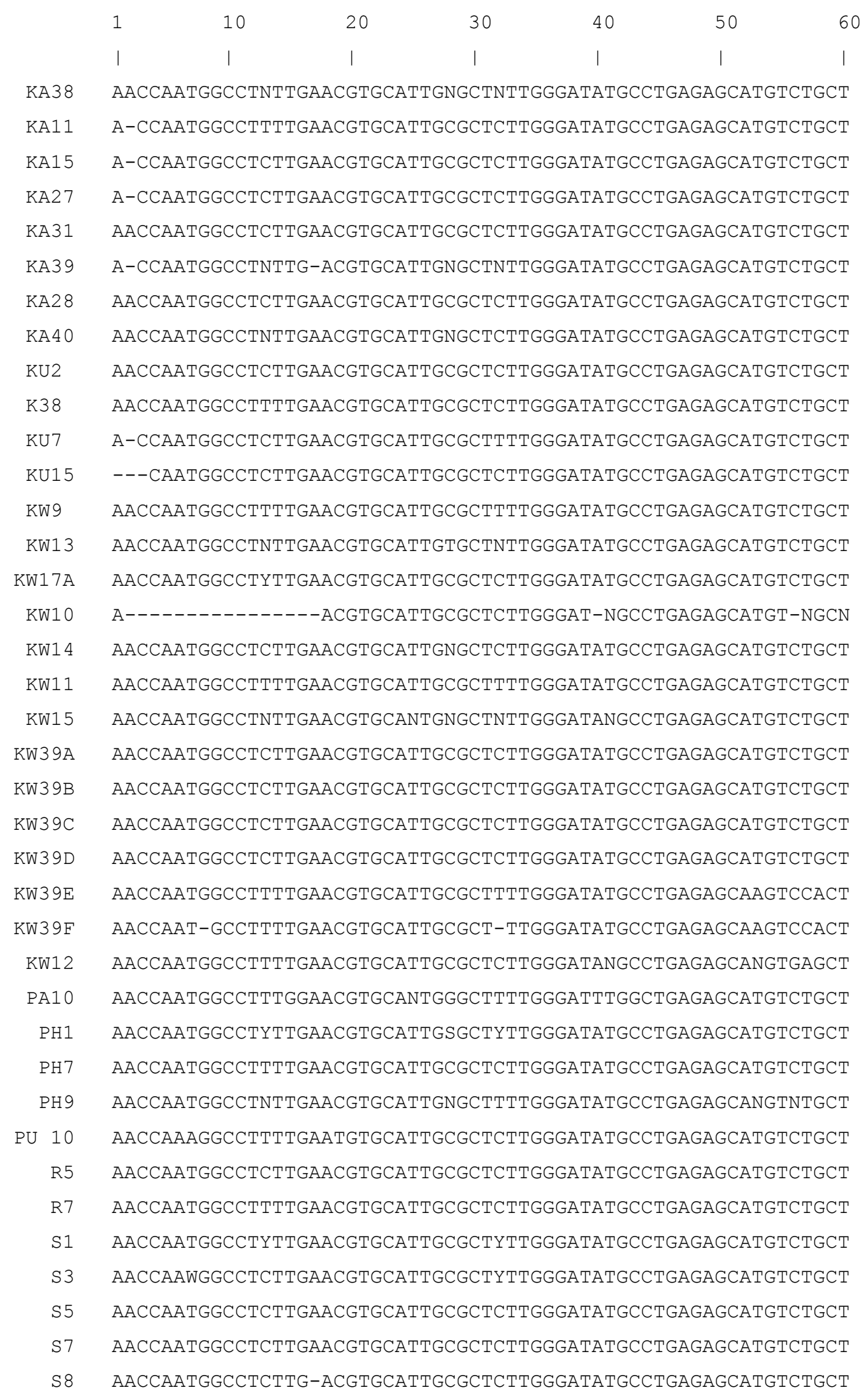




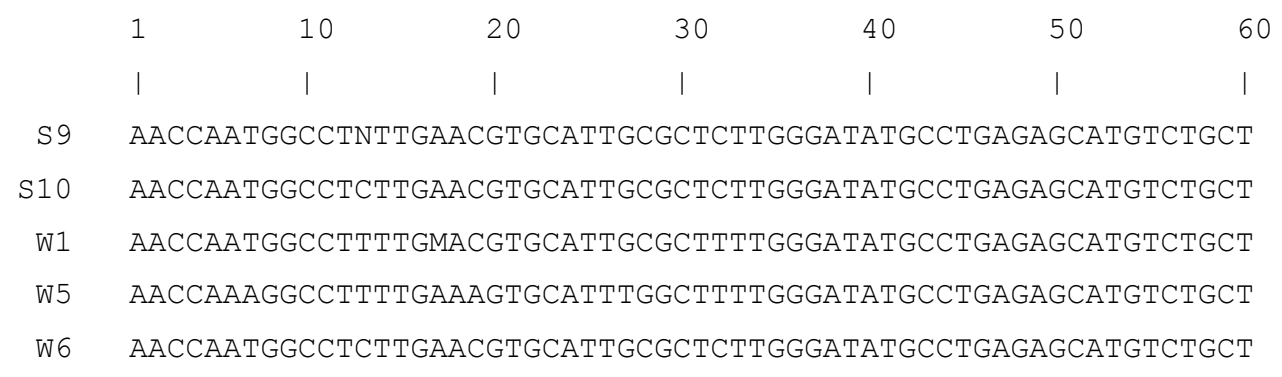

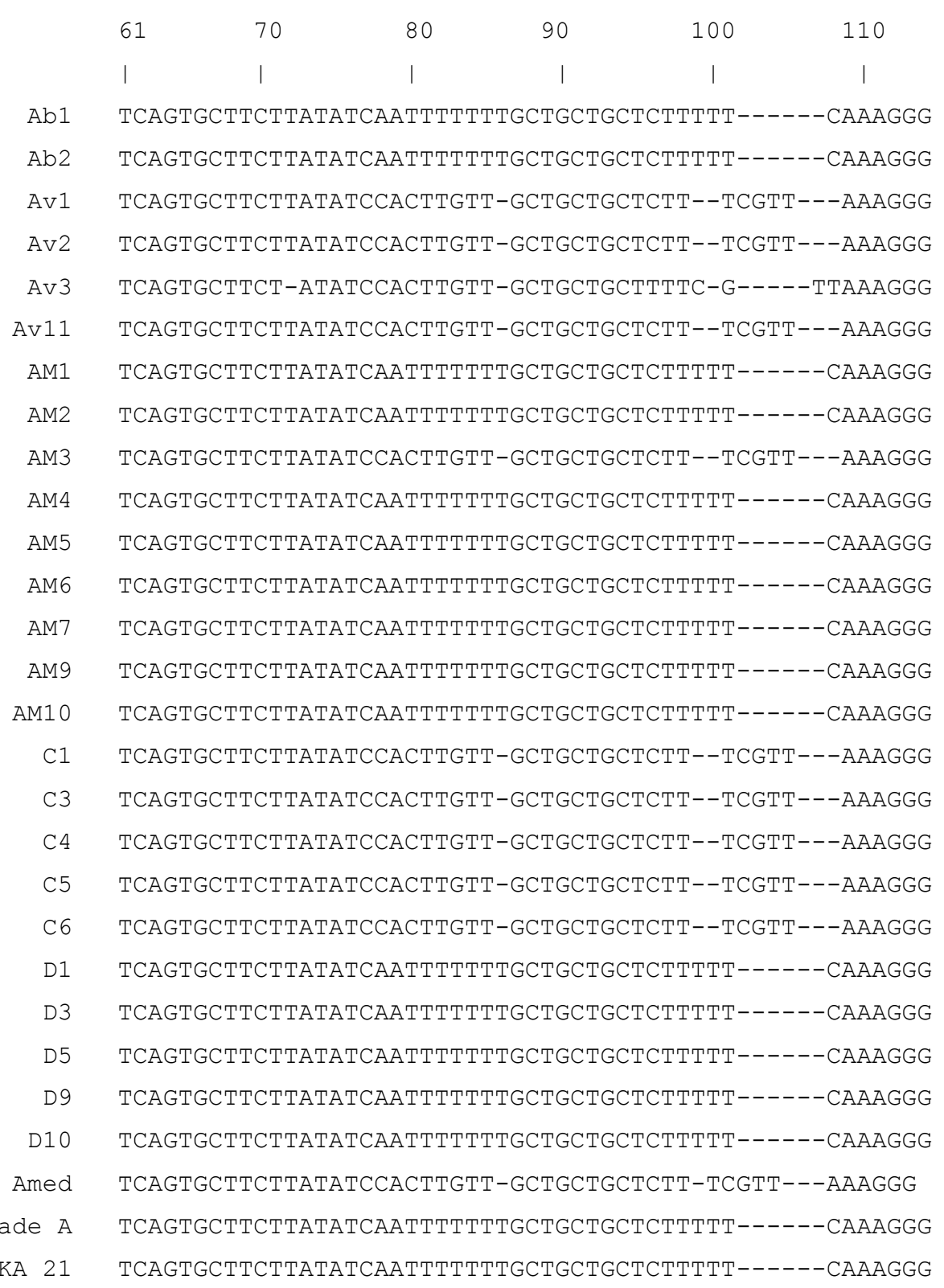




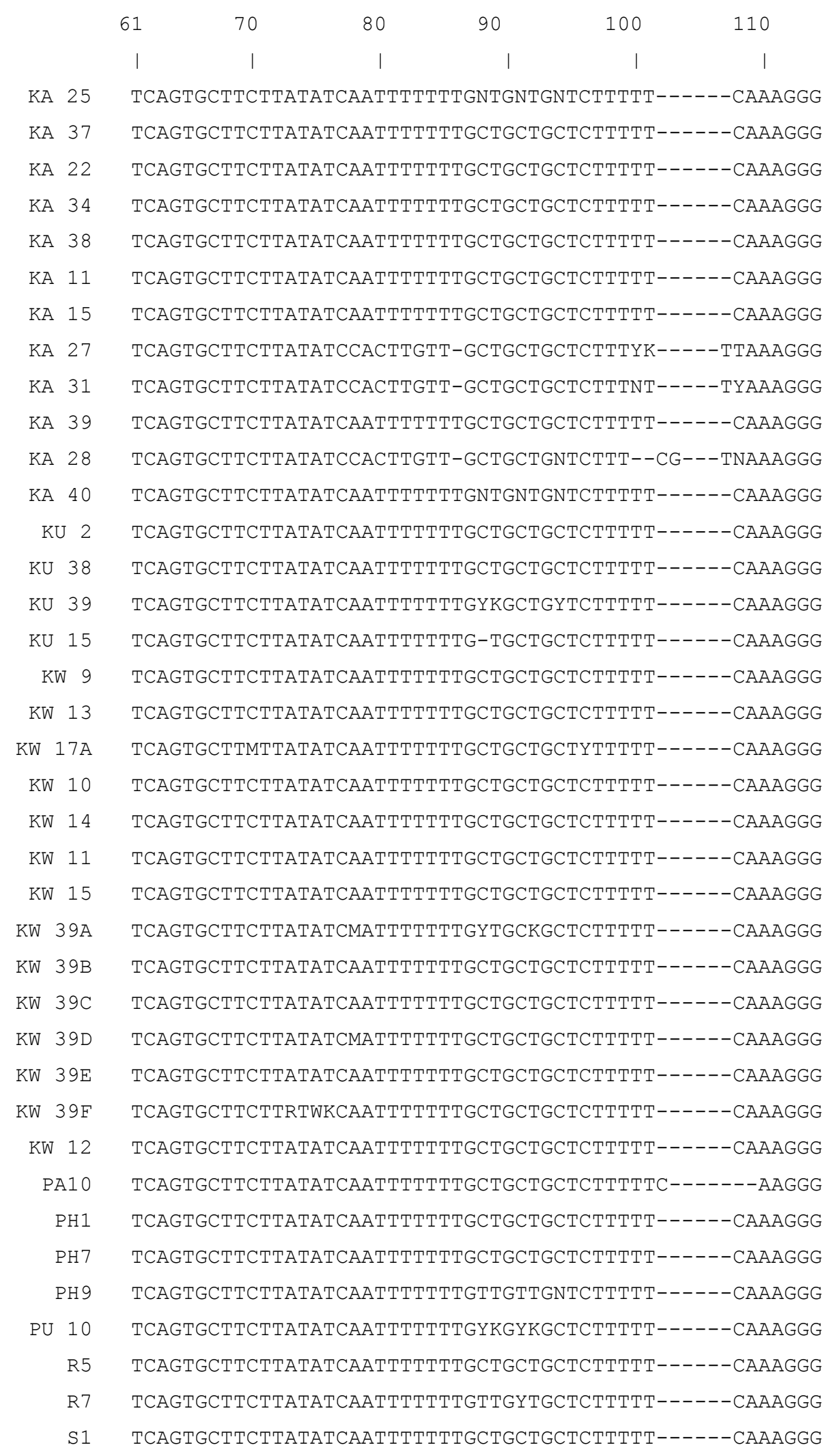




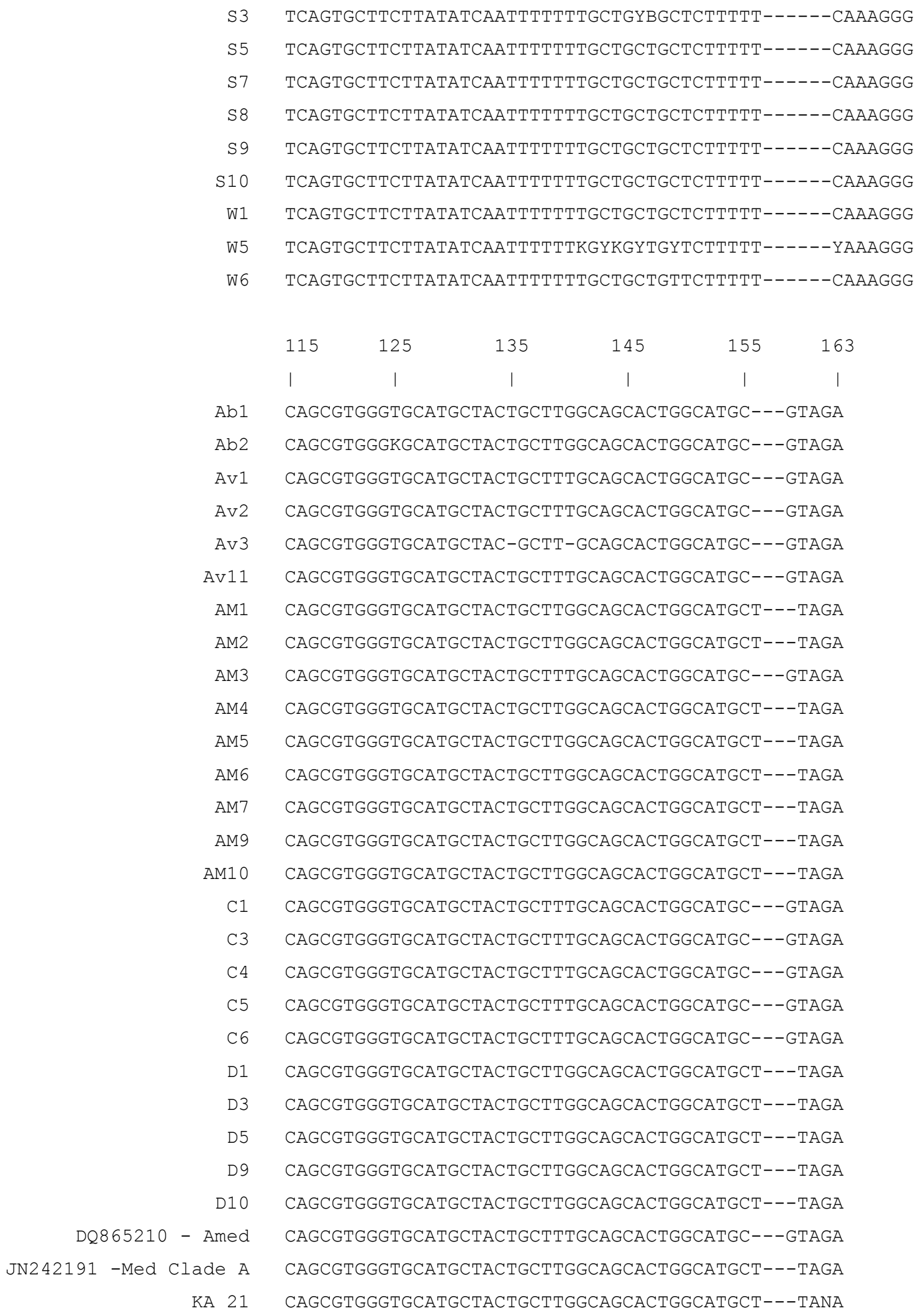




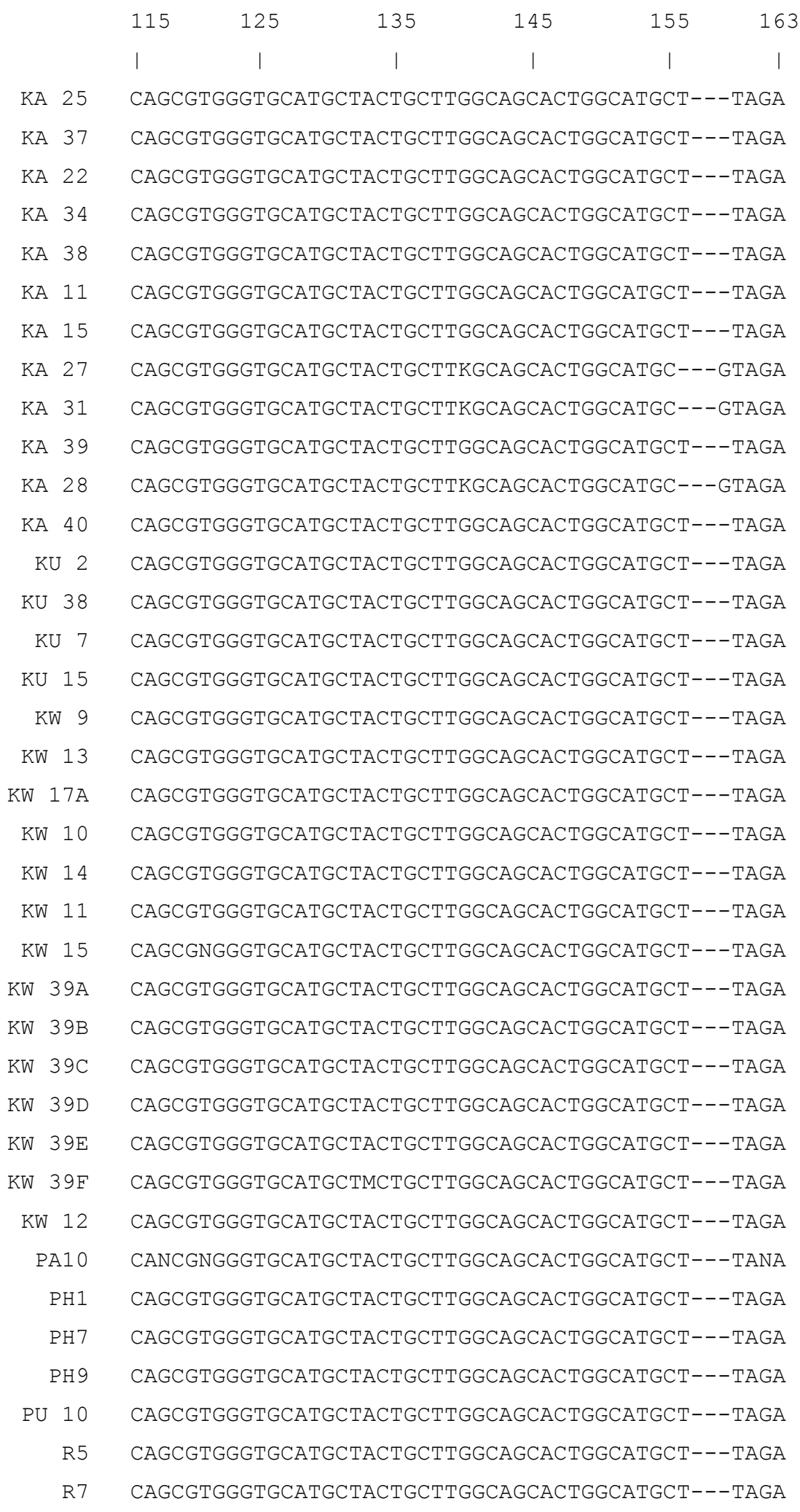




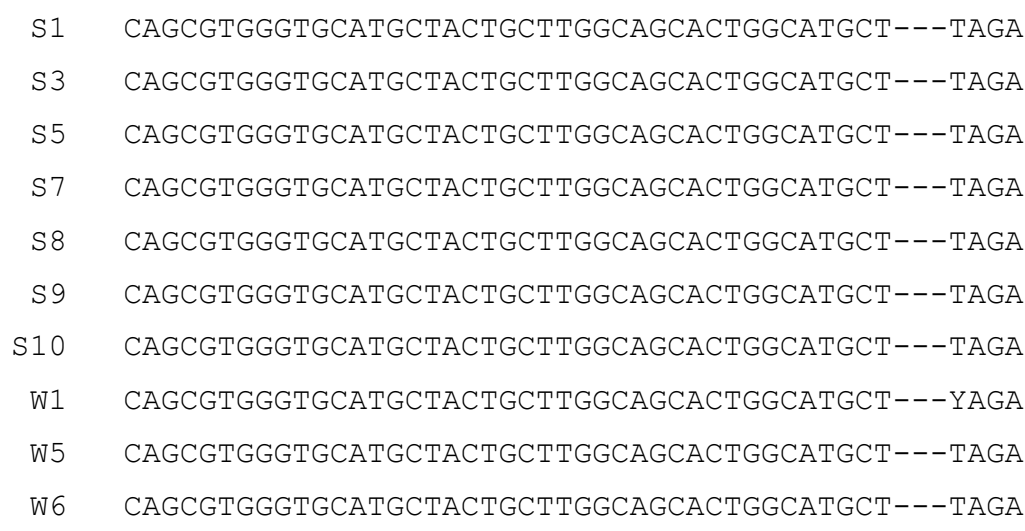

$\begin{array}{cccccc}164 & 174 & 184 & 194 & 204 & 214 \\ \text { | } & \mid & \mid & \mid & \mid & \text { | }\end{array}$

Ab1 TTTGCTGCTTGCTCA-CTGGTT-TGA-TTGATCACTKWATCAAAT-CTTGTC

Ab2 TTTGCTGCTTGCTCA-CTGGTT-TGA-TTGATCACTCTATCAAAT-CTTGTC

Av1 tTTGCTGCTtGCtCA-CTGgTtC-GA-TTGATCACTTCATCAAAT-CTTGTC

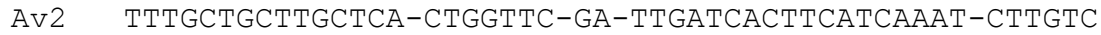

Av3 TTTGC-GCTTGCTCA-C-GGTTC-GA-TTGATCACTTCATCAAAT-CTTGTC

Av11 TTTGCTGCTTGCTCA-CTGGTTC-GA-TTGATCACTTCATCAAAT-CTTGTC

Am1 tTTGCTGCTTGCTCA-CTGGTT-TGA-TTGATCACTTTATCAAAT-CTTGTC

AM2 TTTGCTGCTTGCTCA-CTGGTT-TGA-TTGATCACTTTATCAAAT-CTTGTC

AM3 tTtGCTGCTtGCtCA-CTGGTtC-GA-TtGATCACTTCATCAAAT-CTTGTC

AM4 TTTGCTGCTTGCTCA-CTGGTT-TGA-TTGATCACTTTATCAAAT-CTTGTC

AM5 TtTGCTGCTtGCtCA-CTGGTT-TGA-TTGATCACTTtATCAAAT-CTTGTC

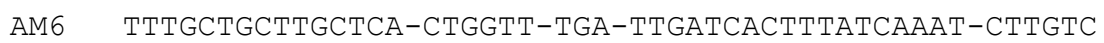

Am7 TTTGCTGCTTGCTCA-CTGGTT-TGA-TTGATCACTTTATCAAAT-CTTGTC

Am9 TTTGCTGCTTGCTCA-CTGGTT-TGA-TTGATCACTTTATCAAAT-CTTGTC

AM10 TTTGCTGCTTGCTCA-CTGGTT-TGA-TTGATCACTTTATCAAAT-CTTGTC

C1 TtTGCTGCTTGCTCA-CTGGTTC-GA-TTGATCACTTCATCAAAT-CTTGTC

C3 TTTGCTGCTTGCTCA-CTGGTTC-GA-TTGATCACTTCATCAAAT-CTTGTC

C4 TTTGCTGCTTGCTCA-CTGGTTC-GA-TTGATCACTTCATCAAAT-CTTGTC

C5 TTtGCTGCTTGCTCA-CTGGTTC-GA-tTGATCACTTCATCAAAT-CTTGTC

C6 TTTGCTGCTTGCTCA-CTGGTTC-GA-TTGATCACTTCATCAAAT-CTTGTC

D1 TTTGCTGCTTGCTCA-CTGGTT-TGA-TTGATCACTTTATCAAAT-CTTGTC

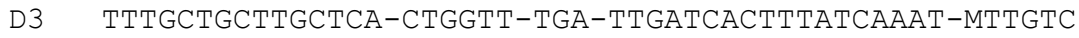

D5 TTTGCTGCTTGCTCA-CTGGTT-TGA-TTGATCACTTTATCAAAT-CTTGTC

D9 TTTGCTGCTTGCTCA-CTGGTT-TGA-TTGATCACTTTATCAAAT-NTTGTC

D10 TtTGCTGCTTGCTCA-CTGGTT-TGA-TTGATCACTTTATCAAAT-CTTGTC 
DQ865210 - Amed JN242191 -Med Clade A

KA 21

KA 25

KA 37

KA 22

KA 34

KA 38

KA 11

KA 15

KA 27

KA 31

KA 39

KA 28

KA 40

KU 2

KU 38

KU 7

KU 15

KW 9

KW 13

KW 17A

KW 10

KW 14

KW 11

KW 15

KW 39A

KW 39B

KW 39C

KW 39D

KW 39E

KW 39F

KW 12

PA10

$\mathrm{PH}$

$\mathrm{PH} 7$

PH 9

PU 10

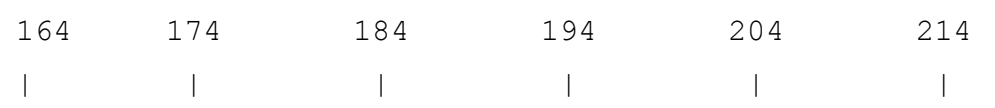

TTTGCTGCTTGCTCA-CTGGTTC-GA-TTGATCACTTCATCAAAT-CTTGTC TTTGCTGCTTGCTCA-CTGGTT-TGA-TTGATCACTTTATCAAAT-CTTGTC TTTGCTGCTTGCTCA-CTGGTT-TGA-TTGATCACTTTATCAAAT-NTTGTC TTTGCTGCTTGCTCA-CTGGTT-TGA-TTGATCACTTTATCAAATT-TTGTC TTTGCTGCTTGCTCA-CTGGTT-TGA-TTGATCACTTTATCAAAT-NTTGTC TTTGCTGCTTGCTCA-CTGGTT-TGA-TTGATCACTTTATCAAAT-NTTGTC TTTGCTGCTTGCTCA-CTGGTT-TGA-TTGATCACTTTATCAAAT-CTTGTC TTTGCTGCTTGCTCA-CTGGTT-TGA-TTGATCACTTTATCAAAT-CTTGTC TTTGCTGCTTGCTCA-CTGGTT-TGA-TTGATCACTTTATCAAAT-CTTGTC TTTGCTGCTTGCTCA-CTGGTT-TGA-TTGATCACTTTATCAAAT-MTTGTC TTTGCTGCTTGCTCA-CTGGTTC-GA-TTGATCACTTCATCAAAT-CTTGTC TTTGCTGCTTGCTCA-CTGGTTC-GA-TTGATCACTTCATCAAAT-CTTGTC TTTGCTGCTTGCTCA-CTGGTT-TGA-TTGATCACTTTATCAAAT-NTTGTC TTTGCTGCTTGCTCA-CTGGTTC-GA-TTGATCACTTCATCAAAT-CTTGTC TTTGCTGCTTGCTCA-CTGGTT-TGA-TTGATCACTTTATCAAAT-CTTGTC TTTGCTGCTTGCTCA-CTGGTT-TGA-TTGATCACTTTATCAAAT-CTTGTC TTTGCTGCTTGCTCA-CTGGTT-TGA-TTGATCACTTTATCAAAT-CTTGTC TTTGCTGCTTGCTCA-CTGGTT-TGA-TTGATCACTTTATCAAAT-CTTGTC TTTGCTGCTTGCTCA-CTGGTT-TGA-TTGATCACTTTATCAAAT-CTTGTC TTTGCTGCTTGCTCA-CTGGTT-TGA-TTGATCACTTTATCAAAT-MTTGTC TTTGCTGCTTGCTCA-CTGGTT-TGA-TTGATCACTTTATCAAAT-CTTGTC TTTGCTGCTTGCTCANCTGGTT-TGA-TTGATCACTTTWTCAAAT-MTTGTC TTTGCTGCTTGCTCA-CTGGTT-TGA-TTGATCACTTTATCAAAT-CTTGTC TTTGCTGCTTGCTCA-CTGGTT-TGA-TTGATCACTTTATCAAAT-NTTGTC TTTGCTGCTTGCTCA-CTGGTT-TGA-TTGATCACTTTATCAAAT-CTTGTC TTTGCTGCTTGCTCA-CTGGTT-TGA-TTGATCACTTTATCAAAT-CTTGTC TTTGCTGCTTGCTCA-CTGGTT-TGA-TTGATCACTTTATCAAAT-CTTGTC TTTGCTGCTTGCTCA-CTGGTT-TGA-TTGATCACTTTATCAAAT-CTTGTC TTTGCTGCTTGCTCA-CTGGTT-TGA-TTGATCACTTTATCAAAT-CTTGTC TTTGCTGCTTGCTCA-CTGGTT-TGA-TTGATCACTTTATCAAAT-CTTGTC TTTGCTGCTTGCTCA-CTGGTT-TGA-TTGATCACTTTATCAAAT-CTTGTC TTTGCTGCTTGCTCA-CTGGTT-TGA-TTGATCACTTTATCAAAT-CTTGTC TTTGCTGCTTGCTCA-CTGGTT-TGA-TTGATCACTTTATCAAAT-CTTGTC TTTGCTGCTTGCTCA-CTGGTT-TGA-TTGATCACTTTATCAAAT-CTTGTC TTTGCTGCTTGCTCA-CTGGTT-TGA-TTGATCACTTTATCAAATA-TTGTC TTTGCTGCTTGCTCA-CTGGTT-TGA-TTGATCACTTTATCAAATA-TTGTC TTTGCTGCTTGCTCA-CTGGTT-NGA-TTGATCACTTTATCAAAT-CTTGTC TTTGCTGCTTGCTCA-CTGGTT-TGA-TTGATCACTTTATCAAAT-CTTGTC 


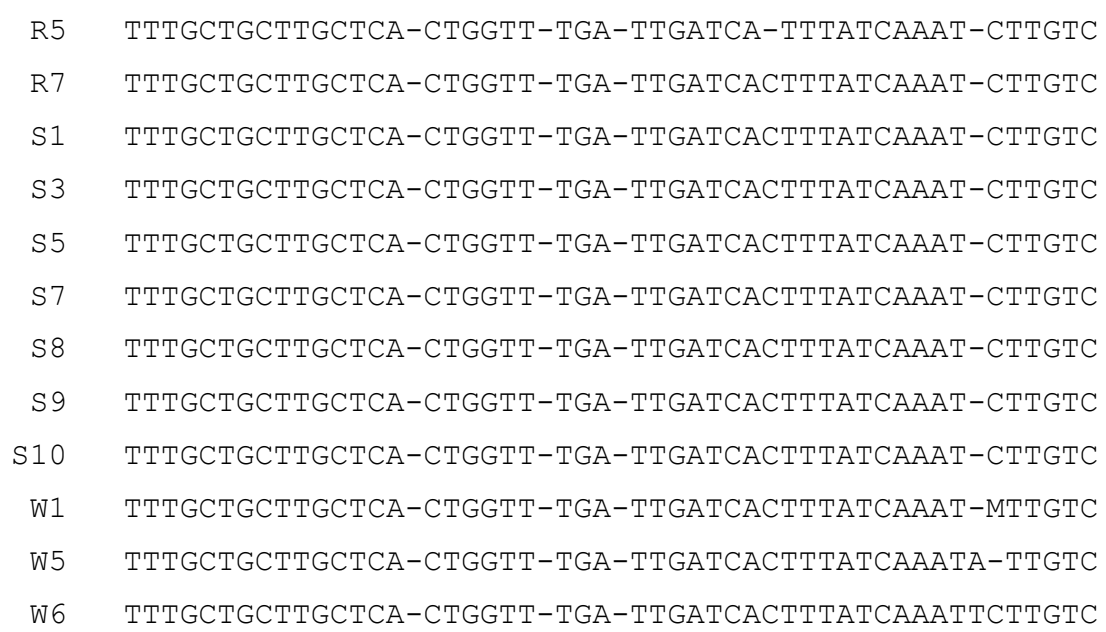

\begin{tabular}{ccccccc}
217 & 227 & 237 & 247 & 257 & 267 & 276 \\
\hline & $\mid$ & $\mid$ & $\mid$ & $\mid$ & $\mid$
\end{tabular}

Ab1 AgtATtGCA-AgtCTTGCGTAT-GTGTTGT-GACACATCCTAGCATGAAGTCAGACA-A

Ab2 AgTATTGCA-AgTCTTGCGTAT-GTGTTGT-GACACATCCTAGAAAGAA--CACACACA

Av1 AGTGTTGCA-AGTCTTGCGTAT-GTGTT-TTGACACATCCTAGCATGAAGTCAGACA-A

Av2 AgTGTTGCA-AgTCTTGCGTA-TGTGTT-TTGACACATCCTAGCATGAAGTCAGACA-A

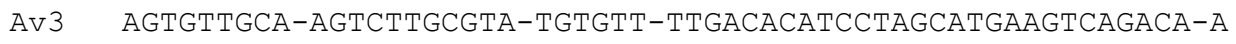

Av11 AgTGTTGCA-AGTCTTGCGTA-TGTGTT-TTGACACATCCTAGCATGAAGTCAGACA-A

AM1 AGTATTGCA-AGTCTTGTGTA-TGTGTTGT-GACACATCCTAGCATGAAGTCAGACA-A

Am2 AgtATtGCA-AGTCTTGTGTA-TGTGTTGT-GACACATCCTAGCATGAAGTCAGACA-A

AM3 AgTGTTGCA-AgtCTtGCGTA-TGTGTT-TTGACACATCCTAGCATGAAGTCAGACA-A

AM4 AgTATTGCA-AGTCTTGTGTA-TGTGTTGT-GACACATCCTAGCATGAAGTCAGACA-A

Am5 AgtATtGCA-AGTCTTGTGTA-TGTGTTGT-GACACATCCTAGCATGAAGTCAGACA-A

Am6 AgTATTGCA-AGTCTTGTGTA-TGTGTTGT-GACACATCCTAGCATGAAGTCAGACA-A

Am7 AgtATtGCA-AgtCTtGTGtA-TGtgtTGt-GACACATCCTAGCATGAAGTCAGACA-A

Am9 AgtATTGCA-AGTCTTGTGTA-TGTGTTGT-GACACATCCTAGCATGAAGTCAGACA-A

Am10 AgtATtGCA-AGTCTTGTGTA-TGTGTTGT-GACACATCCTAGCATGAAGTCAGACA-A

C1 AgtGTtGCA-AgtCTTGCGTA-TGtgtT-tTGACACATCCTAGCATGAAGTCAGACA-A

C3 AgtGTTGCA-AGTCTTGCGTA-TGTGTT-tTGACACATCCTAGCATGAAGTCAGACA-A

C4 AgTGTTGCA-AGTCTTGCGTA-TGTGTT-TTGACACATCCTAGCATGAAGTCAGACA-A

C5 AgtGTtGCA-AGTCTTGCGTA-TGTGtT-TTGACACATCCTAGCATGAAGTCAGACA-A

C6 AgtGTtGCA-AGTCTTGCGTA-TGTGTT-TTGACACATCCTAGCATGAAGTCAGACA-A

D1 AgtATtGCA-AGTCtTGtGtA-tgtgtTGt-GACACATCCTAGCATGAAGTCAGACA-A 


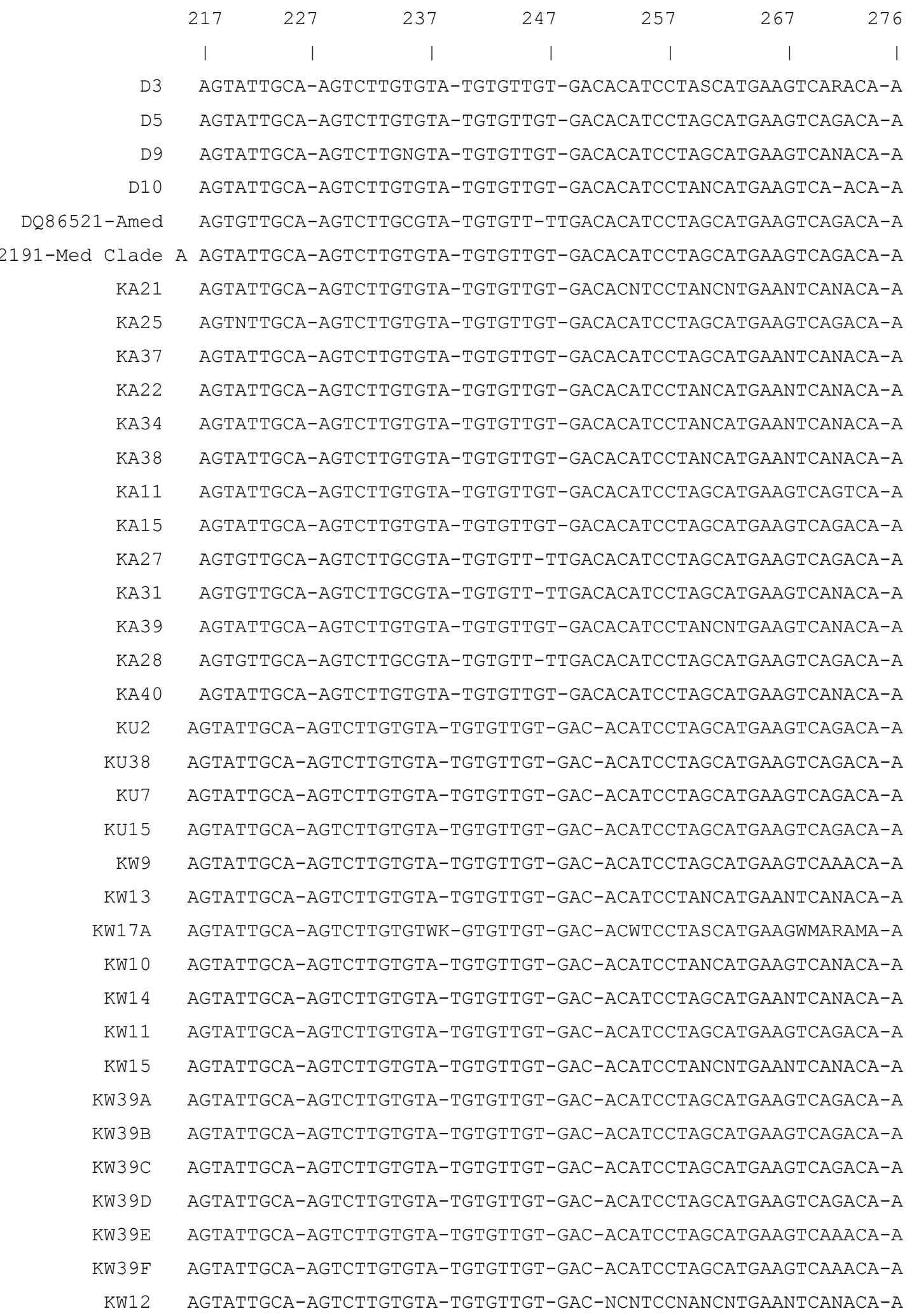




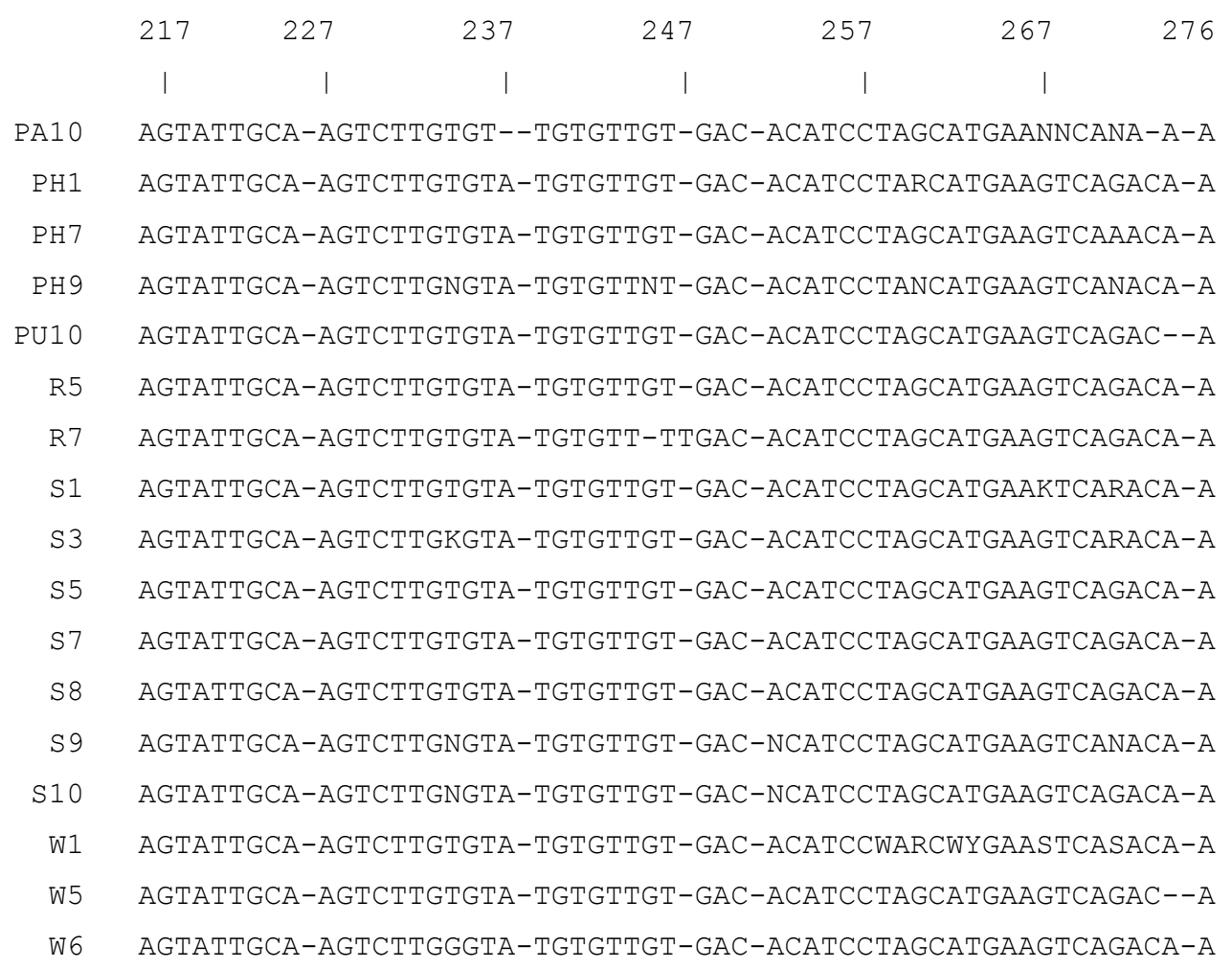




\section{$\underline{\text { A2.3 Sequences of Reference Symbiodinium Types }}$}

Table A2.3.2: DNA sequence alignment of reference Symbiodinium types used for phylogenetic analysis obtained from Geneious v 5.6.2 (Drummond et al. 2011). Sequences retrieved from the National Centre for Biotechnology Information (NCBI) BLAST database and from the ITS2 sequence database (GeoSymbio) from the Gates Laboratory at HIMB (Franklin et al. 2012)

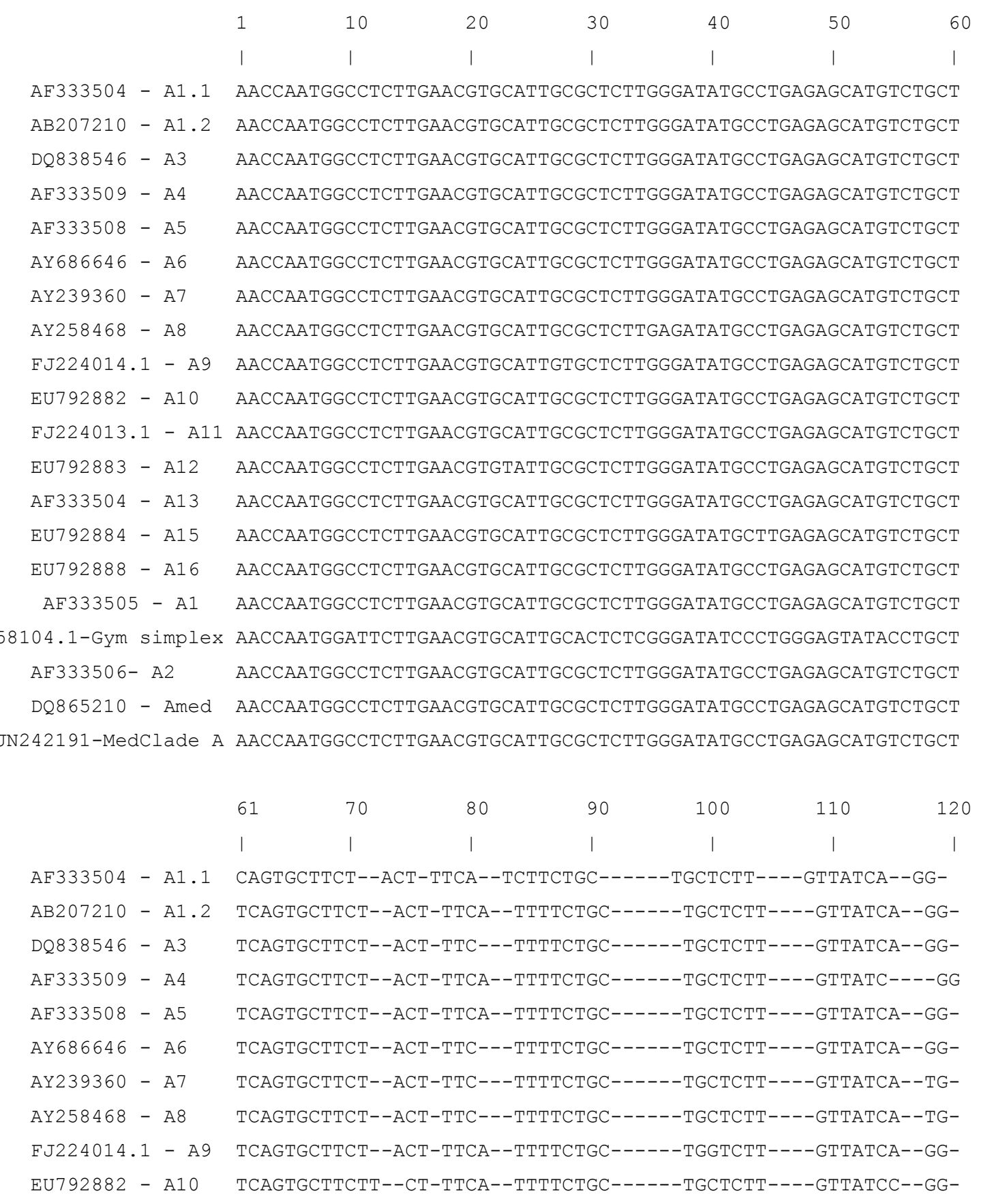




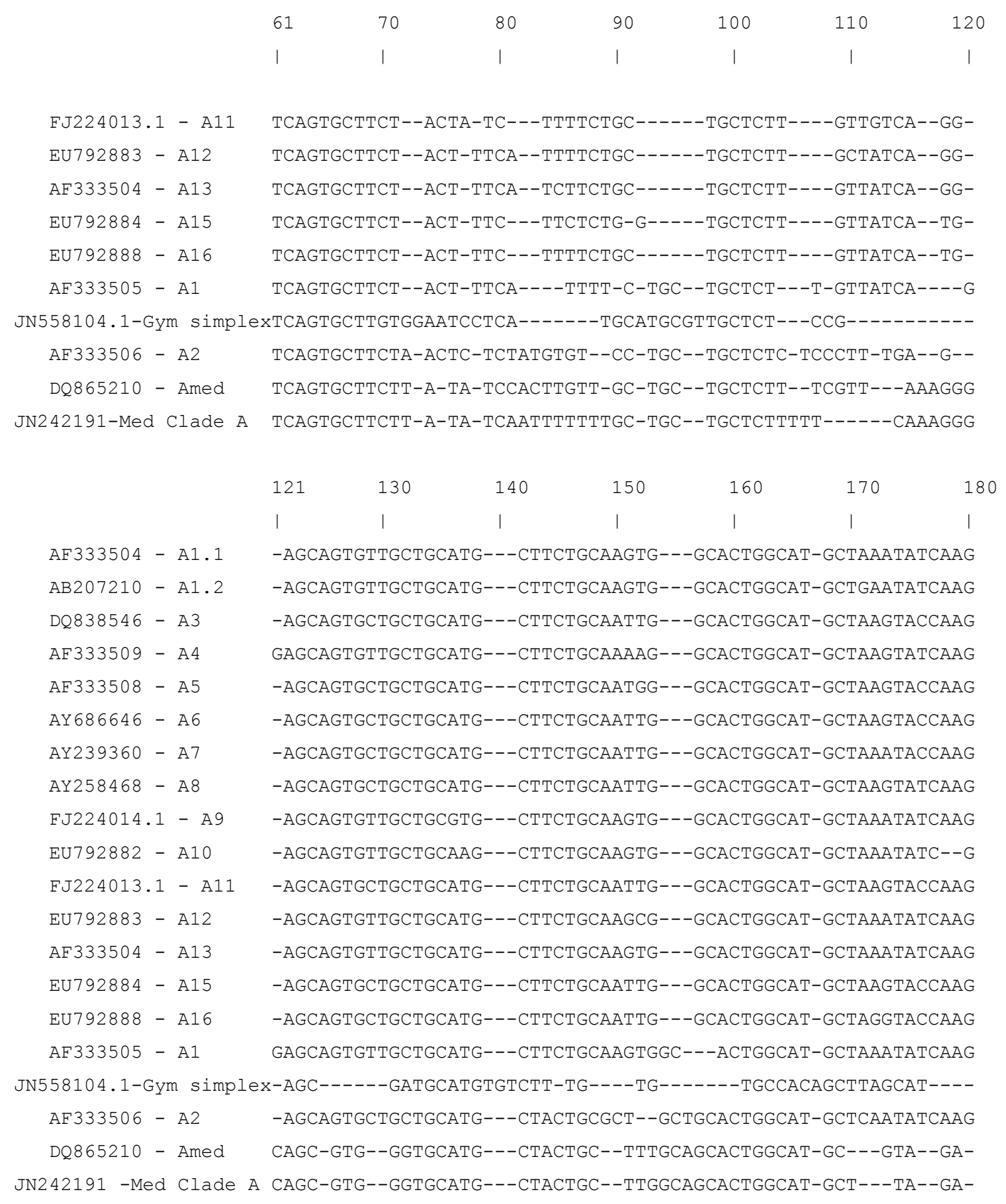




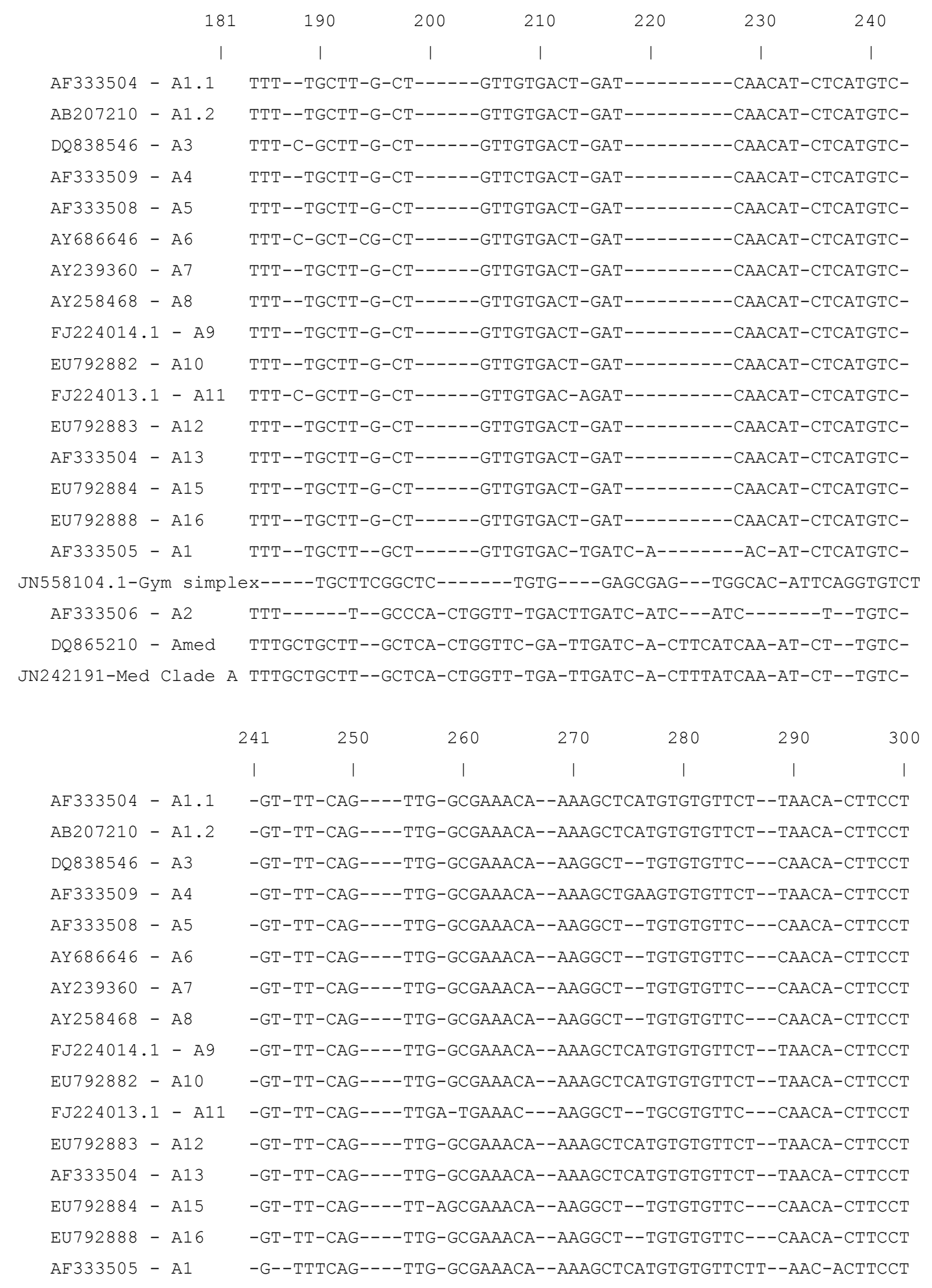

JN558104.1-Gym simplexAG-ATTGC-----CTTG-GCA---------------------TCT----ACAACTT--AF333506 - A2 ----TTGCAGA---TTGGGTAAAATAGGAAAT------AGTGTT--TGCAAC-ACTTCCT 


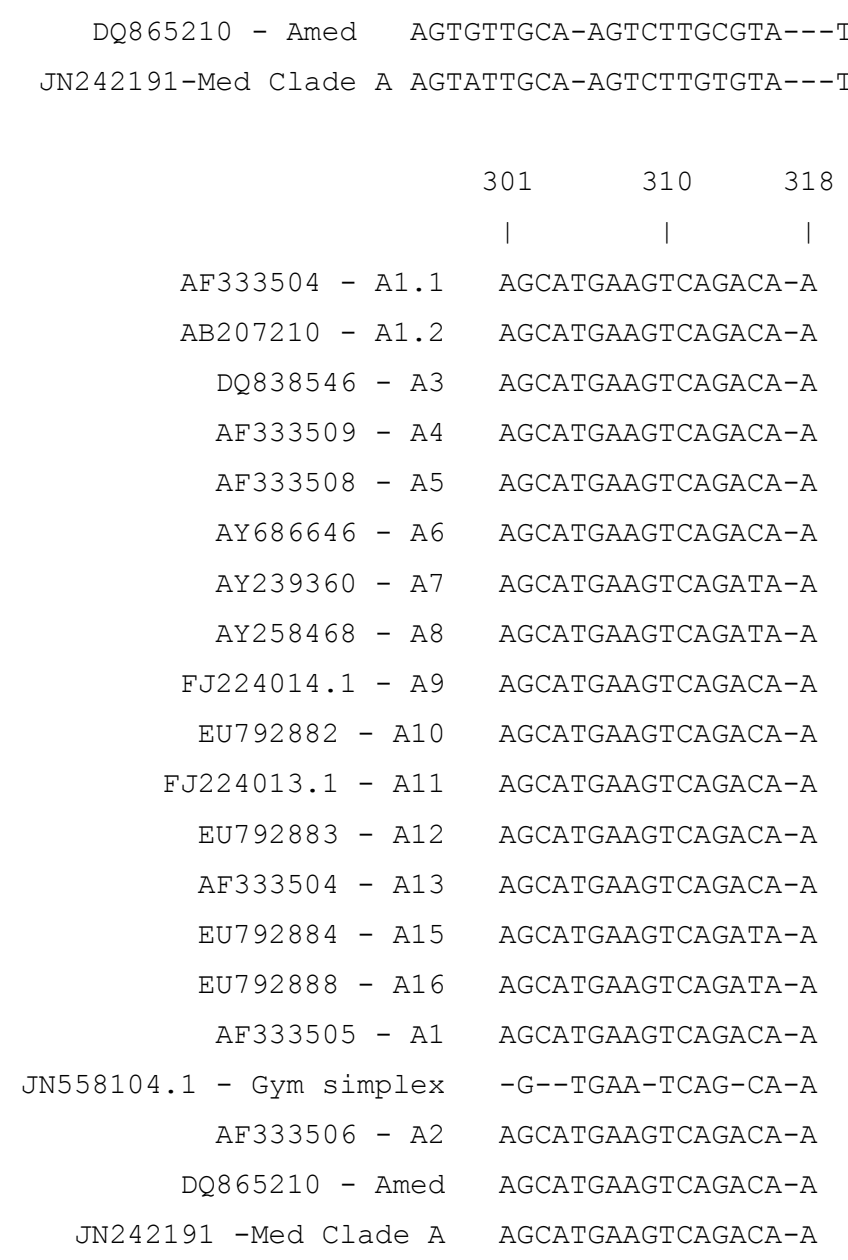




\section{A2.4 ITS2 secondary structures for Symbiodinium Clade A}

Figure A2.4.1: ITS2 secondary structures for Symbiodinium clade A as proposed by Hunter et al (2007) A) ITS2 type A1; B) ITS2 type “Amed"; C) ITS2 type A3; D) ITS2 type A4 (S. linucheae); E) ITS2 type "Cryptic A"; F) ITS2 type A2 (S. pilosum).

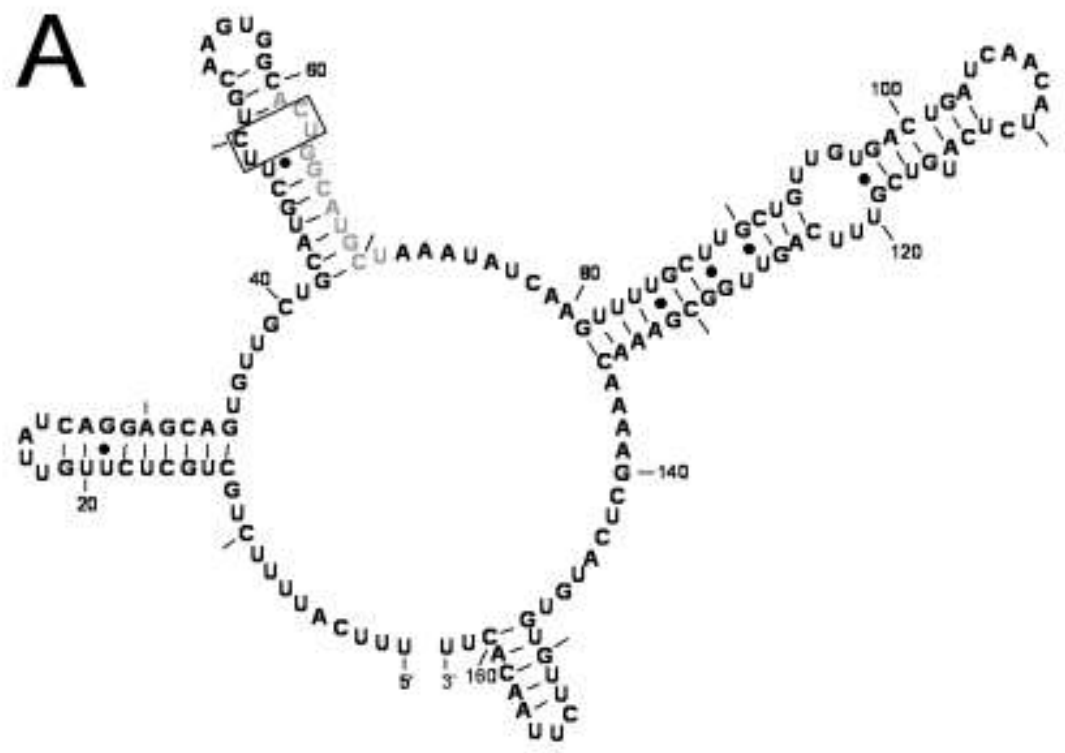

ITS2 type A1

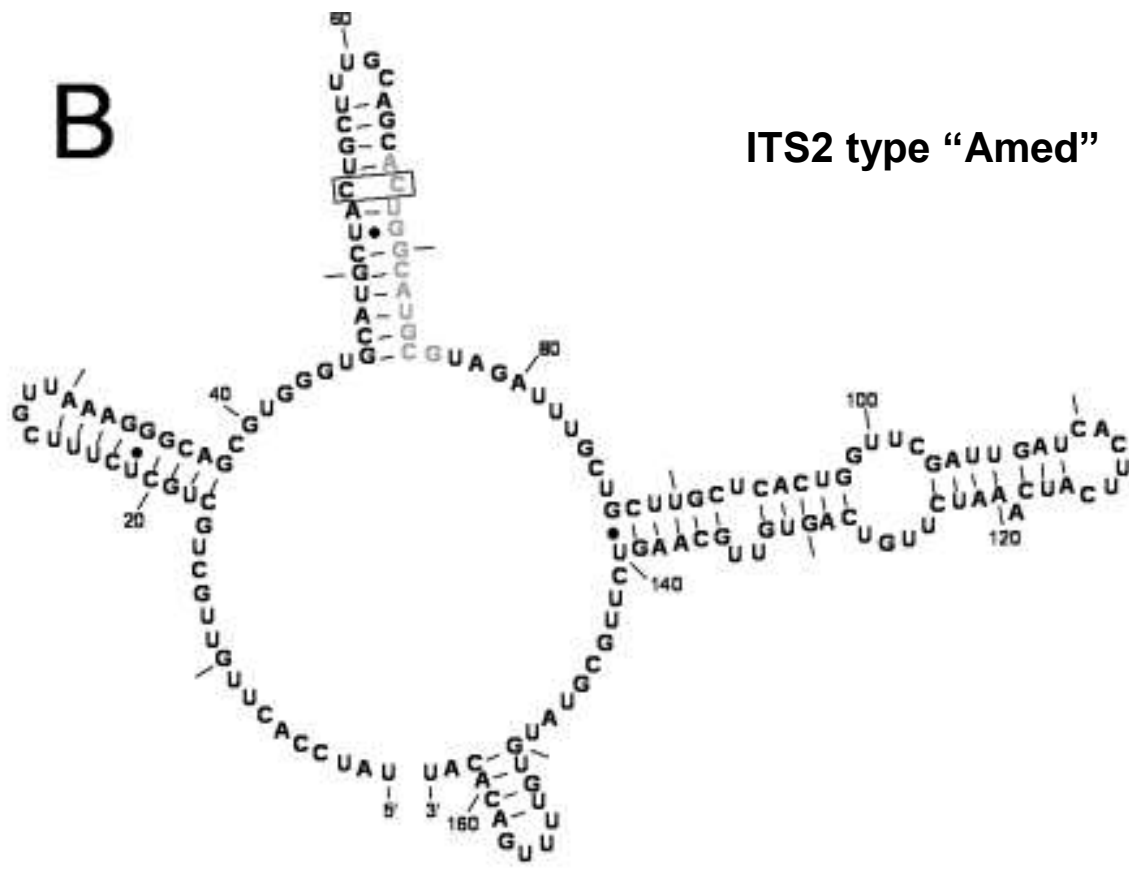



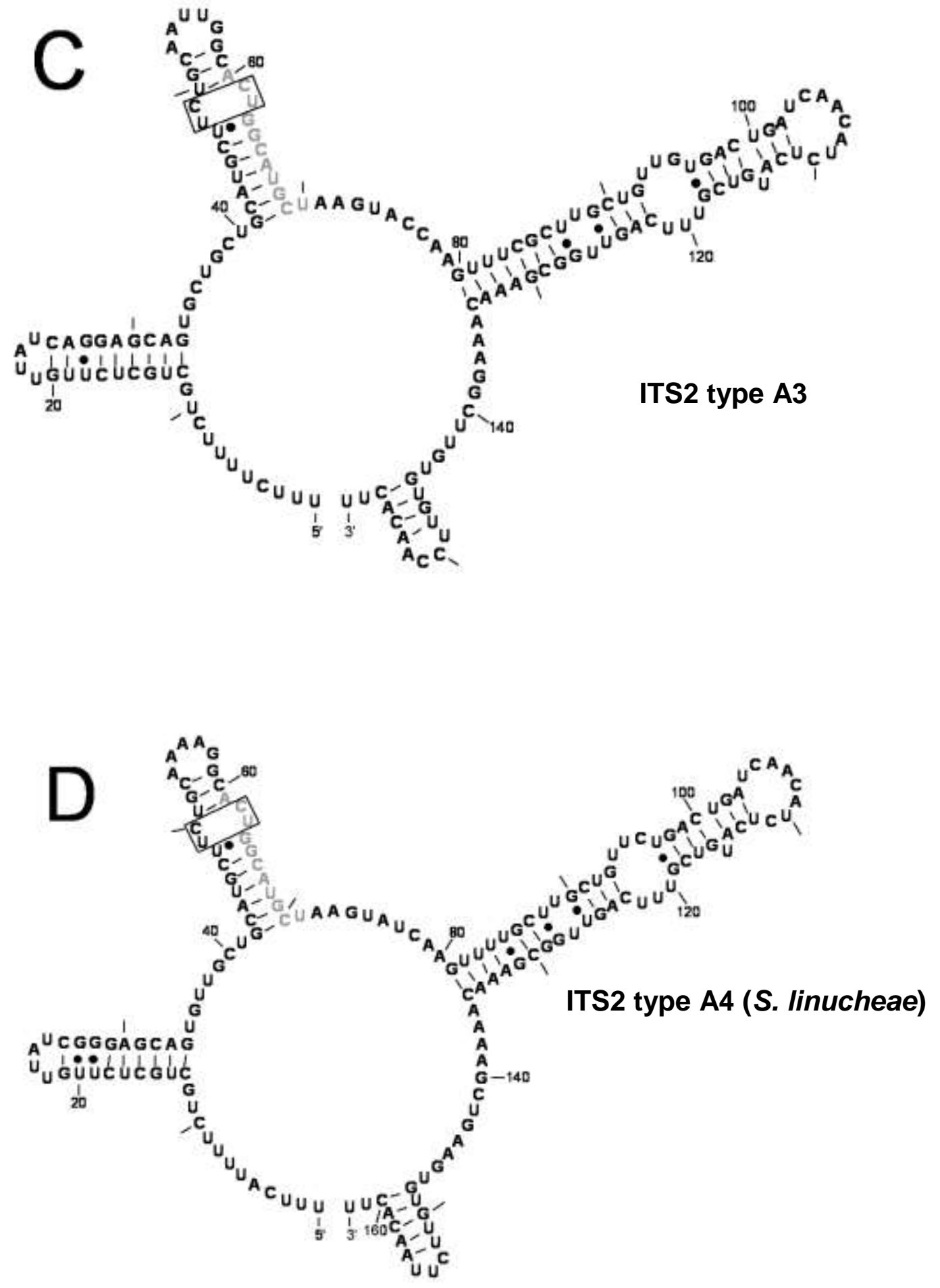

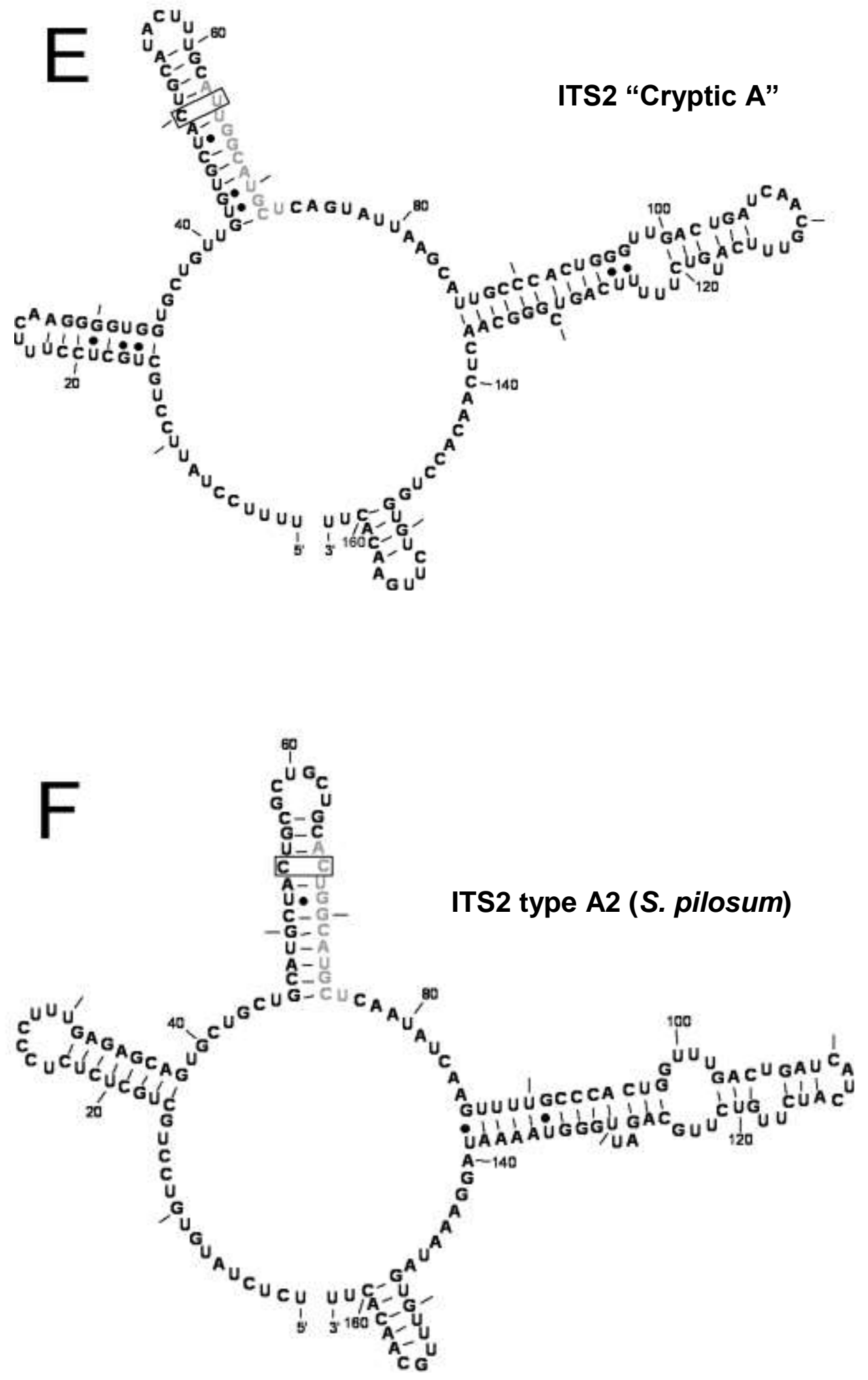


\section{APPENDIX 3- Preliminary trials on Anthopleura aureoradiata}

\section{$\underline{\text { A3.1 Introduction }}$}

The original proposal for my Masters' research was to carry out a set of experiments based on the study by Hill et al (2005). The ratios of the three components of NPQ in coral were measured after being placed in different treatments, with light and temperature as factors. The study found that the main component of NPQ was the energy dependent quenching (qE) which dissipates excess energy in non-bleaching stress conditions, either high light or high temperature. However, once bleaching conditions were enforced on the coral (high light and high temperature combined) total NPQ increased and the ratio of the different NPQ components changed. State transition quenching (qT) increased significantly compared to the other two components so that it became an equal contributor for energy dissipation as $\mathrm{qE}$. In all of the treatments, photoinhibitory quenching (qI) did not change in proportion to the whole.

The method used by Hill et al was to measure fluorescence emissions using a PAM fluorometer and analyse the relaxation kinetics of the chloroplasts in the Symbiodinium cells by adding DCMU (3-(3,4-dichlorophenyl)-1, 1-dimethylurea) after the cells had been dark adapted and once a series of saturating pulses had been applied. By using the data from the curve, the three components could be calculated. DCMU inhibits the formation of the $\Delta \mathrm{pH}$ across the thylakoid membrane by taking the place of the electron donor side on $Q_{B}$ (Jones and Kerswell 2003, Hill et al. 2005). Once DCMU binds the electron, it stops the onwards movement of the electron through the electron transport chain (Jones 2004). Therefore, once added, the relaxation of the $\Delta \mathrm{pH}$ can be measured and visualized on a graph and calculations made. 


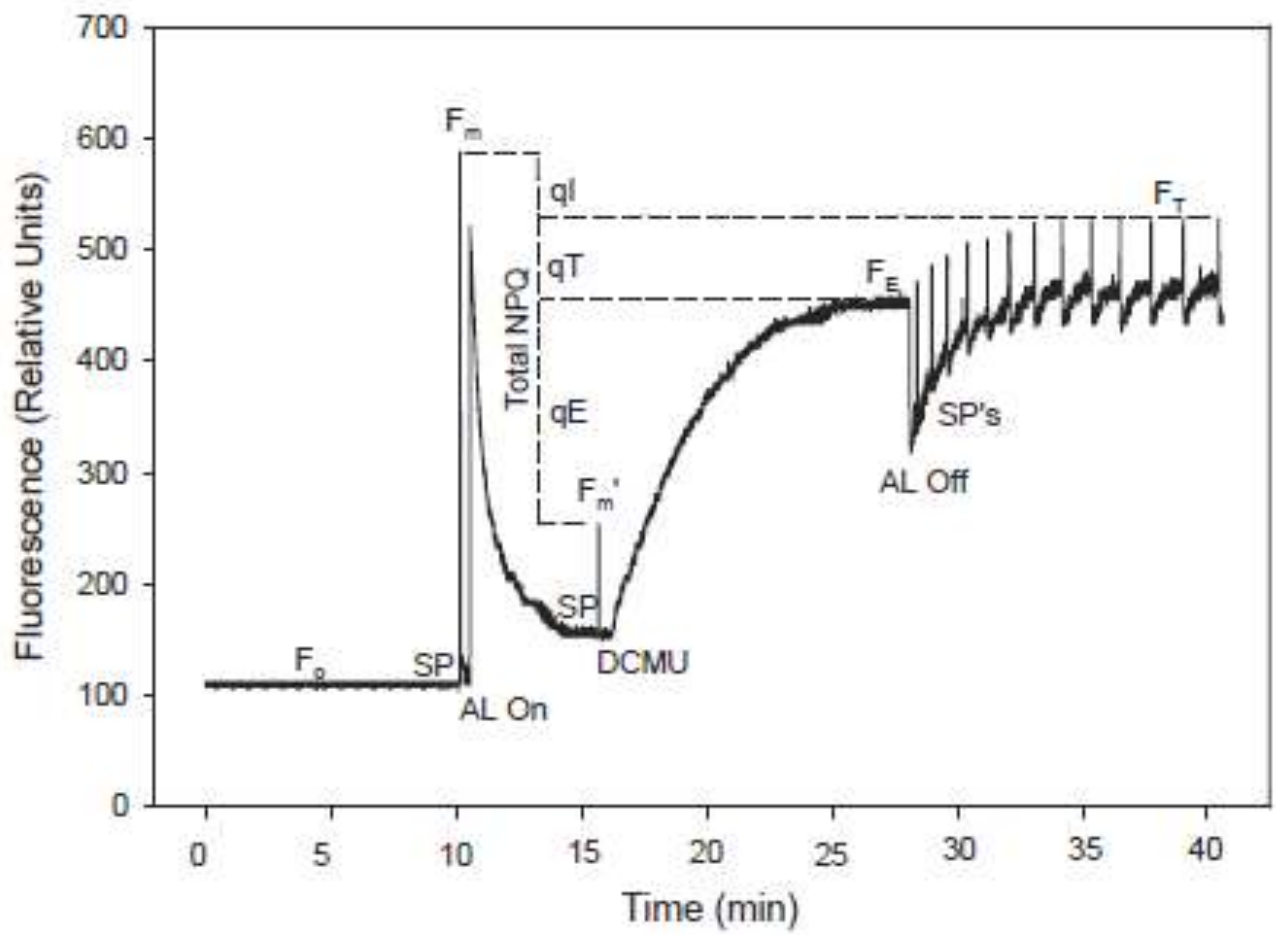

Figure A3.1.1: Chlorophyll fluorescence induction kinetics using DCMU to differentiate between the three components of NPQ. The kinetic curve depicts a control sample as produced in Hill et al's (2005) paper. SP saturating pulse, $\mathrm{AL}$ - actinic light.

From the diagram, total NPQ is measured as the distance between the first two saturation pulses $\left(\left(\mathrm{F}_{\mathrm{m}}-\mathrm{F}_{\mathrm{m}}{ }^{\prime}\right) / \mathrm{F}_{\mathrm{m}}{ }^{\prime}\right)$. $\mathrm{qE}$ occurs between the top of the second pulse and the plateauing of the fluorescence measurement once DCMU is added $\left(\mathrm{qE}=\left(\mathrm{F}_{\mathrm{E}}-\mathrm{F}_{\mathrm{m}}{ }^{\prime}\right) / \mathrm{F}_{\mathrm{m}}{ }^{\prime}\right)$. $\mathrm{qT}$ is measured from the plateauing at the end of $\mathrm{qE}$, when the actinic light is turned off, and the plateauing of the saturation pulses, which are applied every 30 seconds $\left(\mathrm{qT}=\left(\mathrm{F}_{\mathrm{T}}-\mathrm{F}_{\mathrm{E}}\right) / \mathrm{F}_{\mathrm{m}}{ }^{\prime}\right)$. $\mathrm{qI}$ is measured by the remaining difference between $\mathrm{F}_{\mathrm{T}}$ and $\mathrm{F}_{\mathrm{m}}\left(\left(\mathrm{F}_{\mathrm{m}}-\mathrm{F}_{\mathrm{T}}\right) / \mathrm{F}_{\mathrm{m}}{ }^{\prime}\right)$.

\section{$\underline{\text { A3.2 Trial Experiments }}$}

The first trial was to measure the concentration of DCMU needed to relax $\Delta \mathrm{pH}$ in the Symbiodinium cells in A. aureoradiata. I placed 1 anemone within each $3 \mathrm{ml}$ tube of filtered seawater (FSW) and placed 18 tubes within a holder in the PAM fluorometer. I added 6 different concentrations of DCMU to the tubes during the fluorescence run so that 
there were 3 tubes with the same concentration. The first problem encountered was that the anemones were very active during the PAM fluorometer run. They contracted their tentacles when put in the dark for the dark adaption stage and extended and withdrew their tentacles under the different light settings. This made any comparison between treatments impossible.

The next trial was to try to "relax" the anemones in an open state so that they could not move during the PAM fluorometer run. I did this by adding $\mathrm{MgCl}$ to the FSW. Firstly the anemones were given time to attach themselves to the bottom of the tubes within $100 \mu \mathrm{l}$ of FSW under a light source. After 15 minutes another $900 \mu \mathrm{l}$ of FSW was slowly added. After another 15 minutes another $1000 \mu \mathrm{l}$ of FSW was added. Once the anemones were all open, $\mathrm{MgCl}$ dissolved in FSW was added.

Different concentrations of $\mathrm{MgCl}$ were trialled (0.05 M, 0.1 M and 0.2 M). The two more concentrated solutions were too strong as the anemones curled up or became so flaccid that they no longer were able to retain attachment to the tubes. I also found that the addition of the $0.05 \mathrm{M} \mathrm{MgCl}$ solution caused some anemones to retract their tentacles due to the movement of water around them. Therefore, instead of adding $\mathrm{MgCl}$ solution to each $3 \mathrm{ml}$ tube, I attached the anemones as before but then placed the tubes within a bath of FSW under a light source. The bath water was maintained at $20{ }^{\circ} \mathrm{C}$. After 2 hours, when the anemones were open, $\mathrm{MgCl}$ solution was added to the bath so that the final concentration within the bath was $0.05 \mathrm{M}$.

I then ran trials to measure whether the addition of $\mathrm{MgCl}$ affected the photophysiology of the Symbiodinium cells in the anemone. I did this by running rapid light curves (RLC) to measure effective PSII quantum yield (Y(II)), quantum yield of regulated energy dissipation (Y(NPQ)) and quantum yield of non-regulated energy dissipation (Y(NO)) in anemones bathed in $0.05 \mathrm{M} \mathrm{MgCl}$ or just in $\mathrm{FSW}$. The anemones were again attached in 3 $\mathrm{ml}$ tubes within a holder in the PAM. There were 4 replicate tests $(n=6)$. Anemones were placed in their treatment under $100( \pm 10) \mu \mathrm{mol}$ photons $\mathrm{m}^{-2} \mathrm{~s}^{-1}$ for 7 hours before the RLC. 

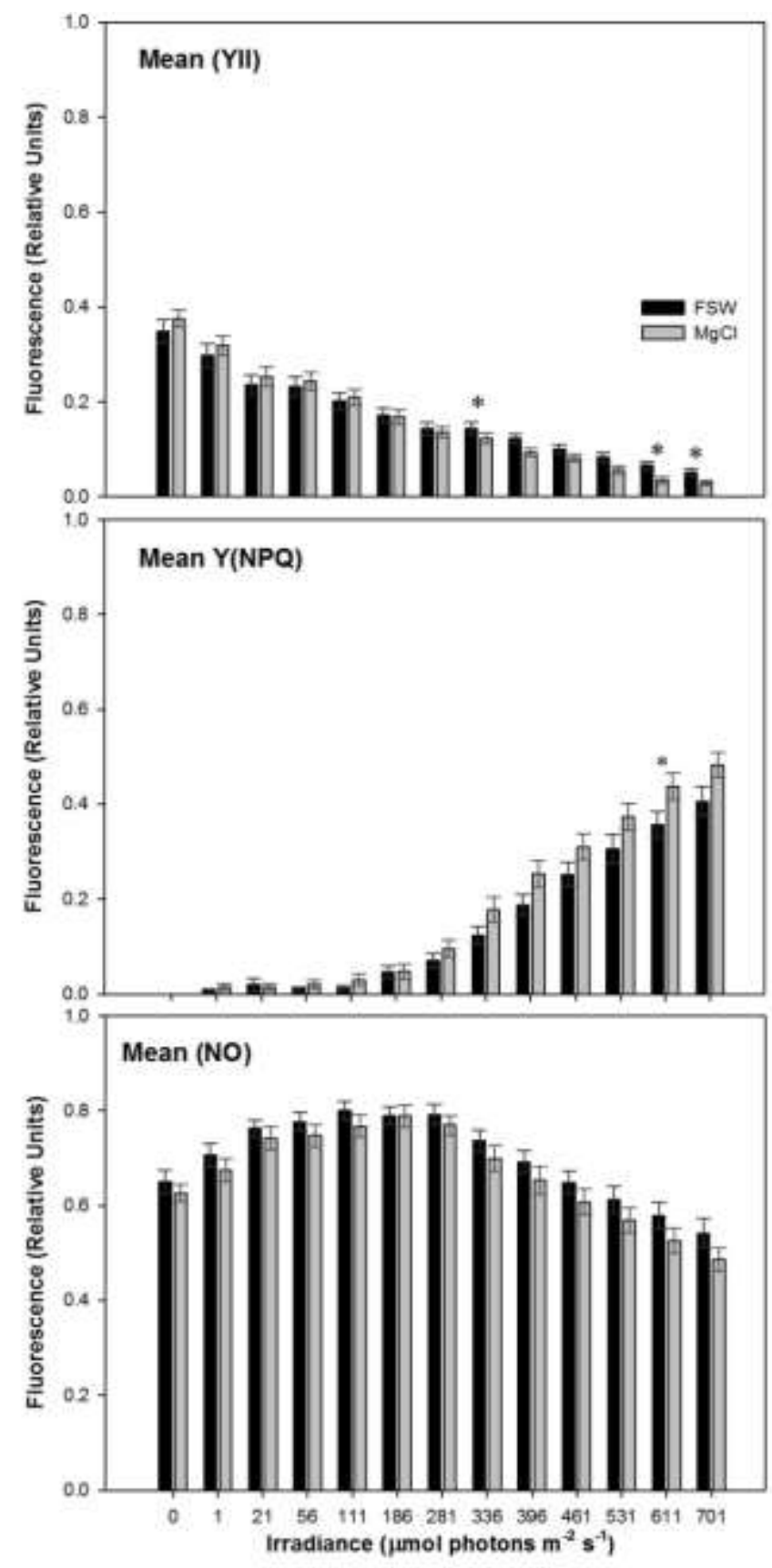

Figure A3.2.1: Mean ( $n=6 \pm S E)$ of $Y(I I)$, (YNPQ) and Y(NO) during four RLCs to test whether $0.05 \mathrm{M} \mathrm{MgCl}$ dissolved in FSW would affect the photophysiology of the Symbiodinium cells from within A. aureoradiata anemones. Asterisks mark significant differences between the means at specific irradiance levels $\left(^{*} \mathrm{p}<0.05\right.$, ANOVA, Tukey post hoc). 
The results showed that under the majority of irradiance levels there was no significant difference in means between anemones in FSW and those in $0.05 \mathrm{M} \mathrm{MgCl}(\mathrm{p}=0.05)$ (Figure A3.2.1). I concluded that using $\mathrm{MgCl}$ to relax anemones to stop them moving through the measurements of fluorescence induction kinetics was a viable option as the photophysiology of PSII of the anemones in FSW and $\mathrm{MgCl}$ reacted in a similar manner, and would not affect the induction kinetics for the DCMU tests.

I then re-ran tests to measure which concentration of DCMU could be used for the experiment. However, one of the tests was run with anemones in an unheated bath as the heater was on loan to another experiment. Even though the room temperature was not cold and would have been no more than $5^{\circ} \mathrm{C}$ below the previous tests, the anemones were all tightly closed. I decided that working with the whole anemone was proving to be too difficult and too varied. Controlling their behaviour, whilst not relaxing them too much or affecting their photophysiology was not possible. Therefore, further DCMU trials were run on Symbiodinium cells isolated from the anemones.

Four replicate tests were run with 25 anemones used in each. The anemones were broken up in a small liquidiser and the tissue then ground with a manual glass grinder to release the Symbiodinium cells from the animal cells. The cell suspension was spun five times in a centrifuge to separate the algal from the animal cells. The final supernatant was made up to $16 \mathrm{ml}$ with FSW and then pipetted into $1 \mathrm{ml}$ tubes. There were 4 treatments, a control with just FSW and 3 final concentrations of DCMU $(10 \mu \mathrm{M}, 20 \mu \mathrm{M}$ and $30 \mu \mathrm{M})$. Therefore there were 4 tubes in each treatment. The actinic light setting for the run was set at $396 \mu \mathrm{mol}$ photons $\mathrm{m}^{-2} \mathrm{~s}^{-1}$. As the addition of DCMU was manually carried out at a non-specific time, when the kinetic curve had settled, a graph could not be drawn with the mean outcome for the 4 treatments. The objective was to have a quick and steep curve after adding DCMU but not enough to cause loss of saturation pulses after its addition. After running the four replicates and visually looking at the effects of the different concentrations of DCMU, I decided to use $10 \mu \mathrm{M}$ concentration in the experiment planned (Figure A3.2.3) 

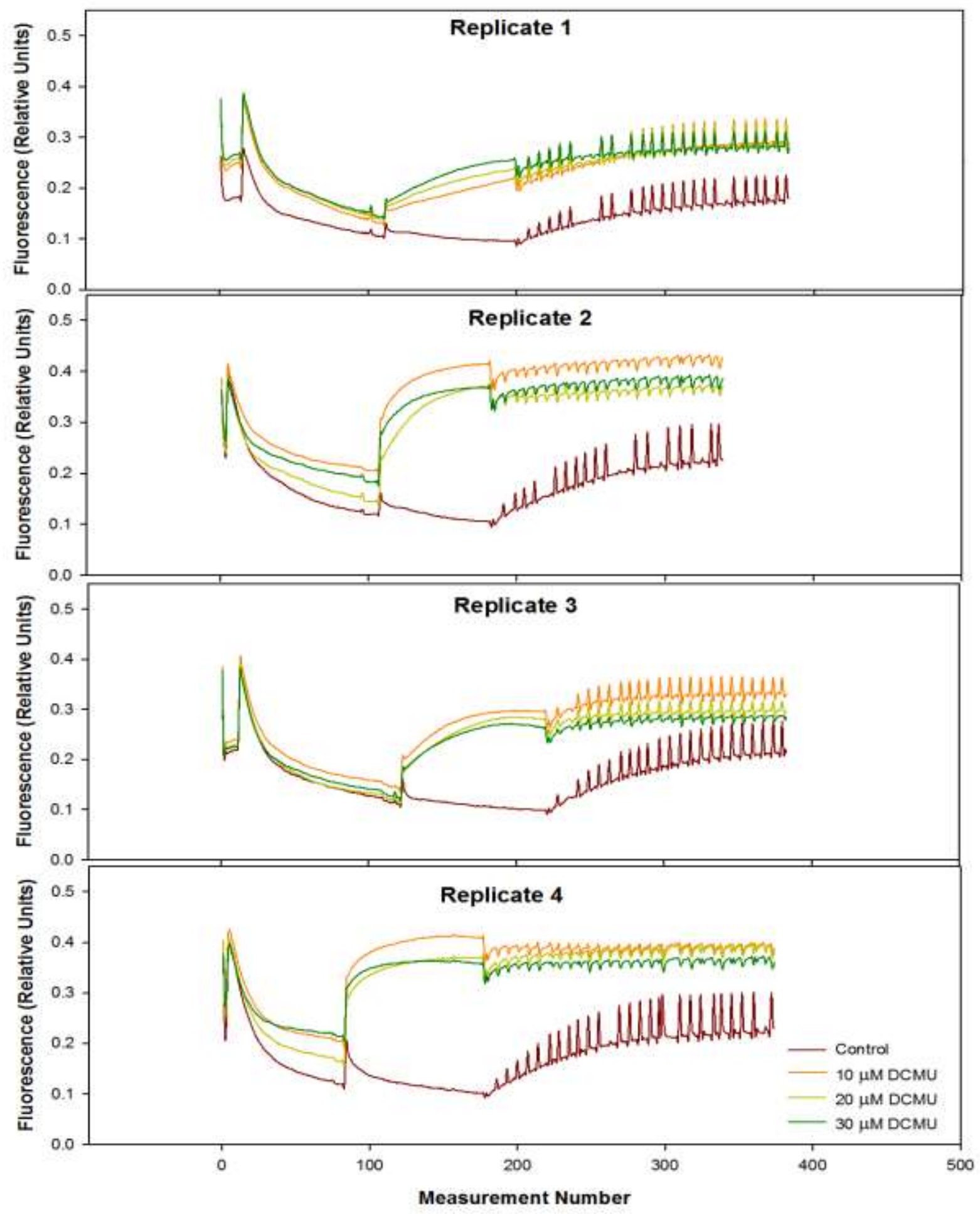

Figure A3.2.3: Chlorophyll fluorescence induction kinetics of the DCMU concentration trials on isolated Symbiodinium cells. Each line represents the mean $(n=4)$ of a treatment in the replicate. 
A $25 \mathrm{ml}$ DCMU solution was made up into a $1 \mathrm{mM}$ stock by firstly dissolving $0.0058 \mathrm{~g}$ of DCMU in $2 \mathrm{ml}$ of acetone and topped up to $25 \mathrm{ml}$ with FSW. Therefore the next trial run was to measure whether the acetone had any effect on the photophysiology of the Symbiodinium cells. To do this, another RLC trial was run with 3 replicates $(\mathrm{n}=3) .14$

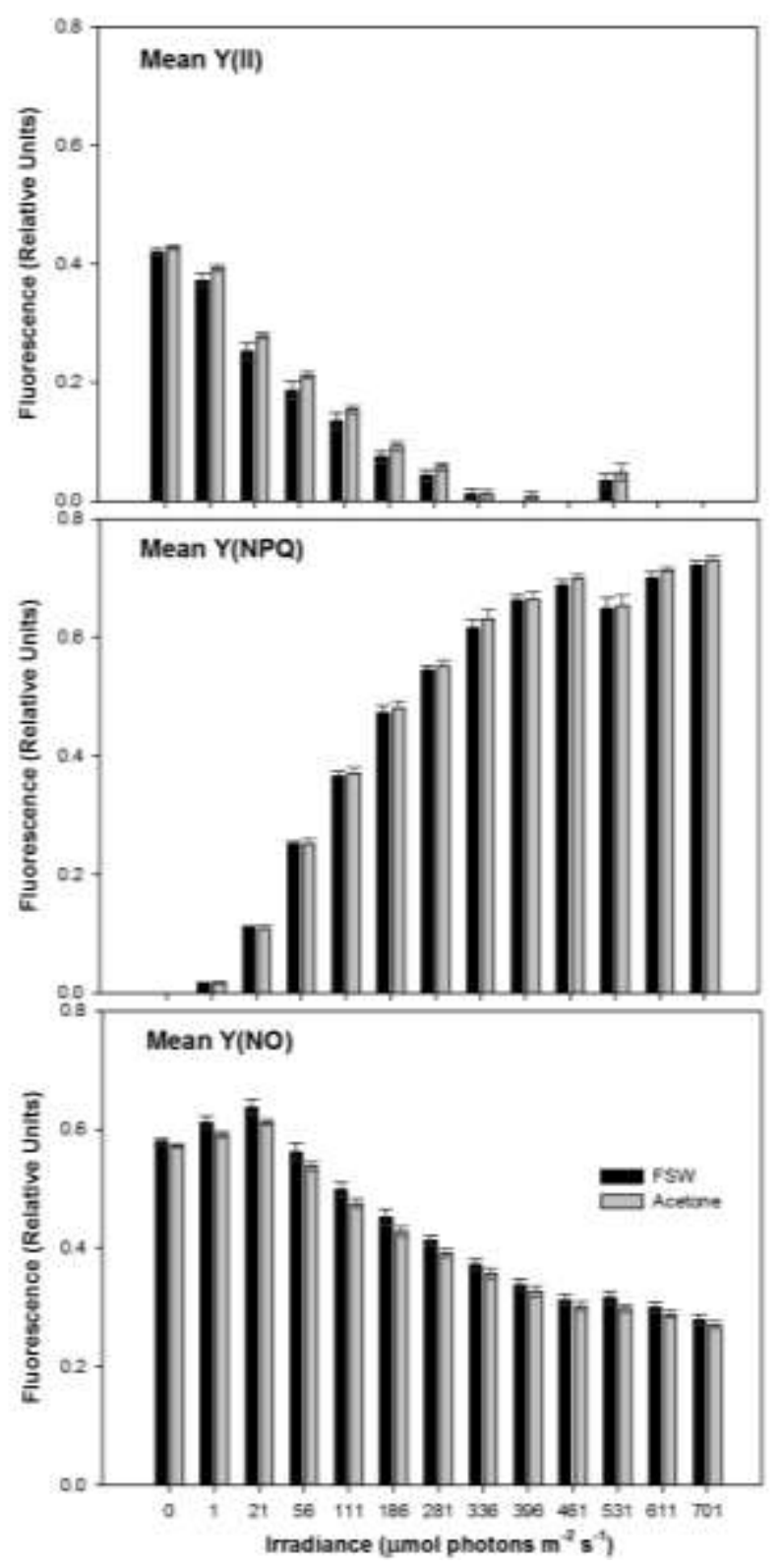
anemones were ground and the isolated Symbiodinium cells were suspended in 8 $\mathrm{ml}$ of FSW from which $990 \mu \mathrm{l}$ was then pipetted into each of the tubes for the PAM run. $10 \mu \mathrm{l}$ of FSW was pipetted into 3 tubes (Control) and $10 \mu 1$ of diluted acetone to the other 3 tubes. The diluted acetone came from stock of $2 \mathrm{ml}$ acetone with $23 \mathrm{ml} \mathrm{FSW}$. The tubes were then dark adapted for 20 minutes before the RLC was run. There were no significant differences in mean at any of the irradiance levels in any of the parameters measured. Therefore no significant effect of the acetone on the photophysiology of the Symbiodinium cells can be assumed. (Figure A3.2.4)

Figure A3.2.4: Acetone dilution tests (Mean, $n=3 \pm S E$ ) of $Y(I I)$, (YNPQ) and $Y(N O)$ during four RLCs to test whether acetone diluted in FSW would affect the photophysiology of the Symbiodinium cells within A. aureoradiata anemones. There were no significant differences in means at any irradiance levels $(\mathrm{p}=0.05)$. 
However, after all these trials there was still one problem that made running an experiment based on Hill et al's (2005) study unworkable. To be able to make any of the calculations measuring the three components of NPQ, the first saturation pulse spike had to be greater than any other fluorescence measurements made in the kinetic curve. In the majority of the trials this did not occur (Figure A3.2.5).

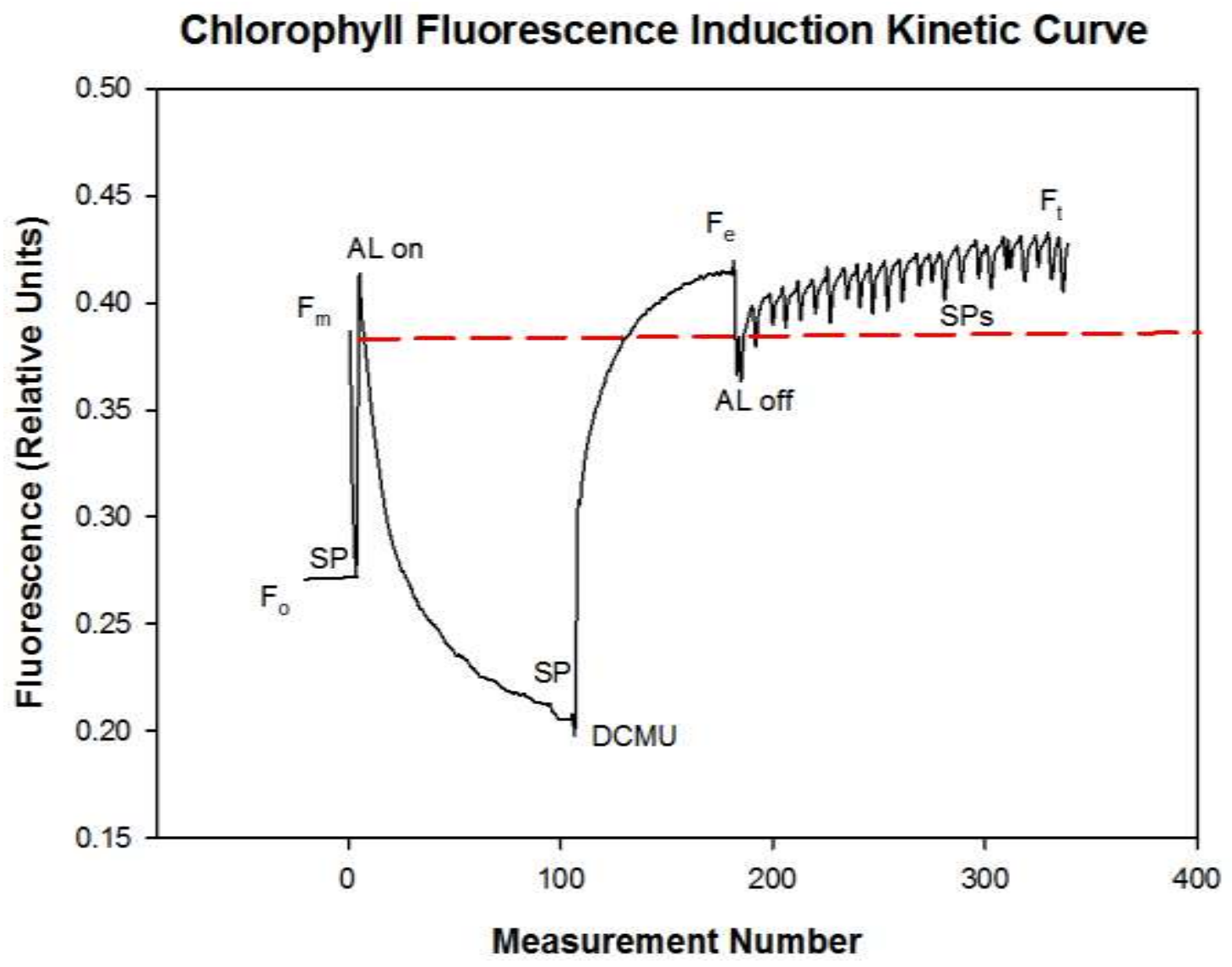

Figure A3.2.5: A chlorophyll fluorescence induction kinetic curve with $10 \mu \mathrm{M}$ DCMU added to a suspension of isolated Symbiodinium cells in FSW. This graph illustrates how the peak of the first saturation pulse, $\mathrm{F}_{\mathrm{m}}$, is lower than $\mathrm{F}_{\mathrm{e}}$ and $\mathrm{F}_{\mathrm{t}}$, thereby making any calculations to measure the components of NPQ unworkable. SP saturating pulse, $\mathrm{AL}$ - actinic light

A shorter saturation pulse than expected after dark adaptation suggests that the reaction centres in PSII are not all open. In other words all the electron in the primary quinone electron acceptor $\left(Q_{A}\right)$ have not moved on through PSII and onto PS I (Wykoff et al. 1998). Therefore, on exposing the PSII reaction centres to a saturation pulse, the difference in $\mathrm{F}_{\mathrm{m}}$ 
and $\mathrm{F}_{\mathrm{o}}$ is smaller and the saturation pulse looks shorter than if the centres had all been open. To 'empty' PSII a far-red light $(730 \mathrm{~nm})$ can be used, instead of keeping the samples in the dark prior to the first saturation pulse, so as to stimulate PSI and to increase the flow of electrons out of PSII (Wykoff et al. 1998)

A final trial was run exposing isolated Symbiodinium cells to far-red light to measure if there was an increase in the saturation pulse peak at the beginning of a test. Five tests were run $(\mathrm{n}=3)$. Symbiodinium cells were isolated, re-suspended and then pipetted into 6 tubes for the PAM. Three were placed in the dark (control) and 3 were placed under an illuminated far-red filter for the same amount of time. In each test the time under the light was increased, starting at 10 seconds and ending at 60 seconds. There was no difference between the dark adapted or the far-red stimulated suspension.

Six months of trials to run the experiment originally planned were not successful. Therefore I changed the objective in my photophysiology experiment. Instead of measuring the ratio of the three components of NPQ I would measure the total energy dissipated by NPQ compared to energy used for carbon fixation, and measure how well PSII down-regulated to decrease damage to the chloroplast by light. The experiment is described in Chapter 3. 


\section{APPENDIX 4 - Time trials to determine how long isolated Symbiodinium cells remained photosynthetically healthy}

The aim of these trials was to establish how long Symbiodinium cells isolated from $A$. aureoradiata would remain photosynthetically healthy and also the best time to start the photophysiology experiment. There were three trials.
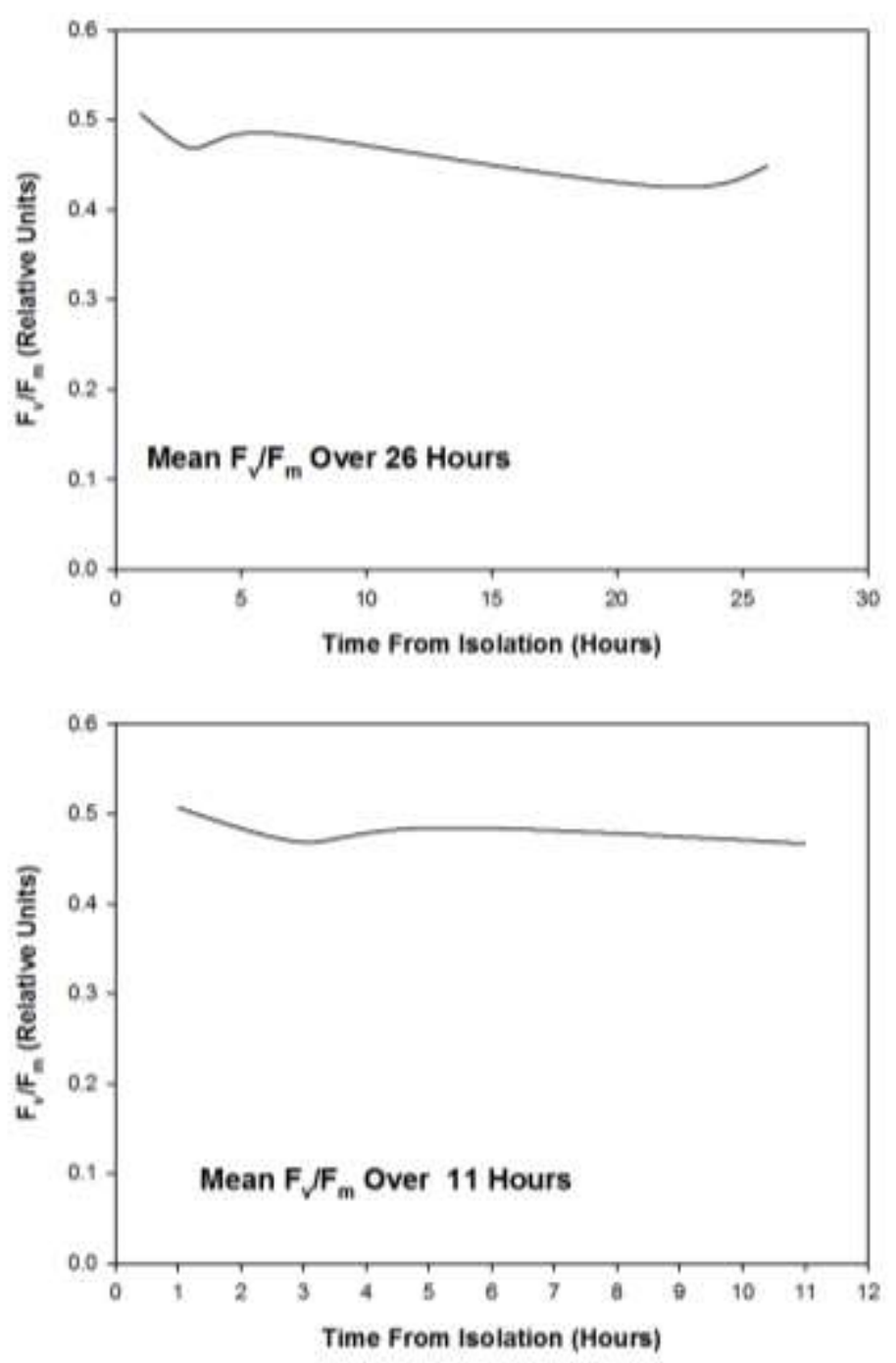

Twenty-five anemones were crushed and the Symbiodinium cells resuspended, after isolation, in $30 \mathrm{ml}$ of FSW. They were kept under $100 \mu \mathrm{mol}$ photon $\mathrm{m}^{-2} \mathrm{~s}^{-1}$ irradiance at $18{ }^{\circ} \mathrm{C}$. After settling for an hour, $2 \mathrm{ml}$ of suspension was removed and divided into 2 tubes, and dark adapted for 20 mins before measuring $\mathrm{F}_{\mathrm{v}} / \mathrm{F}_{\mathrm{m}}$. A further 5 measurements were spread over the next 25 hours (Figure A4.1).

Figure A4.1: Time trials to measure the photosynthetic health of freshly isolated Symbiodinium cells (mean, $\mathrm{n}=3$ ). The first graph shows measurements over the first 26 hours after isolation and the second shows measurements over the first 11 hours. 
The results indicated an immediate drop in photosynthetic health over the first three hours before a levelling out and maintenance of health to around 6 hours before $F_{v} / F_{m}$ gradually dropped away again. Based on these trials I let the isolated Symbiodinium cells rest for 3 hours in the main experiment before taking the first PAM measurements and separating the algal suspension between the six treatment tanks. I also kept the suspension in the treatments for only a further 6 hours to reduce bias in the final measurements of the experiment. It should be noted, however, that the change in $F_{v} / F_{m}$ over time is relatively small compared to the first measurement. 


\section{APPENDIX 5 - Cell Counts}

Table A5.1: Estimated density of cells in the algal suspension used in the photophysiology experiment. Counts were made using a haemocytometer (Improved Neubauer) at the beginning and at the end of the experiment. Six replicates of each sample were counted 4 times and the final mean count expressed per ml of cell suspension.

\begin{tabular}{|c|c|c|c|c|c|c|c|c|}
\hline \multicolumn{9}{|c|}{$\begin{array}{c}\text { No of Symbiodinium cells/ml of algal } \\
\text { suspension }\end{array}$} \\
\hline \multicolumn{2}{|r|}{ Treatment } & $\operatorname{Rep} 1$ & $\operatorname{Rep} 2$ & $\operatorname{Rep} 3$ & $\operatorname{Rep} 4$ & $\operatorname{Rep} 5$ & Mean & SE \\
\hline Beginning & $16^{\circ} \mathrm{C}$ Low & 2371667 & 2910000 & 2565833 & 2573333 & 2692778 & 2622722 & 88357 \\
\hline \multirow{6}{*}{ 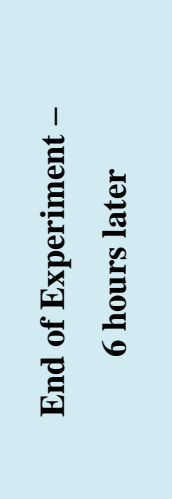 } & $8{ }^{\circ} \mathrm{C}$ Low & 1862500 & 2165833 & 1906667 & 1310000 & 2342500 & 1917500 & 175284 \\
\hline & $8^{\circ} \mathrm{C}$ High & 1607500 & 2245833 & 1742500 & 1897500 & 1802500 & 1859167 & 107503 \\
\hline & $16^{\circ} \mathrm{C}$ Low & 1484167 & 1585833 & 1990000 & 1664167 & 1621667 & 1669167 & 85553 \\
\hline & $16^{\circ} \mathrm{C}$ High & 1815833 & 2234167 & 2013333 & 1640000 & 1502500 & 1841167 & 130364 \\
\hline & $30{ }^{\circ} \mathrm{C}$ Low & 1787500 & 2510000 & 2277500 & 2010000 & 1606667 & 2038333 & 162746 \\
\hline & $30^{\circ} \mathrm{C}$ High & 1890833 & 2155000 & 2517500 & 1889167 & 1755000 & 2041500 & 135545 \\
\hline
\end{tabular}




\section{A5.1 Statistical results for cell density}

Table A5.1.1: PASW output for mean cell density $(n=24 \pm$ SE) using ANOVA where normality and variance had been assumed. Significance is assumed when $\mathrm{p}<0.05$.

Tests of Between-Subjects Effects

Dependent Variable: Cell Count

\begin{tabular}{|l|r|r|r|r|r|}
\hline Source & $\begin{array}{c}\text { Type III Sum of } \\
\text { Squares }\end{array}$ & df & Mean Square & F & p value. \\
\hline Corrected Model & $2.075 \mathrm{E} 12$ & 6 & $3.458 \mathrm{E} 11$ & 4.770 & 0.003 \\
Intercept & $1.047 \mathrm{E} 14$ & 1 & $1.047 \mathrm{E} 14$ & 1444.296 & $<0.001$ \\
Treatment & $2.075 \mathrm{E} 12$ & 6 & $3.458 \mathrm{E} 11$ & 4.770 & 0.003 \\
Error & $1.522 \mathrm{E} 12$ & 21 & $7.248 \mathrm{E} 10$ & & \\
Total & $1.083 \mathrm{E} 14$ & 28 & & & \\
Corrected Total & $3.597 \mathrm{E} 12$ & 27 & & & \\
\hline
\end{tabular}

R Squared $=.577($ Adjusted R Squared $=.456)$

Table A5.1.2: Post hoc tests using Tukey HSD, comparing the difference in mean $(n=24 \pm$ SE) of the cell density in each treatment $(n=6)$ between the start and finish for each treatment and between each of the treatments at the end of the experiment.

\begin{tabular}{|c|c|c|c|c|c|c|c|}
\hline & Start & L8 & L16 & L30 & H8 & H16 & H30 \\
\hline Start & & 0.021 & 0.003 & 0.044 & 0.007 & 0.006 & 0.117 \\
\hline L8 & 0.021 & & 0.973 & 1.000 & 0.999 & 0.996 & 0.979 \\
\hline L16 & 0.003 & 0.973 & & 0.882 & 1.000 & 1.000 & 0.625 \\
\hline L30 & 0.044 & 1.000 & 0.882 & & 0.979 & 0.963 & 0.999 \\
\hline H8 & 0.007 & 0.999 & 1.000 & 0.979 & & 1.000 & 0.837 \\
\hline H16 & 0.006 & 0.996 & 1.000 & 0.963 & 1.000 & & 0.786 \\
\hline H30 & 0.117 & 0.979 & 0.625 & 0.999 & 0.837 & 0.786 & \\
\hline
\end{tabular}




\section{APPENDIX 6- Statistical Data for the Results in Chapter 3}

The following data originates from statistical analyses using PASW (version 18.00). Variation of fluorescence measurements were tested using a repeat measure one-way analysis of variance (rmANOVA, $\alpha=0.05$ ) with time of measurements as the withinsubjects factor and light and temperature as the between-subjects factors. Where assumptions of normality and equal variance were not satisfied, transformation of data were carried out using an arcsin or a reflect and square root method. The Mauchley Sphericity Test was used in all analyses to test for sphericity of data. As all data met assumptions of sphericity, post hoc tests were carried out using Tukey HSD.

\section{$\underline{\text { A6.1: } \text { Statistical Results for } \mathbf{F}_{\mathrm{v}}} \underline{\underline{\mathbf{F}}}$}

Table A6.1.1: PASW output for mean $(n=4 \pm S E)$ arcsin transformed $F_{v} / F_{m}$ using rmANOVA where normality, variance and sphericity had been assumed. Significance is assumed when $\mathrm{p}<0.05$.

\section{Tests of Within-Subjects Effects}

Measure:Arcsin $\mathrm{F}_{\mathrm{v}} / \mathrm{F}_{\mathrm{m}}$

\begin{tabular}{|l|r|r|r|r|r|}
\hline Source & Type III Sum of Squares & df & Mean Square & \multicolumn{1}{|c|}{ F } & \multicolumn{1}{c|}{ p value } \\
\hline Time & 0.389 & 3 & 0.130 & 228.831 & $<0.001$ \\
Time * Light & 0.016 & 3 & 0.005 & 9.418 & $<0.001$ \\
Time * Temp & 0.433 & 6 & 0.072 & 127.476 & $<0.001$ \\
Time * Light * Temp & 0.004 & 6 & 0.001 & 1.108 & 0.370 \\
Error(Time) & 0.031 & 54 & 0.001 & & \\
\hline
\end{tabular}


Table A6.1.2: PASW output for mean $(n=4 \pm S E)$ arcsin transformed $F_{v} / F_{m}$ tests of within-subjects contrasts to find the time-points between which there had been significant effects of factor interactions with time. Significance is assumed when $\mathrm{p}<0.05$.

\section{Tests of Within-Subjects Contrasts}

\begin{tabular}{|c|c|c|c|c|c|c|}
\hline Source & Time & $\begin{array}{c}\text { Type III Sum of } \\
\text { Squares }\end{array}$ & df & Mean Square & $\mathrm{F}$ & $\mathrm{p}$ value \\
\hline \multirow[t]{3}{*}{ Time } & Start vs. $2 \mathrm{~h}$ & 0.501 & 1 & 0.501 & 552.013 & $<0.001$ \\
\hline & $2 \mathrm{~h}$ vs. $4 \mathrm{~h}$ & 0.001 & 1 & 0.001 & 1.579 & 0.225 \\
\hline & $4 \mathrm{~h}$ vs. $6 \mathrm{~h}$ & 0.002 & 1 & 0.002 & 1.096 & 0.309 \\
\hline \multirow[t]{3}{*}{ Time * Light } & Start vs. $2 \mathrm{~h}$ & 0.018 & 1 & 0.018 & 19.474 & $<0.001$ \\
\hline & $2 \mathrm{~h}$ vs. $4 \mathrm{~h}$ & 0.002 & 1 & 0.002 & 2.596 & 0.125 \\
\hline & $4 \mathrm{~h}$ vs. $6 \mathrm{~h}$ & 0.007 & 1 & 0.007 & 4.648 & 0.045 \\
\hline \multirow[t]{3}{*}{ Time $*$ Temp } & Start vs. $2 \mathrm{~h}$ & 0.366 & 2 & 0.183 & 201.725 & $<0.001$ \\
\hline & $2 \mathrm{~h}$ vs. $4 \mathrm{~h}$ & 0.027 & 2 & 0.014 & 15.702 & $<0.001$ \\
\hline & $4 \mathrm{~h}$ vs. $6 \mathrm{~h}$ & 0.005 & 2 & 0.002 & 1.611 & 0.227 \\
\hline \multirow[t]{3}{*}{ Time $*$ Light $*$ Temp } & Start vs. $2 \mathrm{~h}$ & 0.001 & 2 & 0.000 & 0.471 & 0.632 \\
\hline & $2 \mathrm{~h}$ vs. $4 \mathrm{~h}$ & 0.001 & 2 & 0.001 & 0.582 & 0.569 \\
\hline & $4 \mathrm{~h}$ vs. $6 \mathrm{~h}$ & 0.002 & 2 & 0.001 & 0.624 & 0.547 \\
\hline \multirow[t]{3}{*}{ Error(Time) } & Start vs. $2 \mathrm{~h}$ & 0.016 & 18 & 0.001 & & \\
\hline & $2 \mathrm{~h}$ vs. $4 \mathrm{~h}$ & 0.016 & 18 & 0.001 & & \\
\hline & $4 \mathrm{~h}$ vs. $6 \mathrm{~h}$ & 0.027 & 18 & 0.001 & & \\
\hline
\end{tabular}


Table A6.1.3: PASW output for mean $(n=4 \pm S E)$ arcsin transformed $F_{v} / F_{m}$ tests of between-subjects to find out if the two factors and their interaction had a significant effect on the experiment outcome. Significance is assumed when $\quad \mathrm{p}<0.05$.

\section{Tests of Between-Subjects Effects}

Measure:MEASURE_1Arcsin $\mathrm{F}_{v} / \mathrm{F}_{\mathrm{m}}$

Transformed Variable:Average

\begin{tabular}{|l|r|r|r|r|r|}
\hline Source & $\begin{array}{c}\text { Type III Sum of } \\
\text { Squares }\end{array}$ & df & Mean Square & F & p value \\
\hline Intercept & 12.342 & 1 & 12.342 & 20631.052 & $<0.001$ \\
Light & 0.038 & 1 & 0.038 & 62.743 & $<0.001$ \\
Temp & 1.213 & 2 & 0.607 & 1014.060 & $<0.001$ \\
Light * Temp & 0.005 & 2 & 0.002 & 3.865 & 0.040 \\
Error & 0.011 & 18 & 0.001 & & \\
\hline
\end{tabular}

Table A6.1.4: Post hoc tests using Tukey HSD, as assumptions of sphericity were assumed, comparing the difference in mean $(n=4 \pm S E)$ of $\arcsin F_{v} / F_{m}$ in each treatment between the time-points in a rmANOVA analysis. Significance is assumed when $\mathrm{p}<0.05$.

\begin{tabular}{|c|c|c|c|c|c|}
\hline \multirow{4}{*}{ 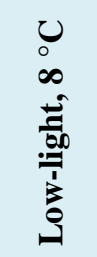 } & & Start & 2 hours & 4 hours & 6 hours \\
\hline & Start & & $<0.001$ & $<0.001$ & $<0.001$ \\
\hline & 2 hours & $<0.001$ & & 0.065 & 0.281 \\
\hline & 4 hours & $<0.001$ & 0.065 & & 0.795 \\
\hline 6 & & Start & 2 hours & 4 hours & 6 hours \\
\hline 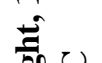 & Start & & 0.103 & 0.053 & 0.513 \\
\hline 管。 & 2 hours & 0.103 & & 0.978 & 0.682 \\
\hline & 4 hours & 0.053 & 0.978 & & 0.456 \\
\hline 尺 & & Start & 2 hours & 4 hours & 6 hours \\
\hline 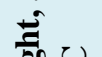 & Start & & 0.205 & 0.571 & 0.342 \\
\hline${ }^{\circ}$ & 2 hours & 0.205 & & 0.856 & 0.011 \\
\hline$\beth$ & 4 hours & 0.571 & 0.856 & & 0.042 \\
\hline
\end{tabular}


Table 6.1.4 continued: Post hoc tests using Tukey HSD of arcsin Fv/Fm in each treatment between the timepoints in a rmANOVA analysis. Significance is assumed when $\mathrm{p}<0.05$.

\begin{tabular}{|c|c|c|c|c|c|}
\hline \multirow{4}{*}{ 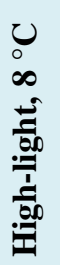 } & & Start & 2 hours & 4 hours & 6 hours \\
\hline & Start & & $<0.001$ & $<0.001$ & $<0.001$ \\
\hline & 2 hours & $<0.001$ & & 0.057 & 0.001 \\
\hline & 4 hours & $<0.001$ & 0.057 & & 0.140 \\
\hline$\bigcup_{0}$ & & Start & 2 hours & 4 hours & 6 hours \\
\hline$=$ & Start & & $<0.001$ & $<0.001$ & $<0.001$ \\
\hline$\frac{\overline{0}}{7}$ & 2 hours & $<0.001$ & & 0.969 & 0.909 \\
\hline 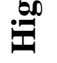 & 4 hours & $<0.001$ & 0.969 & & 0.996 \\
\hline$\bigcup_{0}$ & & Start & 2 hours & 4 hours & 6 hours \\
\hline ले & Start & & 0.035 & 0.767 & 0.951 \\
\hline$\frac{500}{100}$ & 2 hours & 0.035 & & 0.177 & 0.086 \\
\hline 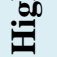 & 4 hours & 0.767 & 0.177 & & 0.969 \\
\hline
\end{tabular}

Table A6.1.5: Post hoc tests using Tukey HSD, as assumptions of sphericity were assumed, comparing the difference in mean $(n=4 \pm S E)$ of $\arcsin F_{v} / F_{m}$ at 6 hours with an ANOVA analysis. Significance is assumed when $\mathrm{p}<0.05$.

\begin{tabular}{|c|c|c|c|c|c|c|}
\hline & L8 & L16 & L30 & H8 & H16 & H30 \\
\hline L8 & & $<0.001$ & $<0.001$ & $<0.001$ & $<0.001$ & $<0.001$ \\
\hline L16 & $<0.001$ & & 0.307 & $<0.001$ & 0.009 & 0.999 \\
\hline $\mathbf{L 3 0}$ & $<0.001$ & 0.307 & & $<0.001$ & $<0.001$ & 0.492 \\
\hline $\mathbf{H 8}$ & $<0.001$ & $<0.001$ & $<0.001$ & & $<0.001$ & $<0.001$ \\
\hline $\mathbf{H 1 6}$ & $<0.001$ & 0.009 & $<.001$ & $<0.001$ & & 0.004 \\
\hline $\mathbf{H 3 0}$ & $<0.001$ & 0.999 & 0.492 & $<0.001$ & 0.004 & \\
\hline
\end{tabular}




\section{$\underline{\text { A6.2 Statistical results for } \Delta \mathbf{F}} \underline{\underline{F}} \underline{\underline{m}} \underline{\text { from the dark-induction curve }}$}

Table A6.2.1: Post hoc tests using Tukey HSD, as assumptions of sphericity were assumed, comparing the difference in mean $(n=4 \pm \mathrm{SE})$ of the final measurement of $\Delta \mathrm{F}_{\mathrm{v}} / \mathrm{F}_{\mathrm{m}}$ ' at 6 hours with an ANOVA analysis. Significance is assumed when $\mathrm{p}<0.05$.

\begin{tabular}{|c|c|c|c|c|c|c|}
\hline & L8 & L16 & L30 & H8 & H16 & H30 \\
\hline $\mathbf{L 8}$ & & $<0.001$ & $<0.001$ & 0.005 & 0.005 & 0.156 \\
\hline $\mathbf{L 1 6}$ & $<0.001$ & & $<0.001$ & $<0.001$ & $<0.001$ & $<0.001$ \\
\hline $\mathbf{L 3 0}$ & $<0.001$ & $<0.001$ & & $<0.001$ & $<0.001$ & $<0.001$ \\
\hline $\mathbf{H 8}$ & 0.005 & $<0.001$ & $<0.001$ & & 1.000 & 0.535 \\
\hline $\mathbf{H 1 6}$ & 0.005 & $<0.001$ & $<.001$ & 1.000 & & 0.535 \\
\hline $\mathbf{H 3 0}$ & 0.156 & $<0.001$ & $<0.001$ & 0.535 & 0.535 & \\
\hline
\end{tabular}

Table A6.2.2: Mean and standard error of the last $\Delta \mathrm{F}_{\mathrm{v}} / \mathrm{F}_{\mathrm{m}}$ ' of each treatment at each time-point

\begin{tabular}{|c|c|c|c|c|c|c|c|}
\hline \multirow{5}{*}{ 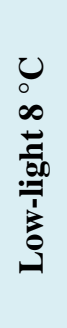 } & & Mean & St Error & \multirow{5}{*}{ 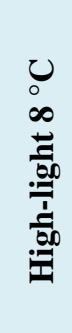 } & & Mean & St Error \\
\hline & Start & 0.1859 & 0.0109 & & Start & 0.1859 & 0.0109 \\
\hline & 2 hours & 0.0615 & 0.0056 & & 2 hours & 0.0755 & 0.0755 \\
\hline & 4 hours & 0.0541 & 0.0064 & & 4 hours & 0 & 0 \\
\hline & 6 hours & 0.0650 & 0.0144 & & 6 hours & 0 & 0 \\
\hline \multirow{5}{*}{ 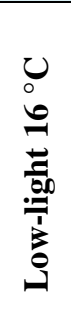 } & & Mean & St Error & \multirow{5}{*}{ 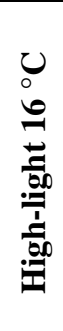 } & & Mean & St Error \\
\hline & Start & 0.1859 & 0.0109 & & Start & 0.1859 & 0.0109 \\
\hline & 2 hours & 0.1063 & 0.0056 & & 2 hours & 0 & 0 \\
\hline & 4 hours & 0.1120 & 0.0271 & & 4 hours & 0 & 0 \\
\hline & 6 hours & 0.1516 & 0.0208 & & 6 hours & 0 & 0 \\
\hline \multirow{5}{*}{ 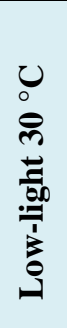 } & & Mean & St Error & \multirow{5}{*}{ 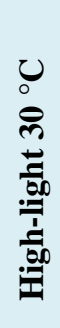 } & & Mean & St Error \\
\hline & Start & 0.1859 & 0.0109 & & Start & 0.1859 & 0.0109 \\
\hline & 2 hours & 0.2082 & 0.0127 & & 2 hours & 0 & 0 \\
\hline & 4 hours & 0.2562 & 0.01 & & 4 hours & 0.01 & 0.0081 \\
\hline & 6 hours & 0.2956 & 0.0031 & & 6 hours & 0.026 & 0.006 \\
\hline
\end{tabular}




\section{A6.3 Statistical results for the coefficient of non-photochemical quenching}

$\underline{(\mathrm{qN})}$

Table A6.3.1: Post hoc tests using Tukey HSD, as assumptions of sphericity were assumed, comparing the difference in mean $(n=4 \pm S E)$ of the final measurement of $\mathrm{qN}$ in each treatment between the time-points in a rmANOVA analysis. Significance is assumed when $\mathrm{p}<0.05$.

\begin{tabular}{|c|c|c|c|c|c|}
\hline \multirow{4}{*}{ 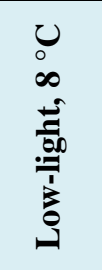 } & & Start & 2 hours & 4 hours & 6 hours \\
\hline & Start & & 0.273 & 0.894 & 0.775 \\
\hline & 2 hours & 0.273 & & 0.632 & 0.059 \\
\hline & 4 hours & 0.894 & 0.632 & & 0.384 \\
\hline \multirow{4}{*}{ 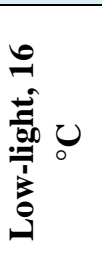 } & & Start & 2 hours & 4 hours & 6 hours \\
\hline & Start & & 0.006 & 0.05 & 0.895 \\
\hline & 2 hours & 0.006 & & 0.606 & 0.019 \\
\hline & 4 hours & 0.05 & 0.606 & & 0.161 \\
\hline \multirow{4}{*}{ 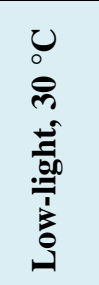 } & & Start & 2 hours & 4 hours & 6 hours \\
\hline & Start & & 0.016 & $<0.001$ & $<0.001$ \\
\hline & 2 hours & 0.016 & & 0.062 & $<0.001$ \\
\hline & 4 hours & $<0.001$ & 0.062 & & 0.015 \\
\hline \multirow{4}{*}{ 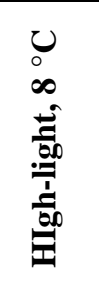 } & & Start & 2 hours & 4 hours & 6 hours \\
\hline & Start & & $<0.001$ & $<0.001$ & $<0.001$ \\
\hline & 2 hours & $<0.001$ & & 0.842 & 0.196 \\
\hline & 4 hours & $<0.001$ & 0.842 & & 0.572 \\
\hline \multirow{4}{*}{ 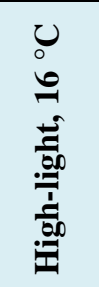 } & & Start & 2 hours & 4 hours & 6 hours \\
\hline & Start & & $<0.001$ & $<0.001$ & $<0.001$ \\
\hline & 2 hours & $<0.001$ & & 0.998 & 0.918 \\
\hline & 4 hours & $<0.001$ & 0.998 & & 0.967 \\
\hline \multirow{4}{*}{ 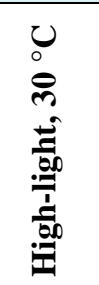 } & & Start & 2 hours & 4 hours & 6 hours \\
\hline & Start & & $<0.001$ & $<0.001$ & $<0.001$ \\
\hline & 2 hours & $<0.001$ & & 0.367 & 0.039 \\
\hline & 4 hours & $<0.001$ & 0.367 & & 0.510 \\
\hline
\end{tabular}


Table A6.3.2: Post hoc tests using Tukey HSD, as assumptions of sphericity were assumed, comparing the difference in mean $(\mathrm{n}=4 \pm \mathrm{SE})$ of the final measurement of $\mathrm{qN}$ at 6 hours with an ANOVA analysis. Significance is assumed when $\mathrm{p}<0.05$.

\begin{tabular}{|c|c|c|c|c|c|c|}
\hline & L8 & L16 & L30 & H8 & H16 & H30 \\
\hline L8 & & 0.787 & $<0.001$ & 0.005 & 0.005 & 0.002 \\
\hline L16 & 0.787 & & $<0.001$ & $<0.001$ & $<0.001$ & $<0.035$ \\
\hline L30 & $<0.001$ & $<0.001$ & & $<0.001$ & $<0.001$ & $<0.001$ \\
\hline H8 & $<0.001$ & $<0.001$ & $<0.001$ & & 0.973 & 0.122 \\
\hline H16 & $<0.001$ & $<0.001$ & $<.001$ & 0.973 & & 0.399 \\
\hline H30 & 0.002 & 0.035 & $<0.001$ & 0.122 & 0.399 & \\
\hline
\end{tabular}

\section{A6.4: Mean and $S E$ of $F_{0} \underline{F_{m}} \underline{\text { and } F_{t}}$}

Table 6.4.1: Mean $(n=4)$ and standard error of dark-adapted $F_{o}$, and $F_{m}$ and the last measurement of $F_{t}$ from each treatment at each time-point during a dark-light induction curve.

\begin{tabular}{|c|c|c|c|c|c|c|c|}
\hline \multirow[t]{2}{*}{ Treatment } & \multirow{2}{*}{$\begin{array}{c}\text { Time } \\
\text { (Hours) }\end{array}$} & \multicolumn{2}{|c|}{$\mathbf{F}_{\mathbf{o}}$} & \multicolumn{2}{|c|}{$\mathbf{F}_{\mathbf{m}}$} & \multicolumn{2}{|c|}{$\mathbf{F}_{\mathbf{t}}$} \\
\hline & & Mean & SE & Mean & SE & Mean & SE \\
\hline \multirow{4}{*}{ 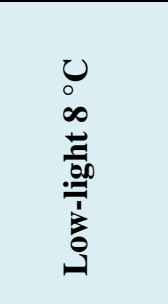 } & 0 & 0.289 & 0.0016 & 0.5276 & 0.009 & 0.1856 & 0.0016 \\
\hline & 2 & 0.1781 & 0.0073 & 0.2253 & 0.01 & 0.1094 & 0.0053 \\
\hline & 4 & 0.1564 & 0.0071 & 0.1774 & 0.0083 & 0.1120 & 0.0035 \\
\hline & 6 & 0.15 & 0.0073 & 0.1739 & 0.0085 & 0.1140 & 0.0044 \\
\hline \multirow{4}{*}{ 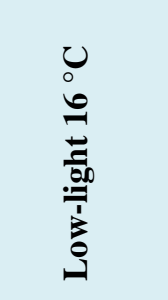 } & 0 & 0.289 & 0.0016 & 0.5276 & 0.009 & 0.1856 & 0.0016 \\
\hline & 2 & 0.2541 & 0.0049 & 0.4407 & 0.0109 & 0.1258 & 0.0029 \\
\hline & 4 & 0.241 & 0.0068 & 0.4142 & 0.0102 & 0.1462 & 0.0154 \\
\hline & 6 & 0.2389 & 0.0061 & 0.4232 & 0.0057 & 0.1486 & 0.0075 \\
\hline
\end{tabular}


Table 6.4.1 (cont'd): Mean ( $n=4)$ and standard error of dark-adapted $F_{o}$, and $F_{m}$ and the last measurement of $\mathrm{F}_{\mathrm{t}}$ from each treatment at each time-point during a dark-light induction curve.

\begin{tabular}{|c|c|c|c|c|c|c|c|}
\hline \multirow[t]{2}{*}{ Treatment } & \multirow{2}{*}{$\begin{array}{c}\text { Time } \\
\text { (Hours) }\end{array}$} & \multicolumn{2}{|c|}{$\mathbf{F}_{\mathbf{o}}$} & \multicolumn{2}{|c|}{$\mathbf{F}_{\mathbf{m}}$} & \multicolumn{2}{|c|}{$\mathbf{F}_{\mathbf{t}}$} \\
\hline & & Mean & SE & Mean & SE & Mean & SE \\
\hline \multirow{4}{*}{ 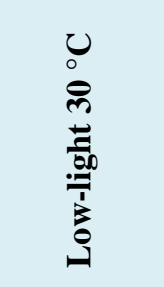 } & 0 & 0.289 & 0.0016 & 0.5276 & 0.009 & 0.1856 & 0.0016 \\
\hline & 2 & 0.3104 & 0.0045 & 0.5447 & 0.0104 & 0.2427 & 0.0132 \\
\hline & 4 & 0.3085 & 0.0072 & 0.554 & 0.0083 & 0.2972 & 0.0134 \\
\hline & 6 & 0.31 & 0.016 & 0.5828 & 0.0248 & 0.3133 & 0.0118 \\
\hline \multirow{4}{*}{ 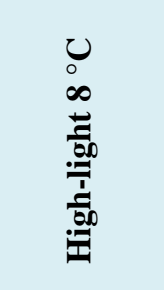 } & $\mathbf{0}$ & 0.289 & 0.0016 & 0.5276 & 0.009 & 0.1856 & 0.0016 \\
\hline & 2 & 0.1639 & 0.0032 & 0.2113 & 0.0267 & 0.0826 & 0.0017 \\
\hline & 4 & 0.1431 & 0.0031 & 0.155 & 0.0047 & 0.07732 & 0.0019 \\
\hline & 6 & 0.1323 & 0.00866 & 0.1376 & 0.0095 & 0.0757 & 0.0048 \\
\hline \multirow{4}{*}{ 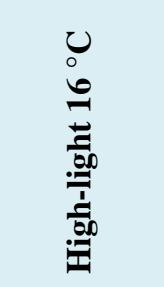 } & $\mathbf{0}$ & 0.289 & 0.0016 & 0.5276 & 0.009 & 0.1856 & 0.0016 \\
\hline & 2 & 0.2481 & 0.0013 & 0.3865 & 0.0037 & 0.0861 & 0.0033 \\
\hline & 4 & 0.2455 & 0.0055 & 0.387 & 0.0131 & 0.0875 & 0.0027 \\
\hline & 6 & 0.2428 & 0.0062 & 0.3841 & 0.0082 & 0.0903 & 0.0058 \\
\hline \multirow{4}{*}{ 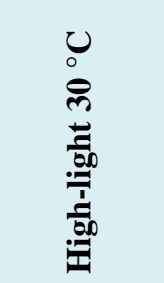 } & 0 & 0.289 & 0.0016 & 0.5276 & 0.009 & 0.1856 & 0.0016 \\
\hline & 2 & 0.2905 & 0.0047 & 0.4766 & 0.015 & 0.1073 & 0.0028 \\
\hline & 4 & 0.303 & 0.007 & 0.5221 & 0.0162 & 0.1314 & 0.0119 \\
\hline & 6 & 0.281 & 0.0092 & 0.5035 & 0.0196 & 0.0137 & 0.0112 \\
\hline
\end{tabular}




\section{A6.5: Statistical results for rETR $_{\max }$}

Table A6.5.1: PASW output for mean $\left(n=4 \pm\right.$ SE) reflect and square root transformed $\mathrm{rETR}_{\max }$ using rmANOVA where normality, variance and sphericity had been assumed. Significance is assumed when $\mathrm{p}<0.05$.

Tests of Within-Subjects Effects

Measure:MEASURE_1
\begin{tabular}{|l|r|r|r|r|r|}
\hline Source & Type III Sum of Squares & df & Mean Square & F & p value \\
\hline Time & 16.867 & 3 & 5.622 & 143.277 & $<0.001$ \\
Time * Light & 3.743 & 3 & 1.248 & 31.796 & $<0.001$ \\
Time * Temp & 28.126 & 6 & 4.688 & 119.457 & $<0.001$ \\
Time * Light * Temp & 0.737 & 6 & 0.123 & 3.132 & 0.010 \\
Error(Time) & 2.119 & 54 & 0.039 & & \\
\hline
\end{tabular}

Table A6.5.2: PASW output for mean $\left(n=4 \pm\right.$ SE) reflect and square root transformed $\mathrm{rETR}_{\max }$ tests of within-subjects contrasts to find the time-points between which there had been significant effects of factor interactions with time. Significance is assumed when $\mathrm{p}<0.05$.

Tests of Within-Subjects Contrasts

Measure:MEASURE_1

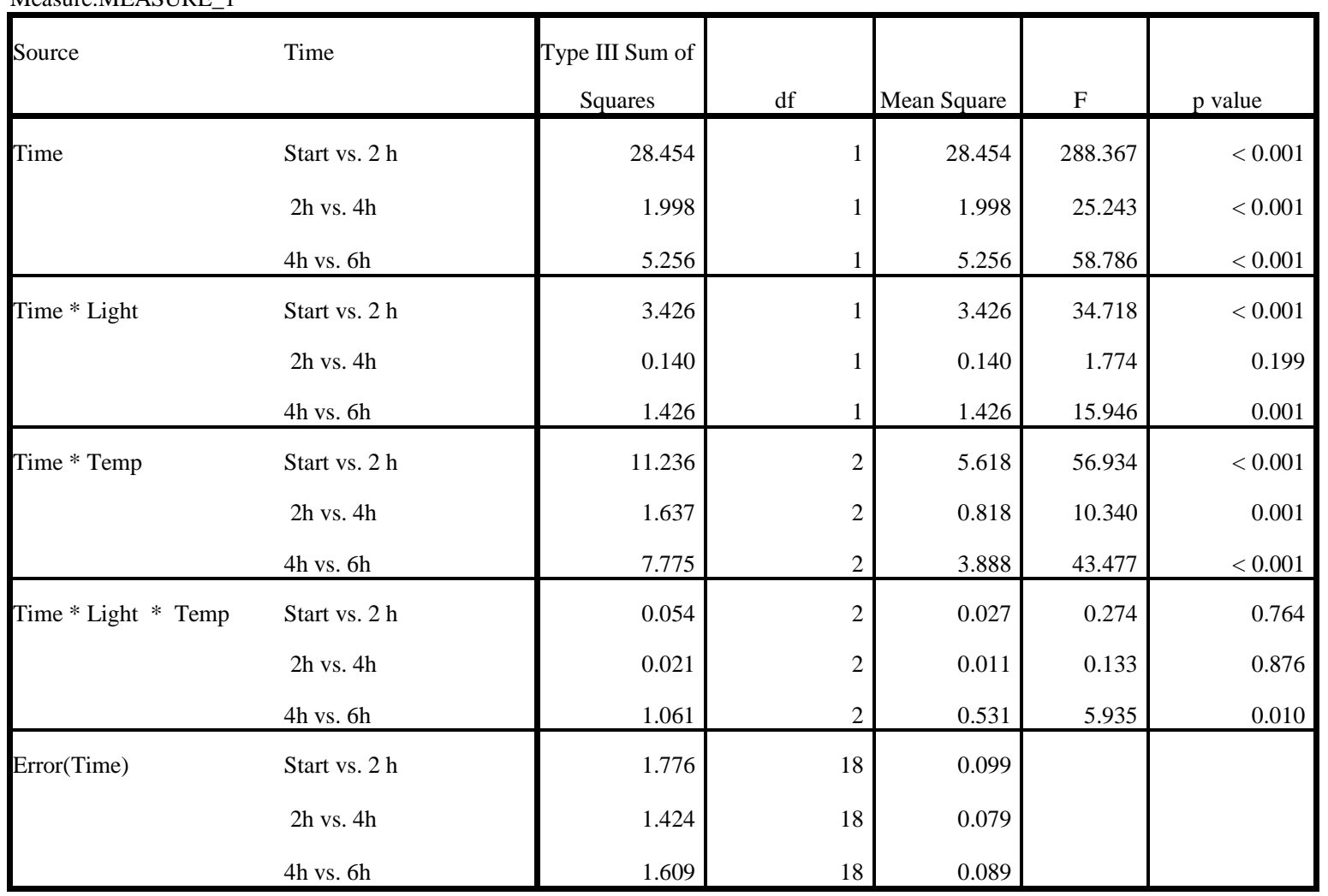


Table A6.5.3: Post hoc tests using Tukey HSD, as assumptions of sphericity were assumed, comparing the difference in mean $(n=4 \pm S E)$ of square root transformed $\mathrm{rETR}_{\max }$ in each treatment between the time-points in a rmANOVA analysis

\begin{tabular}{|c|c|c|c|c|c|}
\hline \multirow{4}{*}{ 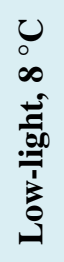 } & & Start & 2 hours & 4 hours & 6 hours \\
\hline & Start & & $<0.001$ & $<0.001$ & $<0.001$ \\
\hline & 2 hours & $<0.001$ & & 0.997 & 0.999 \\
\hline & 4 hours & $<0.001$ & 0.997 & & 1.000 \\
\hline U & & Start & 2 hours & 4 hours & 6 hours \\
\hline$=$ & Start & & $<0.001$ & 0.001 & 0.058 \\
\hline 90 & 2 hours & $<0.001$ & & 0.737 & 0.013 \\
\hline$\stackrel{9}{\Theta}$ & 4 hours & 0.001 & 0.737 & & 0.075 \\
\hline U & & Start & 2 hours & 4 hours & 6 hours \\
\hline m. & Start & & 0.605 & 0.004 & $<0.001$ \\
\hline 90 & 2 hours & 0.605 & & 0.038 & $<0.001$ \\
\hline$\stackrel{9}{\Theta}$ & 4 hours & 0.004 & 0.038 & & $<0.001$ \\
\hline U & & Start & 2 hours & 4 hours & 6 hours \\
\hline$\tilde{E}$ & Start & & $<0.001$ & $<0.001$ & $<0.001$ \\
\hline$\stackrel{I}{I}$ & 2 hours & $<0.001$ & & 0.891 & 0.358 \\
\hline$\overline{\mathbf{I}}$ & 4 hours & $<0.001$ & 0.891 & & 0.749 \\
\hline U & & Start & 2 hours & 4 hours & 6 hours \\
\hline$=$ & Start & & $<0.001$ & $<0.001$ & $<0.001$ \\
\hline$\stackrel{000}{I}$ & 2 hours & $<0.001$ & & 0.073 & 0.511 \\
\hline 五 & 4 hours & $<0.001$ & 0.073 & & 0.566 \\
\hline U & & Start & 2 hours & 4 hours & 6 hours \\
\hline 3 & Start & & 0.032 & 0.632 & 0.001 \\
\hline$\frac{000}{1}$ & 2 hours & 0.032 & & 0.004 & $<0.001$ \\
\hline 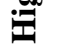 & 4 hours & 0.632 & 0.004 & & 0.01 \\
\hline
\end{tabular}


Table A6.5.4: Post hoc tests using Tukey HSD, as assumptions of sphericity were assumed, comparing the difference in mean $\left(n=4 \pm \mathrm{SE}\right.$ ) of square root transformed $\mathrm{rETR}_{\max }$ of all treatments at $6 \mathrm{~h}$ using an ANOVA analysis. Significance is assumed when $\mathrm{p}<0.05$.

\begin{tabular}{|c|c|c|c|c|c|c|}
\hline & L8 & L16 & L30 & H8 & H16 & H30 \\
\hline L8 & & $<0.001$ & $<0.001$ & 0.001 & 0.731 & $<0.001$ \\
\hline $\mathbf{L 1 6}$ & $<0.001$ & & $<0.001$ & $<0.001$ & $<0.001$ & $<0.001$ \\
\hline $\mathbf{L 3 0}$ & $<0.001$ & $<0.001$ & & $<0.001$ & $<0.001$ & 0.001 \\
\hline $\mathbf{H 8}$ & 0.001 & 0.014 & $<0.001$ & & $<0.001$ & $<0.001$ \\
\hline $\mathbf{H 1 6}$ & 0.731 & $<0.001$ & $<.001$ & 0.014 & & $<0.001$ \\
\hline $\mathbf{H 3 0}$ & $<0.001$ & $<0.001$ & 0.001 & $<0.001$ & $<0.001$ & \\
\hline
\end{tabular}

\section{A6.6: Statistical Results for $\mathbf{E}_{\underline{k}}$}

Table A6.6.1: PASW output for mean $(n=4 \pm S E) E_{k}$ using rmANOVA where normality, variance and sphericity had been assumed. Significance is assumed when $\mathrm{p}<0.05$.

Tests of Within-Subjects Effects

Measure:MEASURE_1 Ek

\begin{tabular}{|l|r|r|r|r|r|}
\hline Source Ek & Type III Sum of Squares & df & Mean Square & F & P value \\
\hline Time & 640.907 & 3 & 213.636 & 12.589 & $<0.001$ \\
Time * Light & 347.451 & 3 & 115.817 & 6.825 & 0.001 \\
Time * Temp & 1987.484 & 6 & 331.247 & 19.520 & $<0.001$ \\
Time * Light * Temp & 123.116 & 6 & 20.519 & 1.209 & 0.316 \\
Error(Time) & 916.356 & 54 & 16.970 & & \\
\hline
\end{tabular}


Table A6.6.2: PASW output for mean $(n=4 \pm S E) E_{k}$ tests of between-subjects to find out if the two factors and their interaction had a significant effect on the experiment outcome. Significance is assumed when $\mathrm{p}<0.05$.

Tests of Between-Subjects Effects

Measure:MEASURE_1

\begin{tabular}{|c|c|c|c|c|c|}
\hline Source Ek & $\begin{array}{c}\text { Type III Sum of } \\
\text { Squares }\end{array}$ & $\mathrm{df}$ & Mean Square & $\mathrm{F}$ & $\mathrm{p}$ value \\
\hline Intercept & 34520.291 & 1 & 34520.291 & 3717.684 & $<0.001$ \\
\hline Light & 235.706 & 1 & 235.706 & 25.384 & $<0.001$ \\
\hline Temp & 856.989 & 2 & 428.495 & 46.147 & $<0.001$ \\
\hline Light $*$ Temp & 2.506 & 2 & 1.253 & 0.135 & 0.875 \\
\hline Error & 167.138 & 18 & 9.285 & & \\
\hline
\end{tabular}

Table A6.6.3: PASW output for mean $(n=4 \pm S E) E_{k}$ tests of within-subjects contrasts to find the time-points between which there had been significant effects of factor interactions with time. Significance is assumed when $\mathrm{p}<0.05$.

Tests of Within-Subjects Contrasts

Measure:MEASURE_1

\begin{tabular}{|c|c|c|c|c|c|c|}
\hline Source Ek & Time & $\begin{array}{c}\text { Type III Sum of } \\
\text { Squares } \\
\end{array}$ & df & Mean Square & $\mathrm{F}$ & $\mathrm{p}$ value \\
\hline \multirow[t]{3}{*}{ Time } & Start vs. $2 \mathrm{~h}$ & 254.150 & 1 & 254.150 & 7.561 & 0.013 \\
\hline & $2 \mathrm{~h}$ vs. $4 \mathrm{~h}$ & 306.023 & 1 & 306.023 & 6.776 & 0.018 \\
\hline & $4 \mathrm{~h}$ vs. $6 \mathrm{~h}$ & 332.640 & 1 & 332.640 & 10.000 & 0.005 \\
\hline \multirow[t]{3}{*}{ Time * Light } & Start vs. $2 \mathrm{~h}$ & 429.260 & 1 & 429.260 & 12.770 & 0.002 \\
\hline & $2 \mathrm{~h}$ vs. $4 \mathrm{~h}$ & 13.651 & 1 & 13.651 & 0.302 & 0.589 \\
\hline & $4 \mathrm{~h}$ vs. $6 \mathrm{~h}$ & 66.169 & 1 & 66.169 & 1.989 & 0.175 \\
\hline \multirow[t]{3}{*}{ Time * Temp } & Start vs. $2 \mathrm{~h}$ & 305.541 & 2 & 152.770 & 4.545 & 0.025 \\
\hline & $2 \mathrm{~h}$ vs. $4 \mathrm{~h}$ & 846.645 & 2 & 423.323 & 9.373 & 0.002 \\
\hline & $4 \mathrm{~h}$ vs. $6 \mathrm{~h}$ & 190.563 & 2 & 95.282 & 2.864 & 0.083 \\
\hline \multirow[t]{3}{*}{ Time $*$ Light $*$ Temp } & Start vs. $2 \mathrm{~h}$ & 5.536 & 2 & 2.768 & 0.082 & 0.921 \\
\hline & $2 \mathrm{~h}$ vs. $4 \mathrm{~h}$ & 103.951 & 2 & 51.976 & 1.151 & 0.339 \\
\hline & $4 \mathrm{~h}$ vs. $6 \mathrm{~h}$ & 208.905 & 2 & 104.453 & 3.140 & 0.068 \\
\hline \multirow[t]{3}{*}{ Error(Time) } & Start vs. $2 \mathrm{~h}$ & 605.043 & 18 & 33.613 & & \\
\hline & $2 \mathrm{~h}$ vs. $4 \mathrm{~h}$ & 812.929 & 18 & 45.163 & & \\
\hline & $4 \mathrm{~h}$ vs. $6 \mathrm{~h}$ & 598.781 & 18 & 33.266 & & \\
\hline
\end{tabular}


Table A6.6.4: Post hoc tests using Tukey HSD, as assumptions of sphericity were assumed, comparing the difference in mean $(n=4 \pm S E)$ of $E_{k}$ in each treatment between the time-points in a rmANOVA analysis.

\begin{tabular}{|c|c|c|c|c|c|}
\hline \multirow{4}{*}{ 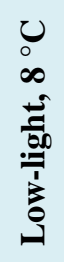 } & & Start & 2 hours & 4 hours & 6 hours \\
\hline & Start & & 0.005 & 0.001 & 0.008 \\
\hline & 2 hours & 0.005 & & 0.901 & 0.99 \\
\hline & 4 hours & 0.001 & 0.901 & & 0.759 \\
\hline ט & & Start & 2 hours & 4 hours & 6 hours \\
\hline$=$ & Start & & 0.078 & 0.088 & 0.699 \\
\hline 90 & 2 hours & 0.078 & & 1.00 & 0.408 \\
\hline$\stackrel{\ominus}{\Theta}$ & 4 hours & 0.088 & 1.000 & & 0.444 \\
\hline U & & Start & 2 hours & 4 hours & 6 hours \\
\hline m. & Start & & 0.962 & 0.19 & 0.005 \\
\hline$\frac{500}{7}$ & 2 hours & 0.962 & & 0.087 & 0.206 \\
\hline ఏ & 4 hours & 0.19 & 0.087 & & 0.206 \\
\hline U & & Start & 2 hours & 4 hours & 6 hours \\
\hline$\stackrel{\Xi}{E}$ & Start & & 0.954 & 0.01 & 0.739 \\
\hline$\stackrel{I}{I}$ & 2 hours & 0.954 & & 0.024 & 0.955 \\
\hline 豆 & 4 hours & 0.01 & 0.024 & & 0.059 \\
\hline ט & & Start & 2 hours & 4 hours & 6 hours \\
\hline$=$ & Start & & 0.976 & 0.6 & 0.999 \\
\hline 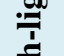 & 2 hours & 0.976 & & 0.377 & 0.993 \\
\hline 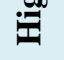 & 4 hours & 0.6 & 0.377 & & 0.52 \\
\hline U & & Start & 2 hours & 4 hours & 6 hours \\
\hline$\stackrel{3}{=}$ & Start & & 0.521 & 0.003 & $<0.001$ \\
\hline$\stackrel{.00}{I}$ & 2 hours & 0.521 & & 0.033 & 0.002 \\
\hline 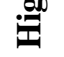 & 4 hours & 0.003 & 0.033 & & 0.326 \\
\hline
\end{tabular}


Table A6.6.5: Post hoc tests using Tukey HSD, as assumptions of sphericity were assumed, comparing the difference in mean $(n=4 \pm S E)$ of $E_{k}$ of all treatments at $6 \mathrm{~h}$ using an ANOVA analysis. Significance is assumed when $\mathrm{p}<0.05$.

\begin{tabular}{|c|c|c|c|c|c|c|}
\hline & L8 & L16 & L30 & H8 & H16 & H30 \\
\hline L8 & & 0.704 & $<0.001$ & 0.282 & 0.163 & $<0.001$ \\
\hline $\mathbf{L 1 6}$ & 0.704 & & $<0.001$ & 0.97 & 0.873 & $<0.001$ \\
\hline $\mathbf{L 3 0}$ & $<0.001$ & $<0.001$ & & 0.001 & 0.002 & 0.180 \\
\hline $\mathbf{H 8}$ & 0.282 & 0.97 & 0.001 & & 0.999 & $<0.001$ \\
\hline $\mathbf{H 1 6}$ & 0.163 & 0.873 & 0.002 & 0.999 & & $<0.001$ \\
\hline $\mathbf{H 3 0}$ & $<0.001$ & $<0.001$ & 0.18 & $<0.001$ & $<0.001$ & \\
\hline
\end{tabular}

\section{A6.7: Statistical Results for NPQ $\underline{\max }$}

Table A6.7.1: PASW output for mean $(n=4 \pm S E) N^{2} Q_{\max }$ using rmANOVA where normality, variance and sphericity had been assumed. Significance is assumed when $\mathrm{p}<0.05$.

\section{Tests of Within-Subjects Effects}

Measure:MEASURE_1

\begin{tabular}{|l|r|r|r|r|r|}
\hline Source & $\begin{array}{c}\text { Type III Sum } \\
\text { of Squares }\end{array}$ & df & Mean Square & F & p value \\
\hline Time & 30.074 & 3 & 10.025 & 143.846 & $<0.001$ \\
Time * Light & 0.292 & 3 & 0.097 & 1.398 & 0.253 \\
Time * Temp & 33.228 & 6 & 5.538 & 79.467 & $<0.001$ \\
Time * Light * Temp & 0.486 & 6 & 0.081 & 1.161 & 0.341 \\
Error(Time) & 3.763 & 54 & 0.070 & & \\
\hline
\end{tabular}


Table A6.7.2: PASW output for mean $(n=4 \pm S E) N Q_{\max }$ tests of between-subjects to find out if the two factors and their interaction had a significant effect on the experiment outcome. Significance is assumed when $\mathrm{p}<0.05$.

\section{Tests of Between-Subjects Effects}

Measure:MEASURE_1

Transformed Variable:Average

\begin{tabular}{|l|r|r|r|r|r|}
\hline Source & $\begin{array}{c}\text { Type III Sum of } \\
\text { Squares }\end{array}$ & df & Mean Square & F & p value \\
\hline Intercept & 285.342 & 1 & 285.342 & 8020.169 & $<0.001$ \\
Light & 0.036 & 1 & 0.036 & 1.007 & 0.329 \\
Temp & 24.386 & 2 & 12.193 & 342.710 & $<0.001$ \\
Light * Temp & 0.234 & 2 & 0.117 & 3.288 & 0.061 \\
Error & 0.640 & 18 & 0.036 & & \\
\hline
\end{tabular}

Table A6.7.3: PASW output for mean $(n=4 \pm S E) N^{2} Q_{\max }$ tests of within-subjects contrasts to find the timepoints between which there had been significant effects of factor interactions with time. Significance is assumed when $\mathrm{p}<0.05$.

Tests of Within-Subjects Contrasts

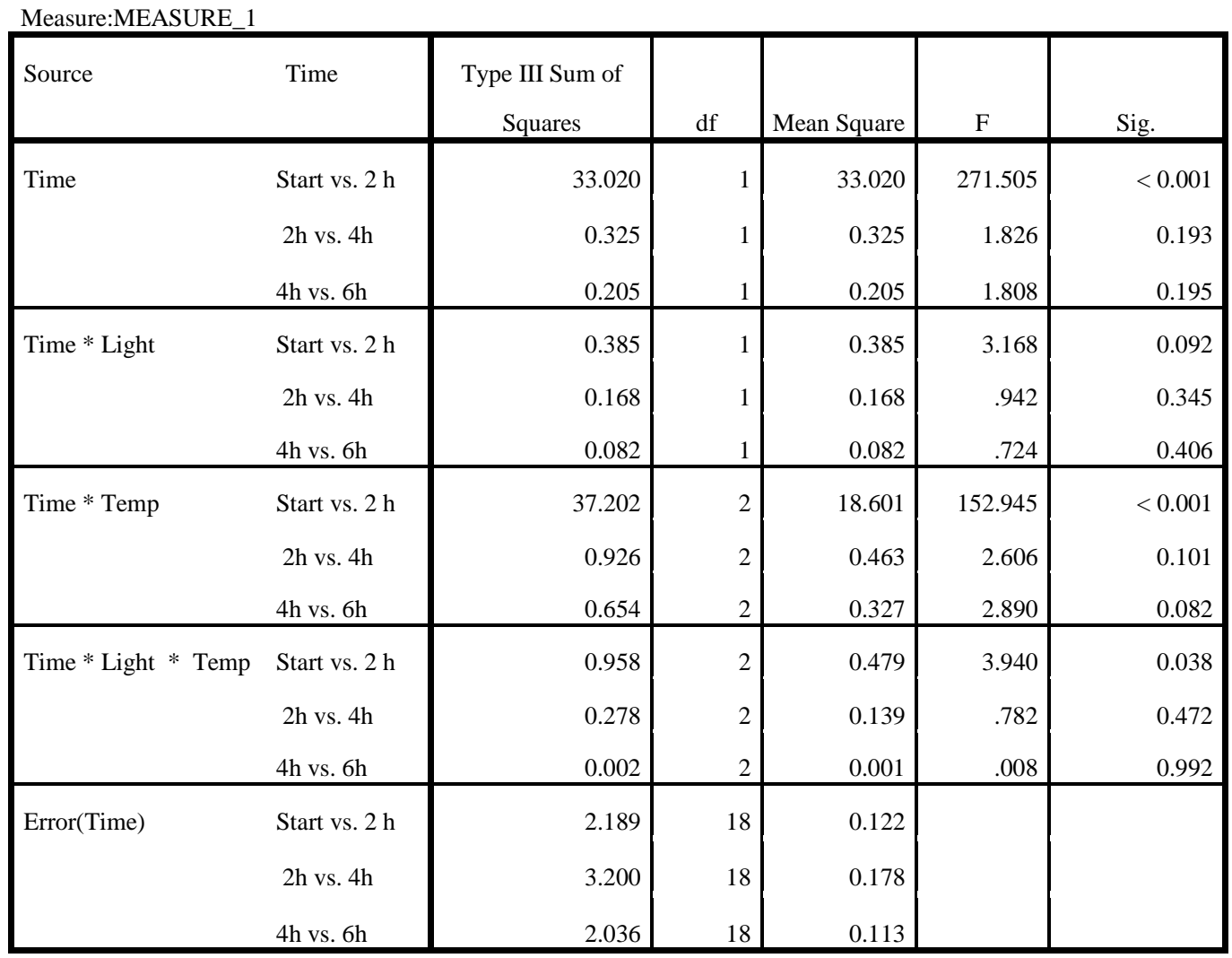


Table A6.7.4: Post hoc tests using Tukey HSD, as assumptions of sphericity were assumed, comparing the difference in mean $(n=4 \pm S E)$ of $\mathrm{NPQ}_{\max }$ in each treatment between the time-points in a rmANOVA analysis. Significance is assumed when $\mathrm{p}<0.05$.

\begin{tabular}{|c|c|c|c|c|c|}
\hline \multirow{4}{*}{ 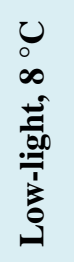 } & & Start & 2 hours & 4 hours & 6 hours \\
\hline & Start & & $<0.001$ & $<0.001$ & $<0.001$ \\
\hline & 2 hours & $<0.001$ & & 0.207 & 0.115 \\
\hline & 4 hours & $<0.001$ & 0.207 & & 0.982 \\
\hline \multirow{4}{*}{ 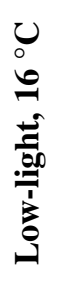 } & & Start & 2 hours & 4 hours & 6 hours \\
\hline & Start & & 0.238 & 0.045 & 0.225 \\
\hline & 2 hours & 0.238 & & 0.743 & 1.000 \\
\hline & 4 hours & 0.045 & 0.743 & & 0.764 \\
\hline \multirow{4}{*}{ 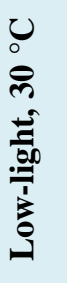 } & & Start & 2 hours & 4 hours & 6 hours \\
\hline & Start & & 0.212 & 0.883 & 0.465 \\
\hline & 2 hours & 0.212 & & 0.547 & 0.935 \\
\hline & 4 hours & 0.883 & 0.547 & & 0.865 \\
\hline \multirow{4}{*}{ 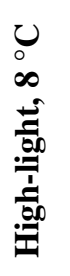 } & & Start & 2 hours & 4 hours & 6 hours \\
\hline & Start & & $<0.001$ & $<0.001$ & $<0.001$ \\
\hline & 2 hours & $<0.001$ & & 0.064 & 0.005 \\
\hline & 4 hours & $<0.001$ & 0.064 & & 0.502 \\
\hline \multirow{4}{*}{ 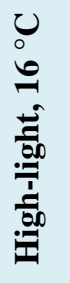 } & & Start & 2 hours & 4 hours & 6 hours \\
\hline & Start & & 0.180 & 0.025 & 0.042 \\
\hline & 2 hours & 0.180 & & 0.659 & 0.816 \\
\hline & 4 hours & 0.025 & 0.659 & & 0.991 \\
\hline \multirow{4}{*}{ 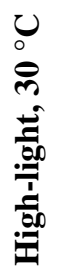 } & & Start & 2 hours & 4 hours & 6 hours \\
\hline & Start & & 0.885 & 0.972 & 0.856 \\
\hline & 2 hours & 0.885 & & 0.991 & 0.458 \\
\hline & 4 hours & 0.972 & 0.991 & & 0.624 \\
\hline
\end{tabular}


Table A6.7.5: Post hoc tests using Tukey HSD, as assumptions of sphericity were assumed, comparing the difference in mean $(n=4 \pm S E)$ of $N^{2} Q_{\max }$ at 6 hours between all the treatments using an ANOVA analysis. Significance is assumed when $\mathrm{p}<0.05$.

\begin{tabular}{|c|c|c|c|c|c|c|}
\hline & L8 & L16 & L30 & H8 & H16 & H30 \\
\hline L8 & & $<0.001$ & $<0.001$ & 0.958 & $<0.001$ & $<0.001$ \\
\hline L16 & $<0001$ & & 1.000 & $<0.001$ & 0.996 & 0.981 \\
\hline L30 & $<0.001$ & 1.000 & & $<0.001$ & 1.000 & 0.924 \\
\hline H8 & 0.958 & $<0.001$ & $<0.001$ & & $<0.001$ & $<0.001$ \\
\hline H16 & $<0.001$ & 0.996 & 1.000 & $<0.001$ & & 0.843 \\
\hline H30 & $<0.001$ & 0.981 & 0.924 & $<0.001$ & 0.843 & \\
\hline
\end{tabular}




\section{References}

Abrego, D., K. E. Ulstrup, B. L. Willis, and M. J. H. Van Oppen. 2008. Species-specific interactions between algal endosymbionts and coral hosts define their bleaching response to heat and light stress. Proceedings of The Royal Society Biological Sciences 275:2273-2282.

Apprill, A. M. and R. D. Gates. 2007. Recognizing diversity in coral symbiotic dinoflagellate communities. Molecular Ecology 16:1127-1134.

Arora, A., R. K. Sairam, and G. C. Srivastava. 2002. Oxidative stress and antioxidative system in plants. Current Science 82:1227-1236.

Baeza, J. A. 2007. The origins of symbiosis as a lifestyle in marine crabs (genus Petrolisthes) from the eastern Pacific: Does interspecific competition play a role? Revista de Biologia Marina y Oceanografia 42:7-21.

Baghdasarian, G. and L. Muscatine. 2000. Preferential Expulsion of Dividing Algal Cells as a Mechanism for Regulating Algal-Cnidarian Symbiosis. Biological Bulletin 199:278-286.

Baillie, B. K., C. A. Belda-Baillie, V. Silvestre, M. Sison, A. V. Gomez, E. D. Gomez, and V. Monje. 2000. Genetic variation in Symbiodinium isolates from giant clams based on randomamplified-polymorphic DNA (RAPD) patterns. Marine Biology 136.

Baker, A. C. 2001. Reef corals bleach to survive change. Nature 411:765-766.

Baker, A. C. 2003. Flexibility and Specificity in Coral-Algal Symbiosis: Diversity, Ecology and Biogeography of Symbiodinium. Annual Review of Ecology, Evolution and Systematics 34:661-689.

Baker, A. C., P. W. Glynn, and B. Riegl. 2008. Climate change and coral reef bleaching: An ecological assessment of lontg-term impacts, recovery trends and future outlook. Estuarine Coastal and Shelf Science 80:435-471.

Banaszak, A. T., T. C. LaJeunesse, and R. K. Trench. 2000. The synthesis of mycosporine-like amina acids (MAAs) by cultured, symbiotic dinoflagellates. Journal of Experimental Marine Biology and Ecology 249:219-233.

Banaszak, A. T., M. G. B. Santos, T. C. LaJeunesse, and M. P. Lesser. 2006. The distribution of mycosporine-like amino acids (MAAs) and the phylogenetic identity of symbiotic dinoflagellates in cnidarian hosts from the Mexican Caribbean. Journal of Experimental Marine Biology and Ecology 337:131-146.

Bates, A. 2000. The intertidal distribution of two algal symbionts hosted by Anthopleura xanthogrammica (Brandt 1835). Journal of Experimental Marine Biology and Ecology 249:249-262.

Bergschneider, H. and G. Muller-Parker. 2008. Nutritional role of two algal symbionts in the temperate sea anemone Anthopleura elegantissima Brandt. Biological Bulletin 215:73-88.

Berkelmans, R. and M. J. H. van Oppen. 2006. The role of zooxanthellae in the thermal tolerance of corals: a 'nugget of hope' for coral reefs in an era of climate change. Proceedings of The Royal Society Biological Sciences 273:2305-2312.

Bhagooli, R. and M. Hidaka. 2003. Comparison of stress susceptibility of in hospite and isolated zooxanthellae among five coral species. Journal of Experimental Marine Biology and Ecology 291:181-197.

Bravo, L. A., F. A. Saavedra-Mella, F. Vera, A. Guerra, L. A. Cavieres, A. G. Ivanov, N. P. A. Huner, and L. J. Corcuera. 2007. Effect of cold acclimation on the photosynthetic performance of two ecotypes of Colobanthus quitensis (Kunth) Bartl. Journal of Experimental Botany 58:3581-3590. 
Brown, B. E. 1997. Coral bleaching: causes and consequences. Coral Reefs 16:S129-S138.

Brown, B. E., I. Ambarsari, M. E. Warner, W. K. Fitt, R. P. Dunne, S. W. Gibb, and D. G. Cummings. 1999. Diurnal changes in photochemical efficiency and xanthophyll concentrations in shallow water reef corals: evidence for photoinhibition and photoprotection. Coral Reefs 18:99-105.

Brown, B. E., C. A. Downs, R. P. Dunne, and S. W. Gibb. 2002. Preliminary evidence for tissue retraction as a factor in photoprotection of corals incapable of xanthophyll cycling. Journal of Experimental Marine Biology and Ecology 277:129-144.

Brown, B. E., M. D. A. Tissier, and J. C. Bythell. 1995. Mechanisms of bleaching deduced from histological studies of reef corals sampled during a natural bleaching event. Marine Biology 122:655-663.

Buddemeier, R. W., A. C. Baker, D. G. Fautin, and J. R. Jacobs. 2004. The adaptive hypothesis of bleaching.in E. Rosenberg and Y. Loya, editors. Coral health and disease. Springer-Verlag, Berlin, Germany.

Buddemeier, R. W. and D. G. Fautin. 1993. Coral bleaching as an adaptive mechanism. BioScience 43:320-326.

Buddemeier, R. W. and D. G. Fautin. 1996. Saturation state and the evolution and biogeography of calcification. Bulletin de I'Institut Oceanographique, Monaco Special 14:23-32.

Burton, G. W. 1990. Antioxidants properties of carotenoids. Journal of Nutrition 119:109-111.

Buxton, L., S. Takahashi, R. Hill, and P. J. Ralph. 2012. Variability in the primary site of photosynthetic damage in Symbiodinium sp. (Dinophyceae) exposed to thermal stress. Journal of Phycology 48:117-126.

Bythell, J. C., A. E. Douglas, V. A. Sharp, J. B. Searle, and B. E. Brown. 1997. Algal genotype and photoacclimatory responses of the symbiotic alga Symbiodinium in natural populations of the sea anemone Anemonia viridis. Proceedings: Biological Sciences 264:1277-1282.

Chen, C. A., J.-T. Wang, L.-S. Fang, and Y.-W. Yang. 2005. Fluctuating algal symbiont communities in Acropora palifera (Scleractinia: Acorporidae) from Taiwan. Marine Ecology Progress Series 295:113-121.

Chen, C. A. and Y.-W. Yang. 2005. Symbiont diversity in scleractinian corals from tropical reefs and subtropical non-reef communities in Taiwan. Coral Reefs 24.

Chen, M. C., Y. M. Cheng, P. J. Sung, C. E. Kuo, and L. S. Fang. 2003. Molecular identification of Rab7 (ApRab7) in Aiptasia pulchella and its exclusion from phagosomes harboring zooxanthellae. Biochemical and Biophysical Research Communications 308:586-595.

Cliflo. Retrieved 6 June 2012. http://cliflo.niwa.co.nz. CliFlo: NIWA's National Climate Database on the Web.

Coates, A. G., J. B. Jackson, L. S. Collins, T. M. Cronin, H. J. Dowsett, L. M. Bybell, P. Jung, and J. A. Obando. 1992. Closure of the Isthmus of Panama: The near-shore marine record of Costa Rica and western Panama. Geological Society of America Bulletin 104:814-828.

Coffroth, M. A., D. M. Poland, E. L. Petrou, D. A. Brazeau, and J. C. Holmberg. 2010. Environmental symbiotic acquisition may not be the solution to warming seas for reef-building corals. PLoS One 5(10):e13258.13210.11371/journal.pone.0013258.

Coffroth, M. A. and S. R. Santos. 2005. Genetic Diversity of Symbiotic Dinoflagellated in the Genus Symbiodinium. Protist 156:19-34.

Coleman, A. W. 2003. ITS2 is a double-edged tool for eukaryotic evolutionary comparisons. Trends Genet 19:370-375.

Connell, S. D. and B. M. Gillanders. 2007. Marine Ecology. Oxford University Press. 
Csaszar Jr, O. S., F. Seneca, and M. J. H. van Oppen. 2009. Variation in antioxidant gene expression in the scleratinian coral Acropora millepora under laboratory thermal stress. Marine Ecology Progress Series 392:93-102.

Culver, S. J. 2003. Benthi foraminifera across the Cretaceous-Tertiary (K-T) boundary: a review. Marine Micropaleontology 47:177-226.

Darling, K. F., C. M. Wade, I. A. Stewart, D. Kroon, R. Dingle, and A. J. L. Brown. 2000. Molecular evidence for genetic mixing of Arctic and Antarctic subpolar populations of planktonic foraminifers. Nature 405:43-47.

Davies, P. S. 1991. Effect of daylight variations on the energy budgets of shallow-water corals. Marine Biology 108:137-144.

Davy, S. K., D. Allemand, and V. M. Weis. 2012. Cell Biology of cnidarian-dinoflagellate symbiosis. Microbiology and Molecular Biology Reviews 76:229-261.

Davy, S. K., I. A. N. Lucas, and J. R. Turner. 1996. Carbon budgets in temperate anthozoandinoflagellate symbioses. Marine Biology 126:773-783.

Davy, S. K., I. A. N. Lucas, and J. R. Turner. 1997a. Uptake and Persistence of Homologous and Heterologous Zooxanthellae in the Temperate Sea Anemone Cereus pedunculatus (Pennant). Biological Bulletin 192:208-216.

Davy, S. K. and J. R. Turner. 2003. Early development and acquisition of zooxanthellae in the temperate symbiotic sea anemone Anthopleura balli (Cocks). Biological Bulletin 205:66-72.

Davy, S. K., J. R. Turner, and I. A. N. Lucas. 1997b. The nature of temperate anthozoandinoflagellate symbioses. Proc Eighth Int. Coral Reef Symp. 2:1307-1312.

de Bary, A. 1879. Die Erscheinung der Symbiose. Tubner, Strassbourg.

Diaz-Almeyda, E., P. E. Thome, M. El Hafidi, and R. Iglesias-Prieto. 2011. Differential stability of photosynthetic membranes and fatty acid composition at elevated temperature in Symbiodinium. Coral Reefs 30:217-225.

Dingman, H. C. 1998. Environmental influences on algal symbiont populations in the sea anemone Anthopleura elegantissima. Western Washington University, Washington.

Douglas, A. E. 2003. Coral bleaching-how and why. Marine Pollution Bulletin 46:385-392.

Douglas, A. E. 2008. Conflicts, cheats and the persistence of symbioses. New Phytologists 177:849858.

Douglas, A. E. 2010. The Symbiotic Habit. First edition. Princeton University Press, Princeton, New Jersey.

Dove, S. G., C. Lovell, M. Fine, J. Deckenback, O. Hoegh-Guldberg, R. Iglesias-Prieto, and K. R. N. Anthony. 2008. Host pigments: potential facilitators of photosynthesis in coral symbioses. Plant, Cell and Environment 31:1523-1533.

Downs, C. A., J. E. Fauth, J. C. Halas, P. Dustan, J. Bemiss, and C. M. Woodley. 2002. Oxidative Stress and Seasonal Coral Bleaching. Free Radical Biology \& Medicine 33:533-543.

Drummond, A. J., B. Ashton, S. Buxton, M. Cheung, A. Cooper, C. Duran, M. Field, H. J, M. Kearse, S. Markowitz, R. Moir, S. Stones-Havas, S. Sturrock, T. Thierer, and A. C. C. Wilson. 2011. Geneious v 5.6.2. Available at: http:/www.geneious.com/.

Dubinsky, Z. and I. Berman-Frank. 2001. Uncoupling primary production from population growth in photosynthesizing organisms in aquatic ecosystems. Aquatic Science 63:4-17.

Dunn, S. R., C. E. Schnitzler, and V. M. Weis. 2007. Apoptosis and autophagy as mechanisms of dinoflagellate symbiont release during cnidarian bleaching: every which way you lose. Proceedings of The Royal Society Biological Sciences 274:3079-3085. 
Dunn, S. R., J. C. Thomason, M. D. A. Le Tissier, and J. C. Bythell. 2004. Heat stress induces different froms of cell death in sea anemones and their endosymbiotic algae depending on temperature and duration. Cell Death and Differentiation 11:1213-1222.

Dunn, S. R. and V. M. Weis. 2009. Apoptosis as a post-phagocytic winnowing mechanism in a coraldinoflagellate mutualism. Environmental Microbiology 11:268-276.

Edmunds, P. J. and R. D. Gates. 2003. Has coral bleaching delayed our understanding of fundamental aspects of coral-dinoglagellate symbioses? BioScience 53:976-980.

Falkowski, P. G., Z. Dubinsky, L. Muscatine, and L. R. McCloskey. 1993. Population control in symbiotic corals. BioScience 43:606-611.

Falkowski, P. G. and J. A. Raven. 2007. Aquatic Photosynthesis. 2nd edition. Princeton University Press, Princeton, pp1-484.

Fang, L. S., S. P. Huang, and L. K. L. 1997. High temperature induces the synthesis of heat-shock proteins and the elevation of intracellular calcium in the cora Acropora grandis. Coral Reefs 16:127.

Finney, J. C., D. T. Pettay, E. M. Sampayo, M. E. Warner, H. A. Oxenford, and T. C. LaJeunesse. 2010. The relative significance of host-habitat, depth and geography on the ecology, endemism, and speciation of coral endosymbionts in the genus Symbiodinium. Microbial Ecology 60:250-263.

Fisher, P. L., M. K. Malme, and S. Dove. 2011. The effect of temperature stress on coralSymbiodinium associations containing distinct symbiont types. Coral Reefs?:?

Fitt, W. K., B. E. Brown, M. E. Warner, and R. P. Dunne. 2001a. Coral bleaching: interpretation of thermal tolerance limits and thermal thresholds in tropical corals. Coral Reefs 20:51-65.

Fitt, W. K., B. E. Brown, M. K. Warner, and R. P. Dunne. 2001b. Coral bleaching: interpretation of thermal tolerance limits and thermal thresholds in tropical corals. Coral Reefs 20:51-65.

Fitt, W. K., R. D. Gates, O. Hoegh-Guldberg, J. C. Bythell, J. C. Jatkar, A. G. Grottoli, M. Gomez, P. Fisher, T. C. LaJeuness, O. Pantos, R. Iglesias-Prieto, D. J. Franklin, L. J. Rodrigues, J. M. Torregiani, R. van Woesik, and M. P. Lesser. 2009. Response of two species of Indo-Pacific corals, Porites cylindrica and Stylophora pistillata, to short-term thermal stress: The host does matter in determining the tolerance of corals to bleaching. Journal of Experimental Marine Biology and Ecology 373:102-110.

Fitt, W. K. and R. K. Trench. 1983. Endocytosis of the symbiotic dinoflagellate Symbiodinium microadriaticum freudenthal by endodermal cells of the scyphistomae of Cassiopeia xanmachana and resistance of the algae to host digestion. Journal of Cellular Science 64:195-212.

Focioli, D., P.-L. Merle, C. Caligara, M. Ciosi, C. Muti, P. Francour, C. Cerrano, and D. Allemand. 2011. Symbiont diversity is not involved in depth acclimation in the Mediterranean sea whip Eunicella singularis. Marine Ecology Progress Series 439:57-71.

Frank, A. B. 1877. Uber die biologischen Verhaltnisse des Thallus einiger Krstflechten. Cohn Beitr Biol Pflanz 2:123-200.

Franklin, D. J., C. M. Molina Cedres, and O. Hoegh-Guldberg. 2005. Increased mortality and photoinhibition in the symbiotic dinoflagellates of the Indo-Pacific coral Stylophora pistillata (Esper) after summer bleaching. Marine Biology 149:633-642.

Franklin, E. C., M. Stat, X. Pochon, H. M. Putnam, and R. D. Gates. 2012. GeoSymbio: a hybrid, cloud-based web application of global geospatial bioinformatics and ecoinformatics for Symbiodinium-host symbioses. Molecular Ecology Resources 12:369-373.doi: 310.1111/j.1755-0998.2011.03081.x. 
Freudenthal, H. D. 1962. Symbiodinium gen nov and Symbiodinium microadriaticum sp no, a zooxanthella - taxonomy, life cycle, and morphology. Journal of Protozoology 9:45-52.

Furla, P., D. Allemand, J. M. Shick, C. Ferrier-Pages, S. Richer, A. Plantivaux, P.-L. Merle, and S. Tambutte. 2005. The symbiotic anthozoan: A physiological chimera between alga and animal. Intergrative and Comparitive Biology 45:595-604.

Gascuel, O. 1997. BioNJ: an improved version of the NJ algorithm based on a simple model of sequence data. Mol. Biol. Evol. 14:685-695.

Gast, R. J., D. J. Beaudoin, and D. A. Caron. 2003. Isolation of Symbiotically Expressed Genes from the Dinoflagellate Symbiont of the Solitary Radiolarian Thalassicolla nucleata. Biological Bulletin 204:210-214.

Gates, R. D. and P. J. Edmunds. 1999. The physiological mechanisms of acclimatization in tropical reef corals. American Zoologisy 39:30-43.

Gibbons, C. L. 2008. Carbon Flux in the Temperate Zooxanthellate Sea Anemone Anthopleura aureoradiata. Masters of Science by Thesis. Victoria University, Wellington.

Giuseppe, G. D., F. Erra, F. Dini, C. Alimenti, V. Adriana, B. Pedrini, K. Wuthrich, and P. Luporini. 2011. Antarctic and Arctic populations of the ciliate Euplotes nobilii show common pheromone-mediated cell-cell signalling and cross-mating. PNAS 108:3181-3186.

Gorbunov, M. Y., Z. S. Kolber, and M. P. Lesser. 2001. Photosynthesis and photoprotection in symbiotic corals. Limnology and Oceanography 46:75-85.

Gordon, D., editor. 2009. New Zealand Inventory of Biodiversity. Volume One: Kingdom Animalia. $584 \mathrm{pp}$.

Goss, R., E. A. Pinto, C. Wilhelm, and M. Richter. 2006. The importance of a highly active and $\Delta \mathrm{pH}-$ regulated diatoxanthin epoxidase for the regulation of the PS II antenna function in diadinoxanthin cycle containing algae. Journal of Plant Physiology 163:1008-1021.

Gottschling, M. and J. Plotner. 2004. Secondary structure models of the nuclear internal transcribed spacer regions and 5.8S rRNA in Calciodinelloideae (Peridiniaceae) and other dinoflagellates. Nucleic Acids Research 32:307-315.

Gou, W., J. Sun, X. LI, Y. Zhen, Z. Xin, Z. Yu, and R. Li. 2003. Phylogenetic analysis of a free-living strain of Symbiodinium isolated from Jiaozhou Bay, P.R. China. Journal of Experimental Marine Biology and Ecology 296:135-144.

Gould, S. J. and N. Eldridge. 1977. Punctuated equilibria: the tempo and mode of evolution reconsidered. Paleobiology 3:115-151.

Grant, A. J., D. A. Trautman, S. Frankland, and R. Hinde. 2003. A symbiosome membrane is not required for the actions of two host signalling compounds regulating photosynthesis in symbiotic algae isolated from cnidarians. Comparative Biochemistry and Physiology, Part A 135:337-345.

Guindon, S. and O. Gascuel. 2003. Syst, Biol 52:696-704. hhtp://www.atgc-montpellier.fr/phyml.

Hackett, J. D., D. M. Anderson, D. L. Erdner, and D. Bhattacharya. 2004. Dinoflagellates: A remakable evolutionary experiment. Americal Journal of Botony 91:1523-1534.

Heinz Walz GmbH, editor. 2006. Imaging-PAM M-Series Chlorophyll Fluorometer. 3rd edition. Heinz Walz GmbH, Effeltrich.

Hennige, S. J., D. J. Suggett, M. E. Warner, K. E. McDougall, and D. J. Smith. 2008. Unravelling coral photoacclimation: Symbiodinium strategy and host modification. Proceedings of the 11th International Coral Reef Symposium:Session No 5.

Hennige, S. J., D. J. Suggett, M. E. Warner, K. E. McDougall, and D. J. Smith. 2009. Photobiology of Symbiodinium revisited: bio-physical and bio-optical signatures. Coral Reefs 28:179-195. 
Hill, R., C. M. Brown, K. DeZeeuw, D. A. Campbell, and P. J. Ralph. 2011. Increased rate of D1 in coral symbionts during bleaching is insufficient to counter accelerated photo-inactivation. Limnology and Oceanography 56:139-146.

Hill, R., C. Frankart, and P. J. Ralph. 2005. Impact of bleaching conditions on the components of non-photochemical quenching in the zooxanthellae of a coral. Journal of Experimental Marine Biology and Ecology 322:83-92.

Hill, R., U. Schreiber, R. Gademann, A. W. D. Larkum, M. Kuhl, and P. J. Ralph. 2004. Spatial heterogeneity of photosynthesis and the effect of temperature-induced bleaching conditions in three species of corals. Marine Biology 144:633-640.

Hill, R., K. E. Ulstrup, and P. J. Ralph. 2009. Temperature Induces Changes in Thylakoid Membrane Thermostability of Cultured, Freshly Isolated, and Expelled Zooxanthellar from Scleractinian Corals. Bulletin of Marine Science 85:223-244.

Hirose, M., J. D. Reimer, M. Hidaka, and S. Suda. 2008. Phylogenetic analyses of potentially freeliving Symbiodinium spp. isolated from coral reef sand in Okinawa, Japan. Marine Biology 155:105-112.

Hoegh-Guldberg, O. 1999. Climate change, coral bleaching and the future of the world's coral reefs. Marine and Freshwater Research $\mathbf{5 0 .}$

Hoegh-Guldberg, O. 2004. Coral reefs in a century of rapid environmental change. Symbiosis 37:131.

Hoegh-Guldberg, O. and J. F. Bruno. 2010. The impact of climate change on the world's marine ecosystems. Science 328:1523-1528.

Hoegh-Guldberg, O. and R. J. Jones. 1999. Photoinhibition and photoprotection in symbiotic dinoflagellates from reef-building corals. Marine Ecology Progress Series 183:73-86.

Hoegh-Guldberg, O., P. J. Mumby, A. J. Hooten, R. S. Steneck, P. Greenfield, E. Gomez, C. D. Harvell, P. F. Sale, A. J. Edwards, K. Caldeira, N. Knowlton, C. M. Eakin, R. Iglesias-Prieto, N. Muthiga, R. H. Bradbury, A. Dubi, and M. E. Hatziolos. 2007. Coral Reefs Under Rapid Climate Change and Ocean Acidification. Science 318:1737-1742.

Horton, P. and A. Hague. 1988. Studies on the induction of chlorophyll fluorescence in isolated barley protoplasts. IV. Resolution of non-photochemical quenching. Biochimica et Biophysica Acta 932:107-115.

Howells, E. J., V. H. Betran, N. W. Larsen, L. K. Bay, B. L. Willis, and M. J. H. van Oppen. 2012. Coral thermal tolerance shaped by local adaptation of photosymbionts. Nature Climate Change 2:116-.

Hrbek, T. and A. Meyer. 2003. Closing of the Tethys Sea and the phylogeny of Eurasian killifishes (Cyprinodontiformes: Cyprinodontidae). Journal of Evolutionary Biology 16:17-36.

Huelsenbeck and Ronquist. 2001. Bioinformatics 17:754-755.

Hughes, T. P., A. H. Baird, D. R. Bellwood, M. Card, S. R. Connolly, C. Folke, R. Grosberg, O. HoeghGuldberg, J. B. Jackson, J. Kleypas, J. M. Lough, P. Marshall, M. Nystrom, S. R. Palumbi, S. R. Pandolfi, B. Rosen, and J. Roughgarden. 2003. Climate Change, Human Impacts and the Resilience of Coral Reefs. Science 301:929-933.

Huner, N. P. A., G. Oquist, and F. Sarhan. 1998. Energy balance and acclimation to light and cold. Trends in plant science 3:224-230.

Hunter, C. L., C. W. Morden, and C. M. Smith. 1997. The utility of ITS sequences in assessing relationships among zooxanthellae and coral. Proceedings of the 8th International Coral Reef Symposium 2:1599-1602. 
Hunter, R. L., T. C. LaJeuness, and S. R. Santos. 2007. Structure and Evolution of the rDNA Internal Transcribed Spacer (ITS) Region 2 in the Symbiotic Dinoflagellates (Symbiodinium, Dinophyta). Journal of Phycology 43:120-128.

Iglesias-Prieto, R. 2005. The photosynthetic apparatus of Symbiodinium.in World Bank/GEF Targeted Research Group on Coral Bleaching and Related Ecological Factors (BWG), Puerto Morelos, Mexico.

Iglesias-Prieto, R. and R. K. Trench. 1994. Acclimation and adaptation to irradiance in symbiotic dinoflagellates. I. Responses of the photosynthetic unit to changes in photon flux density. Marine Ecology Progress Series 113:163-175.

Jeffrey, S. W. and F. T. Haxo. 1968. Photosynthetic pigments of symbiotic dinoflagellates (Zooxanthellae) from corals and clams. Biological Bulletin 135:149-165.

Jeong, H. J., Y. D. Yoo, N. S. Kang, A. S. Lim, K. A. Seong, S. Y. Lee, M. J. Lee, K. H. Lee, H. S. Kim, W. Shin, S. W. Nam, W. Yih, and K. Lee. 2012. Heterotrophic feeding as a newly identified survival strategy of the dinoflagellate Symbiodinium. PNAS Early Edition.

Jones, R. J. 2004. Testing the 'photoinhibition' model of coral bleaching using chemical inhibitors. Marine Ecology Progress Series 284:133-145.

Jones, R. J., O. Hoegh-Guldberg, A. W. D. Larkum, and U. Schreiber. 1998. Temperature-induced bleaching of corals begins with impairment of the $\mathrm{CO}_{2}$ fixation mechanism in zooxanthellae. Plant, Cell and Environment 21:1219-1230.

Jones, R. J. and A. P. Kerswell. 2003. Phytotoxicity of Photosystem II (PSII) herbicides to coral. Marine Ecology Progress Series 261:149-159.

Jones, R. J., S. Ward, A. Y. Amri, and O. Hoegh-Guldberg. 2000. Changes in quantum efficiency of Photosystem II of symbiotic dinoflagellates of corals after heat stress, and of bleached corals sampled after the 1998 Great Barrier Reef mass bleaching event. Marine and Freshwater Research 51:63-71.

Karako-Lambert, S., D. J. Katcoff, Y. Achituv, Z. Dubinsky, and N. Stambler. 2004. Do clades of symbiotic dinoflagellates in scleractinian corals of the Gulf of Eilat (Red Sea) differ from those of other coral reefs? Journal of Experimental Marine Biology and Ecology 311:301314.

Kazandjian, A., V. A. Shepherd, M. Rodriguez-Lanetty, W. Nordemeier, A. W. D. Larkum, and R. G. Quinnell. 2008. Isolation of symbiosomes and the symbiosome membrane complex from the zoanthid Zoanthus robustus. Phycologia 47:294-306.

Kemp, D. W., C. A. Oakley, D. J. Thornhill, L. A. Newcomb, G. W. Schmidt, and W. K. Fitt. 2011. Catastrophic mortality on inshore coral reefs of the Florida Keys due to severe lowtemperature stress. Global Change Biology 17:3468-3477.

Kern, J. and A. Guskov. 2011. Lipids in photosystem II: Multifunctional cofactors. Journal of Photochemistry and Photobiology B: Biology 104:19-34.

Kerrigan, A. M. and G. D. Brown. 2009. C-type lectins and phagocytosis. Immunobiology.

Kinzie, R. A. I., M. Takayama, S. R. Santos, and M. A. Coffroth. 2001. The adaptive bleaching hypothesis: Experimental tests of critical assumptions. Biological Bulletin 22:51-58.

Kraus, G. H. and E. Weis. 1991. Chlorophyll fluorescence and photosynthesis: The Basics. Annual Review of Plant Physiology and Plant Molecular Biology 42:313-349.

Krause, G. H. 1988. Photoinhibition of photosynthesis. An evaluation of damaging and protective mechanisms. Physiologica Plantarum 74:566-574. 
Kvennefors, E. C. E., W. Leggat, O. Hoegh-Guldberg, B. M. Degnan, and A. C. Barnes. 2008. An ancient and variable mannose-binding lectin from the coral Acropora millepora binds both pathogens and symbionts. Developmental and Comparative Immunology.

LaJeunesse, T. C. 2001. Investigating the biodiversity, ecology and phylogeny of endosymbiotic dinoflagellates in the genus Symbiodiium using the ITS region in search of a "species" level marker. Journal of Phycology 37:866-880.

LaJeunesse, T. C. 2002. Diversity and community structure of symbiotic dinoflagellates from Caribbean coral reefs. Marine Biology 141:387-400.

LaJeunesse, T. C. 2005. Symbiodinium radiations since the Miocene-Pliocene Transition. Molecular Biology and Evolution 22:570-581.

LaJeunesse, T. C., R. Bhagooli, M. Hidaka, L. deVantier, T. Done, G. W. Schmidt, W. K. Fitt, and O. Hoegh-Guldberg. 2004. Closely related Symbiodinium spp. differ in relative dominance in coral reef host communities across environmental, latitudinal and biogeographic gradients. Marine Ecology Progress Series 284:147-161.

LaJeunesse, T. C., W. K. Loh, and R. K. Trench. 2009. Do introduced endosymbiotic dinoflagellates 'take' to new hosts? Biological Invasions 11:995-1003.

LaJeunesse, T. C., W. K. Loh, R. van Woesik, O. Hoegh-Guldberg, G. W. Schmidt, and W. K. Fitt. 2003. Low symbiont diversity in southern Great Barrier Reef corals relative to those of the Caribbean. Limnology and Oceanography 48:2046-2054.

LaJeunesse, T. C., R. Smith, M. Walther, J. Pinzon, D. T. Pettay, M. McGinley, M. Aschaffenburg, P. Medina-Rosas, A. L. Cupul-Magana, L. A. Perez, H. Reyes-Bonilla, and M. E. Warner. 2010. Host-symbiont recombination versus natural selection in the response of coraldinoflagellate symbioses to environmental disturbance. Proceedings of The Royal Society Biological Sciences 277:2925-2934.

LaJeunesse, T. C. and R. K. Trench. 2000. Biogeography of Two Species of Symbiodinium (Freudenthal) Inhabiting the Intertidal Sea Anemone Anthopleura elegantissima (Brandt). Biological Bulletin 199:126-134.

Land Information New Zealand (LINZ). Tides Around New Zealand. New Zealand Government, www.linz.govt.nz/hydro/tidal-info/tidal-intro/nz-tides.

Lesser, M. P. 2006. Oxidative stress in marine enviroments: Biochemistry and physiological ecology. Annual Review of Physiology 68:253-278.

Lesser, M. P. and J. H. Farrell. 2004. Exposure to solar radiation increases damage to both host tissues and algal symbionts of corals during thermal stress. Coral Reefs 23:367-377.

Lewis, C. L. and M. A. Coffroth. 2004. The acquisition of exogenous algal symbionts by an octocoral after bleaching. Science 304:1490-1492.

Lien, Y.-T., Y. Nakano, S. Plathong, H. Fukami, J.-T. Wang, and C. A. Chen. 2007. Occurrence of the putatively heat-tolerant Symbiodinium phylotype $D$ in high-latitudinal outlying coral communities. Coral Reefs 26:35-44.

Lin, K.-L., J.-T. Wang, and L.-S. Fang. 2000. Participation of glycoproteins on zooxanthellal cell walls in the establishment of a symbiotic relatioship with the sea anemone Aiptasia pulchella. Zoological Studies 39:172-178.

Logan, D. D. K., A. C. LaFlemme, V. M. Weis, and S. K. Davy. 2010. Flow-Cytometric Characterization of the Cell-Surface Glycans of Symbiotic Dinoflagellates (Symbiodinium spp.). Journal of Phycology 46:525-533. 
Loh, W. K., T. Loi, D. Carter, and O. Hoegh-Guldberg. 2001. Genetic variability of the symbiotic dinoflagellates from the wide ranging coral species Seriatopora hystrix and Acropora longicyathus in the Indo-West Pacific. Marine Ecology Progress Series 222:97-107.

Loram, J. E., N. Boonham, P. O'Toole, H. G. Trapido-Rosenthal, and A. E. Douglas. 2007. Molecular quantification of symbiotic dinoflagellate algae of the genus Symbiodinium. Biological Bulletin 212:259-268.

Mayfield, A. B. and R. D. Gates. 2007. Osmoregulation in anthozoan-dinoflagellate symbiosis. Cmparative Biochemistry and Physiology, Part A 147:1-10.

McCloskey, L. R. and L. Muscatine. 1994. Production and respiration in the Red Sea coral Stylophora pistillata as a function of depth. Proceedings of The Royal Society Biological Sciences 222:215-230.

McDonald, A. H. H., E. M. Sampayo, T. Ridgway, and M. H. Schleyer. 2008. Latitudinal symbiont zonation in Stylophora pistillata from southeast Africa. Marine Biology 154:209-217.

Meron, D., R. Rodolfo-Metalpa, R. Cunning, A. C. Baker, M. Fine, and E. Banin. 2012. Changes in coral microbial communities in response to a natural pH gradient. International Society for Microbial Ecology Journal 22 March doi:10.1038/ismej.2012.19.

Mieog, J. C., M. J. H. van Oppen, N. E. Cantin, W. T. Stam, and J. L. Olsen. 2007. Real-time PCR reveals a high incidence of Symbiodinium clade $D$ at low levels in four scleractinian corals across the Great Barrier Reef: implications for symbiont shuffling. Coral Reefs 26:449-457.

Miyao, M., M. Ikeuchi, N. Yamamoto, and T.-a. Ono. 1995. Specific Degradation of the D1 Protein of photosystem II by treatment with hydrogen peroxide in darkness: Implications for the mechanism of degradation of the D1 protein under illumination. Biochemistry 34:1001910026.

Montresor, M., C. Lovejoy, L. Orsini, G. Procaccini, and S. Roy. 2003. Bipolar distribution of the cyst-forming dinoflagellate Polarella glacialis. Polar Biology 26:186-194.

Morgan-Kiss, R. M., J. C. Priscu, T. Pocock, L. Gudynaite-Savitch, and N. R. A. Hunter. 2006. Adaptation and Acclimation of Photosynthetic Microorganism to Permanently Cold Environments. Microbiology and Molecular Biology Reviews 70:222-252.

Muller-Parker, G. 1984. Dispersal of zooxanthellae on coral reefs by predators on cnidarians. Biological Bulletin 167:159-167.

Muller-Parker, G. and S. K. Davy. 2001. Temperate and tropical algal-sea anemone symbioses. Invertebrate Biology 120:104-123.

Muller-Parker, G., J. Pierce-Cravens, and B. L. Bingham. 2007. Broad Thermal Tolerance of the Symbiotic Dinoflagellate Symbiodinium muscatinei (Dinophyta) in the Sea Anemone Anthopleura elegantissima (Cnidaria) from Northern Latitudes. Journal of Phycology 43:2531.

Muller, P., X.-P. Li, and K. K. Niyogi. 2001. Non-Photochemical Quenching. A Response to Excess Light Energy. Plant Physiology 125:1558-1566.

Munekage, Y., M. Hashimoto, C. Miyake, K. I. Tomizawa, T. Endo, M. Tasaka, and T. Shikanai. 2004. Cyclic electron flow around photosystem I is essential for photosynthesis. Nature 429:579582.

Muscatine, L. 1967. Glycerol Excretion by Symbiotic Algae from Corals and Tridacna and its Control by the Host. Science 156:516-519.

Muscatine, L., P. G. Falkowski, Z. Dubinsky, P. A. Cook, and L. R. McCloskey. 1989. The effect of external nutrient resources on the population dynamics of zooxanthellae in a reef coral. Proceedings of The Royal Society Biological Sciences 236. 
Muscatine, L., C. Goiran, L. Land, J. Jaubert, J.-P. Cuif, D. Allemand, and D. L. McFadden. 2005. Stable Isotopes (13 C and $15 \mathrm{~N}$ ) of Organic Matrix from Coral Skeleton. Proc Natl Acad Sci USA 102:1525-1530.

Muscatine, L. and I. R. Kaplan. 1994. Resource partitioning by reef corals as determined from stable isotope composition of II $\delta^{15} \mathrm{~N}$ of zooxanthellae and animal tissue versus depth. Pacific Science 48:304-312.

Nataraj, A. J., I. Olivos-Glander, N. Kusukawa, and W. E. J. Highsmith. 1999. Single-strand conformation polymorphism and heteroduplex analysis for gel-based mutation detection. Electrophoresis 20.

Nesa, B. and M. Hidaka. 2009. High zooxanthella density shortens the survival time of coral cell aggregates under thermal stress. Journal of Experimental Marine Biology and Ecology 368:81-87.

Obura, D. O. 2009. Reef corals bleach to resist stress. Marine Pollution Bulletin 58:206-212.

Perez, S. F. 2007. Exploring the cellular mechanisms of Cnidarian bleaching in the sea anemone Aiptasia pallida. Dissertation. Oregon State University, Oregon.

Perez, S. F. and V. M. Weis. 2006. Nitric oxide and cnidarian bleaching: an eviction notice mediates breakdown of a symbiosis. The Journal of Experimental Biology 209:2804-2810.

Phillips, S. K. 2006. Latitudinal diversity of the symbiotic dinoflagellate Symbiodinium in New Zealand. Masters by Thesis. Victoria University, Wellington, New Zealand.

Platt, T., C. L. Gallegos, and H. G. Harrison. 1980. Photoinhibition of photosynthesis in natural assemblages of marine phytoplankton. Journal of Marine Research 38:687-701.

Pochon, X., L. Garcia-Cuetos, A. C. Baker, E. Castella, and J. Pawlowski. 2007. One-year survey of a single Micronesian reef reveals extraordinarily rich diversity of Symbiodinium types in soritid foraminifera. Coral Reefs 26:867-882.

Pochon, X. and R. D. Gates. 2010. A new Symbiodinium clade (Dinophyceae) from soritid foraminifera in Hawai'i. Molecular Phylogenetics and Evolution 56:492-497.

Pochon, X., J. I. Montoya-Burgos, B. Stadelmann, and J. Pawlowski. 2006. Moleculal phlyogeny, evolutionary rates, and divergence timing of the symbiotic dinoflagellate genus Symbiodinium. Molecular Phylogenetics and Evolution 38:20-30.

Pochon, X., M. Stat, M. Takabayashi, L. Chasqui, L. J. Chauka, D. D. K. Logan, and R. D. Gates. 2010. Comparison of endosymbiotic and free-living Symbiodinium (Dinophyceae) diversity in Hawaiian reef environment. Journal of Phycology 46:53-65.

Quinn, G. and M. Keough. 2002. Experimental design and data analysis for biologists. Cambridge University Press, Cambridge, United Kingdom.

Ralph, P. J., R. Gademann, A. W. D. Larkum, and M. Kuhl. 2002. Spatial heterogeneity in active chlorophyll fluorescence and PSII activity of coral tissue. Marine Biology 141:639-646.

Ralph, P. J., C. Wilhelm, J. Lavaud, T. Jakob, K. Petrou, and S. A. Kranz. 2010. Fluorescence as a Tool to Understand Changes in Photosynthetic Electron Flow Regulation.in D. J. e. a. Suggett, editor. Chlorophyll a Fluorescence in Aquatic Sciences: Methods and Applications, Developments in Applied Phycology 4. Springer Sciences +Business Media B.V.

Rands, M. L., B. C. Loughman, and A. E. Douglas. 1993. The symbiotic interface in an algainvertebrate symbiosis. Proceedings of The Royal Society Biological Sciences 253:161-165.

Raven, P. H., R. F. Evert, and S. Eichhorn, E. 2005. Biology of Plants - Seventh edition. W H Freeman and Company Publishers, New York. 
Reynolds, J. M., B. U. Bruns, W. K. Fitt, and G. W. Schmidt. 2008. Enhanced photoprotection pathways in symbiotic dinoflagellates of shallow-water corals and other cnidarians. Proc Natl Acad Sci USA 105:13674-13678.

Richier, S., P. Furla, A. Plantivaux, P.-L. Merle, and D. Allemand. 2005. Symbiosis-induced adaptation to oxidative stress. The Journal of Experimental Biology 208:277-285.

Richier, S., C. Sabourault, J. Courtiade, N. Zucchini, D. Allemand, and P. Furla. 2006. Oxidative stress and apoptotic events during thermal stress in the symbiotic sea anemone, Anemonia viridis. FEBS Journal 273:4186-4198.

Robinson, J. D. and M. E. Warner. 2006. Differential impacts of photoacclimation and thermal stress on the photobiology of four different phylotypes of Symbiodinium (Pyrrhophyta). Journal of Phycology 42:568-579.

Rodriguez-Lanetty, M., H. R. Cha, and J. I. Song. 2000. Genetic diversity of symbiotic dinoflagellates associated with anthozoans from Korean waters. Proceedings of the 9th International Coral Reef Symposium, Bali, Indonesia 1:23-27.

Rodriguez-Lanetty, M., S.-J. Chang, and J.-I. Song. 2003. Specificity of two temperate dinoflagellate-anthozoan associations from the north-western Pacific Ocean. Marine Biology 143:1193-1199.

Rodriguez-Lanetty, M., W. K. Loh, D. Carter, and O. Hoegh-Guldberg. 2001. Latitudinal variability in symbiont specificity within the widespread scleractinian coral Plesiastrea versipora. Marine Biology 138:1175-1181.

Rodriguez-Lanetty, M., W. S. Phillips, and V. M. Weis. 2006. Transcriptome analysis of a cnidariandinoflagellate mutualism reveals complex modulation of host gene expression. BMC Genomics 7.

Rowan, R. 1998. Diversity and ecology of zooxanthellae on coral reefs. Journal of Phycology 34:407-417.

Rowan, R. 2004. Thermal adaptation in reef coral symbionts. Nature 430:742.

Rowan, R. and N. Knowlton. 1995. Intraspecific diversity and ecological zonation in coral algal symbiosis. Proc Natl Acad Sci USA 92:3639-3643.

Rowan, R. and D. A. Powers. 1991. A molecular genetic classification of zooxanthellae and the evolution of animal-algal symbioses. Science 251:1348-1351.

Sachs, J. L. and T. P. Wilcox. 2006. A shift to parasitism in the jellyfish symbiont Symbiodinium microadriacticum. Proceedings: Biological Sciences 273:425-429.

Sambrock, J., E. F. Fritsch, and T. Maniatis. 1989. Molecular cloning: a laboratory manual. 2 edition. Cold Spring Harbor Laboratory Press, New York.

Sampayo, E. M., S. Dove, and T. C. LaJeunesse. 2009. Cohesive molecular genetic data delineate species diversity in the dinoflagellate genus Symbiodinium. Molecular Ecology 18:500-519.

Sampayo, E. M., L. Franceschinis, O. Hoegh-Guldberg, and S. Dove. 2007. Niche partitioning of closely related symbiotic dinoflagellates. Molecular Ecology 16:3721-3733.

Sanders, J. G. and S. R. Palumbi. 2011. Populations of Symbiodinium muscatinei show strong biogeographical structuring in the intertidal anemone Anthopleura elegantissima. Biological Bulletin 220:199-208.

Santos, S. R. and M. A. Coffroth. 2003. Molecular genetic evidence that dinoflagellates belonging to the genus Symbiodinium Freudenthal are haploid. Biological Bulletin 204:10-20.

Santos, S. R., D. J. Taylor, R. A. I. Kinzie, M. Hidaka, K. Sakai, and M. A. Coffroth. 2002. Molecular phylogeny of symbiotic dinoflagellates inferred from partial chloroplast large subunit (23S)-rDNA sequences. Molecular Phylogenetics and Evolution 23:97-111. 
Savage, A. M., M. S. Goodson, S. Visram, H. Trapido-Rosenthal, J. Wiedenmann, and A. E. Douglas. 2002a. Molecular diversity of symbiotic algae at the latitudinal margins of their distribution: dinoflagellates of the genus Symbiodinium in corals and sea anemones. Marine Ecology Progress Series 244:17-26.

Savage, A. M., H. Trapido-Rosenthal, and A. E. Douglas. 2002b. On the functional significance of molecular variation in Symbiodinium, the symbiotic algae of Cnidaria: photosynthetic response to irradiance. Marine Ecology Progress Series 244:27-37.

Savage, A. M., H. Trapido-Rosenthal, and A. E. Douglas. 2002c. On the functional significance of molecular variation in Symbiodinium, the symbiotic algae of Cnidaria: photosynthetic response to irradiance. Marine Ecology Progress Series 244:27-37.

Sawyer, S. J. and L. Muscatine. 2001. Cellular mechanisms underlying temperature-induced bleaching in the tropical sea anemone Aiptasia pulchella. Journal of Experimental Biology 204:3443-3456.

Saxby, T., W. C. Dennison, and O. Hoegh-Guldberg. 2003. Photosynthetic responses of the coral Montipora digitata to cold temperature stress. Marine Ecology Progress Series 248:85-97.

Schreiber, U. and C. Klughammer. 2008. Non-photochemical fluorescence quenching and quantum yields in PSI and PSII: Analysis of heat-induced limitations using Maxi-Imaging-PAM and Dual-PAM-100. PAM Application Notes 1:15-18.

Schubert, H., M. Andersson, and P. Snoeijs. 2006. Relationship between photosynthesis and nonphotochemical quenching of chlorophyll fluorescence in two red algae with different carotenoid compositions. Marine Biology 149:1003-1013.

Schultz, J., T. Muller, M. Achtziger, P. N. Siebel, T. Dandekar, and M. Wolf. 2006. The internal transcribed spacer 2 database - a web server for (not only) low level phylogenetic analyses. Nucleic Acids Research 34:Web Server Issue doi:10.1093/nar/gk/1129.

Schwarz, J. A., V. M. Weis, and D. C. Potts. 2002. Feeding behaviour and acquistition of zooxanthellae by planula larvae of the sea anemone Anthopleura elegantissima. Marine Biology 140:471-478.

Secord, D. and L. Augustine. 2000. Biogeography and microhabitat variation in temperate algalinvertebrate symbioses: zooxanthellae and zoochlorella in two Pacific intertidal sea anemones, Anthopleura elegantissima and A. xanthogrammica. Invertebrate Biology 119:139-146.

Seibt, C. and D. Schlichter. 2001. Compatible intracellular ion composition of the host improves carbon assimilation by Zooxanthellae in mutualistic symbioses. Naturwissenschaften 88:382-386.

Shick, J. M. and W. C. Dunlap. 2002. Mycosporine-like amino acids and related gadusols: biosynthesis, accumulation, and UV-protective functions in aquatic organisms. Annual Review of Physiology 64:223-262.

Silverstein, R. N., A. M. Correa, and A. C. Baker. 2012. Specificity is rarely absolute in coral-algal symbiosis: implications for cora response to climate change. Proceedings of The Royal Society Biological Sciences doi: 10.1098/rspb.2012.0055.

Smith, D. C. and E. A. Bernays. 1991. Why do so few animals form endosymbiotic associations with photosynthetic microbes? Philosophical Transactions: Biological Sciences 333:225-230.

Smith, D. C. and A. E. Douglas. 1987. The Biology of Symbiosis. Edward Arnold Ltd, London.

Smith, D. J., D. J. Suggett, and N. R. Baker. 2005. Is photoinhibition of zooxanthellae photosynthesis the primary cause of thermal bleaching in corals? Global Change Biology 11:1-11. 
Soltis, P. S., D. E. Sotis, V. Savoloainen, P. R. Crane, and T. G. Barraclough. 2002. Rate heterogeneity among lineages of tracheophytes: intergration of molecular and fossil data and evidence for molecular living fossils. Proc Natl Acad Sci USA 99:4430-4435.

Stanley, G. D. J. 2006. Photosymbiosis and the Evolution of Modern Coral Reefs. Science 312:857858.

Stanley, G. D. J. and D. G. Fautin. 2001. The Origins of Modern Corals. Science 291:1913-1914.

Stanley, G. D. J. and P. K. Swart. 1995. Evolution of the Coral-Zooxanthellae Symbiosis During the Triassice: A Geochemical Approach. Paleobiology 21:179-199.

Stat, M., C. E. Bird, X. Pochon, L. Chasqui, L. J. Chauka, G. T. Concepcion, D. Logan, M. Takabayashi, R. J. Toonen, and R. D. Gates. 2011. Variation in Symbiodinium ITS2 sequence assembleges among coral colonies. PLoS One 6(1):e15854. doi: 15810.11371/journal.pone0015854.

Stat, M., D. Carter, and O. Hoegh-Guldberg. 2006. The evolutionary history of Symbiodinium and scleractinian hosts - Symbiosis, diversity, and the effect of climate change. Perspectives in Plant Ecology, Evolution and Systemics 8:23-43.

Stat, M., W. K. W. Loh, and O. Hoegh-Guldberg. 2008a. Symbiont acquisition strategy drives hostsymbiont associations in the southern Great Barrier Reef. Coral Reefs 27:763-772.

Stat, M., E. Morris, and R. D. Gates. 2008b. Functional diversity in coral-dinoflagellate symbiosis. Proceedings of the National Academy of Sciences 105:9256-9261.

Stat, M., X. Pochon, R. O. M. Cowie, and R. D. Gates. 2009. Specificity in communities of Symbiodinium in corals from Johnston Atoll. Marine Ecology Progress Series 386:83-96.

Steen, R. G. and L. Muscatine. 1984. Daily budgets of photosynthetically fixed carbon in symbiotic zoanthids. Biol Bull mar biol Lab, Woods Hole 167:477-487.

Strychar, K. B., P. W. Sammarco, and T. J. Piva. 2004. Apoptotic and necrotic stages of Symbiodinium (Dinophyceae) cell death activity: bleaching of soft and scleractinian corals. Phycologia 43:768-777.

Stuckey, F. G. 1909. Notes on a New Zealand Actinian, Bunodes aureoradiata. Transactions of the New Zealand Institute41:374-398.

Suggett, D. J. 2008. Photosynthesis and production of hydrogen peroxide by Symbiodinium (Pyrrhophyta) phylotypes with different thermal tolerances. Journal of Phycology 44:1-9.

Sunnucks, P., A. C. C. Wilson, B. Beheregaray, K. Zenger, J. French, and A. C. Taylor. 2000. SSCP is not so difficult: the application and utility of single-stranded conformation polymorphism in evolutionary biology and molecular ecology. Molecular Ecology 9:1699-1710.

Szabo, I., E. Bergantino, and G. M. Giacometti. 2005. Light and oxyenic photosynthesis: energy dissipation as a protection mechanism against photo-oxidation. European Molecular Biology Organization 6:629-634.

Takabayashi, M., L. M. Adams, X. Pochon, and R. D. Gates. 2012. Genetic diversity of free-living Symbiodinium in surface water and sediment of Hawai'i and Florida. Coral Reefs 31:157167.

Takabayashi, M., S. R. Santos, and C. B. Cook. 2004. Mitochondrial DNA phylogeny of the symbiotic dinoflagellates (Symbiodinium, Dinophyta). Journal of Phycology 40:160-164.

Takahashi, S., T. Nakamura, M. Sakamizu, R. V. Woesik, and H. Yamasaki. 2004. Repair machinery of symbiotic photosynthesis as the primary target of heat stress for reef-building corals. Plant Cell Physiology 45:251-255.

Takahashi, S., S. M. Whitney, and M. R. Badger. 2009. Different thermal sensitivity of the repair of photodamaged photosynthetic machinery in cultured Symbiodinium species. Proc Natl Acad Sci USA 106:3237-3242. 
Taylor, D. L. 1974. Symbiotic marine algae: taxonomy and biological fitness. In Vernberg W B (ed) Symbiosis in the Sea. University of South Carolina Press, South Carolina, pp245-262.

Taylor, F. J. R., M. Hoppenrath, and J. F. Saldarriaga. 2008. Dinoflagellate diversity and distribution. Biodiversity and Conservation 17:407-418.

Tchernov, D., M. Y. Gorbunov, C. de Vargas, S. N. Yadav, A. J. Milligan, M. Haggblom, and P. G. Falkowski. 2004. Membrane lipids of symbiotic algae are diagnostic of sensitivity to thermal bleaching in corals. Proc Natl Acad Sci USA 101:13531-13535.

Tchernov, D., H. Kvitt, L. Haramaty, T. S. Bibby, M. Y. Gorbunov, H. Rosenfeld, and P. G. Falkowski. 2011. Apoptosis and the selective survival of host animals following thermal bleaching in zooxanthellate corals. Proceedings of the National Academy of Sciences 108:9905-9909.

Thornhill, D. J., D. W. Kemp, B. U. Bruns, W. K. Fitt, and G. W. Schmidt. 2008. Correspondence between cold tolerance and temperate biogeography in a western atlantic Symbiodinium (Dinophyta) lineaga. Journal of Phycology 44:1126-1135.

Thornhill, D. J., T. C. LaJeunesse, D. W. Kemp, W. K. Fitt, and G. W. Schmidt. 2006. Multi-year, seasonal genotype surveys of coral-algal symbioses reveal prevalent stability or postbleaching reversion. Marine Biology 148:711-722.

Thornhill, D. J., T. C. LaJeunesse, and S. R. Santos. 2007. Measuring rDNA diversity in eukaryotic microbial systems: how intragenomic variation, pseudogenes, and PCR artifacts confound biodiversity estimates. Molecular Ecology 16:5326-5340.

Toller, W. W., R. Rowan, and N. Knowlton. 2001. Zooxanthellae of the Montastraea annularis species complex: Pattern of distribution of four taxa of Symbiodinium on different reefs and across depths. Biological Bulletin 201:348-359.

Trappe, J. M. 2005. A B Frank and mycorrhizae: The challeng to evolutionary and ecologic theory. Mycorrhiza 15:277-281.

Trench, R. K. 1971. The physiology and biochemistry of zooxanthellae symbiotic with marine coelenterates. 1. The assimilation of photosynthetic products of zooxanthellae by two marine coelenterates. Proceedings of The Royal Society Biological Sciences 177:225-235.

Trench, R. K. 1979. The Cell Biology of Plant-Animal Symbiosis. Annual Revies of Plant Physiology 30:485-531.

Ulstrup, K. E. and M. J. H. Van Oppen. 2003. Geographic and habitat partitioning of genetically distinct zooxanthellae (Symbiodinium) in Acropora corals in the Great Barrier Reef. Molecular Ecology 12:3477-3484.

van Oppen, M. J. H. and R. D. Gates. 2006. Conservation genetic and the resilience of reef-building corals. Molecular Ecology 15:3863-3883.

van Oppen, M. J. H., J. C. Mieog, C. A. Sanchez, and K. E. Fabricius. 2005. Diversity of algal endosymbionts (zooxanthellae) in octocorals: the roles of geography and host relationships. Molecular Ecology 14:2403-2417.

Van Oppen, M. J. H., F. P. Palstra, A. M.-T. Piquet, and D. Miller. 2001. Patterns of coraldinoflagellate associations in Acropora: significance of local availability and physiologoy of Symbiodinium strains and host-symbiont selectivity. Proceedings of the Royal Society of London - Biological Sciences 268:1759-1767.

Venn, A. A., J. E. Loram, and A. E. Douglas. 2008a. Photosynthetic Symbioses in Animals. Journal of Experimental Botany 59:1069-1080.

Venn, A. A., J. E. Loram, H. Trapido-Rosenthal, D. A. Joyce, and A. E. Douglas. 2008b. Importance of Time and Place: Patterns in Abundance of Symbiodinium Clades A and B in the Tropical Sea Anemone Condylactis gigantea. Biological Bulletin 215:243-252. 
Venn, A. A., M. A. Wilson, H. G. Trapido-Rosenthal, B. J. Keely, and A. E. Douglas. 2006. The impact of coral bleaching on the pigment profile of the symbiotic alga, Symbiodinium. Plant, Cell and Environment 29:2133-2142.

Verde, E. A. and L. R. McCloskey. 2007. A comparative analysis of the photobiology of zooxanthellae and zoochlorellae symbiotic with the temperate clonal anemone Anthopleura elegantissima (Brandt). III. Seasonal effects of natural light and temperature on photosynthesis and respiration. Marine Biology 152:775-792.

Vidal-Dupiol, J., M. Adjeroud, E. Roger, L. Foure, D. Duval, Y. Mone, C. Ferrier-Pages, E. Tambutte, S. Tambutte, D. Zoccola, D. Allemand, and G. Mitta. 2009. Coral bleaching under thermal stress: putative involvement of host/symbiont recognition mechanisms. BMC Physiology.

Vieira, O. V., R. J. Botelho, and S. Grinstein. 2002. Phagosome maturation: aging gracefully. Biochemistry Journal 366:689-704.

Visram, S., J. Wiedenmann, and A. E. Douglas. 2006. Molecular diversity of symbiotic algae of the genus Symbiodinium (Zooxanthellae) in cnidarians of the Mediterranean Sea. Journal of the Marine Biological Association of the United Kingdom 86:1281-1283.

Voolstra, C. R., J. A. Schwarz, J. Schnetzer, S. Sunagawa, M. K. Desalvo, A. M. Szmant, M. A. Coffroth, and M. Medina. 2009. The host transcriptome remains unaltered during the establishment of coral-algal symbioses. Molecular Ecology 18:1823-1833.

Wakefield, T. S. and S. C. Kempf. 2001. Development of Host- and Symbiont-Specific Monoclonal Antibodies and Confirmation of the Origin of the Symbiosome Membrane in a CnidarianDinoflagellate Symbiosis. Biological Bulletin 200:127-143.

Wang, J.-T. and A. E. Douglas. 1998. Nitrogen recycling or nitrogen conservation in an algainvertebrate symbiosis? The Journal of Experimental Biology 201:2445-2453.

Warner, M. E. and S. Berry-Lowe. 2006. Differential xanthophyll cycling and photochemical activity in symbiotic dinoflagellates in multiple locations of three species of Caribbean coral. Journal of Experimental Marine Biology and Ecology 339:86-95.

Weis, V. M. 2008. Cellular mechanisms of Cnidarian bleaching: stress causes the collapse of symbiosis. The Journal of Experimental Biology 211:3059-3066.

Weis, V. M., S. K. Davy, O. Hoegh-Guldberg, M. Rodriguez-Lanetty, and J. R. Pringle. 2008. Cell Biology in model systems as the key to understanding corals. Trends in Ecology and Evolution 23:369-376.

Wicks, L. C. 2009. Persistence of corals in marginal habitats: the role of the environment, and symbiont diversity and ecophysiology. Thesis. Victoria University, Wellington.

Wicks, L. C., R. Hill, and S. K. Davy. 2010a. The influence of irradiance on tolerance to high and low temperature stress exhibited by Symbiodinium in the coral, Pocillopra damicornis, from the high-latitude reef of Lord Howe Island. Limnology and Oceanography 55:2476-2486.

Wicks, L. C., E. Sampayo, J. P. A. Gardener, and S. K. Davy. 2010b. Local endemicity and high diversity characterise high-latitude coral-Symbiodinium partnerships. Coral Reefs 29:9891003.

Wood-Charleson, E. M., L. L. Hollingworth, D. A. Krupp, and V. M. Weis. 2006. Lectin/glycan interactions play a role in recognition in a coral/dinoflagellate symbiosis. Cellular Microbiology 8:1985-1993.

Wooldridge, S. A. 2009. A new conceptual model for the warm-water breakdown of the coralalgae endosymbiosis. Marine and Freshwater Research 60:483-496. 
Wykoff, D. D., J. P. Davies, A. Melis, and A. R. Grossman. 1998. The Regulation of Photosynthetic Electron Transport during Nutrient Deprivation in Chlamydomonas reinhardtii. Plant Physiology 117:129-139.

Yellowlees, D., T. A. V. Rees, and W. Leggat. 2008. Metabolic interactions between algal symbionts and invertebrate hosts. Plant, Cell and Environment 31:679-694. 